\title{
Analysis of an Inverse Source Problem with Correlation Data in Experimental Aeroacoustics
}

\author{
DisSERTATION \\ zur Erlangung des mathematisch-naturwissenschaftlichen Doktorgrades \\ "Doctor rerum naturalium" \\ im Promotionsprogramm \\ "Mathematical Science" \\ der Georg-August University School of Science (GAUSS)
}

vorgelegt von

Hans-Georg Raumer

aus Stuttgart

Göttingen, 2021 


\section{Betreuungsausschuss}

Prof. Dr. Thorsten Hohage,

Institut für Numerische und Angewandte Mathematik,

Georg-August-Universität Göttingen

PD Dr. habil. Stefan Langer,

Institut für Aerodynamik und Strömungstechnik,

Deutsches Zentrum für Luft- und Raumfahrt (DLR)

Dr. Carsten Spehr,

Institut für Aerodynamik und Strömungstechnik,

Deutsches Zentrum für Luft- und Raumfahrt (DLR)

\section{Mitglieder der Prüfungskommission}

Referent: Prof. Dr. Thorsten Hohage

Koreferent: PD Dr. habil. Stefan Langer

\section{Weitere Mitglieder der Prüfungskommission}

Prof. Dr. Andreas Dillmann,

Institut für Aerodynamik und Strömungstechnik,

Deutsches Zentrum für Luft- und Raumfahrt (DLR)

Prof. Dr. Christoph Lehrenfeld,

Institut für Numerische und Angewandte Mathematik,

Georg-August-Universität Göttingen

Dr. Carsten Spehr

Prof. Dr. Ingo Witt,

Mathematisches Institut,

Georg-August-Universität Göttingen

Tag der mündlichen Prüfung: 20.07.2021 


\section{Abstract}

In aeroacoustic testing one seeks to localize and reconstruct the power of an aeroacoustic source, given correlation estimates of pressure fluctuations in the frequency domain. This inverse problem with operator-valued data is the research object of this thesis.

We establish a framework on infinite-dimensional spaces which allows for a deeper analysis of the problem. In particular, we prove that the power of a spatially uncorrelated source is uniquely determined by the correlation data.

Furthermore, we analyze several reconstruction methods in an infinite-dimensional and discrete setup. In the latter, the concept of weighted norms is introduced together with a theoretical result on on the optimal weighting choice.

Finally, we discuss important aspects regarding the regularization and numerical implementation of source power reconstruction methods. These are illustrated with various numerical experiments. 



\section{Acknowledgements}

I would like to thank everyone who supported the work on this $\mathrm{PhD}$ project - or even made it possible in the first place.

First to mention is my supervisor Prof. Dr. Thorsten Hohage, who took over the supervision of this thesis, although he was already involved in many time-intensive projects. He introduced me into the field of inverse problems and initiated many of the results in this thesis with numerous thoughts, ideas and notes during our discussions. I would like to thank Dr. habil. Stefan Langer for helpful discussions regarding computational aspects of inverse problems and supporting me with his many years of experience as a mathematician at DLR. I also want to thank Dr. Carsten Spehr for his ongoing support in planning and managing this project. Especially for allowing me the freedom to shift the focus of this work to a significantly different subject than initially planned. Thanks to the aeroacoustics working group and the entire department of experimental methods for a friendly and cooperative daily working atmosphere at the DLR in Göttingen. Thanks to Dr. Daniel Ernst for always helping me to connect the mathematical world with the real-world challenges of experimental aeroacoustics.

Morever, I would like to thank Prof. Dr. Roland Griesmaier for his interesting comments on the factorization method that led to the corresponding results in this thesis.

Finally, I want to thank my partner Jannes Heizmann for his emotional support through difficult times and for his talent to get me out of my thoughts with spontaneous excursions and activities. 


\section{Contents}

1. Introduction 9

\begin{tabular}{ll}
\hline 2. Sound Propagation in Homogeneous Flows & $\mathbf{1 5}$
\end{tabular}

2.1. The convected Helmholtz equation . . . . . . . . . . . . . . . . . . . . 15

2.2. The deterministic source problem . . . . . . . . . . . . . . . . . 18

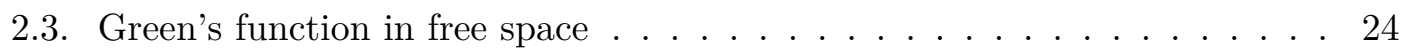

2.4. Green's function in waveguides . . . . . . . . . . . . . . . . . . . . . . . . . 29

2.5. Solution of the deterministic source problem . . . . . . . . . . . . 37

3. Inverse Source Problem in Aeroacoustics 43

3.1. Correlation measurements of random sources . . . . . . . . . . . . . . . . . . 43

3.2. Uniqueness of the inverse source problem . . . . . . . . . . . . . 47

4. Source Power Imaging Methods 53

4.1. Continuous formulations of reconstruction methods . . . . . . . . . . . . 54

4.1.1. Adjoint of the forward operator . . . . . . . . . . . . . . 54

4.1.2. Towards discrete reconstruction techniques . . . . . . . . . . . 56

4.1.3. Covariance matrix fitting (CMF) . . . . . . . . . . . . . . . . 59

4.1.4. Conventional Beamforming . . . . . . . . . . . . . . . . . 59

4.1.5. DAMAS . . . . . . . . . . . . . . . . . . . 61

4.1.6. Tikhonov regularization . . . . . . . . . . . . . . . 6 63

4.2. The factorization method . . . . . . . . . . . . . . 65

4.3. Discrete correlation estimation . . . . . . . . . . . . . . 71

4.3.1. Maximum likelihood estimation of the covariance matrix . . . . . . 71

4.3.2. Discrete noise model . . . . . . . . . . . . . . . 73

\begin{tabular}{ll}
\hline 5. Weighted Data Spaces & 77
\end{tabular}

5.1. Weighted norms . . . . . . . . . . . . . . . . . . 77

$5.2 . \quad$ Weighted Beamforming $\ldots \ldots \ldots \ldots \ldots . \ldots \ldots 78$

5.2.1. Choice of the Gramian matrix . . . . . . . . . . . . . . . 78

5.2 .2 . Variance minimizing weighting . . . . . . . . . . . . 83

5.3. Weighted DAMAS . . . . . . . . . . . . . . . . . . . . . . . . . . . . . 85

5.4. Weighted CMF . . . . . . . . . . . . . . . . . . . . . . 86 
\begin{tabular}{lr}
\hline 6. Computational Aspects & 87
\end{tabular}

6.1. Optimization . . . . . . . . . . . . . . . . 87

6.1.1. Fast Iterative Shrinkage-Thresholding Algorithm . . . . . . . . . . 91

6.1.2. Choice of the regularization parameters . . . . . . . . . . 93

6.2. Discretization . . . . . . . . . . . . . . . . . . . 94

\begin{tabular}{lr}
\hline 7. Numerical Experiments & 97
\end{tabular}

7.1. Synthetic data . . . . . . . . . . . . . . . . . . 97

7.1.1. Influence of additive noise . . . . . . . . . . . . . . . . . . 99

7.1.2. Detection of the support . . . . . . . . . . . . . . . . . . 101

77.1.3. Whitening . . . . . . . . . . . . . . . . . 103

7.1.4. Spatially extended sources . . . . . . . . . . . . . . . . . . . . . 104

7.1.5. The Lepskiì principle. . . . . . . . . . . . . . . . . . . . . . . 107

7.2. Experimental data . . . . . . . . . . . . . . . . . 112

7.2.1. Aspects of the evaluation procedure . . . . . . . . . . . 112

7.2.2. Reconstruction results . . . . . . . . . . . . . . . . . . 113

\begin{tabular}{ll}
\hline 8. Conclusions and Outlook & 115
\end{tabular}

\begin{tabular}{ll}
\hline A. Auxiliary Statements & 117
\end{tabular}

\begin{tabular}{ll}
\hline B. Fundamentals from Calculus and Convex Analysis & 127
\end{tabular}

\begin{tabular}{ll}
\hline Bibliography & 131
\end{tabular} 


\section{1}

\section{Introduction}

\section{Historical background}

The task of localizing or reconstructing sound sources from measurements of the generated sound field arises in many different fields in engineering science. Typical scenarios are for instance

- Analysis of the rail and wheel noise of passing high speed trains (see e.g. [BKP87]),

- speaker localization and speech enhancement for hearing aid devices (see e.g. [GP17, $\mathrm{LCH}^{+} 19$, WTdHEB09]),

$\circ$ analysis of noise sources on wind turbines (see e.g. [OSL07]).

The real-world problem that forms the starting point of this work is the aeroacoustic testing of aircraft (resp. ground vehicle) components. We would like to outline this term a little more precisely.

\section{Aeroacoustics}

Roughly spoken, aeroacustics is concerned with sound that is generated by fluid flows. Either by the turbulent structures within the flow field itself or by fluid-structure interaction. Aeroacoustics as an autonomous field of research has existed since the 1950s initiated by the fundamental work of Sir Michael James Lighthill [Lig52, Lig54]. He derived the famous Lighthill analogy by rearranging the equations of fluid and gas dynamics such that a wave operator occurs on one side of the equation.

\section{Testing of aircraft components}

We would like to emphasize that we are considering experimental methods here i.e. the evaluation of measured acoustic data. Therefore, we will often use the term experimental aeroacoustics to highlight the difference from computational aeroacoustics (CAA). 
Principle of the experimental investigation:

A solid object (for example a scaled model of an aircraft wing) is placed inside an airstream of a wind tunnel. By fluid-structure interactions the object acts as an aeroacoustic sound source. The generated sound field is captured by an acoustic measurement device. From those measurements we seek to quantify and localize the power of the aeroacoustic sound sources.

Measurement device:

The methods discussed in this work are all based on measurements with microphone arrays. Such arrays are also employed in many other fields of application, for example radar $\left[\mathrm{HHL}^{+}\right.$93] or geophysics [BSC01]. Since the 1970s, microphone arrays have also been used in aeroacoustic experiments and measurements (see e.g. [BK76]). Previously, elliptical mirrors were mostly used for this purpose (see e.g. [GJW75]). However, due to their versatility, microphone arrays have become the standard measurement device in most applications. The concept of microphone arrays and elliptic mirrors is illustrated in Figure 1.1 .

\section{Test environment:}

Aeroacoustic measurements are conducted in wind tunnels with open or closed test sections. Each environment has its specific challenges and uncertainties regarding the measurement signals.

In open test sections the microphones are usually placed outside the flow. Thus the sound waves caused by the flow-structure interaction have to pass the turbulent shear layer between core flow and the medium at rest around the microphones. An analytical model that describes the refraction of sound waves at an infinitely thin shear layer was presented by Amiet in 1978 Ami78. Amiet's model was used by Humphreys et al. JBJM98 to include refraction at the shear layer into microphone array methods. An approach to model the coherence loss due to the shear layer by random media theory was presented by Ernst et al. ESB15.

In closed wind tunnels, microphones are typically flush-mounted at the wind tunnel wall. During the experiment, the wind tunnel flow forms a turbulent boundary layer (TBL) that covers the microphones. Therefore, the microphones capture not only the acoustic pressure fluctuations caused by the aeroacoustic effects but also the hydrodynamic ones generated by the TBL.

\section{Correlation-based source imaging}

The measurement environment of aeroacoustic experiments is subject to stochastic effects. Therefore, the raw data (pressure fluctuations in time domain) is usually postprocessed to correlation estimates in frequency domain. We briefly review the most common correlation-based imaging methods. Only a few of them are examined in greater detail in this thesis. 

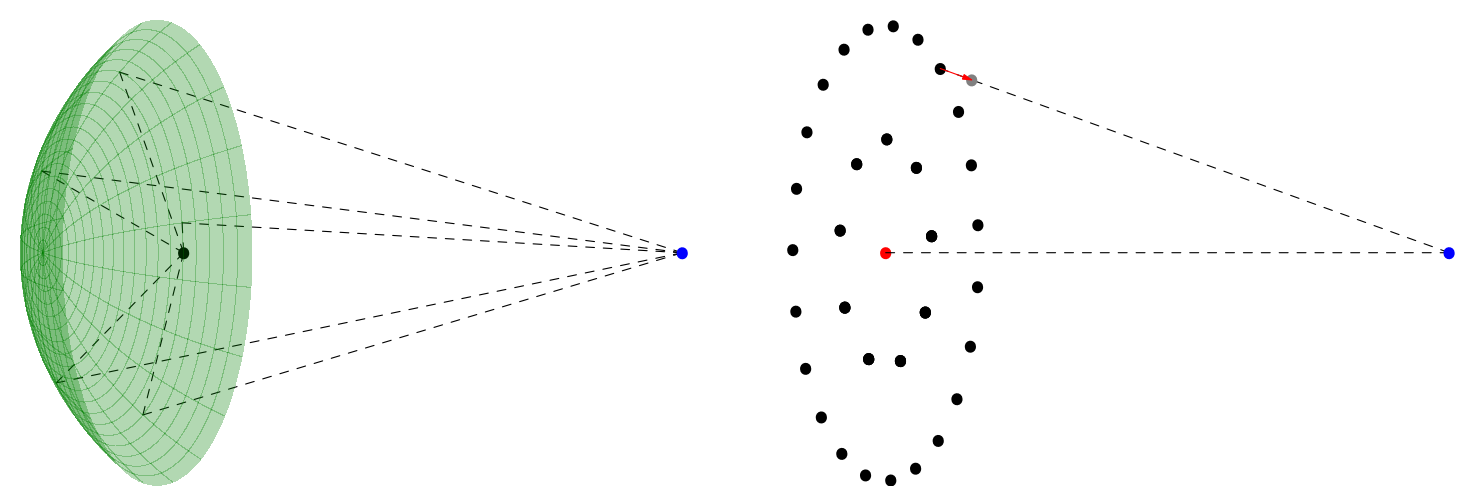

Figure 1.1.: Schematic sketch of the principles of an elliptic mirror (left) and a microphone array (right). The focus point in the source region is indicated in blue and microphones in black. The elliptic mirror aperture contains a single microphone and has to be mechanically adjusted for every focus point. The array consists of many microphones and the adjustments can be done by post-processing of the recorded signals.

\section{Methods that are studied in this thesis:}

\section{Beamforming:}

Beamforming methods rely on the principle of backpropagation. For each location they provide an explicit estimator of the source power and do not require any evaluation of the forward operator. However, they rather capture the main characteristics of the source and do not provide precise reconstructions. This is certainly the reason why Beamforming results are often called dirty map. Beamforming methods are also closely related to helioseismic holography methods that are used to study structures in the interior and far-side of the Sun (see [GFY ${ }^{+} 18$, Lin00, LB00]).

\section{Variational inverse methods:}

Variational inverse methods are based on a matrix (or operator) equation that connects the source power to the observed data, the so-called forward model. A solution is obtained by solving a suitable minimization problem. Such methods either work on the correlation data directly (cross spectral matrix fitting a.k.a. CMF) or on a precomputed Beamforming estimator (deconvolution approach for the mapping of acoustic sources a.k.a. DAMAS).

\section{Methods that are not studied in this thesis:}

\section{CLEAN:}

The CLEAN method has its origin in astronomy and was first introduced by Högbom in 1974 [Hög74]. CLEAN methods work on dirty maps and follow a greedy principle. They iteratively seek to locate the current strongest source, remove its impact on the dirty map and then find the next strongest one. Several variants have been proposed for 
aeroacoustic applications, with CLEAN based on spatial source coherence (CLEAN-SC) Sij07 certainly being the most popular among them.

Eigenvalue-based methods:

Those methods are based on an eigenvalue decomposition of the empirical correlation matrix. The MUSIC algorithm (Multiple Signal Classificatoin) Sch86, WTdHEB09. divides the data into a noise and a signal part, using the magnitude of the eigenvalues. Methods like Generalized Inverse Beamforming Suz08, or Orthogonal Beamforming [SS06] perform source reconstructions on each eigenspace separately.

\section{Bayesian methods:}

In contrast to the frequentist perspective, Bayesian methods (e.g. [Ant12, PAL15]) do not assume the existence of a ground truth but rather treat the source power function as a random variable. The two ingredients of such methods are an initial belief of the source power distribution $\pi_{0}$ (prior) and a probability distribution of the data $\pi_{C \mid q}$, given the source power function (likelihood). The result, the posterior distribution $\pi_{q \mid C}$ of the source power, given the observed data is then provided by the famous Bayes formula

$$
\pi_{q \mid C} \propto \pi_{0} \cdot \pi_{C \mid q}
$$

The crucial steps of most Bayesian approaches are the choice of an appropriate prior distribution and a procedure to obtain point estimators or an representative set of samples from the posterior distribution.

\section{Outline and purpose of this thesis}

We would like to explain what the intention of this work is and point out in what way it seeks to contribute to current research.

\section{Infinite-dimensional modelling:}

When dealing with the aeroacoustic inverse source problem (with correlation data) from a mathematical perspective, one is quickly confronted with the challenge that almost all work on it is based exclusively on discrete formulations. However, to study fundamental properties such as the uniqueness of the problem, an infinite-dimensional formulation is essential. This requirement is the starting point of this work and will be dealt with in Chapter 2 and 3 . Chapter 2 presents the sound propagation model (the convected Helmholtz equation) for several geometrical setups (free-space resp. waveguides in $\mathbb{R}^{2}$ and $\mathbb{R}^{3}$ ). Chapter 3 establishes a mathematical formulation of the inverse problem on function spaces. In the second part we prove one of the main theorems of this work - the uniqueness of the inverse problem. Both chapters will frequently make use of techniques and results that are well-known e.g. in the field of scattering theory (see [CK13]) and adapt them to our framework. 
Connection of mathematical theory with discrete models:

The framework from the first chapters now allows an infinite-dimensional characterization of standard imaging methods (Beamforming, DAMAS and CMF) in Chapter 4. Furthermore those formulations reveal, how the methods are related to each other. In a separate section we discuss the related problem of pure source localization (i.e. the detection of the support of the source power function). It turns out that the principles of the factorization method [KG07] can be carried over to inverse source problem studied here. We then move on towards a discrete setup, which lays the foundation for the investigations in subsequent parts of the thesis. Chapter 5 introduces the concept of finite-dimensional, weighted data spaces. With this setup, many well-known Beamforming variants can be characterized and an optimal weighting with respect to the variance can be derived. Furthermore, this concept can be easily integrated into the CMF resp. DAMAS method.

\section{Numerical treatment:}

The third part of this thesis is concerned with the numerical implementation of the previously discussed methods. As a basic concept, we employ generalized Tikhonov regularization with a quadratic data fidelity term and a penalty functional that consists of a nonnegativity constraint plus $L^{2}-$ and $L^{1}$-norm penalties. In Chapter 6 we discuss several important aspects of the numerical implementation. This includes in particular the choice of a suitable solver for the minimization problem - the fast iterative shrinkagethresholding algorithm (FISTA). Finally, we present several numerical experiments in Chapter 7 to illustrate the ability of the discussed methods. We investigate computations with synthetic data as well as with experimental data.

We conclude this thesis with an outlook on further possible research directions (Chapter 8) and appendices on the proofs of auxiliary statements (Appendix A resp. on basic results from calculus, measure theory and convex analysis (Appendix B).

All in all, this work intends to provide an infinite dimensional mathematical description of the aeroacoustic inverse source problem. The three important topics to be covered are: theory of the problem, methodology and numerics. For the first two, we present new insights and perspectives and prove new theoretical results. The treatment of numerics in this thesis should be seen as a consistent continuation and illustration of the investigations from the first two parts. Moreover, this last part intends to demonstrate a practice-oriented application of inverse methods in experimental aeroacoustics. 



\section{Chapter}

\section{Sound Propagation in Homogeneous Flows}

This chapter treats the basic model of sound propagation which is used in this thesis - the convected Helmholtz equation. We discuss the forward source problem associated with this equation and show existence and uniqueness for a weak formulation of this problem. Furthermore, the fundamental properties of the corresponding Green's functions in free space and waveguide geometries in 2 and 3 spatial dimensions are investigated.

2.1. The convected Helmholtz equation . . . . . . . . . . . . . . . . . . . 15

2.2. The deterministic source problem . . . . . . . . . . . . . . . . 18

2.3. Green's function in free space . . . . . . . . . . . . . . . . . . . . 24

2.4. Green's function in waveguides . . . . . . . . . . . . . . . . . 29

2.5. Solution of the deterministic source problem . . . . . . . . . . . . 37

\subsection{The convected Helmholtz equation}

The forward model in this work considers time harmonic sound propagation within a homogeneous and subsonic flow field. This is of course a simplified modeling of the real measurement conditions since the flow inside a wind tunnel is usually not uniform over the entire domain. Furthermore the geometry of the aeroacoustic model (a scaled aircraft for instance) will be neglected. Despite the simplifications that are introduced, most source reconstruction methods for aeroacoustic experiments in closed test sections are based on those assumptions (see e.g. Sij04, $\mathrm{Ahl16}_{\text {, }} \mathrm{BHE}^{+} 17, \mathrm{MMSS}^{+} 19$ ]).

Let $c$ denote the speed of sound, $\omega$ the angular frequency and $k=\frac{\omega}{c}$ the wavenumber. As discussed above, we consider a constant flow field $\mathbf{u} \in \mathbb{R}^{d}$, where $d \in\{2,3\}$. Without loss of generality we can assume that $\mathbf{u}=\left(u_{1}, 0, \ldots, 0\right)^{\top}$. Moreover, we assume a subsonic regime i.e. the vector $\mathbf{m}=\frac{1}{c} \mathbf{u}$ satisfies $|\mathbf{m}|<1$, where $|\mathbf{m}|$ is usually 
called Mach number. For the time factor convention $e^{-i \omega t}$ and a domain of definition $D \subset \mathbb{R}^{d}$, time-harmonic sound propagation in the flow field $\mathbf{u}$ is described by the convected Helmholtz equation

$$
[k+i \mathbf{m} \cdot \nabla]^{2} p+\Delta p=-Q \quad \text { in } D .
$$

Here $p$ denotes the sound pressure field in frequency domain and $Q$ an acoustic source. Note that in this model $p$ and $Q$ depend only on $\omega$ and $\mathbf{x}$. For $\mathbf{m}=(0, \ldots, 0)^{\top}$, Equation (2.1) reduces to the standard Helmholtz equation that models time harmonic sound propagation in a medium at rest. A very useful relation between the Helmholtz equation with and without convection is provided by the Lorentz transformation (a.k.a. PrandtlGlauert transformation). More precisely, the Lorentz transformation maps solutions of the standard Helmholtz equation to solutions of the convected Helmholtz equation. For the entire thesis we will therefore assume that only the first component of $\mathbf{m}$ may be nonzero i.e.

$$
\mathbf{m}=\left(m_{1}, 0, \ldots, 0\right)^{\top} \quad \text { for } m_{1}=|\mathbf{m}| \in[0,1) .
$$

We further define the scaling factor $\beta \in(0,1]$ by

$$
\beta=\sqrt{1-|\mathbf{m}|^{2}} .
$$

Proposition 2.1 (Lorentz transformation ([HRS20, Proposition 3.1])).

Let $\mathbf{m}=\left(m_{1}, 0, \ldots, 0\right)^{\top} \in \mathbb{R}^{d}$ and $\mathbf{T}=\operatorname{diag}\left(\frac{1}{\beta}, 1, \ldots, 1\right) \in \mathbb{R}^{d \times d}$ with $\beta \in(0,1]$ as in Equation 2.2. We consider an open domain $U \subset \mathbb{R}^{d}$ and functions $\phi_{0} \in C^{2}(U), Q \in$ $C^{0}(U)$ with

$$
\left[\frac{k^{2}}{\beta^{2}}+\Delta\right] \phi_{0}(\mathbf{x})=-Q(\mathbf{x}) \quad \text { on } U
$$

The transformed function

$$
\phi_{\mathbf{m}}(\mathbf{x})=\exp \left(-\frac{|\mathbf{m}| i k}{\beta^{2}} x_{1}\right) \phi_{0}(\mathbf{T x})
$$

then solves the convected Helmholtz equation with a modified source term on a scaled domain. More precisely on $\mathbf{T}^{-1}(U)$ we have

$$
\left[(k+i \mathbf{m} \cdot \nabla)^{2}+\Delta\right] \phi_{\mathbf{m}}(\mathbf{x})=-\exp \left(-\frac{|\mathbf{m}| i k}{\beta^{2}} x_{1}\right) Q(\mathbf{T x}) .
$$

Proof. A more general result for the wave equation in time domain holds with the coor- 
dinate transform

$$
\widehat{\mathbf{T}}\left(\left(\begin{array}{c}
\mathbf{x} \\
t
\end{array}\right)\right):=\left(\begin{array}{c}
\mathbf{T} \mathbf{x} \\
\beta t+\frac{|\mathbf{m}|}{\beta c} x_{1}
\end{array}\right),
$$

see e.g. Cha00, GSAL15. It states that if $\left[\Delta^{2}-\frac{1}{c^{2}} \frac{\partial^{2}}{\partial t^{2}}\right] \psi_{0}(\mathbf{x}, t)=-F(\mathbf{x}, t)$ then the pullback $\psi_{\mathbf{m}}:=\psi_{0} \circ \widehat{\mathbf{T}}$ satisfies

$$
\left[\Delta-\frac{1}{c^{2}}\left(\frac{\partial}{\partial t}+\mathbf{u}_{1} \frac{\partial}{\partial x_{1}}\right)^{2}\right] \psi_{\mathbf{m}}=-F \circ \widehat{\mathbf{T}} .
$$

The statement (2.3) is then obtained from this time domain result for the special case of time harmonic functions $\psi_{0}(\mathbf{x}, t)=\phi_{0}(\mathbf{x}) e^{-i \frac{\omega}{\beta} t}$.

Similarly to the last proposition we have the following reverse result.

Proposition 2.2 (Lorentz transformation - backwards).

Let $\mathbf{m}$ and $\mathbf{T}$ be as in Proposition 2.1. We consider an open domain $U \subset \mathbb{R}^{d}$ and a function $\phi_{\mathbf{m}} \in C^{2}(U), Q \in C^{0}(U)$ with

$$
\left[(k+i \mathbf{m} \cdot \nabla)^{2}+\Delta\right] \phi_{\mathbf{m}}(\mathbf{x})=-Q(\mathbf{x}) \quad \text { on } U .
$$

The transformed function

$$
\phi_{0}(\mathbf{x})=\exp \left(\frac{|\mathbf{m}| i k}{\beta} x_{1}\right) \phi_{\mathbf{m}}\left(\mathbf{T}^{-1} \mathbf{x}\right)
$$

then solves the standard Helmholtz equation at wavenumber $\frac{k}{\beta}$ with a modified source term on a scaled domain. More precisely

$$
\left[\frac{k^{2}}{\beta^{2}}+\Delta\right] \phi_{0}(\mathbf{x})=-\exp \left(\frac{|\mathbf{m}| i k}{\beta} x_{1}\right) Q\left(\mathbf{T}^{-1} \mathbf{x}\right) \quad \text { on } \mathbf{T}(U) .
$$

From now on we will frequently use the differential operator of the convected Helmholtz equation

$$
L_{k}:=k^{2}+2|\mathbf{m}| i k \frac{\partial}{\partial x_{1}}-|\mathbf{m}|^{2} \frac{\partial^{2}}{\partial x_{1}^{2}}+\Delta=(k+i \mathbf{m} \cdot \nabla)^{2}+\Delta
$$

and its transposed operator

$$
L_{k}^{\top}:=k^{2}-2|\mathbf{m}| i k \frac{\partial}{\partial x_{1}}-|\mathbf{m}|^{2} \frac{\partial^{2}}{\partial x_{1}^{2}}+\Delta=(k-i \mathbf{m} \cdot \nabla)^{2}+\Delta .
$$

For the operators defined above we have the following integral formula. 
Proposition 2.3 (Integration by parts formula).

Let $U \subset \mathbb{R}^{d}(d \geq 2)$ be a bounded domain with Lipschitz boundary and $u, v \in C^{2}(\bar{U})$. Then the following integral equation is valid

$$
\begin{aligned}
\int_{U} u(\mathbf{y})\left(L_{k} v\right)(\mathbf{y}) d \mathbf{y}= & \int_{U}\left(L_{k}^{\top} u\right)(\mathbf{y}) v(\mathbf{y}) d \mathbf{y} \\
& +\oint_{\partial U}\{u(\mathbf{y}) \nabla v(\mathbf{y})-v(\mathbf{y}) \nabla u(\mathbf{y})\} \cdot \mathbf{n} d s(\mathbf{y}) \\
& +|\mathbf{m}|^{2} \oint_{\partial U}\left\{v(\mathbf{y}) \frac{\partial u}{\partial y_{1}} n_{1}-u(\mathbf{y}) \frac{\partial v}{\partial y_{1}} n_{1}\right\} d s(\mathbf{y}) \\
& +2 i k|\mathbf{m}| \oint_{\partial U} u(\mathbf{y}) v(\mathbf{y}) n_{1} d s(\mathbf{y})
\end{aligned}
$$

where $\mathbf{n}=\left(n_{1}, \ldots, n_{d}\right)^{\top} \in \mathbb{R}^{d}$ denotes the unit normal vector of $\partial U$, pointing outwards of $U$.

Proof. The proof relies on multidimensional integration by parts and is shifted to Appendix A

\subsection{The deterministic source problem}

Roughly speaking, for a given source function $s$, a (direct) deterministic source problem seeks to solve the partial differential equation

$$
L_{k} u=-s \quad \text { in } D
$$

where $u$ satisfies additional radiation conditions at infinity and boundary conditions at $\partial D$. For this work and especially the analysis of the inverse problem, a weak formulation of the direct problem will be sufficient. These weak formulations will be discussed in this section together with formulations of the radiation conditions for the following two geometrical setups

- $D=\mathbb{R}^{d}$ (free space),

- $D$ is an infinite cylinder (waveguide).

For $U \subset \mathbb{R}^{d}$ we will denote by $W^{k, p}(U)$ the Sobolev space (based on weak derivatives) of order $k$ with exponent $p$ (see e.g. [Alt16]). Furthermore $L_{\text {loc }}^{p}(U)$ denotes the space of functions $f$ on $U$ such that

$$
\int_{K}|f(\mathbf{x})|^{p} d \mathbf{x}<\infty
$$

for every compact set $K \subset U$.

\section{Free space}

We start with the free space problem i.e. $D=\mathbb{R}^{d}$ for $d \in\{2,3\}$ and consider a source function $s \in L^{2}\left(\mathbb{R}^{d}\right)$ with compact support. The source problem will be formulated as a 
variational problem with trial space $L_{\mathrm{loc}}^{2}\left(\mathbb{R}^{d}\right)$ and test space $C_{c}^{\infty}\left(\mathbb{R}^{d}\right)$.

The problem reads: find $u \in L_{\text {loc }}^{2}\left(\mathbb{R}^{d}\right)$ such that for all $\phi \in C_{c}^{\infty}\left(\mathbb{R}^{d}\right)$

$$
\int_{\mathbb{R}^{d}} u L_{k}^{\top} \phi d \mathbf{x}=-\int_{\mathbb{R}^{d}} s \phi d \mathbf{x}
$$

and the back-transformation $b_{u}(\mathbf{x}):=\exp \left(\frac{|\mathbf{m}| i k}{\beta} x_{1}\right) u\left(\mathbf{T}^{-1} \mathbf{x}\right)$ satisfies

$$
\lim _{|\mathbf{x}| \rightarrow \infty}|\mathbf{x}|^{\frac{d-1}{2}}\left(\frac{\partial b_{u}}{\partial|\mathbf{x}|}-i \frac{k}{\beta} b_{u}\right)=0,
$$

uniformly for all directions $\hat{\mathbf{x}}=\frac{\mathbf{x}}{|\mathbf{x}|}$. Here the second condition requires in particular that the derivative $\frac{\partial b_{u}}{\partial|\mathbf{x}|}$ is well defined for $\mathbf{x}$ sufficiently large. This is guaranteed since $s$ has compact support and therefore $L_{k} u=0$ (in the sense of distributions) on $\mathbb{R}^{d} \backslash \operatorname{supp}(s)$. Elliptic regularity results $\left(\left[\right.\right.$ Fol95, Chapter 6]) then yield $u \in C^{\infty}\left(\mathbb{R}^{d} \backslash \operatorname{supp}(s)\right)$. Property (SP1b) is the famous Sommerfeld radiation condition [Som49, p. 192] and characterizes solutions that radiate to infinity. The source Problem $(\overline{\mathrm{SP} 1 \mathrm{a}})+(\mathrm{SP} 1 \mathrm{~b})$ has at most one solution, this fact is transferred from the special case $|\mathbf{m}|=0$.

Theorem 2.4 (Uniqueness of SP1).

If $u_{1}, u_{2} \in L_{\text {loc }}^{2}\left(\mathbb{R}^{d}\right)$ both satisfy SP1a + SP1b , then

$$
u_{1}=u_{2} \text {. }
$$

Proof. We sketch the important steps of the proof. By substracting the variational formulations for $u_{1}$ and $u_{2}$ we conclude, that $u_{1}-u_{2}$ solves (SP1a) + SP1b for $s=0$. By elliptic regularity results $([$ Fol95, Chapter 6$]), u_{1}-u_{2} \in C^{\infty}\left(\mathbb{R}^{d}\right)$. Thus, the backtransformation $b_{u_{1}-u_{2}}$ satisfies

$$
\Delta b_{u_{1}-u_{2}}+\frac{k^{2}}{\beta^{2}} b_{u_{1}-u_{2}}=0
$$

and

$$
\lim _{|\mathbf{x}| \rightarrow \infty}|\mathbf{x}|^{\frac{d-1}{2}}\left(\frac{\partial b_{u_{1}-u_{2}}}{\partial|\mathbf{x}|}-i \frac{k}{\beta} b_{u_{1}-u_{2}}\right)=0
$$

in the classical sense. Hence, $b_{u_{1}-u_{2}}$ must vanish everywhere due to the uniqueness results for the standard Helmholtz equation in $\mathbb{R}^{3}$ [CK13, Proof of Theorem 8.7] and $\mathbb{R}^{2}$ CC06, Special case of Theorem 5.24].

\section{Waveguides}

As an idealized geometry of a windtunnel we consider infinite waveguides with constant cross section area. For the entire thesis we impose the following assumption.

Assumption 2.5 (Shape of cross section).

Let the cross section $\mathcal{A} \subset \mathbb{R}^{d-1}$ with $d \in\{2,3\}$ be a bounded and connected domain 
with Lipschitz boundary i.e. $\partial \mathcal{A}$ can be locally represented as the graph of a Lipschitz continuous function. Furthermore assume that $\mathcal{A}$ satisfies at least one of the following geometric properties

1. $\partial \mathcal{A}$ is of class $C^{2}$,

2. $\mathcal{A}$ is convex.

The waveguide domain is now given by

$$
D=\mathbb{R} \times \mathcal{A}
$$

The walls of the waveguide are assumed to be sound hard, i.e. on the boundary $\partial D$ we impose Neumann boundary conditions in a weak sense. This is achieved by using the corresponding eigenfunctions of the transverse Laplacian

$$
\Delta_{\perp}=\sum_{l=2}^{d} \frac{\partial^{2}}{\partial x_{l}^{2}} .
$$

The eigenfunctions are characterized by the following theorem.

Theorem 2.6 (Spectral Theorem for the Neumann-Laplace Operator).

Let $\mathcal{A} \subset \mathbb{R}^{d-1}$ satisfy Assumption 2.5. Then there exists an orthonormal system of functions $\left\{\psi_{n}\right\}_{n=0}^{\infty} \subset L^{2}(\mathcal{A})$ and real numbers $\left\{\lambda_{n}\right\}_{n=0}^{\infty} \subset[0, \infty)$ such that

(i) $-\Delta \psi_{n}=\lambda_{n} \psi_{n}$,

(ii) $L^{2}(\mathcal{A})=\overline{\operatorname{span}\left\{\psi_{n}\right\}_{n=0}^{\infty}}$,

(iii) $\left\{\psi_{n}\right\}_{n=0}^{\infty} \subset\left(C^{\infty}(\mathcal{A}) \cap W^{2,2}(\mathcal{A})\right)$,

(iv) $\nabla \psi_{n} \cdot \mathbf{n}=0$ on $\partial \mathcal{A}$ for all $n \in \mathbb{N}_{0}$.

Proof. See Appendix A.

Remark 2.7 (Dirichlet boundary conditions).

A similar theorem is valid for the Laplace Operator with homogeneous Dirichlet boundary conditions (see [GT83, Theorem 8.37]). Therefore, the strategies of the proofs in this section are also applicable for sound soft boundaries.

For the further analysis we require additionally that the eigenfunctions $\psi_{n}$ are uniformly bounded and smooth up to the boundary.

Assumption 2.8 (Properties of eigenfunctions).

Let $\mathcal{A} \subset \mathbb{R}^{d-1}$ satisfy Assumption 2.5 and assume further that the $L^{2}$-normalized eigenfunctions $\psi_{n}$ from Theorem 2.6 satisfy

(i) $\sup _{n \in \mathbb{N}_{0}}\left\|\psi_{n}\right\|_{L^{\infty}(\mathcal{A})}<\infty$,

(ii) $\psi_{n} \in C^{\infty}(\overline{\mathcal{A}})$. 
Note that Assumption (2.8) covers the important case of rectangular waveguides. In that scenario the normalized eigenfunctions are given by

$$
\psi_{n}\left(x_{2}\right)=\sqrt{\frac{2-\delta_{0, n}}{h_{1}}} \cos \left(\frac{n \pi}{h_{1}} x_{2}\right) \quad \text { for } n=0,1,2, \ldots
$$

for $d=2$ and by

$$
\psi_{n, m}\left(x_{2}, x_{3}\right)=\sqrt{\frac{2-\delta_{0, n}}{h_{1}}} \cos \left(\frac{n \pi}{h_{1}} x_{2}\right) \sqrt{\frac{2-\delta_{0, m}}{h_{2}}} \cos \left(\frac{m \pi}{h_{2}} x_{3}\right) \quad \text { for } n, m=0,1,2, \ldots
$$

for $d=3$. Here $\delta_{0, n}$ denotes the Kronecker delta and $h_{1}, h_{2}$ the side lengths of the rectangular cross section.

We define further the cross section coordinates

$$
x_{\perp}:= \begin{cases}\left(x_{2}, x_{3}\right) & \text { for } d=3 \\ x_{2} & \text { for } d=2\end{cases}
$$

in order to introduce the deterministic source problem in waveguides. Again we consider a compactly supported source function $s \in L^{2}(D)$ with $\operatorname{supp}(s) \subset \bar{D}$. The trial space will be $L_{\text {loc }}^{2}(\bar{D})$ and the test space

$$
V^{\mathrm{Neu}}:=\left\{\phi \in C_{c}^{\infty}(\bar{D}): \frac{\partial \phi}{\partial n}=0 \text { on } \partial \mathcal{A}\right\}
$$

Here $\frac{\partial \phi}{\partial n}$ denotes the derivative in normal direction at the boundary. Let $\mathbf{n} \in \mathbb{R}^{d-1}$ denote the outer normal vector of $\partial \mathcal{A}$ then we have the identity

$$
\frac{\partial \phi}{\partial n}\left(x_{1}, x_{\perp}\right)=\nabla_{\perp} \phi\left(x_{1}, x_{\perp}\right) \cdot \mathbf{n},
$$

where $\nabla_{\perp}$ denotes the Gradient operator with respect to the transverse coordinates $x_{\perp}$ i.e.

$$
\nabla_{\perp} \phi\left(x_{1}, x_{\perp}\right)=\left(\frac{\partial \phi\left(x_{1}, x_{\perp}\right)}{\partial x_{2}}, \ldots, \frac{\partial \phi\left(x_{1}, x_{\perp}\right)}{\partial x_{d}}\right)^{\top}
$$

The deterministic source problem in waveguides then reads: find $u \in L_{\text {loc }}^{2}(\bar{D})$ such that for all $\phi \in V^{\mathrm{Neu}}$

$$
\int_{D} u L_{k}^{\top} \phi d \mathbf{x}=-\int_{D} s \phi d \mathbf{x}
$$

Furthermore, the coefficients

$$
b_{u}^{(n)}\left(x_{1}\right):=\int_{\mathcal{A}} b_{u}\left(x_{1}, x_{\perp}\right) \psi_{n}\left(x_{\perp}\right) d x_{\perp}
$$


of the back-transformation $b_{u}(\mathbf{x})=\exp \left(\frac{|\mathbf{m}| i k}{\beta} x_{1}\right) u\left(\mathbf{T}^{-1} \mathbf{x}\right)$ satisfy

$$
\left\{\begin{array}{ll}
\lim _{\left|x_{1}\right| \rightarrow \infty}\left(\frac{\partial}{\partial\left|x_{1}\right|}-i \sqrt{\frac{k^{2}}{\beta^{2}}-\lambda_{n}}\right) b_{u}^{(n)}\left(x_{1}\right)=0 & \text { if } \frac{k^{2}}{\beta^{2}}-\lambda_{n}>0 \\
\limsup _{\left|x_{1}\right| \rightarrow \infty}\left|b_{u}^{(n)}\left(x_{1}\right)\right|<\infty & \text { if } \frac{k^{2}}{\beta^{2}}-\lambda_{n}<0
\end{array} .\right.
$$

Remark 2.9 (Smooth solutions).

Consider a smooth solution $u \in C^{2}(\bar{D})$ of the variational formulation (SP2a) and a continuous source function $s \in C^{0}(\bar{D})$ with compact support. For a fixed test function $\phi \in V^{\mathrm{Neu}}$ we can find a bounded interval $I \subset \mathbb{R}$ such that $\phi$ the support of $\phi$ is contained in the closure of $U=\mathcal{A} \times I$. Note that we can choose I sufficiently large such that $\phi$ vanishes identically in a neighborhood of $\partial U \backslash(\mathbb{R} \times \partial \mathcal{A})$. Moreover $\phi$ satisfies the Neumann boundary condition on all parts of $\partial U$ and the first component of the $d$-dimensional outer normal vector of $\partial U$ is always zero on $\partial U \cap(\mathbb{R} \times \partial \mathcal{A})$. Now we can integrate by parts in (SP2a) (i.e. apply Proposition 2.3). By all the facts mentioned above many boundary expression vanish and the remaining terms read as

$$
\int_{U}\left(L_{k} u\right)(\mathbf{x}) \phi(\mathbf{x}) d \mathbf{x}-\oint_{I \times \partial \mathcal{A}} \phi(\mathbf{x}) \frac{\partial u}{\partial n}(\mathbf{x}) d s(\mathbf{x})=-\int_{U} s(\mathbf{x}) \phi(\mathbf{x}) d \mathbf{x} .
$$

Choosing $\phi \in C_{c}^{\infty}(\Omega)$ we get by the fundamental lemma of calculus of variations (cf. [Alt16, 4.22 p.122]) that

$$
\left(L_{k} u\right)(\mathbf{x})=-s(\mathbf{x}) \text { for } \mathbf{x} \in D
$$

in the strong sense. Hence, from 2.8 we get

$$
\oint_{\partial D} \phi(\mathbf{x}) \frac{\partial u}{\partial n}(\mathbf{x}) d s(\mathbf{x})=0 \text { for all } \phi \in V^{\mathrm{Neu}} .
$$

We sketch how (2.9) implies Neumann boundary conditions in the strong sense for the special case of rectangular waveguides in $\mathbb{R}^{3}$ (the two dimensional case works similar). In that case we have $\mathcal{A}=\left(0, h_{1}\right) \times\left(0, h_{2}\right)$ with $h_{1}, h_{2}>0$. Assume there exists a point $\mathbf{z} \in \partial D$ such that $\frac{\partial u}{\partial n}(\mathbf{z})>0$. As $u \in C^{2}(\bar{D})$ we may assume without loss of generality that $\mathbf{z}=\left(z_{1}, 0, z_{3}\right)$ with $z_{3}<h_{2}$. Moreover, there exists a small $\epsilon>0$ such that $\frac{\partial u}{\partial n}(\mathbf{x})>0$ for $\mathbf{x} \in B_{\epsilon}(\mathbf{z}) \cap \partial D$. Now take a nonnegative function $\varphi \in C_{c}^{\infty}\left(\mathbb{R}^{2}\right)$ that is not the zero function with $\varphi\left(y_{1}, y_{2}\right)=0$ for $\sqrt{y_{1}^{2}+y_{2}^{2}} \geq \epsilon$. Let further $\eta \in C_{c}^{\infty}(\mathbb{R})$ with $0 \leq \eta(t) \leq 1$ for all $t \in \mathbb{R}$ and

$$
\eta(t)= \begin{cases}1 & \text { if } t \in\left(-\frac{h_{1}}{2}, \frac{h_{1}}{2}\right) \\ 0 & \text { if } t \geq \frac{2 h_{1}}{3}\end{cases}
$$

The function $\phi\left(x_{1}, x_{2}, x_{3}\right)=\varphi\left(z_{1}+x_{1}, z_{2}+x_{2}\right) \cdot \eta\left(x_{2}\right)$ now satisfies $\phi \in V^{\mathrm{Neu}}$ and testing with this particular $\phi$ in 2.9 leads to a contradiction to $\frac{\partial u}{\partial n}(\mathbf{x})>0$ for $\mathbf{x} \in B_{\epsilon}(\mathbf{z}) \cap \partial D$. 
Hence, $\frac{\partial u}{\partial n}(\mathbf{x})=0$ for $\mathbf{x} \in \partial D$ in the strong sense.

Vice versa, assume $u \in C^{2}(\bar{D})$ satisfies

$$
\begin{aligned}
& \left(L_{k} u\right)(\mathbf{x})=-s(\mathbf{x}) \text { for } \mathbf{x} \in D \\
& \frac{\partial u}{\partial n}(\mathbf{x})=0 \text { for } \mathbf{x} \in \partial D
\end{aligned}
$$

in the strong sense. Then we can multiply 2.10 by a test function $\phi \in V^{\mathrm{Neu}}$, integrate over $D$ and use integration by parts. Then all boundary integrals vanish and we get that $u$ satisfies the variational formulation (SP2a).

To avoid the resonance case we require that

$$
\frac{k^{2}}{\beta^{2}}-\lambda_{n} \neq 0 \text { for all } n \in \mathbb{N}_{0}
$$

In that case, there exists at most one solution of the deterministic source problem in waveguides.

Theorem 2.10 (Uniqueness of SP2).

Let (2.11) and Assumption 2.8 be satisfied. If $u_{1}, u_{2} \in L_{\text {loc }}^{2}(\bar{D})$ both satisfy (SP2a) + (SP2b), then

$$
u_{1}=u_{2}
$$

Proof. We will again sketch the proof. Similar to the free space problem $u_{1}-u_{2}$ solves (SP2a) + SP2b for $s=0$. Moreover, $u_{1}-u_{2} \in C^{\infty}(D)$ and the back-transformation $b_{u_{1}-u_{2}}$ satisfies

$$
\Delta b_{u_{1}-u_{2}}+\frac{k^{2}}{\beta^{2}} b_{u_{1}-u_{2}}=0
$$

and

$$
\left\{\begin{array}{ll}
\lim _{\left|x_{1}\right| \rightarrow \infty}\left(\frac{\partial}{\partial\left|x_{1}\right|}-i \sqrt{\frac{k^{2}}{\beta^{2}}-\lambda_{n}}\right) b_{u_{1}-u_{2}}^{(n)}\left(x_{1}\right)=0 & \text { if } \frac{k^{2}}{\beta^{2}}-\lambda_{n}>0 \\
\limsup _{\left|x_{1}\right| \rightarrow \infty}\left|b_{u_{1}-u_{2}}^{(n)}\right|<\infty & \text { if } \frac{k^{2}}{\beta^{2}}-\lambda_{n}<0
\end{array} .\right.
$$

We will show that all coefficients $b_{u_{1}-u_{2}}^{(n)}$ vanish. By the Lorentz transformation (i.e. testing (SP2a) with $\phi(\mathbf{T x}) \exp \left(\frac{|\mathbf{m}| i k}{\beta^{2}} x_{1}\right)$, the back-transormation $b_{u_{1}-u_{2}}$ satisfies the variational formulation

$$
\int_{D} b_{u_{1}-u_{2}}\left\{\frac{k^{2}}{\beta^{2}}+\Delta\right\} \phi d \mathbf{x}=0
$$

for all $\phi \in V^{\mathrm{Neu}}$. As the eigenfunctions $\psi_{n}$ are smooth up to the boundary (Assumption 2.8 (ii), we can test in particular with $\phi\left(x_{1}, x_{\perp}\right)=\eta\left(x_{1}\right) \psi_{n}\left(x_{\perp}\right)$ with a function $\eta \in$ 
$C_{c}^{\infty}(\mathbb{R})$. This yields

$$
\begin{aligned}
0 & =\int_{D} b_{u_{1}-u_{2}}\left\{\frac{k^{2}}{\beta^{2}}+\Delta\right\} \phi d \mathbf{x}=\int_{D} b_{u_{1}-u_{2}}\left\{\frac{k^{2}}{\beta^{2}} \eta \psi_{n}+\eta^{\prime \prime} \psi_{n}+\eta \Delta_{\perp} \psi_{n}\right\} d \mathbf{x} \\
& =\int_{\mathbb{R}}\left\{\left(\frac{k^{2}}{\beta^{2}}-\lambda_{n}\right) \eta+\eta^{\prime \prime}\right\} b_{u_{1}-u_{2}}^{(n)} d x_{1} .
\end{aligned}
$$

Equation (2.12) implies that $b_{u_{1}-u_{2}}^{(n)}$ is a distributional solution of a one-dimensional ordinary differential equation and since $b_{u_{1}-u_{2}}^{(n)} \in C^{\infty}(\mathbb{R})$ it is also a strong solution. Thus by standard theory of ordinary differential equations (see e.g. [Tes12, Chapter 3]) we can represent $b_{u_{1}-u_{2}}^{(n)}$ explicitly by

$$
b_{u_{1}-u_{2}}^{(n)}\left(x_{1}\right)=A^{(n)} \exp \left(i \sqrt{\frac{k^{2}}{\beta^{2}}-\lambda_{n} x_{1}}\right)+B^{(n)} \exp \left(-i \sqrt{\frac{k^{2}}{\beta^{2}}-\lambda_{n} x_{1}}\right)
$$

with some constants $A^{(n)}, B^{(n)} \in \mathbb{C}$. By a distinction of cases $\left(\frac{k^{2}}{\beta^{2}}-\lambda_{n}>0\right.$ and $\left.\frac{k^{2}}{\beta^{2}}-\lambda_{n}<0\right)$ we conclude that 2.13 together with the radiation conditions SP2b imply $A^{(n)}=$ $B^{(n)}=0$ for all $n \in \mathbb{N}_{0}$ and hence $b_{u_{1}-u_{2}}^{(n)}=0$.

\subsection{Green's function in free space}

For $\mathbf{y} \in D$ a Green's function $g(\cdot, \mathbf{y})$ of Equation (2.1) is defined as the distributional solution of

$$
[k+i \mathbf{m} \cdot \nabla]^{2} p+\Delta p=-\delta_{\mathbf{y}} \quad \text { in } D .
$$

Here $\delta_{\mathbf{y}}$ denotes the Dirac delta distribution at $\mathbf{y}$. For this section we consider the entire space as domain of definition i.e. $D=\mathbb{R}^{d}$. In that case, $g$ is often called the fundamental solution. Note that Equation (2.14) is not sufficient to determine the Green's function uniquely. However, an additional radiation condition similar as in SP1b allows the selection of physically meaningful solutions.

\section{Standard Helmholtz equation:}

For $\mathbf{m}=0$, Equation (2.1) reduces to the Helmholtz equation. In that case physical meaningful solutions (i.e. outgoing waves) are characterized by the Sommerfeld radiation condition

$$
\lim _{|\mathbf{x}| \rightarrow \infty}|\mathbf{x}|^{\frac{d-1}{2}}\left(\frac{\partial p}{\partial|\mathbf{x}|}-i k p\right)=0, \quad \text { uniformly for all directions } \hat{\mathbf{x}}=\frac{\mathbf{x}}{|\mathbf{x}|} .
$$


The Green's functions satisfying (2.15) are given by [CK13, p. 5 resp. p.74]

$$
\begin{array}{ll}
\text { for } d=3: & g(\mathbf{x}, \mathbf{y})=\frac{e^{i k|\mathbf{x}-\mathbf{y}|}}{4 \pi}, \quad \mathbf{x} \neq \mathbf{y} \\
\text { for } d=2: & g(\mathbf{x}, \mathbf{y})=\frac{i}{4} H_{0}^{(1)}(k|\mathbf{x}-\mathbf{y}|), \quad \mathbf{x} \neq \mathbf{y}
\end{array}
$$

where $H_{0}^{(1)}$ denotes the Hankel function of the first kind of order zero.

\section{Convected Helmholtz equation:}

The solutions presented above can be mapped by means of the Lorentz transformation to the case where $\mathbf{m} \neq 0$. Denote by $g_{0}(\mathbf{x}, \mathbf{y}, k)$ the no-flow Green's function $(\sqrt{2.16},,(2.17))$ at wavenumber $k$. The mapping property of the Lorentz transformation (Proposition 2.1. then implies that the Green's function with convection at wavenumber $k$ is given by

$$
g(\mathbf{x}, \mathbf{y})=\frac{1}{\beta} \exp \left(-\frac{i k}{\beta^{2}}(\mathbf{x}-\mathbf{y}) \cdot \mathbf{m}\right) g_{0}\left(\mathbf{T x}, \mathbf{T y}, \frac{k}{\beta}\right) .
$$

For an explicit representation of $g$ it is convenient to define the Mach norm

$$
|\mathbf{z}|_{\mathbf{m}}=\sqrt{(\mathbf{z} \cdot \mathbf{m})^{2}+\beta^{2}|\mathbf{z}|^{2}},
$$

where $\beta=\sqrt{1-|\mathbf{m}|^{2}}$. With the identity

$$
|\mathbf{T z}|=\frac{1}{\beta}|\mathbf{z}|_{\mathbf{m}}
$$

we obtain explicitly

$$
\begin{aligned}
\text { for } d=3: & g(\mathbf{x}, \mathbf{y})=\frac{\exp \left(\frac{i k}{\beta^{2}}\left(-(\mathbf{x}-\mathbf{y}) \cdot \mathbf{m}+|\mathbf{x}-\mathbf{y}|_{\mathbf{m}}\right)\right)}{4 \pi|\mathbf{x}-\mathbf{y}|_{\mathbf{m}}} \\
\text { for } d=2: & g(\mathbf{x}, \mathbf{y})=\frac{i}{4 \beta} \exp \left(-\frac{i k}{\beta^{2}}(\mathbf{x}-\mathbf{y}) \cdot \mathbf{m}\right) H_{0}^{(1)}\left(\frac{k}{\beta^{2}}|\mathbf{x}-\mathbf{y}|_{\mathbf{m}}\right) .
\end{aligned}
$$

For the remainder of this section, we will study further properties of the free space Green's function.

\section{Asymptotic behavior}

For the standard Helmholtz equation it is well-known that the Green's function behaves asymptotically like an outgoing spherical wave see [CK13, p.22, p.74]. By means of the Lorentz transformation this result can be generalized to the case with subsonic uniform convection. Propagation directions $\hat{\mathbf{x}}$ can be generalized as elements of the unit sphere 

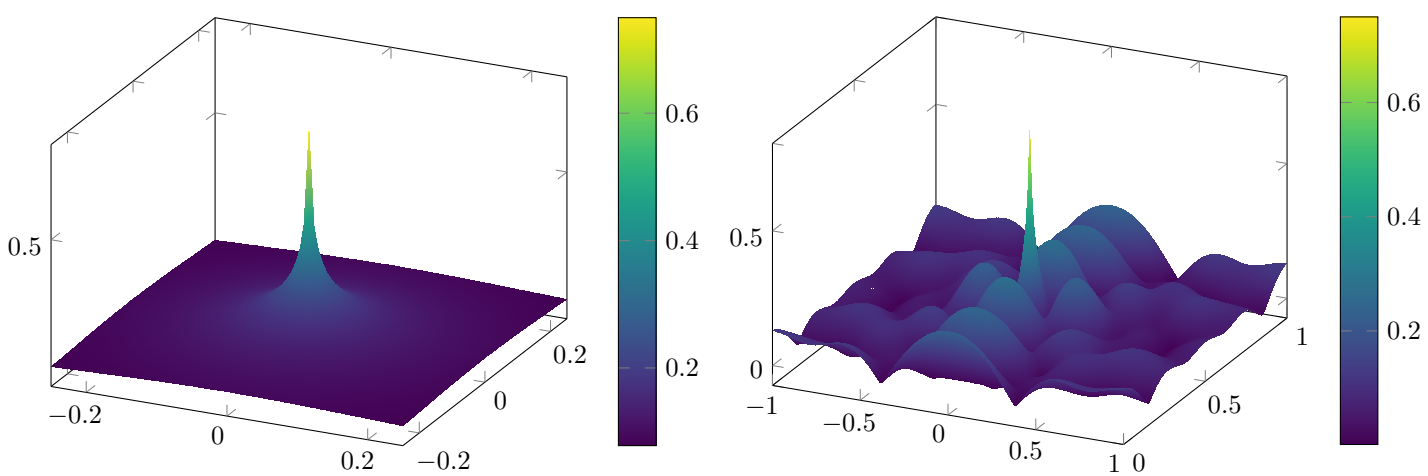

Figure 2.1.: 2D Greens function for $D=\mathbb{R}^{2}$ (left) and $D=\mathbb{R} \times[0,1]$ (right).

Plot of $|g(\cdot, \mathbf{0})|$ for wavenumber $k=18.48$. Values above 0.75 are truncated.

with respect to the Mach norm

$$
\mathbb{S}_{d-1}(\mathbf{m})=\left\{\mathbf{x} \in \mathbb{R}^{d}:|\mathbf{x}|_{\mathbf{m}}=1\right\}
$$

Proposition 2.11 (Asymptotic behavior of Green's function [HRS20, Proposition 3.2]).

Let $B=B_{R}(\mathbf{0})$ be a ball, with radius $R$, then for $\mathbf{x} \in \mathbb{R}^{d} \backslash \bar{B}$ and $\mathbf{y} \in B$ the following asymptotic holds true:

$$
g(\mathbf{x}, \mathbf{y})=C(d) h(\mathbf{x})|\mathbf{x}|^{-\frac{(d-1)}{2}} \exp \left(\frac{i k}{\beta^{2}}(\mathbf{m}-\mathbf{A} \hat{\mathbf{x}}) \cdot \mathbf{y}\right)+\mathcal{O}\left(|\mathbf{x}|^{-\frac{(d+1)}{2}}\right) \quad \text { as }|\mathbf{x}| \rightarrow \infty
$$

with the auxiliary quantities

$$
\begin{array}{llll}
C(2):=\frac{e^{i \frac{\pi}{4}}}{\sqrt{8 \pi k}}, & h(\mathbf{x}):=\exp \left(\frac{i k}{\beta^{2}}\left(|\mathbf{x}|_{\mathbf{m}}-\mathbf{x} \cdot \mathbf{m}\right)\right), & & \\
C(3):=\frac{1}{4 \pi}, & \mathbf{A}:=\mathbf{m m}^{\top}+\beta^{2} \mathbf{I}, & \hat{\mathbf{x}}:=\frac{\mathbf{x}}{|\mathbf{x}|_{\mathbf{m}}} .
\end{array}
$$

The asymptotic formula (2.21) holds uniformly for all $\mathbf{y} \in B$ and all directions $\hat{\mathbf{x}} \in$ $\mathbb{S}_{d-1}(\mathbf{m})$.

Proof. The proof boils down to an application of Proposition 2.1 and the asymptotic behavior of the no-flow Green's function, see [HRS20.

\section{Integrability}

In the subsequent analysis and in future chapters we will need the notion of a HilbertSchmidt operator and Hilbert-Schmidt integral kernels (cf. Proposition and Definition B.2 in the Appendix B). For the uniqueness of the inverse problem, the following two integrability statements will be useful. 
Proposition 2.12 (Hilbert-Schmidt kernel - free field).

For any open, bounded set $B \subset \mathbb{R}^{d}$ the Green's function is square integrable on $B \times B$ i.e.

$$
\int_{B} \int_{B}|g(\mathbf{x}, \mathbf{y})|^{2} d \mathbf{x} d \mathbf{y}<\infty
$$

Moreover, the auxiliary function

$$
h_{B}(\mathbf{y}):=\int_{B}|g(\mathbf{x}, \mathbf{y})|^{2} d \mathbf{x}
$$

belongs to $L^{\infty}(B)$.

Proof. Due to the norm equivalence in finite dimensional spaces and the singular resp. asymptotic behavior of the Bessel functions Wyl76, Chapter 4] [CC06, p. 51], there exist constants $C>0$ such that

$$
|g(\mathbf{x}, \mathbf{y})| \leq C|\mathbf{x}-\mathbf{y}|^{-1}, \quad \mathbf{x} \neq \mathbf{y}
$$

for $d=3$ and

$$
|g(\mathbf{x}, \mathbf{y})| \leq C|\mathbf{x}-\mathbf{y}|^{-1 / 2}, \quad \mathbf{x} \neq \mathbf{y}
$$

for $d=2$. Hence, $g$ is a square integrable integral kernel and therefore defines a HilbertSchmidt operator on $L^{2}(B)$ [Alt16, 10.16 p. $342 \mathrm{ff}$.]. This is equivalent to the first statement by Proposition B.3.

For the second statement we note that the bounds of the singularity (Equation 2.23) and (2.24) ) state that there exists a $C>0$ such that for all $\mathbf{x}, \mathbf{y} \in D$ with $\mathbf{x} \neq \mathbf{y}$

$$
|g(\mathbf{x}, \mathbf{y})| \leq C|\mathbf{x}-\mathbf{y}|^{-\frac{d-1}{2}}
$$

Because $B$ is bounded we can define $R:=\sup \left\{\left|\mathbf{y}_{1}-\mathbf{y}_{2}\right|: \mathbf{y}_{1}, \mathbf{y}_{2} \in B\right\}$ and set $\mathfrak{B}=$ $\left\{\mathbf{x} \in \mathbb{R}^{d}:|\mathbf{x}| \leq R\right\}$. Hence, we have the estimate

$$
h(\mathbf{y}) \leq C^{2} \int_{\mathfrak{B}}|\mathbf{x}|^{-(d-1)} d \mathbf{x} \quad \text { for every } \mathbf{y} \in B
$$

This yields the claim as the right hand side is finite and independent of $\mathbf{y}$.

\section{Fundamental solution property}

As already discussed above, for any fixed $\mathbf{y} \in \mathbb{R}^{d}, g(\cdot, \mathbf{y})$ (defined by 2.19 or 2.20 ) solves Equation (2.14) in the sense of distributions. With the differential operators $L_{k}$ (2.5) and $L_{k}^{\top}(2.6)$ the fundamental solution property is precisely formulated by the following proposition. 
Proposition 2.13 (Fundamental solution property - free field).

For any test function $\phi \in C_{c}^{\infty}\left(\mathbb{R}^{d}\right)$ and any $\mathbf{y} \in \mathbb{R}^{d}$, we have

$$
\int_{\mathbb{R}^{d}} g(\mathbf{x}, \mathbf{y})\left[L_{k}^{\top} \phi\right](\mathbf{x}) d \mathbf{x}=-\phi(\mathbf{y})
$$

Proof. For $\mathbf{m}=0$, the claim follows immediately from Green's formula (see CK13, p. 17 , p. 74]). Hence, the no-flow Green's function $g_{0}$ satisfies

$$
-\phi(\mathbf{y})=\int_{\mathbb{R}^{d}} g_{0}(\mathbf{x}, \mathbf{y}, k)\left[k^{2} \phi+\Delta \phi\right](\mathbf{x}) d \mathbf{x} .
$$

The fundamental solution property for $|\mathbf{m}|>0$ can be derived by means of the Lorentz transformation. With

$$
\gamma=\frac{|\mathbf{m}| i k}{\beta} \text { and } \quad \psi(\mathbf{x})=\exp \left(-\gamma[\mathbf{x}-\mathbf{T y}]_{1}\right) \phi\left(\mathbf{T}^{-1} \mathbf{x}\right)
$$

we have the identity

$$
\phi(\mathbf{x})=\exp \left(\frac{\gamma}{\beta}\left(x_{1}-y_{1}\right)\right) \psi(\mathbf{T x})
$$

Straightforward calculations show further that

$$
\left[L_{k}^{\top} \phi\right](\mathbf{x})=\exp \left(\frac{\gamma}{\beta}\left(x_{1}-y_{1}\right)\right)\left[\frac{k^{2}}{\beta^{2}} \psi+\Delta \psi\right](\mathbf{T x}) .
$$

Putting all these statements together yields

$$
\begin{aligned}
& \int_{\mathbb{R}^{d}} g(\mathbf{x}, \mathbf{y})\left[L_{k}^{\top} \phi\right](\mathbf{x}) d \mathbf{x}=\frac{1}{\beta} \int_{\mathbb{R}^{d}} \exp \left(-\frac{\gamma}{\beta}\left(x_{1}-y_{1}\right)\right) g_{0}\left(\mathbf{T} \mathbf{x}, \mathbf{T} \mathbf{y}, \frac{k}{\beta}\right)\left[L_{k}^{\top} \phi\right](\mathbf{x}) d \mathbf{x} \\
& =\frac{1}{\beta} \int_{\mathbb{R}^{d}} g_{0}\left(\mathbf{T} \mathbf{x}, \mathbf{T} \mathbf{y}, \frac{k}{\beta}\right)\left[\frac{k^{2}}{\beta^{2}} \psi+\Delta \psi\right](\mathbf{T x}) d \mathbf{x} \\
& =\int_{\mathbb{R}^{d}} g_{0}\left(\mathbf{x}, \mathbf{T y}, \frac{k}{\beta}\right)\left[\frac{k^{2}}{\beta^{2}} \psi+\Delta \psi\right](\mathbf{x}) d \mathbf{x}=-\psi(\mathbf{T y})=-\phi(\mathbf{y}) .
\end{aligned}
$$

\section{Representation formula}

A well-known result for the standard Helmholtz equation in two or three spatial dimensions is the so-called representation theorem. Again, this result can be carried over to the convected Helmholtz equation by means of the Lorentz transformation.

Theorem 2.14 (Representation theorem - free field).

Let $U \subset \mathbb{R}^{d}$ be a bounded domain of class $C^{2}$ and $u \in C^{2}\left(\mathbb{R}^{d} \backslash \bar{U}\right) \cap C^{1}\left(\mathbb{R}^{d} \backslash U\right)$ such that $L_{k} u=0$ in $\mathbb{R}^{d} \backslash \bar{U}$. Furthermore, assume that $v(\mathbf{y}):=\exp \left(\frac{|\mathbf{m}| i k}{\beta} x_{1}\right) u\left(\mathbf{T}^{-1} \mathbf{x}\right)$ satisfies 
the Sommerfeld radiation condition (2.15). Then we have the following representation formula for any $\mathbf{x} \in \mathbb{R}^{d} \backslash \bar{U}$

$$
\begin{aligned}
u(\mathbf{x})= & \oint_{\partial U}\left\{u(\mathbf{y}) \nabla_{\mathbf{y}} g(\mathbf{x}, \mathbf{y})-g(\mathbf{x}, \mathbf{y}) \nabla_{\mathbf{y}} u(\mathbf{y})\right\} \cdot \mathbf{n} d s(\mathbf{y}) \\
& +|\mathbf{m}|^{2} \oint_{\partial U}\left\{g(\mathbf{x}, \mathbf{y}) \frac{\partial u}{\partial \mathbf{y}_{1}}(\mathbf{y})-u(\mathbf{y}) \frac{\partial g}{\partial \mathbf{y}_{1}}(\mathbf{x}, \mathbf{y})\right\} n_{1} d s(\mathbf{y}) \\
& -2|\mathbf{m}| i k \oint_{\partial U} u(\mathbf{y}) g(\mathbf{x}, \mathbf{y}) n_{1} d s(\mathbf{y})
\end{aligned}
$$

where $\mathbf{n}=\left(n_{1}, \ldots, n_{d}\right)^{\top}$ is the outer unit normal vector of $\partial U$.

Proof. The proof is based on the Lorentz transformation and the corresponding result for the standard Helmholtz equation (see [CC06, Theorem 3.1 p. 52] and [CK13, Theorem 2.5 p.19]). Since it is quite technical, it is shifted to the Appendix A.

\subsection{Green's function in waveguides}

In this section we derive an explicit representation of the Green's function for a waveguide domain $D=\mathcal{A} \times \mathbb{R}$, where the cross section satisfies Assumption 2.5. The walls of the waveguide are assumed to be sound hard, i.e. on the boundary $\partial D$ we impose Neumann boundary conditions. Thus the sound pressure field $p$ generated by a source $Q$ satisfies

$$
\begin{aligned}
k^{2} p+2|\mathbf{m}| i k \frac{\partial}{\partial x_{1}} p+\beta^{2} \frac{\partial^{2}}{\partial x_{1}^{2}} p+\Delta_{\perp} p & =-Q \quad \text { in } D \\
\nabla p \cdot \mathbf{n} & =0 \quad \text { on } \partial D .
\end{aligned}
$$

As we seek to find a Green's function the source term $Q$ has to be chosen as a Dirac distribution i.e.

$$
Q\left(x_{1}, x_{\perp}\right)=\delta\left(x_{1}-y_{1}\right) \delta\left(x_{\perp}-y_{\perp}\right)
$$

for an arbitrary but fixed source position $\mathbf{y} \in D$. First we will formally derive a representation of the Green's function and then prove its essential properties. We consider a series expansion using the Neumann eigenfunctions $\psi_{n}$ from Theorem 2.6 in order to satisfy the boundary condition $2.26 \mathrm{~b}$

$$
g\left(x_{1}, x_{\perp}, y_{1}, y_{\perp}\right)=\sum_{n \in \mathbb{N}_{0}} c_{n}\left(x_{1}\right) \psi_{n}\left(x_{\perp}\right)
$$

The coefficient functions $c_{n}$ have to be determined.

\section{Standard Helmholtz equation}

Assuming $|\mathbf{m}|=0$ and formally inserting the modal series representation 2.27 into 
equation $2.26 \mathrm{a}$ leads to

$$
\sum_{n=0}^{\infty}\left(c_{n}^{\prime \prime}\left(x_{1}\right)+\left(k^{2}-\lambda_{n}\right) c_{n}\left(x_{1}\right)\right) \psi_{n}\left(x_{\perp}\right)=\sum_{n=0}^{\infty}-\psi_{n}\left(y_{\perp}\right) \delta\left(x_{1}-y_{1}\right) \psi_{n}\left(x_{\perp}\right)
$$

and we define

$$
k_{n}:=\sqrt{\left|k^{2}-\lambda_{n}\right|} .
$$

In order to exclude the resonance case $k_{n}=0$, we assume that the wavenumber $k$ satisfies 2.11) with $\beta=1$ i.e.

$$
k^{2} \notin\left\{\lambda_{n}: n \in \mathbb{N}_{0}\right\}
$$

Equating the coefficients in 2.28 yields an one dimensional ordinary differential equation with constant coefficients for each $n \in \mathbb{N}_{0}$

$$
c_{n}^{\prime \prime}\left(x_{1}\right)+\left(k^{2}-\lambda_{n}\right) c_{n}\left(x_{1}\right)=-\psi_{n}\left(y_{\perp}\right) \delta\left(x_{1}-y_{1}\right) .
$$

Due to linearity we may solve instead

$$
b_{n}^{\prime \prime}\left(x_{1}\right)+\left(k^{2}-\lambda_{n}\right) b_{n}\left(x_{1}\right)=\delta\left(x_{1}-y_{1}\right)
$$

and recover the original solution afterwards by $c_{n}\left(x_{1}\right)=-\psi_{n}\left(y_{\perp}\right) b_{n}\left(x_{1}\right)$.

To obtain a solution in the sense of distributions, we divide the real line into two parts: $\mathbb{R}_{<}=\left\{x \in \mathbb{R}: x<y_{1}\right\}$ and $\mathbb{R}_{>}=\left\{x \in \mathbb{R}: x>y_{1}\right\}$. We further assume that $b_{n} \in C^{2}\left(\mathbb{R}_{\lessgtr}\right) \cap C^{1}\left(\overline{\mathbb{R}_{\lessgtr}}\right)$. That yields solutions of the form

$$
b_{n}\left(x_{1}\right)=\frac{i}{2 \sqrt{\left(k^{2}-\lambda_{n}\right)}} e^{ \pm i \sqrt{\left(k^{2}-\lambda_{n}\right)}\left|x_{1}-y_{1}\right|} .
$$

Depending on the sign of $\left(k^{2}-\lambda_{n}\right)$, the coefficients $b_{n}$ are either of constant magnitude or grow resp. decay exponentially. In order to select physical meaningful solutions, we employ the radiation conditions from (SP2b). To this end, we make a distinction of cases.

\section{Case $k^{2}>\lambda_{n}$ (propagating modes):}

That case occurs finitely many times and we choose physical solutions

$$
b_{n}\left(x_{1}\right)=\frac{i}{2 k_{n}} e^{i k_{n}\left|x_{1}-y_{1}\right|}
$$

via the radiation condition

$$
\lim _{\left|x_{1}\right| \rightarrow \infty}\left(\frac{\partial}{\partial\left|x_{1}\right|}-i k_{n}\right) b_{n}\left(x_{1}\right)=0
$$




\section{Case $k^{2}<\lambda_{n}$ (decaying modes):}

That case occurs infinitely many times and we choose physical solutions

$$
b_{n}\left(x_{1}\right)=\frac{1}{2 k_{n}} e^{-k_{n}\left|x_{1}-y_{1}\right|}
$$

that do not tend to infinity for $\left|x_{1}\right| \rightarrow \infty$. In terms of the radiation condition SP2b) this is the choice for which $\lim \sup \left|b_{n}\left(x_{1}\right)\right|<\infty$.

$$
\left|x_{1}\right| \rightarrow \infty
$$

With the number of propagating modes

$$
n_{\text {prop }}=\sup \left\{n \in \mathbb{N}_{0}:\left(k^{2}-\lambda_{n}\right)>0\right\}
$$

the no-flow Green's function reads as

$$
\begin{aligned}
g_{0}\left(x_{1}, x_{\perp}, y_{1}, y_{\perp}, k\right) & =\frac{-i}{2} \sum_{n=0}^{n_{\text {prop }}} \frac{1}{k_{n}} e^{i k_{n}\left|x_{1}-y_{1}\right|} \psi_{n}\left(y_{\perp}\right) \psi_{n}\left(x_{\perp}\right) \\
& -\frac{1}{2} \sum_{n=n_{\text {prop }}+1}^{\infty} \frac{1}{k_{n}} e^{-k_{n}\left|x_{1}-y_{1}\right|} \psi_{n}\left(y_{\perp}\right) \psi_{n}\left(x_{\perp}\right) .
\end{aligned}
$$

\section{Convected Helmholtz equation}

For $|\mathbf{m}|>0$ denote by $g_{\mathbf{m}}\left(x_{1}, x_{\perp}, y_{1}, y_{\perp}, k\right)$ the Green's function for Equations 2.26a and (2.26b). Via the Lorentz transformation (Proposition 2.1) it is given by

$$
g_{\mathbf{m}}\left(x_{1}, x_{\perp}, y_{1}, y_{\perp}, k\right)=\frac{1}{\beta} \exp \left(\frac{-i k|\mathbf{m}|}{\beta^{2}}\left(x_{1}-y_{1}\right)\right) g_{0}\left(\frac{x_{1}}{\beta}, x_{\perp}, \frac{y_{1}}{\beta}, y_{\perp}, \frac{k}{\beta}\right) .
$$

For the case with convection, we assume that (2.11) (avoiding the resonance case) is satisfied i.e. $\frac{k^{2}}{\beta^{2}}-\lambda_{n} \neq 0$ for all $n \in \mathbb{N}_{0}$. For an explicit representation of 2.34), we define

$$
\begin{aligned}
s_{n} & =\sqrt{\left|k^{2}-\beta^{2} \lambda_{n}\right|}, \\
n_{\text {prop }} & =\sup \left\{n \in \mathbb{N}_{0}: k^{2}>\beta^{2} \lambda_{n}\right\} .
\end{aligned}
$$

That yields

$$
\begin{aligned}
& g_{\mathbf{m}}\left(x_{1}, x_{\perp}, y_{1}, y_{\perp}, k\right) \\
& =\frac{-i}{2} \exp \left(\frac{-i k|\mathbf{m}|}{\beta^{2}}\left(x_{1}-y_{1}\right)\right) \sum_{n=0}^{n_{\text {prop }}} \frac{1}{s_{n}} \exp \left(i \frac{s_{n}}{\beta^{2}}\left|x_{1}-y_{1}\right|\right) \psi_{n}\left(y_{\perp}\right) \psi_{n}\left(x_{\perp}\right) \\
& \quad-\frac{1}{2} \exp \left(\frac{-i k|\mathbf{m}|}{\beta^{2}}\left(x_{1}-y_{1}\right)\right) \sum_{n=n_{\text {prop }}+1}^{\infty} \frac{1}{s_{n}} \exp \left(-\frac{s_{n}}{\beta^{2}}\left|x_{1}-y_{1}\right|\right) \psi_{n}\left(y_{\perp}\right) \psi_{n}\left(x_{\perp}\right) .
\end{aligned}
$$


For the remainder of this section, we will prove similar results for the Green's function in waveguides as for the Green's function in free space.

\section{Integrability}

The waveguide Green's function (2.37) satisfies two similar integrability properties as in free space (cf. Proposition 2.12).

Proposition 2.15 (Hilbert-Schmidt kernel - waveguide).

For any open, bounded set $B \subset D$ the Green's function is square integrable on $B \times B$ i.e.

$$
\int_{B} \int_{B}|g(\mathbf{x}, \mathbf{y})|^{2} d \mathbf{x} d \mathbf{y}<\infty .
$$

If moreover, the eigenfunctions $\psi_{n}$ from Theorem 2.6 satisfy Assumption 2.8, then the auxiliary function

$$
h_{B}(\mathbf{y}):=\int_{B}|g(\mathbf{x}, \mathbf{y})|^{2} d \mathbf{x}
$$

belongs to $L^{\infty}(B)$.

Proof. We start with the proof of the first statement (2.38). For the sum over the first $n_{\text {prop }}+2$ summands, the statement is true, since the sum is finite and all summands are bounded functions. Thus it is sufficient to consider the decaying part starting at $n=n_{\text {prop }}+2$

$$
g^{\mathrm{dec}}\left(x_{1}, x_{\perp}, y_{1}, y_{\perp}\right):=\sum_{n=n_{\text {prop }}+2}^{\infty} c_{n}\left(x_{1}, y_{1}\right) \psi_{n}\left(y_{\perp}\right) \psi_{n}\left(x_{\perp}\right)
$$

with the streamwise coefficients

$$
c_{n}\left(x_{1}, y_{1}\right)=\frac{-1}{2 s_{n}} \exp \left(-\frac{s_{n}}{\beta^{2}}\left|x_{1}-y_{1}\right|\right) \exp \left(\frac{-i k|\mathbf{m}|}{\beta^{2}}\left(x_{1}-y_{1}\right)\right) .
$$

A sufficient condition for 2.38 is that for any bounded interval $I=[-R, R]$ the iterated integral

$$
I^{\mathrm{dec}}:=\int_{I \times I} \int_{\mathcal{A} \times \mathcal{A}}\left|g^{\mathrm{dec}}\left(x_{1}, x_{\perp}, y_{1}, y_{\perp}\right)\right|^{2} d\left(x_{\perp}, y_{\perp}\right) d\left(x_{1}, x_{2}\right)
$$

is finite. Note that the functions $\varphi_{n}\left(x_{\perp}, y_{\perp}\right)=\psi_{n}\left(x_{\perp}\right) \psi_{n}\left(y_{\perp}\right)$ form an orthonormal system on $L^{2}(\mathcal{A} \times \mathcal{A})$. Now let $x_{1}, y_{1}$ with $x_{1} \neq y_{1}$ be arbitrary but fixed. Further we obtain from Parsevals equation (for the Hilbert space $\mathcal{H}=\overline{\operatorname{span}\left\{\varphi_{n}\right\}}$ )

$$
\left\|g^{\mathrm{dec}}\left(x_{1}, \cdot, y_{1}, \cdot\right)\right\|_{L^{2}(\mathcal{A} \times \mathcal{A})}^{2}=\sum_{n=n_{\text {prop }}+2}^{\infty}\left|c_{n}\left(x_{1}, y_{1}\right)\right|^{2} .
$$

Hence, the function $g^{\operatorname{dec}}\left(x_{1}, \cdot, y_{1}, \cdot\right)$ belongs to $L^{2}(\mathcal{A} \times \mathcal{A})$ if we can show that the right 
hand side is finite. By Weyl's Law, the eigenvalues $\lambda_{n}$ grow at least as fast as $n$ (see Proposition A.1 i.e. there exists a constant $C_{\text {Weyl }}>0$ such that

$$
C_{\text {Weyl }} \cdot n \leq \lambda_{n} \quad \text { for all } n \in \mathbb{N}_{0} \text {. }
$$

This implies the following bound for $n>n_{\text {prop }}$

$$
\frac{1}{s_{n}}=\frac{1}{\sqrt{\left|k^{2}-\beta^{2} \lambda_{n}\right|}}=\frac{1}{\sqrt{\lambda_{n}}} \frac{1}{\sqrt{\left|\beta^{2}-\frac{k^{2}}{\lambda_{n}}\right|}} \leq B_{1} \frac{1}{\sqrt{n}}
$$

with the constant

$$
B_{1}:=\frac{1}{\sqrt{C_{\text {Weyl }}}} \sup _{n>n_{\text {prop }}} \frac{1}{\sqrt{\left|\beta^{2}-\frac{k^{2}}{\lambda_{n}}\right|}} .
$$

For the exponential term we obtain similarly

$$
\begin{aligned}
\exp \left(\frac{-2 s_{n}}{\beta^{2}}\left|x_{1}-y_{1}\right|\right) & =\exp \left(\frac{-2 \sqrt{\lambda_{n}} \sqrt{\left|\beta^{2}-\frac{k^{2}}{\lambda_{n}}\right|}}{\beta^{2}}\left|x_{1}-y_{1}\right|\right) \\
& \leq \exp \left(-B_{2} \sqrt{n}\left|x_{1}-y_{1}\right|\right)
\end{aligned}
$$

with the constant

$$
B_{2}:=\frac{2 \sqrt{C_{\text {Weyl }}}}{\beta^{2}} \inf _{n>n_{\text {prop }}} \sqrt{\left|\beta^{2}-\frac{k^{2}}{\lambda_{n}}\right|}
$$

Thus, we obtain using the estimates 2.42 and 2.43

$$
\begin{aligned}
\sum_{n=n_{\text {prop }}+2}^{\infty}\left|c_{n}\left(x_{1}, y_{1}\right)\right|^{2} & =\sum_{n=n_{\text {prop }+2}}^{\infty} \frac{1}{s_{n}^{2}} \exp \left(\frac{-2 s_{n}}{\beta^{2}}\left|x_{1}-y_{1}\right|\right) \\
& \leq B_{1}^{2} \sum_{n=n_{\text {prop }+2}}^{\infty} \frac{1}{n} \exp \left(-B_{2}\left|x_{1}-y_{1}\right| \sqrt{n}\right) \\
& \leq B_{1}^{2} \int_{1}^{\infty} \frac{1}{z} \exp \left(-B_{2}\left|x_{1}-y_{1}\right| \sqrt{z}\right) d z
\end{aligned}
$$

That implies

$$
I^{\mathrm{dec}} \leq B_{1}^{2} \int_{1}^{\infty} \frac{1}{z}\left[\int_{I \times I} \exp \left(-B_{2}\left|x_{1}-y_{1}\right| \sqrt{z}\right) d x_{1} d y_{1}\right] d z .
$$


The inner integral can be estimated by the fact that

$$
\begin{aligned}
& \int_{-R}^{R} \exp \left(-B_{2}\left|x_{1}-y_{1}\right| \sqrt{z}\right) d x_{1} \\
= & \frac{2}{B_{2} \sqrt{z}}-\frac{1}{B_{2} \sqrt{z}}\left\{\exp \left(B_{2}\left(R+y_{1}\right) \sqrt{z}\right)+\exp \left(-B_{2}\left(R-y_{1}\right) \sqrt{z}\right)\right\} \\
\leq & \frac{2}{B_{2} \sqrt{z}}
\end{aligned}
$$

and hence

$$
[\ldots] \leq \frac{4 R}{B_{2} \sqrt{z}}
$$

Altogether we obtain

$$
I^{\mathrm{dec}} \leq \frac{4 R B_{1}^{2}}{B_{2}} \int_{1}^{\infty} \frac{1}{z^{3 / 2}} d z<\infty
$$

This proves 2.38).

For the second statement we note that there exists a bounded interval $I=[-R, R]$ such that

$$
h_{B}(\mathbf{y}) \leq \int_{I}\left\|g\left(x_{1}, \cdot, y_{1}, y_{\perp}\right)\right\|_{L^{2}(\mathcal{A})}^{2} d x_{1}
$$

To bound the last expression from above, it is sufficient to consider only the sum over the decaying modes starting at $n=n_{\text {prop }}+2$ (cf. Equation 2.40). Parseval's equality yields

$$
\left\|g^{\mathrm{dec}}\left(x_{1}, \cdot, y_{1}, y_{\perp}\right)\right\|_{L^{2}(\mathcal{A})}^{2}=\sum_{n=n_{\text {prop }}+2}^{\infty}\left|c_{n}\left(x_{1}, y_{1}\right)\right|^{2}\left|\psi_{n}\left(y_{\perp}\right)\right|^{2} .
$$

Since $\psi_{n}\left(y_{\perp}\right)$ is uniformly bounded independent from $n$ and $y_{\perp}$ there exits a $C>0$ such that

$$
h_{B}(\mathbf{y}) \leq C \int_{I} \sum_{n=n_{\text {prop }}+2}^{\infty}\left|c_{n}\left(x_{1}, y_{1}\right)\right|^{2} d x_{1} .
$$

Now we can conclude similar as in the proof of the first statement (cf. Equation 2.44) 
and obtain

$$
\begin{aligned}
h_{B}(\mathbf{y}) & \leq C \int_{I} \sum_{n=n_{\text {prop }}+2}^{\infty}\left|c_{n}\left(x_{1}, y_{1}\right)\right|^{2} d x_{1} \\
& \leq C B_{1}^{2} \int_{I} \int_{1}^{\infty} \frac{1}{z} \exp \left(-B_{2}\left|x_{1}-y_{1}\right| \sqrt{z}\right) d z d x_{1} \\
& \leq \frac{2 C B_{1}^{2}}{B_{2}} \int_{1}^{\infty} \frac{1}{z^{3 / 2}} d z<\infty .
\end{aligned}
$$

Hence $h_{B} \in L^{\infty}(B)$.

\section{Fundamental solution property}

In order to prove the fundamental solution property of the Green's function in waveguides, we start with a useful lemma on the representation of test functions.

Lemma 2.16 (Series representation for test functions).

Assume the eigenfunctions $\psi_{n}$ from Theorem 2.6 satisfy Assumption 2.8. Let further $\phi \in V^{\mathrm{Neu}}=\left\{\phi \in C_{c}^{\infty}(\bar{D}): \frac{\partial \phi}{\partial n}=0\right.$ on $\left.\partial \mathcal{A}\right\}$ and define the coefficients

$$
a_{n}\left(x_{1}\right)=\int_{\mathcal{A}} \phi\left(x_{1}, x_{\perp}\right) \psi_{n}\left(x_{\perp}\right) d x_{\perp} \quad \text { for } n \in \mathbb{N}_{0}
$$

Then there exists a constant $C$ independent of $n$ such that the derivatives $a_{n}^{(l)}$ up to order 2 satisfy

$$
\max _{l=0,1,2} \sup _{x_{1} \in \mathbb{R}}\left|a_{n}^{(l)}\left(x_{1}\right)\right| \leq C \frac{1}{\lambda_{n}^{3 / 2}} \quad \text { for } n \geq 1
$$

Moreover, we have

(i) for every $\mathbf{x} \in D$ :

$$
\phi(\mathbf{x})=\sum_{n \in \mathbb{N}_{0}} a_{n}\left(x_{1}\right) \psi_{n}\left(x_{\perp}\right)
$$

(ii) for every $x_{1} \in \mathbb{R}$ :

$$
\left[L_{k}^{\top} \phi\right]\left(x_{1}, \cdot\right)=\sum_{n \in \mathbb{N}_{0}}\left[\left(k^{2}-\lambda_{n}\right) a_{n}\left(x_{1}\right)-2|\mathbf{m}| i k a_{n}^{\prime}\left(x_{1}\right)+\beta^{2} a_{n}^{\prime \prime}\left(x_{1}\right)\right] \psi_{n}(\cdot) \quad \text { in } L^{2}(\mathcal{A}) .
$$

Proof. See Appendix A.

Similarly to Proposition 2.13 , we can now formulate the fundamental solution property for the Green's function in waveguides explicitly. 
Proposition 2.17 (Fundamental solution property - waveguide).

For any test function $\phi \in V^{\mathrm{Neu}}$ and any $\mathbf{y} \in D$, we have

$$
\int_{D} g(\mathbf{x}, \mathbf{y})\left[L_{k}^{\top} \phi\right](\mathbf{x}) d \mathbf{x}=-\phi(\mathbf{y})
$$

Proof. Firstly, note that the integral expression is well defined as for all $\mathbf{y}$ and $\phi \in V^{\mathrm{Neu}}$ we can find a bounded set $B=[-R, R] \times \mathcal{A}$ such that

$$
\int_{B}|g(\mathbf{x}, \mathbf{y})|^{2} d \mathbf{x}<\infty
$$

by the second statement of Proposition 2.15. Now keep $\phi \in V^{\text {Neu }}$ and $\mathbf{y} \in D$ arbitrary but fixed and choose an $R>\left|y_{1}\right| \operatorname{such}$ that $\operatorname{supp}(\phi) \subset[-R, R] \times \mathcal{A}$. We get

$$
\begin{aligned}
& \int_{D} g\left(x_{1}, x_{\perp}, y_{1}, y_{\perp}\right)\left(L_{k}^{\top} \phi\right)\left(x_{1}, x_{\perp}\right) d\left(x_{1}, x_{\perp}\right) \\
= & \int_{-R}^{R} \int_{\mathcal{A}} g\left(x_{1}, x_{\perp}, y_{1}, y_{\perp}\right)\left(L_{k}^{\top} \phi\right)\left(x_{1}, x_{\perp}\right) d x_{\perp} d x_{1} .
\end{aligned}
$$

By Lemma 2.16 we have for every $x_{1} \in \mathbb{R}$

$$
\left[L_{k}^{\top} \phi\right]\left(x_{1}, \cdot\right)=\sum_{n \in \mathbb{N}_{0}}\left[\left(k^{2}-\lambda_{n}\right) a_{n}\left(x_{1}\right)-2|\mathbf{m}| i k a_{n}^{\prime}\left(x_{1}\right)+\beta^{2} a_{n}^{\prime \prime}\left(x_{1}\right)\right] \psi_{n}(\cdot) \quad \text { in } L^{2}(\mathcal{A}) .
$$

Using the separated Green's functions

$$
c_{n}\left(x_{1}, y_{1}\right)=\frac{-1}{2 s_{n}} \exp \left(\frac{i k|\mathbf{m}|}{\beta^{2}}\left(x_{1}-y_{1}\right)\right) \cdot \begin{cases}i \cdot \exp \left(i \frac{s_{n}}{\beta^{2}}\left|x_{1}-y_{1}\right|\right) & \text { for } n \leq n_{\text {prop }} \\ \exp \left(-\frac{s_{n}}{\beta^{2}}\left|x_{1}-y_{1}\right|\right) & \text { for } n>n_{\text {prop }}\end{cases}
$$

we can replace $g\left(x_{1}, \cdot, y_{1}, y_{\perp}\right)$ and $\left[L_{k}^{\top} \phi\right]\left(x_{1}, \cdot\right)$ in the inner integral of 2.45 by their series representation. This yields

$$
\begin{aligned}
& \int_{D} g\left(x_{1}, x_{\perp}, y_{1}, y_{\perp}\right)\left(L_{k}^{\top} \phi\right)\left(x_{1}, x_{\perp}\right) d\left(x_{1}, x_{\perp}\right) \\
= & \int_{-R}^{R} \sum_{n \in \mathbb{N}_{0}} \psi_{n}\left(y_{\perp}\right) c_{n}\left(x_{1}, y_{1}\right)\left(D_{k, n}^{\top} a_{n}\right)\left(x_{1}\right) d x_{1},
\end{aligned}
$$

with the differential operators

$$
\begin{aligned}
D_{k, n} & :=\left(k^{2}-\lambda_{n}\right)+2|\mathbf{m}| i k \frac{\partial}{\partial x_{1}}+\beta^{2} \frac{\partial^{2}}{\partial x_{1}^{2}} \\
D_{k, n} & :=\left(k^{2}-\lambda_{n}\right)-2|\mathbf{m}| i k \frac{\partial}{\partial x_{1}}+\beta^{2} \frac{\partial^{2}}{\partial x_{1}^{2}} .
\end{aligned}
$$


Note that

$$
\sum_{n \in \mathbb{N}_{0}} \psi_{n}\left(y_{\perp}\right) c_{n}\left(x_{1}, y_{1}\right)\left(D_{k, n}^{\top} a_{n}\right)\left(x_{1}\right)
$$

converges pointwise almost everywhere in $[-R, R]$ and has an integrable majorant $m \in$ $L^{1}(-R, R)$. To construct a suitable $m$ we choose $N \geq n_{\text {prop }}+2$ sufficiently large such that for all $n \geq N$

$$
\max \left\{\left|k^{2}-\lambda_{n}\right|, 2|\mathbf{m}| k, \beta^{2}\right\} \leq \lambda_{n} .
$$

Now we can find positive constants $C_{1}, C_{2}, C_{3}, C_{4}$ independent of $n$ and $x_{1}$ such that for all $n \geq N$

$$
\begin{aligned}
& \sup _{x_{1} \in \mathbb{R}}\left|\left(D_{k, n}^{\top} a_{n}\right)\left(x_{1}\right)\right| \leq C_{1} \frac{1}{\sqrt{n}}, \\
& \sup _{y_{\perp} \in \mathcal{A}}\left|\psi_{n}\left(y_{\perp}\right)\right| \leq C_{2}, \\
& \left|c_{n}\left(x_{1}, y_{1}\right)\right| \leq \frac{C_{3}}{\sqrt{n}} \exp \left(-C_{4} \sqrt{n}\left|x_{1}-y_{1}\right|\right) .
\end{aligned}
$$

Here we have used (2.47), Lemma 2.16. Assumption 2.8 and the decay properties of the eigenvalues similar as in the proof of Proposition 2.15. A majorant $m$ is then given by

$$
m\left(x_{1}\right)=\sum_{n=0}^{N-1}\left|\psi_{n}\left(y_{\perp}\right) c_{n}\left(x_{1}, y_{1}\right)\left(D_{k, n}^{\top} a_{n}\right)\left(x_{1}\right)\right|+C_{1} C_{2} C_{3} \sum_{n=N}^{\infty} \frac{1}{n} \exp \left(-C_{4} \sqrt{n}\left|x_{1}-y_{1}\right|\right) .
$$

Moreover, the $c_{n}$ satisfy

$$
\left(D_{k, n} c_{n}\right)\left(x_{1}, y_{1}\right)=-\delta\left(x_{1}-y_{1}\right)
$$

in the sense of distributions. Hence we conclude by interchanging summation and integration in (2.46)

$$
\begin{aligned}
& \int_{D} g\left(x_{1}, x_{\perp}, y_{1}, y_{\perp}\right)\left(L_{k}^{\top} \phi\right)\left(x_{1}, x_{\perp}\right) d\left(x_{1}, x_{\perp}\right) \\
= & \sum_{n \in \mathbb{N}_{0}} \psi_{n}\left(y_{\perp}\right) \int_{-R}^{R} c_{n}\left(x_{1}, y_{1}\right)\left(D_{k, n}^{\top} a_{n}\right)\left(x_{1}\right) d x_{1} \\
= & -\sum_{n \in \mathbb{N}_{0}} a_{n}\left(y_{1}\right) \psi_{n}\left(y_{\perp}\right)=-\phi(\mathbf{y}) .
\end{aligned}
$$

Here we have used the series representation of $\phi$ from Lemma 2.16 in the last line.

\subsection{Solution of the deterministic source problem}

Using the Green's functions that were presented in the last two sections we can now construct solutions of the deterministic source problem in free space $($ SP1a + SP1b $)$ 
and waveguides $($ SP2a + SP2b $)$.

We define the following functions and operators, which are well defined due to the Hilbert-Schmidt property of the Green's function (cf. Proposition 2.12 and 2.15)

- The volume potential $w_{s}$ of a function $s \in L^{2}(D)$ with compact support

$$
w_{s}(\mathbf{x}):=\int_{D} g(\mathbf{x}, \mathbf{y}) s(\mathbf{y}) d \mathbf{y} \quad \text { for } \mathbf{x} \in D .
$$

- $S: C_{c}^{\infty}(\bar{D}) \subset L^{2}(D) \rightarrow L_{\mathrm{loc}}^{2}(D)$

$$
(S \phi)(\mathbf{y}):=\int_{D} g(\mathbf{x}, \mathbf{y}) \phi(\mathbf{x}) d \mathbf{x} \quad \text { for } \mathbf{y} \in D .
$$

Where $S$ is a possibly unbounded, densely defined linear operator.

We start with some calculation rules that will be useful for the proofs within this section. Recall that for any linear operator $T: \mathcal{H}_{1} \rightarrow \mathcal{H}_{2}$ between two Hilbert spaces, the transposed operator $T^{\top}: \mathcal{H}_{2} \rightarrow \mathcal{H}_{1}$ is defined by

$$
T^{\top} u:=\overline{T^{*}(\bar{u})}
$$

and for any $u \in \operatorname{dom}\left(T^{*}\right), v \in \operatorname{dom}(T)$ we have

$$
\left\langle T^{\top} u, v\right\rangle_{\mathcal{H}_{2}}=\langle u, \overline{T \bar{v}}\rangle_{\mathcal{H}_{1}} .
$$

With a slight abuse of notation we can extend these relations to the operator $S$.

Definition \& Proposition 2.18 (The operators $S^{\star}$ and $S^{\top}$ ).

Define the set

$$
L_{c}^{2}(D):=\left\{v \in L^{2}(D): v \text { has compact support }\right\} .
$$

The linear operators $S^{\star}: L_{c}^{2}(D) \subset L^{2}(D) \rightarrow L_{\mathrm{loc}}^{2}(D)$

$$
\left(S^{\star} v\right)(\mathbf{x}):=\int_{D} \overline{g(\mathbf{x}, \mathbf{y})} v(\mathbf{y}) d \mathbf{y}
$$

and $S^{\top}: L_{c}^{2}(D) \subset L^{2}(D) \rightarrow L_{\mathrm{loc}}^{2}(D)$

$$
\left(S^{\top} v\right):=\overline{\left(S^{\star} \bar{v}\right)}
$$

are well defined. Furthermore, for every $u \in L_{c}^{2}(D)$ and $v \in L_{c}^{2}(D) \cap L^{\infty}(D)$ we have

$$
\left\langle S^{\top} u, v\right\rangle_{L^{2}(D)}=\langle u, \overline{S \bar{v}}\rangle_{L^{2}(D)} .
$$


Proof. Let $v \in L_{c}^{2}(D)$ then for every compact set $K \subset D$

$$
\int_{K}\left|\left(S^{\star} v\right)(\mathbf{x})\right|^{2} d \mathbf{x} \leq\|v\|_{L^{2}(K)}^{2} \int_{K} \int_{K}|g(\mathbf{x}, \mathbf{y})|^{2} d \mathbf{x} d \mathbf{y}<\infty
$$

since $g$ is square integrable. This proves the well definedness of $S^{\star}$ and $S^{\top}$. Now fix $u, v \in L_{c}^{2}(D)$ with $v \in L^{\infty}(D)$ and take a compact set $K$ with $(\operatorname{supp}(u) \cup \operatorname{supp}(v)) \subset K$. We compute

$$
\begin{array}{r}
\left\langle S^{\top} u, v\right\rangle_{L^{2}(D)}=\int_{D} \overline{\left(\int_{D} \overline{g(\mathbf{x}, \mathbf{y}) u(\mathbf{y})} d \mathbf{y}\right)} \overline{v(\mathbf{x})} d \mathbf{x}=\int_{K}\left(\int_{K} g(\mathbf{x}, \mathbf{y}) u(\mathbf{y}) d \mathbf{y}\right) \overline{v(\mathbf{x})} d \mathbf{x} \\
\stackrel{\text { Fubini-Tonelli }}{=} \int_{K} u(\mathbf{y})\left(\int_{K} g(\mathbf{x}, \mathbf{y}) \overline{v(\mathbf{x})} d \mathbf{x}\right) d \mathbf{y}=\langle u, \overline{S \bar{v}}\rangle_{L^{2}(D)}
\end{array}
$$

Here the Fubini-Tonelli theorem (cf. Theorem B.1) is applicable, since

$$
\int_{K}\left(\int_{K}|g(\mathbf{x}, \mathbf{y}) u(\mathbf{y})| d \mathbf{y}\right)|v(\mathbf{x})| d \mathbf{x} \leq \sqrt{|K|}\|v\|_{L^{\infty}(K)}\|u\|_{L^{2}(K)}\left(\int_{K} \int_{K}|g(\mathbf{x}, \mathbf{y})|^{2} d \mathbf{y} d \mathbf{x}\right)^{1 / 2}
$$

and the upper bound is finite. This proves the statement 2.51).

Note also that for any $s \in L_{c}^{2}(D)$, the volume potential $w_{s}$ satisfies

$$
w_{s}=S^{\top} s
$$

With the introduced tools we can now prove the existence of solutions of the deterministic source problems introduced in Section $2.2($ SP1a + SP1b and $(\mathrm{SP} 2 \mathrm{a})+\mathrm{SP} 2 \mathrm{~b})$, which are moreover unique by Theorem 2.4 and Theorem 2.10 .

Theorem 2.19 (Solution of the forward source problem - free space).

Let $s \in L_{c}^{2}\left(\mathbb{R}^{d}\right)$, then the volume potential $w_{s}$ (2.48) is the unique solution of the source problem $(\mathrm{SP} 1 \mathrm{a})+\mathrm{SP} 1 \mathrm{~b})$.

Proof. Firstly, $w_{s}$ belongs to the trial space $L_{\text {loc }}^{2}\left(\mathbb{R}^{d}\right)$ due to 2.52 . For the variational property SP1a we have to show that for all $\phi \in C_{c}^{\infty}\left(\mathbb{R}^{d}\right)$

$$
\int_{\mathbb{R}^{d}} w_{s} L_{k}^{\top} \phi d \mathbf{x}=-\int_{\mathbb{R}^{d}} s \phi d \mathbf{x} .
$$

Recall that the fundamental solution property of the Green's function (Proposition 2.13) directly implies that

$$
S\left(L_{k}^{\top} \phi\right)=-\phi \quad \text { for all } \phi \in C_{c}^{\infty}\left(\mathbb{R}^{d}\right)
$$


We compute

$$
\begin{gathered}
\int_{\mathbb{R}^{d}} w_{s} L_{k}^{\top} \phi d \mathbf{x} \stackrel{\sqrt[2.52]{-}}{=}\left\langle S^{\top} s, \overline{L_{k}^{\top} \phi}\right\rangle_{L^{2}\left(\mathbb{R}^{d}\right)} \stackrel{\sqrt[2.51]{-}}{=}\left\langle s, \overline{S\left(L_{k}^{\top} \phi\right)}\right\rangle_{L^{2}\left(\mathbb{R}^{d}\right)} \\
\stackrel{2.54)}{-}-\langle s, \bar{\phi}\rangle_{L^{2}\left(\mathbb{R}^{d}\right)}=-\int_{\mathbb{R}^{d}} s \phi d \mathbf{x},
\end{gathered}
$$

which proves 2.53 . For the radiation condition consider the back-transformation $b_{w_{s}}$ and apply a change of variables

$$
\begin{aligned}
b_{w_{s}}(\mathbf{x}) & =\exp \left(\frac{|\mathbf{m}| i k}{\beta} x_{1}\right) \int_{\mathbb{R}^{d}} g\left(\mathbf{T}^{-1} \mathbf{x}, \mathbf{y}\right) s(\mathbf{y}) d \mathbf{y} \\
& =\beta \exp \left(\frac{|\mathbf{m}| i k}{\beta} x_{1}\right) \int_{\mathbb{R}^{d}} g\left(\mathbf{T}^{-1} \mathbf{x}, \mathbf{T}^{-1} \mathbf{y}\right) s\left(\mathbf{T}^{-1} \mathbf{y}\right) d \mathbf{y} \\
= & \int_{\mathbb{R}^{d}} g_{0}\left(\mathbf{x}, \mathbf{y}, \frac{k}{\beta}\right) s\left(\mathbf{T}^{-1} \mathbf{y}\right) d \mathbf{y},
\end{aligned}
$$

where $g_{0}\left(\mathbf{x}, \mathbf{y}, \frac{k}{\beta}\right)$ denotes the Green's function for the standard Helmholtz equation at wavenumber $\frac{k}{\beta}$ (cf. 2.18). Now fix a ball $B$ such that $0 \in B$ and $\operatorname{supp}\left(s \circ \mathbf{T}^{-1}\right) \subset B$. For $\mathbf{x} \in \mathbb{R} \backslash \bar{B}$ we can differentiate under the integral which yields

$|\mathbf{x}|^{\frac{d-1}{2}}\left(\frac{\partial b_{w_{s}}}{\partial|\mathbf{x}|}-i \frac{k}{\beta} b_{w_{s}}\right)=|\mathbf{x}|^{\frac{d-1}{2}} \int_{B}\left\{\nabla_{\mathbf{x}} g_{0}\left(\mathbf{x}, \mathbf{y}, \frac{k}{\beta}\right) \cdot \frac{\mathbf{x}}{|\mathbf{x}|}-i \frac{k}{\beta} g_{0}\left(\mathbf{x}, \mathbf{y}, \frac{k}{\beta}\right)\right\} s\left(\mathbf{T}^{-1} \mathbf{y}\right) d \mathbf{y}$.

Since $g_{0}$ is a radiating solution of the standard Helmholtz equation, the right hand side converges to 0 uniformly for all directions $\frac{\mathbf{x}}{|\mathbf{x}|}$ and $\mathbf{y} \in B$ as $|x| \rightarrow \infty$ (see e.g. [Kir11, Section 6.2] for $d=3$ and [CK13, Section 3.4] for $d=2$ ). This completes the proof since there exists at most one solution by Theorem 2.4 .

Theorem 2.20 (Solution of the forward source problem - waveguide).

Let $D=\mathbb{R} \times \mathcal{A}$, where $\mathcal{A} \subset \mathbb{R}^{d-1}$ satisfies Assumption 2.5 and assume the eigenfunctions $\psi_{n}$ satisfy Assumption 2.8. Moreover, let $s \in L_{c}^{2}(D)$ and (2.11) be satisfied. Then the volume potential $w_{s}(2.48)$ is the unique solution of the source problem (SP2a + SP2b).

Proof. Again it is sufficient to show the solution properties SP2a + SP2b since the uniqueness follows by Theorem 2.10. Note that for a waveguide geometry we had the test space (cf. Equation 2.7)

$$
V^{\mathrm{Neu}}=\left\{\phi \in C_{c}^{\infty}(\bar{D}): \frac{\partial \phi}{\partial n}=0 \text { on } \partial \mathcal{A}\right\}
$$

The variational property $\mathrm{SP} 2 \mathrm{a}$ ) follows completely similar to the proof for the free space problem since by the fundamental solution property for the Green's function in waveguides (Proposition 2.17) we have

$$
S\left(L_{k}^{\top} \phi\right)=-\phi \quad \text { for all } \phi \in V^{\mathrm{Neu}} .
$$


It remains to show the radiation condition (SP2b). Similar as in the free space case we have a relationship of the Green's fuctions $g, g_{0}$ (see (2.34) ) and the back-transformation $b_{w_{s}}$ can be represented by

$$
b_{w_{s}}(\mathbf{x})=\int_{D} g_{0}\left(\mathbf{x}, \mathbf{y}, \frac{k}{\beta}\right) s\left(\mathbf{T}^{-1} \mathbf{y}\right) d \mathbf{y} .
$$

Now fix $R>0$ such that $\operatorname{supp}\left(s \circ \mathbf{T}^{-1}\right) \subset[-R, R] \times \overline{\mathcal{A}}$. For $n \in \mathbb{N}_{0}$ and for almost every $x_{1} \in \mathbb{R}$ the modal coefficients $b_{w_{s}}^{(n)}$ are given by

$$
b_{w_{s}}^{(n)}\left(x_{1}\right)=\int_{\mathcal{A}} b_{w_{s}}\left(x_{1}, x_{\perp}\right) \psi_{n}\left(x_{\perp}\right) d x_{\perp}=\int_{-R}^{R} \int_{\mathcal{A}} s\left(\beta y_{1}, y_{\perp}\right) c_{n}\left(x_{1}, y_{1}\right) \psi_{n}\left(y_{\perp}\right) d y_{\perp} d y_{1},
$$

where the functions $c_{n}$ are now given by the no-flow Green's function $g_{0}$ (cf. Equation (2.33))

$$
c_{n}\left(x_{1}, y_{1}\right)=\exp \left(i \sqrt{\frac{k^{2}}{\beta^{2}}-\lambda_{n}}\left|x_{1}-y_{1}\right|\right) \cdot\left\{\begin{array}{ll}
-\frac{i}{2} \frac{1}{\sqrt{k^{2} / \beta^{2}-\lambda_{n}}} & \text { if } \frac{k^{2}}{\beta^{2}}>\lambda_{n} \\
\frac{i}{2} \frac{1}{\sqrt{k^{2} / \beta^{2}-\lambda_{n}}} & \text { if } \frac{k^{2}}{\beta^{2}}<\lambda_{n}
\end{array} .\right.
$$

In the case of propagating modes $\left(\frac{k^{2}}{\beta^{2}}>\lambda_{n}\right)$ we can differentiate under the integral for $x_{1} \in \mathbb{R} \backslash[-R, R]$ and observe that

$$
\left(\frac{\partial}{\partial\left|x_{1}\right|}-i \sqrt{\frac{k^{2}}{\beta^{2}}-\lambda_{n}}\right) c_{n}\left(x_{1}, y_{1}\right)=0
$$

for every $x_{1} \in \mathbb{R} \backslash[-R, R]$ and $y_{1} \in[-R, R]$. In particular the Sommerfeld radiation condition (first line of (SP2b) is satisfied. For the case of decaying modes we need to show that for all $n \in \mathbb{N}_{0}$ with $\left(\frac{k^{2}}{\beta^{2}}<\lambda_{n}\right)$

$$
\limsup _{\left|x_{1}\right| \rightarrow \infty}\left|b_{u}^{(n)}\left(x_{1}\right)\right|<\infty \text {. }
$$

This is satisfied since for $x_{1}$ sufficiently large, $b_{w_{s}}^{(n)}\left(x_{1}\right)$ decays exponentially. 



\section{3 \\ Chapter}

\section{Inverse Source Problem in Aeroacoustics}

Based on the sound propagation theory from the last chapter, we proceed with a mathematical modelling of the inverse aeroacoustic source problem with correlation data. In particular this includes a formulation of the forward operator on infinite dimensional vector spaces. Essentially we treat the mathematical framework that was presented in HRS20 in some more detail and generality. The main result consists of a uniqueness theorem which is valid for both free-field and waveguide geometries.

3.1. Correlation measurements of random sources . . . . . . . . . . . . . . . . 43

3.2. Uniqueness of the inverse source problem . . . . . . . . . . . . . . 47

\subsection{Correlation measurements of random sources}

This section presents a derivation of the forward operator of the inverse source problem. Some parts are taken from [HRS20, where the forward operator was derived for a free field geometry. As in the last chapter we denote by $D \subset \mathbb{R}^{d}$ with $d \in\{2,3\}$ the open domain of definition (or propagation domain) and we will restrict the analysis to the geometries discussed so far.

Assumption 3.1 (Propagation domain).

Assume that the propagation domain $D \subset \mathbb{R}^{d}$ with $d \in\{2,3\}$ satisfies one of the following

(i) $D=\mathbb{R}^{d}$,

(ii) $D=\mathbb{R} \times \mathcal{A}$, where $\mathcal{A} \subset \mathbb{R}^{d-1}$ satisfies Assumption 2.5 and the eigenfunctions $\psi_{n}$ from Theorem 2.6 satisfy Assumption 2.8.

Moreover we consider the following geometrical setup (cf. [HRS20] and Figure 3.1) for the entire thesis. 


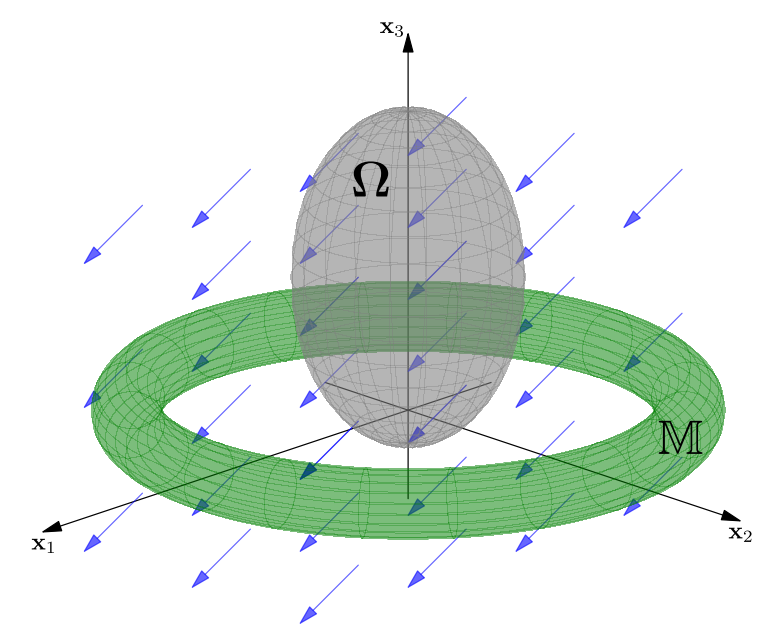

Figure 3.1.: Sketch of a possible geometrical setup in $\mathbb{R}^{3}$.

Assumption 3.2 (Measurement setup).

(i) Assume that $\Omega \subset D$ (the source region) is a bounded, open and connected set such that $D \backslash \bar{\Omega}$ is connected. Furthermore assume there exists an $\epsilon>0$ s.t.

$$
B_{\epsilon}(\Omega):=\left\{\mathbf{x} \in \mathbb{R}^{d} \mid \operatorname{dist}(\Omega, \mathbf{x})<\epsilon\right\} \subset D .
$$

(ii) Assume that $\mathbb{M}$ (the measurement region) is a bounded open set with $\overline{\mathbb{M}} \subset D \backslash \bar{\Omega}$.

Recall the Green's functions for the geometries of interest (cf. 2.19) for $D=\mathbb{R}^{3}$, (2.20) for $D=\mathbb{R}^{2}$ and (2.37) for waveguides)

$$
\begin{aligned}
g(\mathbf{x}, \mathbf{y})= & \frac{\exp \left(\frac{i k}{\beta^{2}}\left(-(\mathbf{x}-\mathbf{y}) \cdot \mathbf{m}+|\mathbf{x}-\mathbf{y}|_{\mathbf{m}}\right)\right)}{4 \pi|\mathbf{x}-\mathbf{y}|_{\mathbf{m}}} \quad(d=3) \\
g(\mathbf{x}, \mathbf{y}) & =\frac{i}{4 \beta} \exp \left(-\frac{i k}{\beta^{2}}(\mathbf{x}-\mathbf{y}) \cdot \mathbf{m}\right) H_{0}^{(1)}\left(\frac{k}{\beta^{2}}|\mathbf{x}-\mathbf{y}|_{\mathbf{m}}\right) \quad(d=2) \\
g_{\mathbf{m}}\left(x_{1}, x_{\perp}, y_{1}, y_{\perp}\right)= & \frac{-i}{2} \exp \left(\frac{-i k|\mathbf{m}|}{\beta^{2}}\left(x_{1}-y_{1}\right)\right) \sum_{n=0}^{n_{\text {prop }}} \frac{1}{s_{n}} \exp \left(i \frac{s_{n}}{\beta^{2}}\left|x_{1}-y_{1}\right|\right) \psi_{n}\left(y_{\perp}\right) \psi_{n}\left(x_{\perp}\right) \\
& -\frac{1}{2} \exp \left(\frac{-i k|\mathbf{m}|}{\beta^{2}}\left(x_{1}-y_{1}\right)\right) \sum_{n=n_{\text {prop }}+1}^{\infty} \frac{1}{s_{n}} \exp \left(-\frac{s_{n}}{\beta^{2}}\left|x_{1}-y_{1}\right|\right) \psi_{n}\left(y_{\perp}\right) \psi_{n}\left(x_{\perp}\right) .
\end{aligned}
$$

The volume potential operator $\mathcal{G}: L^{2}(\Omega) \rightarrow L^{2}(\mathbb{M})$

$$
(\mathcal{G} v)(\mathbf{x}):=\int_{\Omega} g(\mathbf{x}, \mathbf{y}) v(\mathbf{y}) d \mathbf{y}
$$

maps source signals in the source region to measurement signals in the measurement 
region. Note that $\mathcal{G}$ has the adjoint operator $\mathcal{G}^{*}: L^{2}(\mathbb{M}) \rightarrow L^{2}(\Omega)$

$$
\left(\mathcal{G}^{*} w\right)(\mathbf{y})=\int_{\mathbb{M}} \overline{g(\mathbf{x}, \mathbf{y})} w(\mathbf{x}) d \mathbf{x}
$$

For deterministic sources $s \in L^{2}(\Omega)$ and given pressure data $p \in L^{2}(\mathbb{M})$, the inverse source problem then reads as

$$
\text { find } s \in L^{2}(\Omega) \text { s.t. } \mathcal{G} s=p \text {. }
$$

It is well-known that solutions to problem (3.1) are not unique since $\mathcal{G}$ has a nontrivial kernel. This is due to the fact that there exist non-radiating sources i.e. sources $s \in L^{2}(\Omega)$ such that $\mathcal{G} s$ vanishes everywhere outside of $\Omega$. The typical example of such a non-radiating source is

$$
s(\mathbf{y})=L_{k} \phi(\mathbf{y}), \quad \phi \in C_{c}^{\infty}(\Omega) .
$$

Similar to the proof of the fundamental solution property (Propositions 2.13 and 2.17) we get that $L_{k}^{\top} g(\mathbf{x}, \cdot)=\delta_{\mathbf{x}}$ in the sense of distributions. Hence, for a source of type 3.2 and for every $\mathbf{x} \in D \backslash \bar{\Omega}$ we get

$$
(\mathcal{G} s)(\mathbf{x})=\int_{\Omega} g(\mathbf{x}, \mathbf{y}) L_{k} \phi(\mathbf{y}) d \mathbf{y}=0 .
$$

For further reading on the deterministic inverse source problem, we refer to the publications by Griesmaier et al. [GHR12, GHR13, GS16, GS17a, GS17b] and the references therein. However, since aeroacoustic experiments are usually conducted in environments subject to random processes, we will consider a statistical model for source and measurement signals. The aeroacoustic source $Q$ is considered as a Hilbert space process i.e. $Q$ is a continuous linear functional

$$
Q: L^{2}(\Omega) \rightarrow L^{2}(\mathfrak{W}, \mathcal{F}, \mathbb{P})
$$

where $(\mathfrak{W}, \mathcal{F}, \mathbb{P})$ denotes a probability space (cf. [Fra70, LPS89]). This definition covers in particular random variables $Z$ in a Hilbert space $\mathcal{H}$ with finite second order moment (i.e. $\left.\mathbb{E}\left(\|Z\|_{\mathcal{H}}^{2}\right)<\infty\right)$ since

$$
\langle\tilde{Z}, \cdot\rangle_{\mathcal{H}}: \quad \mathcal{H} \rightarrow L^{2}(\mathfrak{W}, \mathcal{F}, \mathbb{P})
$$

defines a Hilbert space process on $\mathcal{H}$. Note that Hilbert space processes are often used to model stochastic errors in an infinite dimensional setup. In that case, a white noise process on a $L^{2}$-space can be interpreted as a random variable in a Sobolev space with negative exponent (see e.g. [WH19, Section 3]).

We recall some basic notions and properties for the source process $Q$ (cf. [BHMR07]). 
Definition \& Proposition 3.3 (Source process).

1. There exists a unique element $\mathbb{E}[Q] \in L^{2}(\Omega)$ (the expectation of $Q$ ) satisfying

$$
\langle\mathbb{E}[Q], v\rangle_{L^{2}(\Omega)}=\mathbb{E}(Q v), \quad \text { for all } v \in L^{2}(\Omega) .
$$

2. There exists a unique linear, continuous operator $\operatorname{Cov}[Q]: L^{2}(\Omega) \rightarrow L^{2}(\Omega)$ (the covariance operator of $Q$ ) satisfying

$$
\left\langle\operatorname{Cov}[Q] v_{1}, v_{2}\right\rangle_{L^{2}(\Omega)}=\operatorname{Cov}\left(Q v_{1}, Q v_{2}\right), \quad \text { for all } v_{1}, v_{2} \in L^{2}(\Omega) .
$$

Moreover, $\operatorname{Cov}[Q]$ is positive semi-definite and self-adjoint.

Aeroacoustic source reconstruction methods often assume that the source is spatially uncorrelated. Therefore the analysis of the inverse problem within this thesis is restricted to uncorrelated sources with zero mean.

Assumption 3.4 (Source signal).

Assume that the source process $Q$ has the following properties

1. $\mathbb{E}[Q]=0$.

2. The source is spatially uncorrelated i.e. there exists a $q \in L^{2}(\Omega)$ such that

$$
\operatorname{Cov}[Q]=M_{q},
$$

where $\left(M_{q} v\right)(\mathbf{x}):=q(\mathbf{x}) v(\mathbf{x})$ denotes the source power multiplication operator.

Note that the source power function $q$ must be bounded (i.e. $q \in L^{\infty}(\Omega)$ ), real-valued and non-negative due to the properties of the covariance operator $\operatorname{Cov}[Q]$. For a fixed position $\mathbf{x} \in \mathbb{M}$ in the measurement region, the observable random pressure signal is given by

$$
p(\mathbf{x})=Q(g(\mathbf{x}, \cdot)) .
$$

Note that $p(\mathbf{x})$ is a complex scalar random variable with zero mean due to Assumption 3.4. The observable correlation data for two positions $\mathbf{x}_{1}, \mathbf{x}_{2} \in \mathbb{M}$ is given by

$$
\begin{aligned}
c_{q}\left(\mathbf{x}_{1}, \mathbf{x}_{2}\right) & :=\operatorname{Cov}\left(p\left(\mathbf{x}_{1}\right), p\left(\mathbf{x}_{2}\right)\right)=\left\langle M_{q} g\left(\mathbf{x}_{1}, \cdot\right), g\left(\mathbf{x}_{2}, \cdot\right)\right\rangle_{L^{2}(\Omega)} \\
& =\int_{\Omega} g\left(\mathbf{x}_{1}, \mathbf{y}\right) \overline{g\left(\mathbf{x}_{2}, \mathbf{y}\right)} q(\mathbf{y}) d \mathbf{y} .
\end{aligned}
$$

By means of the covariance function (3.3), the covariance operator of the random pressure signal $p$ is given by the integral operator $\mathcal{C}(q): L^{2}(\mathbb{M}) \rightarrow L^{2}(\mathbb{M})$

$$
(\mathcal{C}(q) v)\left(\mathbf{x}_{1}\right)=\int_{\mathbb{M}} c_{q}\left(\mathbf{x}_{1}, \mathbf{x}_{2}\right) v\left(\mathbf{x}_{2}\right) d \mathbf{x}_{2}
$$


and can be equivalently represented by

$$
\mathcal{C}(q)=\mathcal{G} M_{q} \mathcal{G}^{*}
$$

The representation of the forward operator by its factorization 3.4 will be of great use for the further analysis of the problem. The next proposition shows that $\mathcal{C}(q) \in$ $\mathcal{L}\left(L^{2}(\mathbb{M})\right)$ is even a Hilbert-Schmidt operator.

Proposition 3.5 (Hilbert-Schmidt covariance operator).

For any $q \in L^{\infty}(\Omega)$, the covariance operator $\mathcal{C}(q)$ belongs to the space of Hilbert-Schmidt operators $\operatorname{HS}\left(L^{2}(\mathbb{M})\right)$.

Proof. Since the Hilbert-Schmidt norm is given by the $L^{2}$ norm of the integral kernel $c_{q}$ (see Proposition B.3), the claim is equivalent to $\left\|c_{q}\right\|_{L^{2}(\mathbb{M} \times \mathbb{M})}<\infty$. As

$$
\left\|c_{q}\right\|_{L^{2}(\mathbb{M} \times \mathbb{M})}^{2} \leq\|q\|_{L^{\infty}(\Omega)}\left(\int_{\mathbb{M}} \int_{\Omega}\left|g\left(\mathbf{x}_{1}, \mathbf{y}\right)\right|^{2} d \mathbf{y} d \mathbf{x}_{1}\right)\left(\int_{\mathbb{M}} \int_{\Omega}\left|g\left(\mathbf{x}_{2}, \mathbf{y}\right)\right|^{2} d \mathbf{y} d \mathbf{x}_{2}\right)
$$

the claim follows from the fact that $g$ is a Hilbert-Schmidt kernel (Proposition 2.12 and 2.15 .

\subsection{Uniqueness of the inverse source problem}

Following the illustrations of the previous section, we define the operator-valued forward operator of the inverse source problem by

$$
\mathcal{C}: L^{\infty}(\Omega) \rightarrow \operatorname{HS}\left(L^{2}(\mathbb{M})\right), \quad q \mapsto \mathcal{C}(q)=\mathcal{G} M_{q} \mathcal{G}^{*},
$$

which is linear and bounded. The following analysis aims at a uniqueness result for the operator equation

$$
\mathcal{C}(q)=C
$$

with $C \in \operatorname{HS}\left(L^{2}(\mathbb{M})\right)$ and the geometrical scenarios described at the beginning of the last section (cf. Assumption 3.1 and Assumption 3.2. We recall the following definitions and calculation rules from Chapter 2

- The volume potential $w_{v}$ of a function $v \in L^{2}(D)$ with compact support is defined by

$$
w_{v}(\mathbf{x})=\int_{D} g(\mathbf{x}, \mathbf{y}) v(\mathbf{y}) d \mathbf{y} \quad \text { for } \mathbf{x} \in D
$$

- With the operators $S: C_{c}^{\infty}(\bar{D}) \subset L^{2}(D) \rightarrow L_{\text {loc }}^{2}(D)$

$$
(S \phi)(\mathbf{y})=\int_{D} g(\mathbf{x}, \mathbf{y}) \phi(\mathbf{x}) d \mathbf{x} \quad \text { for } \mathbf{y} \in D
$$


and $S^{\top}: L_{c}^{2}(D) \subset L^{2}(D) \rightarrow L_{\mathrm{loc}}^{2}(D)$ (cf. Proposition and Definition 2.18 we have for every $u \in L_{c}^{2}(D)$ and $v \in L_{c}^{2}(D) \cap L^{\infty}(D)$

$$
\left\langle S^{\top} u, v\right\rangle_{L^{2}(D)}=\langle u, \bar{S} \bar{v}\rangle_{L^{2}(D)} .
$$

- With the differential operator $L_{k}=k^{2}+2 i k \mathbf{m} \frac{\partial}{\partial x_{1}}-|\mathbf{m}|^{2} \frac{\partial^{2}}{\partial x_{1}^{2}}+\Delta$ we have for any $\phi \in C_{c}^{\infty}(\bar{D})$

$$
\begin{aligned}
L_{k}^{*} & =\overline{L_{k},} \\
L_{k}^{\top}(\phi) & =\overline{L_{k}^{*}(\bar{\phi})} .
\end{aligned}
$$

- For any $v \in L_{c}^{2}(D)$, the volume potential $w_{v}$ satisfies

$$
w_{v}=S^{\top} v
$$

- For any $\phi \in C_{c}^{\infty}(D)$ we have (cf. 2.54) and 2.55)

$$
S\left(L_{k}^{\top} \phi\right)=-\phi \text {. }
$$

For the standard Helmholtz equation in free space it is well known that the volume potential $w_{v}$ is a $C^{\infty}$-solution on the complement of $\operatorname{supp}(v)$. By the existence Theorems for the deterministic source problem (Theorem 2.19 and Theorem 2.20), we get a generalized version of this statement that is valid in the presence of subsonic uniform convection and for the geometries considered here.

Corollary 3.6 (Volume potential solves convected Helmholtz equation). The volume potential of any function $v \in L^{2}(\Omega)$

$$
w_{v}(\mathbf{x})=\int_{\Omega} g(\mathbf{x}, \mathbf{y}) v(\mathbf{y}) d \mathbf{y}
$$

satisfies $w_{v} \in C^{\infty}(D \backslash \bar{\Omega})$ and

$$
L_{k} w_{v}=0 \quad \text { on } D \backslash \bar{\Omega} .
$$

Proof. We note that $U:=D \backslash \bar{\Omega}$ is an open and connected domain in $\mathbb{R}^{d}$. Furthermore, we extend $v$ by zero on whole $D$ (denoting the extension again by $v$ ). By the existence theorems for the deterministic direct source problem (Theorem 2.19 and Theorem 2.20) $w_{v}$ satisfies

$$
\int_{D} w_{v}(\mathbf{x}) L_{k}^{\top} \phi(\mathbf{x}) d \mathbf{x}=-\int_{D} v(\mathbf{x}) \phi(\mathbf{x})
$$

for all test functions $\phi$ (cf. (SP1a) and SP2a). By taking only test functions $\phi \in C_{c}^{\infty}(U)$ 
we get

$$
\int_{U} w_{v}(\mathbf{x}) L_{k}^{\top} \phi(\mathbf{x}) d \mathbf{x}=0 \quad \text { for all } \phi \in C_{c}^{\infty}(U),
$$

since $\operatorname{supp}(v) \cap \operatorname{supp}(\phi)=\emptyset$. Hence $w_{v}$ solves the homogeneous convected Helmholtz equation in $U$ in the sense of distributions. By the regularity result for elliptic differential operators [Fol95, Theorem 6.33 p. 214] we obtain $w_{v} \in C^{\infty}(U)$. Hence

$$
L_{k} w_{v}=0 \text { in } U
$$

in the classical sense.

The next proposition provides an analyticity theorem, similar to [CK13, Theorem 2.2] and [CC06, Theorem 3.2] (solutions of the homogeneous Helmholtz equation are real analytic). For a definition of a real analytic function see Definition B.4.

Proposition 3.7 (Analyticity of $C^{2}$ solutions of the convected Helmholtz equation). Let $U \subset \mathbb{R}^{d}$ be a connected domain and $w \in C^{2}(U)$ such that

$$
L_{k} w=0 \quad \text { on } U \text {. }
$$

Then $w$ is real analytic.

Proof. Let $w \in C^{2}(U)$ be a solution of the homogeneous convected Helmholtz equation at wavenumber $k$ i.e

$$
\left(L_{k} w\right)(\mathbf{x})=\left[k^{2}+2|\mathbf{m}| i k \frac{\partial}{\partial x_{1}}-|\mathbf{m}|^{2} \frac{\partial^{2}}{\partial x_{1}^{2}}+\Delta\right] w(\mathbf{x})=0 \text { in } U
$$

Let $\gamma=\frac{|\mathbf{m}| i k}{\beta}$ and $\mathbf{T}=\operatorname{diag}\left(\frac{1}{\beta}, 1, \ldots\right)^{\top}$ the transformation matrix of the Lorentz transformation. We consider the auxiliary function $\varphi \in C^{2}(\mathbf{T}(U))$ defined by

$$
\varphi(\mathbf{x})=\exp \left(\gamma x_{1}\right) w\left(\mathbf{T}^{-1} \mathbf{x}\right)
$$

By the backward Lorentz transformation (Proposition 2.2) we have that

$$
\left[\frac{k^{2}}{\beta^{2}}+\Delta\right] \varphi(\mathbf{x})=0 \quad \text { in } \mathbf{T}(U)
$$

i.e. $\varphi$ solves the homogeneous standard Helmholtz equation at wavenumber $\frac{k}{\beta}$. By the analyticity theorem for the standard Helmholtz equation on $\mathbb{R}^{d}$ ([CK13, Theorem 2.2] for $d=3$ and [CC06, Theorem 3.2] for $\mathrm{d}=2) \varphi$ is real analytic on $\mathbf{T}(U)$ and by definition

$$
w(\mathbf{x})=\exp \left(-\frac{\gamma}{\beta} x_{1}\right) \varphi(\mathbf{T x}) .
$$


Since $\exp \left(-\frac{\gamma}{\beta} x_{1}\right)$ is real analytic on $\mathbb{R}^{d}, 3.12$ yields that $w$ is analytic on $U$.

Combining the last two propositions we get the following corollary.

Corollary 3.8 (Analyticity of the volume potential).

The volume potential $w_{v}$ is real analytic on $D \backslash \bar{\Omega}$.

Proof. By Proposition 3.6 holds

$$
L w_{v}=0 \quad \text { on } D \backslash \bar{\Omega}
$$

and $D \backslash \bar{\Omega}$ is connected by assumption. Hence, $w_{v}$ is analytic on $D \backslash \bar{\Omega}$ by Proposition 3.7

The essential tool for the proof of uniqueness characterizes a subset of the closure of the range of the volume potential operator.

Proposition 3.9 (Subset of $\overline{\operatorname{ran}\left(\mathcal{G}^{*}\right)}$ ).

The following inclusion is valid:

$$
\mathcal{W} \subset \overline{\operatorname{ran}\left(\mathcal{G}^{*}\right)}
$$

with

$$
\mathcal{W}:=\left\{u \in C^{\infty}(\Omega): L_{k}^{*} u=0 \& \exists \phi_{u} \in C_{c}^{\infty}(D) \text { s.t. }\left.\phi_{u}\right|_{\Omega}=u\right\}
$$

Proof. We can equivalently show that $\mathcal{W} \subset \operatorname{ker}(\mathcal{G})^{\perp}$. So let $v \in \operatorname{ker}(\mathcal{G})$ i.e $w_{v}$ vanishes on $\mathbb{M}$. Due to Corollary $3.8 w_{v}$ is analytic and by analytic continuation [Joh82, p. 65] $w_{v}$ vanishes on $D \backslash \bar{\Omega}$. The extension of $v$ by zero on $D \backslash \Omega$ will be again denoted by $v$. Now for $u \in \mathcal{W}$ we obtain

$$
\begin{gathered}
0=\left\langle w_{v}, L_{k}^{*} u\right\rangle_{L^{2}(\Omega)} \stackrel{\sqrt[3.7]{=}}{=}\left\langle S^{\top} v, L_{k}^{*} \phi_{u}\right\rangle_{L^{2}(\Omega)} \stackrel{3.5}{=}\left\langle v, \overline{S\left(\overline{L_{k}^{*} \phi_{u}}\right)}\right\rangle_{L^{2}(D)} \\
\stackrel{3.6}{=}\left\langle v, \overline{S\left(L_{k}^{\top} \overline{\phi_{u}}\right)}\right\rangle_{L^{2}(D)} \stackrel{3.8)}{=}-\left\langle v, \phi_{u}\right\rangle_{L^{2}(D)}=-\langle v, u\rangle_{L^{2}(\Omega)} .
\end{gathered}
$$

Here we have used the facts that $\operatorname{supp}\left(w_{v}\right) \cap \operatorname{supp}\left(L_{k}^{*} \phi_{u}\right)=\emptyset($ first " $=")$ and $\operatorname{supp}(v) \cap$ $\operatorname{supp}\left(\phi_{u}\right) \subset \Omega$ (last " $\left.="\right)$.

Recall the definition of the Mach norm

$$
|\mathbf{z}|_{\mathbf{m}}=\sqrt{(\mathbf{z} \cdot \mathbf{m})^{2}+\beta^{2}|\mathbf{z}|^{2}},
$$

with corresponding unit sphere

$$
\mathbb{S}_{d-1}(\mathbf{m})=\left\{\mathbf{x} \in \mathbb{R}^{d}:|\mathbf{x}|_{\mathbf{m}}=1\right\} .
$$


Further we need the following auxiliary quantities

$$
\begin{aligned}
\mathbf{A} & =\mathbf{m m}^{\top}+\beta^{2} \mathbf{I} \\
u_{\mathbf{m}, \hat{\mathbf{x}}}(\mathbf{y}) & =\exp \left(\frac{i k}{\beta^{2}}(\mathbf{A} \hat{\mathbf{x}}-\mathbf{m}) \cdot \mathbf{y}\right) .
\end{aligned}
$$

It turns out that plane waves of the form (3.14) solve the convected Helmholtz equation if and only if $\hat{\mathbf{x}} \in \mathbb{S}_{d-1}(\mathbf{m})$. As a consequence, we obtain the following corollary.

Corollary 3.10 (Plane wave solutions).

For any $\hat{\mathbf{x}} \in \mathbb{S}_{d-1}(\mathbf{m})$ we have

$$
u_{\mathbf{m}, \hat{\mathbf{x}}} \in \mathcal{W}
$$

Proof. Firstly, we note that due to the geometry assumptions (Assumption 3.2$), B_{\epsilon}(\Omega) \subset$ $D$ is an open cover of $\bar{\Omega}$. Hence there exists a cutoff function $\eta \in C_{c}^{\infty}(D)$ (see e.g. [AF03, Theorem 3.15]) s.t. $\eta=1$ on $\Omega$ and $0 \leq \eta \leq 1$. Thus for any $u \in C^{\infty}(D)$ we can define the localized function $\phi_{u}=\eta u$, where $\phi_{u}$ and $u$ coincide on $\Omega$. Straightforward calculations show that on $\Omega$

$$
L_{k} u_{\mathbf{m}, \hat{\mathbf{x}}}=L_{k}^{*} u_{\mathbf{m}, \hat{\mathbf{x}}}=0 \quad \text { for } \hat{\mathbf{x}} \in \mathbb{S}_{d-1}(\mathbf{m})
$$

Finally we can prove the uniqueness of the inverse source problem, i.e. the injectivity of the forward operator $\mathcal{C}$.

Theorem 3.11 (Uniqueness c.f. [HRS20]).

If $q_{1}, q_{2} \in L^{\infty}(\Omega)$ such that $\mathcal{C}\left(q_{1}\right)=\mathcal{C}\left(q_{2}\right)$, then

$$
q_{1}=q_{2}
$$

Proof. Due to linearity is suffices to show that $\mathcal{C}(q) \equiv 0$ implies $q=0$. So let $q \in L^{\infty}(\Omega)$ such that $\mathcal{C}(q) \equiv 0$. Then

$$
0=\left\langle\mathcal{G} M_{q} \mathcal{G}^{*} v_{1}, v_{2}\right\rangle_{L^{2}(\mathbb{M})}=\left\langle M_{q} \mathcal{G}^{*} v_{1}, \mathcal{G}^{*} v_{2}\right\rangle_{L^{2}(\Omega)}
$$

for all $v_{1}, v_{2} \in L^{2}(\mathbb{M})$ and hence

$$
\left\langle q u_{1}, u_{2}\right\rangle_{L^{2}(\Omega)}=0 \quad \text { for all } u_{1}, u_{2} \in \operatorname{ran}\left(\mathcal{G}^{*}\right) .
$$

Since $q \in L^{\infty}(\Omega)$, we can apply a density argument to show that property 3.15 holds also for elements of $\overline{\operatorname{ran}\left(\mathcal{G}^{*}\right)}$. By Corollary 3.10 we can choose for $u_{1}$ and $u_{2}$ plane waves of the form $u_{\mathbf{m}, \hat{\mathbf{x}}_{1}}, u_{\mathbf{m}, \hat{\mathbf{x}}_{2}}$ with $\hat{\mathbf{x}}_{1}, \hat{\mathbf{x}}_{2} \in \mathbb{S}_{d-1}(\mathbf{m})$. Together with 3.15 this implies

$$
0=\left\langle q u_{\mathbf{m}, \hat{\mathbf{x}}_{1}}, u_{\mathbf{m}, \hat{\mathbf{x}}_{2}}\right\rangle_{L^{2}(\Omega)}=\int_{\Omega} q(\mathbf{y}) \exp \left(\frac{-i k}{\beta^{2}} \mathbf{A}\left(\hat{\mathbf{x}}_{2}-\hat{\mathbf{x}}_{1}\right) \cdot \mathbf{y}\right) d \mathbf{y}
$$


Note that

$$
\left\{\hat{\mathbf{x}}_{2}-\hat{\mathbf{x}}_{1}: \hat{\mathbf{x}}_{1}, \hat{\mathbf{x}}_{2} \in \mathbb{S}_{d-1}(\mathbf{m})\right\}=\left\{\mathbf{x} \in \mathbb{R}^{d}:|\mathbf{x}|_{\mathbf{m}} \leq 2\right\}
$$

contains an open set $O$ with respect to $|\cdot|$ and the set $V=\frac{k}{\beta^{2}} \mathbf{A}(O)$ is also open as A is a homeomorphism on $\mathbb{R}^{d}$. Extending $q$ by zero to the whole space (denoting the extension again by $q$ ) and using (3.16) we obtain

$$
\hat{q}(\boldsymbol{\xi})=\int_{\mathbb{R}^{d}} q(y) e^{-i \boldsymbol{\xi} \cdot \mathbf{y}} d \mathbf{y}=0 \quad \text { for } \boldsymbol{\xi} \in V,
$$

i.e. the Fourier transform of $q$ vanishes on the open set $V$. Since $q$ has compact support, $\hat{q}$ is real analytic [RS75, Section IX.3], and hence it must vanish everywhere by analytic continuation [Joh82, p. 65]. Since the Fourier transform is injective, we obtain that $q=0$.

Remark 3.12 (Uniqueness for arbitrary domains).

Although the proof of uniqueness that was presented here is restricted to free space and infinite waveguides, the principles apply to a much larger class of domains D. The crucial statement of the uniqueness analysis in this thesis is the Hilbert-Schmidt kernel property of the Green's function (i.e. Propositions 2.12 and 2.15). This property formulates therefore a sufficient condition for uniqueness. Whenever the Green's function is square integrable on every $B \times B$ with $B \subset D$ open and bounded, the strategy of the uniqueness proof presented in this thesis can be applied without significant modifications.

Remark 3.13 (Correlated sources).

As discussed in [HRS20, Remark 3.7] uniqueness does not hold anymore if one allows arbitrary covariance structures for the source covariance operator $\operatorname{Cov}[Q]$. Assuming that $\operatorname{Cov}[Q]$ can be represented by a source correlation function $\kappa_{q}$ i.e.

$$
\operatorname{Cov}[Q](v)\left(\mathbf{y}_{1}\right)=\int_{\Omega} \kappa_{q}\left(\mathbf{y}_{1}, \mathbf{y}_{2}\right) v\left(\mathbf{y}_{2}\right) d \mathbf{y}_{2},
$$

one may impose some further restrictions on $\kappa_{q}$. A reasonable constraint would be, for example

$$
\left|\kappa_{q}\left(\mathbf{y}_{1}, \mathbf{y}_{2}\right)\right| \leq b\left(\left|\mathbf{y}_{1}-\mathbf{y}_{2}\right|\right),
$$

with a rapidly decaying (e.g. exponentially) upper bound b. However, the uniqueness analysis of this thesis cannot be directly transferred to these cases. 


\section{Chapter}

\section{Source Power Imaging Methods}

This chapter discusses several aspects regarding imaging of the source power function with experimental data. In Section 4.1 we focus on three important source imaging methods, namely Beamforming, DAMAS (deconvolution approach for the mapping of acoustic sources) and $C M F$ (covariance matrix fitting). For each of these well-known discrete methods we will present a continuous formulation. The task of reconstructing only the support of the source power function is discussed in Section 4.2. It turns out that the principle of factorization methods that are well-known for example in shape identification problems in inverse scattering can be transferred to the inverse source problem with correlation data. Section 4.3 is concerned with the mathematical modelling of the discrete measurement process and measurement noise.

4.1. Continuous formulations of reconstruction methods . . . . . . . . . . . 54

4.1.1. Adjoint of the forward operator . . . . . . . . . . . . . . 54

4.1.2. Towards discrete reconstruction techniques . . . . . . . . . . 56

4.1.3. Covariance matrix fitting (CMF) . . . . . . . . . . . . . 59

4.1.4. Conventional Beamforming . . . . . . . . . . . . . . . 59

4.1.5. DAMAS . . . . . . . . . . . . . . . . . . . . . . . 61

4.1 .6$. Tikhonov regularization . . . . . . . . . . . . . 63

4.2. The factorization method . . . . . . . . . . . . . . 65

4.3. Discrete correlation estimation . . . . . . . . . . . . . . . 71

4.3.1. Maximum likelihood estimation of the covariance matrix . . . . . 71

4.3.2. Discrete noise model . . . . . . . . . . . . . . . . . 73 


\subsection{Continuous formulations of reconstruction methods}

Many results of this section can be found in [HRS20, Section 4]. Again we consider the geometrical setup described at the beginning of Chapter 3 i.e. Assumption 3.1 and Assumption 3.2 .

\subsubsection{Adjoint of the forward operator}

Recall from Proposition 2.12 and Proposition 2.15 that for the considered geometries (i.e. free space and waveguides with uniformly bounded eigenfunctions) and a bounded set $B \subset D$, the auxiliary function

$$
h_{B}(\mathbf{y})=\int_{B}|g(\mathbf{x}, \mathbf{y})|^{2} d \mathbf{x}
$$

belongs to $L^{\infty}(B)$. By means of this statement the domain of the forward operator $\mathcal{C}$ can be extended from $L^{\infty}(\Omega)$ to $L^{2}(\Omega)$.

Proposition 4.1 (Extended forward operator).

If Assumption 3.1 is valid, then for any $q \in L^{2}(\Omega)$, the covariance operator $\mathcal{C}(q)$ belongs to the space of Hilbert-Schmidt operators $\mathrm{HS}\left(L^{2}(\mathbb{M})\right)$ and $\mathcal{C}: L^{2}(\Omega) \rightarrow \operatorname{HS}\left(L^{2}(\mathbb{M})\right)$ is bounded.

Proof. For the associated integral kernel $c_{q}$ of the operator $\mathcal{C}(q)$ we have

$$
\begin{aligned}
\left\|c_{q}\right\|_{L^{2}(\mathbb{M} \times \mathbb{M})}^{2} & =\int_{\mathbb{M}} \int_{\mathbb{M}}\left|\int_{\Omega} g\left(\mathbf{x}_{1}, \mathbf{y}\right) \overline{g\left(\mathbf{x}_{2}, \mathbf{y}\right)} q(\mathbf{y}) d \mathbf{y}\right|^{2} \\
& \leq\|q\|_{L^{2}(\Omega)}^{2} \int_{\Omega}\left(\int_{\mathbb{M}}|g(\mathbf{x}, \mathbf{y})|^{2} d \mathbf{x}\right)^{2} d \mathbf{y} .
\end{aligned}
$$

Now choose a bounded domain $B \subset D$ with $\Omega \cup \mathbb{M} \subset B$. Then the second factor in the last expression is bounded by $|B|\left\|h_{B}\right\|_{L^{\infty}}^{2}$, which is finite by Proposition 2.12 resp. Proposition 2.15.

Due to Proposition 4.1, the forward operator $\mathcal{C}$ maps between Hilbert spaces. The next proposition characterizes its adjoint.

Proposition 4.2 (Adjoint forward operator $\mathcal{C}^{*}$ [HRS20, Proposition 4.1]). If Assumption 3.1 is valid, then the adjoint of the forward operator

$$
\mathcal{C}: L^{2}(\Omega) \rightarrow \operatorname{HS}\left(L^{2}(\mathbb{M})\right), \quad q \mapsto \mathcal{C}(q)
$$

is given by

$$
\mathcal{C}^{*}: \operatorname{HS}\left(L^{2}(\mathbb{M})\right) \rightarrow L^{2}(\Omega), \quad\left(\mathcal{C}^{*} K\right)(\mathbf{y})=\left\langle K, \mathcal{P}_{\mathbf{y}}\right\rangle_{\mathrm{HS}},
$$

for $K \in \operatorname{HS}\left(L^{2}(\mathbb{M})\right)$ and $\mathbf{y} \in \Omega$ with the monopole operator $\mathcal{P}_{\mathbf{y}} \in \operatorname{HS}\left(L^{2}(\mathbb{M})\right)$ defined by

$$
\left(\mathcal{P}_{\mathbf{y}} \varphi\right)\left(\mathbf{x}_{1}\right)=\int_{\mathbb{M}} g\left(\mathbf{x}_{1}, \mathbf{y}\right) \overline{g\left(\mathbf{x}_{2}, \mathbf{y}\right)} \varphi\left(\mathbf{x}_{2}\right) d \mathbf{x}_{2}
$$


for $\varphi \in L^{2}(\mathbb{M})$ and $\mathbf{x}_{1} \in \mathbb{M}$. If $K$ is associated with its integral kernel $k \in L^{2}(\mathbb{M} \times \mathbb{M})$ (cf. Proposition B.2) we have the representation

$$
\left(\mathcal{C}^{*} K\right)(\mathbf{y})=\int_{\mathbb{M}} \int_{\mathbb{M}} k\left(\mathbf{x}_{1}, \mathbf{x}_{2}\right) \overline{g\left(\mathbf{x}_{1}, \mathbf{y}\right)} g\left(\mathbf{x}_{2}, \mathbf{y}\right) d \mathbf{x}_{1} d \mathbf{x}_{2} .
$$

Proof. For $q \in L^{2}(\Omega)$ and $K \in \operatorname{HS}\left(L^{2}(\mathbb{M})\right)$, we need to show that

$$
\int_{\Omega} q(\mathbf{y}) \overline{\left\langle K, \mathcal{P}_{\mathbf{y}}\right\rangle_{\mathrm{HS}}} d \mathbf{y}=\langle\mathcal{C}(q), K\rangle_{\mathrm{HS}}
$$

Note that $\mathcal{P}_{\mathbf{y}}$ is a Hilbert-Schmidt operator on $L^{2}(\mathbb{M})$ with integral kernel $l_{\mathbf{y}}\left(\mathbf{x}_{1}, \mathbf{x}_{2}\right)=$ $g\left(\mathbf{x}_{1}, \mathbf{y}\right) \overline{g\left(\mathbf{x}_{2}, \mathbf{y}\right)}$. We observe furthermore that

$$
\left\|\mathcal{P}_{\mathbf{y}}\right\|_{\mathrm{HS}}=\left\|l_{\mathbf{y}}\right\|_{L^{2}(\mathbb{M} \times \mathbb{M})}
$$

an with a bounded set $B$ such that $\Omega \cup \mathbb{M} \subset B$

$$
\left\|\mathcal{P}_{\mathbf{y}}\right\|_{\mathrm{HS}} \leq h_{B}(\mathbf{y}) \text { with } h_{B}(\mathbf{y})=\int_{B}|g(\mathbf{x}, \mathbf{y})|^{2} d \mathbf{x} .
$$

Moreover, $h_{B} \in L^{\infty}(B)$ by the integrability properties of $g$ (cf. Proposition 2.12 and Proposition 2.15. Now let $\left\{\varphi_{j}\right\}$ be an orthonormal basis of $L^{2}(\mathbb{M})$. Then $\overline{\left\langle K, \mathcal{P}_{\mathbf{y}}\right\rangle_{\mathrm{HS}}}=$ $\sum_{j=1}^{\infty}\left\langle\mathcal{P}_{\mathbf{y}} \varphi_{j}, K \varphi_{j}\right\rangle$ (cf. Proposition B.2. The sequence

$$
f_{n}(\mathbf{y})=\sum_{j=1}^{n}\left\langle\mathcal{P}_{\mathbf{y}} \varphi_{j}, K \varphi_{j}\right\rangle_{L^{2}(\mathbb{M})}
$$

converges pointwise for all $\mathbf{y} \in \Omega$ and has the integrable majorant $h_{B}(\mathbf{y})\|K\|_{\mathrm{HS}}$. Thus we can interchange the integration over $\Omega$ and the infinite sum in 4.1 by the dominated convergence theorem. This yields

$$
\begin{aligned}
\int_{\Omega} q(\mathbf{y}) \overline{\left\langle K, \mathcal{P}_{\mathbf{y}}\right\rangle_{\mathrm{HS}}} d \mathbf{y} & =\sum_{j=1}^{\infty} \int_{\Omega} q(\mathbf{y})\left\langle\mathcal{P}_{\mathbf{y}} \varphi_{j}, K \varphi_{j}\right\rangle_{L^{2}(\mathbb{M})} d \mathbf{y} \\
& =\sum_{j=1}^{\infty} \int_{\Omega} q(\mathbf{y}) \int_{\mathbb{M}}\left(\mathcal{P}_{\mathbf{y}} \varphi_{j}\right)\left(\mathbf{x}_{1}\right) \overline{\left(K \varphi_{j}\right)\left(\mathbf{x}_{1}\right)} d \mathbf{x}_{1} d \mathbf{y} \\
& =\sum_{j=1}^{\infty} \int_{\mathbb{M}} \overline{\left(K \varphi_{j}\right)\left(\mathbf{x}_{1}\right)} \int_{\Omega} q(\mathbf{y})\left(\mathcal{P}_{\mathbf{y}} \varphi_{j}\right)\left(\mathbf{x}_{1}\right) d \mathbf{y} d \mathbf{x}_{1} \\
& =\sum_{j=1}^{\infty} \int_{\mathbb{M}} \overline{\left(K \varphi_{j}\right)\left(\mathbf{x}_{1}\right)}\left(\mathcal{C}(q) \varphi_{j}\right)\left(\mathbf{x}_{1}\right) d \mathbf{x}_{1}=\langle\mathcal{C}(q), K\rangle_{\mathrm{HS}}
\end{aligned}
$$

where we have used the Fubini-Tonelli theorem (Theorem B.1) in order to interchange the integration over $\Omega$ and $\mathbb{M}$.

The second representation follows by the isometry between $\operatorname{HS}\left(L^{2}(\mathbb{M})\right)$ and $L^{2}(\mathbb{M} \times \mathbb{M})$ 
(see Proposition B.2) since

$$
\left\langle K, \mathcal{P}_{\mathbf{y}}\right\rangle_{\mathrm{HS}\left(L^{2}(\mathbb{M})\right)}=\left\langle k, l_{\mathbf{y}}\right\rangle_{L^{2}(\mathbb{M} \times \mathbb{M})}
$$

Under the assumptions of Proposition 4.1 the extended forward operator is also compact.

Proposition 4.3 (Compact forward operator).

Let Assumption 3.1 be valid. Then the forward operator $\mathcal{C}: L^{2}(\Omega) \rightarrow \operatorname{HS}\left(L^{2}(\mathbb{M})\right)$ is compact.

Proof. We can associate the Hilbert space $\operatorname{HS}\left(L^{2}(\mathbb{M})\right)$ with $L^{2}(\mathbb{M} \times \mathbb{M})$ in the sense of the isometric isomorphism described in Proposition B.3. In that setting $\mathcal{C}$ is an integral operator with integral kernel

$$
\kappa\left(\mathbf{x}_{1}, \mathbf{x}_{2}, \mathbf{y}\right)=g\left(\mathbf{x}_{1}, \mathbf{y}\right) \overline{g\left(\mathbf{x}_{2}, \mathbf{y}\right)} .
$$

Moreover we have $\kappa \in L^{2}([\mathbb{M} \times \mathbb{M}], \Omega)$ since with a bounded set $B$ such that $\Omega \cup \mathbb{M} \subset B$

$$
\int_{\Omega} \int_{\mathbb{M} \times \mathbb{M}}\left|\kappa\left(\mathbf{x}_{1}, \mathbf{x}_{2}, \mathbf{y}\right)\right|^{2} d \mathbf{x}_{1} d \mathbf{x}_{2} d \mathbf{y} \leq \int_{B} h_{B}(\mathbf{y})^{2} d \mathbf{y}<\infty .
$$

Hence, by the characterization of Hilbert-Schmidt operators on $L^{2}$ spaces (see Proposition B.3), $\mathcal{C}$ belongs to $\mathrm{HS}\left(L^{2}(\Omega), L^{2}(\mathbb{M} \times \mathbb{M})\right)$. As Hilbert-Schmidt operators are always compact, this completes the proof. Alternatively one may prove the compactness by means of finite rank approximations of the forward operator (see e.g. DES20, Lemma 2.1]).

\subsubsection{Towards discrete reconstruction techniques}

So far we considered only an infinite dimensional modelling. However, for practical applications we need to consider finite dimensional discretizations. In the engineering literature, propagation and reconstruction are mostly formulated in such a discrete setup since the microphone array consists only of finitely many microphones. The aim of the remainder of this section is to link such finite dimensional formulations to the infinite dimensional setup introduced so far. We focus on three particular (discrete) source reconstruction methods namely Covariance Matrix Fitting (CMF) (also known as spectral estimation method) [BE04, YLSC08], Conventional Beamforming (CBF) [VB88, JD93] and DAMAS [BH06]. For each method we will provide a finite and infinite dimensional formulation. Discrete quantities that have a infinite dimensional counterpart, will be marked by an underscore.

Assume that the microphone array consists of $n_{\text {mic }}$ microphones at positions $\left\{\mathbf{x}_{m}\right\}_{m=1}^{n_{\text {mic }}} \subset$ $\mathbb{M}$. Let further $\left\{\mathfrak{P}_{N}\right\}_{N=1}^{\infty}$ denote a family of partitions of $\Omega$ i.e. for any $N \in \mathbb{N}$ we have 
$\mathfrak{P}_{N}=\left\{\Omega_{1}, \ldots, \Omega_{N}\right\}$ with open sets $\Omega_{n}$ such that

$$
\bar{\Omega}=\bigcup_{n=1}^{N} \overline{\Omega_{n}} \quad \text { and } \quad \Omega_{n} \cap \Omega_{n^{\prime}}=\emptyset \text { if } n \neq n^{\prime} .
$$

We assume further that there exists a continuous, monotonically decreasing function $d$ such that

$$
d(N) \rightarrow 0 \quad \text { as } N \rightarrow \infty \quad \text { and } \quad \max _{\Omega_{n} \in \mathfrak{P}_{N}} \operatorname{diam}\left(\Omega_{n}\right) \leq d(N) \quad \forall N \in \mathbb{N} .
$$

Here diam $\left(\Omega_{n}\right)$ denotes the diameter of the smallest closed ball that completely contains $\Omega_{n}$. For each subdomain $\Omega_{n}$ we fix a corresponding focus point $\mathbf{y}_{n} \in \Omega_{n}$. Consider the orthonormal basis

$$
\phi_{n}=\frac{1}{\sqrt{\left|\Omega_{n}\right|}} 1_{\Omega_{n}} \quad \text { with } \quad X_{N}:=\operatorname{span}\left(\phi_{1}, \ldots, \phi_{N}\right) .
$$

Using this basis, an approximation of the random source $Q$ is given by

$$
\underline{Q}=\sum_{n=1}^{N} \pi_{n} \phi_{n}
$$

where the complex random amplitudes $\pi_{n}$ satisfy

$$
\mathbb{E}\left(\pi_{n}\right)=0 \text { for } n=1, \ldots, N \quad \text { and } \quad \operatorname{Cov}\left(\pi_{n}, \pi_{n^{\prime}}\right)=0 \text { if } n \neq n^{\prime} .
$$

Furthermore we assume that the autopowers are given by the mean value of the source power function on the subdomain i.e.

$$
\mathbb{E}\left(\left|\pi_{n}\right|^{2}\right)=\frac{q_{n}}{\left|\Omega_{n}\right|} \quad \text { with } \quad q_{n}:=\int_{\Omega_{n}} q(\mathbf{y}) d \mathbf{y} .
$$

The next proposition shows that for a continuous source power function and under the previous assumptions, the discrete pressure correlations converge to the true pressure correlations.

Proposition 4.4 (Convergence of discrete source model). Let $\phi_{n}=1_{\Omega_{n}}\left|\Omega_{n}\right|^{-1 / 2}$ and $q \in C^{0}(\bar{\Omega})$ s.t.

$$
\operatorname{Cov}\left(\pi_{n}, \pi_{n^{\prime}}\right)= \begin{cases}0 & \text { if } n \neq n^{\prime} \\ \frac{1}{\left|\Omega_{n}\right|} \int_{\Omega_{n}} q(\mathbf{y}) d \mathbf{y} & \text { if } n=n^{\prime}\end{cases}
$$

and

$$
\underline{Q}=\sum_{n=1}^{N} \pi_{n} \phi_{n}
$$


Then for all $\mathbf{x}_{m}, \mathbf{x}_{l} \in \mathbb{M}$ we have

$$
\operatorname{Cov}\left(\int_{\Omega} g\left(\mathbf{x}_{m}, \mathbf{y}\right) \underline{Q}(\mathbf{y}) d \mathbf{y}, \int_{\Omega} g\left(\mathbf{x}_{l}, \mathbf{y}\right) \underline{Q}(\mathbf{y}) d \mathbf{y}\right) \rightarrow c_{q}\left(\mathbf{x}_{m}, \mathbf{x}_{l}\right) \quad \text { as } N \rightarrow \infty .
$$

Proof. See Appendix A.

For the volume potential operator we obtain the following approximation on the discrete source space $X_{N}(4.2)$

$$
\left(\mathcal{G} \phi_{n}\right)\left(\mathbf{x}_{m}\right)=\frac{1}{\sqrt{\left|\Omega_{n}\right|}} \int_{\Omega_{n}} g\left(\mathbf{x}_{m}, \mathbf{y}\right) d \mathbf{y} \approx \sqrt{\left|\Omega_{n}\right|} \underline{\mathcal{G}}_{m n} \quad \text { with } \quad \underline{\mathcal{G}}_{m n}:=g\left(\mathbf{x}_{m}, \mathbf{y}_{n}\right) .
$$

With $\underline{M_{q}}=\operatorname{diag}\left(q_{1}, \ldots, q_{N}\right)$ this yields the discrete forward operator

$$
\underline{\mathcal{C}}(\underline{q})=\underline{\mathcal{G}} \underline{M q}_{\underline{\mathcal{G}}} \underline{\mathcal{G}}^{*}
$$

Remark 4.5 (Convention for discrete operators).

In [HRS20] the discrete volume potential operator and the discrete source multiplication operator are defined slightly different, namely

$$
\underline{\mathcal{G}}_{m n}=\sqrt{\left|\Omega_{n}\right|} g\left(\mathbf{x}_{m}, \mathbf{y}_{n}\right) \quad \text { and } \quad \underline{M_{q}}=\operatorname{diag}\left(q\left(\mathbf{y}_{1}\right), \ldots, q\left(\mathbf{y}_{N}\right)\right) .
$$

The definitions have been adjusted in this work to be in line with the relevant engineering literature. There it is typically assumed that the random source is composed of a sum of monopoles i.e.

$$
\underline{Q}=\sum_{n=1}^{N} \pi_{n} \delta_{\mathbf{y}_{n}},
$$

where $\delta_{\mathbf{y}_{n}}$ denotes the Dirac distribution at $\mathbf{y}_{n}$. Inserting this assumption yields the pointwise discretization of the volume potental operator $\underline{\mathcal{G}}_{m n}=g\left(\mathbf{x}_{m}, \mathbf{y}_{n}\right)$, random signals $p\left(\mathbf{x}_{m}\right)=\sum_{n=1}^{N} g\left(\mathbf{x}_{m}, \mathbf{y}_{n}\right) \pi_{n}$ and a source power distribution $q=\sum_{n=1}^{N} \mathbb{E}\left(\left|\pi_{n}\right|^{2}\right) \delta_{\mathbf{y}_{n}}$. Using the latter quantities, we have formally

$$
\mathbb{E}\left(\left|\pi_{n}\right|^{2}\right)=\int_{\Omega_{n}} q(\mathbf{y}) d \mathbf{y} .
$$

For a given frequency $\omega$, denote by $p\left(\mathbf{x}_{m}, \omega\right)$ the random pressure signal at the $m$-th microphone. The exact discrete correlation data is given by the cross spectral matrix (CSM) $\underline{C} \in \mathbb{C}^{M \times M}$ with entries

$$
\underline{C}_{m l}=\mathbb{E}\left(p\left(\mathbf{x}_{m}, \omega\right) \overline{p\left(\mathbf{x}_{l}, \omega\right)}\right) .
$$

From now on we will omit the dependency on the frequency $\omega$. Denote by $C^{\text {obs }}$ resp. $\underline{C}^{\text {obs }}$ an empirical estimate of the covariance operator $\mathcal{C}(q)$ (continuous case) resp. the 
cross spectral matrix (discrete case). Since all presented methods were originally formulated for a finite dimensional setup we start with the discrete formulation, followed by an infinite dimensional version.

\subsubsection{Covariance matrix fitting (CMF)}

The discrete version of CMF a.k.a. spectral estimation method (SEM) [BE04, YLSC08] is defined by the least squares problem

$$
\left\|\underline{\mathcal{C}}(\underline{q})-\underline{C}^{\mathrm{obs}}\right\|_{F}^{2}=\min !
$$

where $\|\cdot\|_{F}$ denotes the Frobenius norm. Note that the Frobenius norm is the HilbertSchmidt norm of a finite dimensional matrix. For infinite dimensional quantities the CMF problem reads as

$$
\left\|\mathcal{C}(q)-C^{\mathrm{obs}}\right\|_{\mathrm{HS}}^{2}=\min !
$$

which is uniquely solvable for $q \in L^{\infty}(\Omega)$ and correlation data $C^{\text {obs }} \in \operatorname{ran}\left(\left.\mathcal{C}\right|_{L^{\infty}(\Omega)}\right)$ by Theorem 3.11 .

\subsubsection{Conventional Beamforming}

Conventional Beamforming (CBF) [VB88, JD93 is a widely used source imaging method. It provides a fast and robust estimator of the source power. CBF is defined via an imaging functional that maps each focus point $\mathbf{y} \in \Omega$ to an estimator of the source power $\mathcal{I}(\mathbf{y})$. Since the point evaluations of the imaging functional are independent from each other, no system of equations needs to be solved.

Remark 4.6 (Time domain Beamforming).

Conventional Beamforming in frequency domain is closely related to backpropagation methods in time domain, such as delay-and-sum Beamforming (see e.g. [JD93]) or Kirchhoff migration (see e.g. [BPT05]). The principle of such methods is as follows: For each focus point $\mathbf{y}$ the travel times $\tau\left(\mathbf{x}_{m}, \mathbf{y}\right)$ between the microphone positions and the focus point are computed and all microphone signals are shifted according to $\tau\left(\mathbf{x}_{m}, \mathbf{y}\right)$. Forming a (weighted) sum of the shifted signals causes an amplification of the source signal that originates at $\mathbf{y}$.

The propagation vector $\underline{g}\left(\mathbf{y}_{n}\right) \in \mathbb{C}^{M}$ is defined as the pointwise evaluation of the Green's function at all microphones

$$
\underline{g}\left(\mathbf{y}_{n}\right)=\left(\begin{array}{c}
g\left(\mathbf{x}_{1}, \mathbf{y}_{n}\right) \\
\vdots \\
g\left(\mathbf{x}_{M}, \mathbf{y}_{n}\right)
\end{array}\right) .
$$


The discrete monopole operator $\mathcal{P}_{\mathbf{y}_{n}}=g\left(\mathbf{y}_{n}\right) g\left(\mathbf{y}_{n}\right)^{*} \in \mathbb{C}^{M \times M}$ is therefore also called propagation matrix. In analogy to Proposition 4.2 , the discrete adjoint forward operator is

$$
\underline{\mathcal{C}}^{*}: \mathbb{C}^{M \times M} \rightarrow \mathbb{C}^{N}, \quad\left(\underline{\mathcal{C}}^{*}(\underline{K})\right)_{n}=\left\langle\underline{K}, \underline{\mathcal{P}_{\mathbf{y}_{n}}}\right\rangle_{F},
$$

where $\langle\cdot, \cdot\rangle_{F}$ denotes the Frobenius scalar product. Note that we can equivalently characterize the discrete adjoint forward operator by

$$
\underline{\mathcal{C}}^{*}(\underline{K})=\operatorname{diag}\left(\underline{\mathcal{G}}^{*} \underline{K \mathcal{G}}\right) .
$$

The $\mathrm{CBF}$ functional can be characterized by a one dimensional minimization problem (see e.g. [Sij04])

$$
\underline{\mathcal{I}}\left(\mathbf{y}_{n}\right)=\underset{\mu \in \mathbb{C}}{\operatorname{argmin}}\left\|\underline{C}^{\text {obs }}-\mu \underline{\mathcal{P}_{\mathbf{y}_{n}}}\right\|_{F}^{2}
$$

The solution to 4.6 is given by

$$
\underline{\mathcal{I}}\left(\mathbf{y}_{n}\right)=\frac{g\left(\mathbf{y}_{n}\right)^{*} \underline{C}^{\mathrm{obs}} \underline{g}\left(\mathbf{y}_{n}\right)}{\left|\underline{g}\left(\mathbf{y}_{n}\right)\right|^{4}}=\frac{\left\langle\underline{C}^{\mathrm{obs}}, \underline{\mathcal{P}_{\mathbf{y}_{n}}}\right\rangle_{F}}{\left\|\underline{\mathcal{P}_{\mathbf{y}_{n}}}\right\|_{F}^{2}}=\frac{\left(\underline{\mathcal{C}}^{*}\left(\underline{C}^{\text {obs }}\right)\right)\left(\mathbf{y}_{n}\right)}{\left\|\underline{\mathcal{P}_{\mathbf{y}_{n}}}\right\|_{F}^{2}} .
$$

Note that for $C^{\text {obs }}$ Hermitian, $\underline{I}\left(\mathbf{y}_{n}\right) \in \mathbb{R}$ and for $C^{\text {obs }}$ moreover positive semi-definite, $\underline{\mathcal{I}}\left(\mathbf{y}_{n}\right) \geq 0$.

For the continuous version we obtain analogously the characterizing minimization problem

$$
\mathcal{I}(\mathbf{y}):=\underset{\mu \in \mathbb{C}}{\operatorname{argmin}}\left\|C^{\mathrm{obs}}-\mu \mathcal{P}_{\mathbf{y}}\right\|_{\mathrm{HS}}^{2}
$$

with solution

$$
\mathcal{I}(\mathbf{y})=\frac{\left\langle C^{\mathrm{obs}}, \mathcal{P}_{\mathbf{y}}\right\rangle_{\mathrm{HS}}}{\left\|\mathcal{P}_{\mathbf{y}}\right\|_{\mathrm{HS}}^{2}}=\frac{\left(\mathcal{C}^{*}\left(C^{\mathrm{obs}}\right)\right)(\mathbf{y})}{\left\|\mathcal{P}_{\mathbf{y}}\right\|_{\mathrm{HS}}^{2}}
$$

Again $\mathcal{I}$ maps to the real numbers if $C^{\text {obs }}$ is self-adjoint and maps to the the non-negative real numbers if $C^{\text {obs }}$ is moreover positive semi-definite. The Hilbert-Schmidt norm of the monopole operator is given by

$$
\left\|\mathcal{P}_{\mathbf{y}}\right\|_{\mathrm{HS}}^{2}=\int_{\mathbb{M} \times \mathbb{M}}\left|g\left(\mathbf{x}_{1}, \mathbf{y}\right) \overline{g\left(\mathbf{x}_{2}, \mathbf{y}\right)}\right|^{2} d\left(\mathbf{x}_{1}, \mathbf{x}_{2}\right)=\|g(\cdot, \mathbf{y})\|_{L^{2}(\mathbb{M})}^{4}>0 .
$$

Remark 4.7 (Interpretation of Beamforming results).

Note that Conventional Beamforming is not a quantitative reconstruction method i.e. we cannot expect that the imaging procedure yields the true source power function. Whenever 
$\sup _{\mathbf{y} \in \Omega}\left\|\mathcal{P}_{\mathbf{y}}\right\|_{\mathrm{HS}}^{-1}<\infty$, the mapping

$$
C^{\text {obs }} \mapsto \frac{\left\langle C^{\text {obs }}, \mathcal{P}_{\mathbf{y}}\right\rangle_{\mathrm{HS}}}{\left\|\mathcal{P}_{\mathbf{y}}\right\|_{\mathrm{HS}}^{2}}
$$

defines a linear and bounded operator from $\operatorname{HS}\left(L^{2}(\mathbb{M})\right)$ to $L^{2}(\Omega)$. Therefore, it cannot be the inverse of $\mathcal{C}$ because $\mathcal{C}$ is compact and hence, its inverse must be unbounded. However, as the evaluation of the imaging functional does not require the solution of a large minimization problem, the Conventional Beamforming result can be computed very fast.

\subsubsection{DAMAS}

DAMAS (deconvolution approach for the mapping of acoustic sources) is an inverse method that aims at deblurring the source information of a CBF solution. Again we start with the discrete formulation as it was introduced by Brooks \& Humphreys [BH06], which is given by the following linear system

$$
\underline{\mathcal{I}}\left(\mathbf{y}_{n}\right)=\sum_{n^{\prime}=1}^{N} \underline{\psi}\left(\mathbf{y}_{n}, \mathbf{y}_{n^{\prime}}\right) q_{n^{\prime}}
$$

Here $\underline{\psi}$ is usually referred to as point spread function (PSF) and

$$
\underline{\psi}\left(\mathbf{y}_{n}, \mathbf{y}_{n^{\prime}}\right)=\frac{\left\langle\underline{\mathcal{P}_{\mathbf{y}_{n^{\prime}}},}, \underline{\mathcal{P}_{\mathbf{y}_{n}}}\right\rangle_{F}}{\left\|\underline{\mathcal{P}_{\mathbf{y}_{n}}}\right\|_{F}^{2}} .
$$

The formulation on function spaces is given by an integral equation of the first kind,

$$
\mathcal{I}(\mathbf{y})=\int_{\Omega} \psi\left(\mathbf{y}, \mathbf{y}^{\prime}\right) q\left(\mathbf{y}^{\prime}\right) d \mathbf{y}^{\prime} .
$$

With the PSF

$$
\psi\left(\mathbf{y}, \mathbf{y}^{\prime}\right)=\frac{\left\langle\mathcal{P}_{\mathbf{y}^{\prime}}, \mathcal{P}_{\mathbf{y}}\right\rangle_{\mathrm{HS}}}{\left\|\mathcal{P}_{\mathbf{y}}\right\|_{\mathrm{HS}}^{2}}=\frac{\left(\mathcal{C}^{*}\left(\mathcal{P}_{\mathbf{y}^{\prime}}\right)\right)(\mathbf{y})}{\left\|\mathcal{P}_{\mathbf{y}}\right\|_{\mathrm{HS}}^{2}} .
$$

Remark 4.8 (DAMAS Nomenclature).

For a shift invariant PSF, (4.9) reduces to a convolution integral. However in the scenarios, considered in this thesis the PSF is not shift invariant. Nevertheless, deblurring methods like DAMAS are usually called deconvolution methods in the aeroacoustic community. Furthermore within this theses, DAMAS will always refer to the integral equation (4.9). This is emphasized here since in most literature DAMAS refers to the iterative Gauß-Seidel method, suggested in [BH06] in order to solve the finite dimensional linear problem 4.8.

The integral equation of DAMAS (4.9) and the least squares problem of CMF 4.4 
are closely related, as the next proposition shows.

Proposition 4.9 (Normal equation [HRS20, Proposition 4.3]).

The DAMAS problem (4.9) is equivalent to the operator equation

$$
\mathcal{C}^{*} \mathcal{C}(q)=\mathcal{C}^{*} C^{\text {obs }}
$$

which is the normal equation of the CMF problem (4.4).

Proof. First of all we can multiply (4.9) by $\left\|\mathcal{P}_{\mathbf{y}}\right\|_{\text {HS }}^{2}$ which yields the equivalent integral equation

$$
\left(\mathcal{C}^{*}\left(C^{\mathrm{obs}}\right)\right)(\mathbf{y})=\int_{\Omega}\left\langle\mathcal{P}_{\mathbf{y}^{\prime}}, \mathcal{P}_{\mathbf{y}}\right\rangle_{\mathrm{HS}} q\left(\mathbf{y}^{\prime}\right) d \mathbf{y}^{\prime}
$$

For an orthonormal basis $\left\{\varphi_{j}\right\}_{j \in \mathbb{N}}$ of $L^{2}(\mathbb{M})$, reformulating the right-hand side of 4.11) yields

$$
\begin{aligned}
\int_{\Omega}\left\langle\mathcal{P}_{\mathbf{y}^{\prime}}, \mathcal{P}_{\mathbf{y}}\right\rangle_{\mathrm{HS}} q\left(\mathbf{y}^{\prime}\right) d \mathbf{y}^{\prime} & =\int_{\Omega} \sum_{j=1}^{\infty}\left\langle\mathcal{P}_{\mathbf{y}^{\prime}} \varphi_{j}, \mathcal{P}_{\mathbf{y}} \varphi_{j}\right\rangle_{L^{2}(\mathbb{M})} q\left(\mathbf{y}^{\prime}\right) d \mathbf{y}^{\prime} \\
& =\sum_{j=1}^{\infty} \int_{\Omega}\left\langle\mathcal{P}_{\mathbf{y}^{\prime}} \varphi_{j}, \mathcal{P}_{\mathbf{y}} \varphi_{j}\right\rangle_{L^{2}(\mathbb{M})} q\left(\mathbf{y}^{\prime}\right) d \mathbf{y}^{\prime} \\
& =\sum_{j=1}^{\infty} \int_{\mathbb{M}}\left[\int_{\Omega}\left(\mathcal{P}_{\mathbf{y}^{\prime}} \varphi_{j}\right)(\mathbf{x}) q\left(\mathbf{y}^{\prime}\right) d \mathbf{y}^{\prime}\right] \overline{\left(\mathcal{P}_{\mathbf{y}} \varphi_{j}\right)(\mathbf{x})} d \mathbf{x}
\end{aligned}
$$

We obtain further

$$
\begin{aligned}
{[\ldots] } & =\int_{\Omega}\left(\int_{\mathbb{M}} g\left(\mathbf{x}, \mathbf{y}^{\prime}\right) \overline{g\left(\mathbf{x}^{\prime}, \mathbf{y}^{\prime}\right)} \varphi_{j}\left(\mathbf{x}^{\prime}\right) d \mathbf{x}^{\prime}\right) q\left(\mathbf{y}^{\prime}\right) d \mathbf{y}^{\prime} \\
& =\int_{\mathbb{M}} c_{q}\left(\mathbf{x}, \mathbf{x}^{\prime}\right) \varphi_{j}\left(\mathbf{x}^{\prime}\right) d \mathbf{x}^{\prime}=\left(\mathcal{C}(q) \varphi_{j}\right)(\mathbf{x}) .
\end{aligned}
$$

Inserting 4.13 into 4.12) yields

$$
\int_{\Omega}\left\langle\mathcal{P}_{\mathbf{y}^{\prime}}, \mathcal{P}_{\mathbf{y}}\right\rangle_{\mathrm{HS}} q\left(\mathbf{y}^{\prime}\right) d \mathbf{y}^{\prime}=\left\langle\mathcal{C}(q), \mathcal{P}_{\mathbf{y}}\right\rangle_{\mathrm{HS}}=\left(\mathcal{C}^{*} \mathcal{C}(q)\right)(\mathbf{y})
$$

The relation of DAMAS and CMF is similar for the discrete versions.

Corollary 4.10 (Discrete normal equation [HRS20, Corollary 4.5]).

The problem (4.8) is equivalent to the linear system

$$
\underline{\mathcal{C}}^{*} \underline{\mathcal{C}}(\underline{q})=\underline{\mathcal{C}}^{*} \underline{C}^{\text {obs }}
$$

which is the normal equation of 4.3 .

From the last proposition we can easily deduce a uniqueness result for the DAMAS integral equation. 
Corollary 4.11 (DAMAS uniqueness [HRS20, Corollary 4.4]).

For exact data $C^{\mathrm{obs}}=\mathcal{C}(q)$ with $q \in L^{\infty}(\Omega)$, the solution of 4.9 is unique in $L^{\infty}(\Omega)$.

Proof. By the uniqueness theorem of the inverse problem (Theorem 3.11) we obtain

$$
\operatorname{ker}\left(\mathcal{C}^{*} \mathcal{C}\right) \cap L^{\infty}(\Omega)=\operatorname{ker}(\mathcal{C}) \cap L^{\infty}(\Omega)=\{0\} .
$$

Remark 4.12 (DAMAS and CMF without regularization).

A possible strategy for the solution of the CMF or DAMAS problem may be the selection of a (least squares) solution of minimum norm, often called best-approximate solution. Consider the sets of solutions for given data $C^{\text {obs }} \in \mathrm{HS}\left(L^{2}(\mathbb{M})\right)$ given by

$$
\begin{array}{r}
\operatorname{sol}^{\mathrm{CMF}}\left(C^{\mathrm{obs}}\right)=\underset{q \in L^{2}(\Omega)}{\operatorname{argmin}}\left\|\mathcal{C} q-C^{\mathrm{obs}}\right\|_{\mathrm{HS}\left(L^{2}(\mathbb{M})\right)}, \\
\operatorname{sol}^{\text {DAMAS }}\left(C^{\text {obs }}\right)=\left\{q \in L^{2}(\Omega): \mathcal{C}^{*} \mathcal{C}(q)=\mathcal{C}^{*} C^{\text {obs }}\right\} .
\end{array}
$$

For $C^{\text {obs }} \in \operatorname{ran}(\mathcal{C})+\operatorname{ran}(\mathcal{C})^{\perp}$, we have that $q$ is a least squares solution if and only if the normal equation is satisfied i.e. $\mathrm{sol}^{\mathrm{CMF}}\left(C^{\mathrm{obs}}\right)=\mathrm{sol}^{\mathrm{DAMAS}}\left(C^{\mathrm{obs}}\right)$ (see [EHN96, Section 2.1.]). Moreover in that case, there exists a unique best-approximate solution $q^{\dagger} \in \mathrm{sol}^{\mathrm{CMF}}\left(C^{\mathrm{obs}}\right)$ that satisfies

$$
\left\|q^{\dagger}\right\|_{L^{2}(\Omega)}=\inf \left\{\|\tilde{q}\|_{L^{2}(\Omega)}: \tilde{q} \in \mathrm{sol}^{\mathrm{CMF}}\left(C^{\mathrm{obs}}\right)\right\} .
$$

The best-approximate solution can be characterized by

$$
q^{\dagger}=\mathcal{C}^{\dagger} C^{\text {obs }}
$$

where $\mathcal{C}^{\dagger}$ denotes the Moore-Penrose generalized inverse (see [EHN96, Definition 2.2. and Theorem 2.5.]). As the forward operator $\mathcal{C}$ is compact (Proposition 4.3), $\mathcal{C}^{\dagger}$ is an unbounded linear operator (see [EHN96, Proposition 2.7.]). Hence, small errors in the data can lead to arbitrarily large deviations in the best-approximate solution.

\subsubsection{Tikhonov regularization}

We have seen in Proposition 4.3 that $\mathcal{C}: L^{2}(\Omega) \rightarrow \operatorname{HS}\left(L^{2}(\mathbb{M})\right)$ is compact. Since $\operatorname{ran}(\mathcal{C})$ is infinite dimensional this implies that the inverse source problem

$$
\mathcal{C}(q)=C
$$

is ill-posed [CK13, Theorem 4.2 p.96]. Such problems require regularization to avoid unstable results in case of noisy data (cf. Remark 4.12). As a regularization technique we will consider generalized Tikhonov regularization within this thesis. For a generic convex (cf. Definition B.5 penalty functional $\mathcal{R}$ and a regularization parameter $\alpha>0$ 
this yields source power estimators of the form

$$
\widehat{q}_{C M F, \alpha} \in \underset{q \in L^{2}(\Omega)}{\operatorname{argmin}}\left[\left\|\mathcal{C}(q)-C^{\mathrm{obs}}\right\|_{\mathrm{HS}\left(L^{2}(\mathbb{M})\right)}^{2}+\alpha \mathcal{R}(q)\right]
$$

in case of $\mathrm{CMF}$ and

$$
\widehat{q}_{D A M A S, \alpha} \in \underset{q \in L^{2}(\Omega)}{\operatorname{argmin}}\left[\left\|\mathcal{C}^{*} \mathcal{C}(q)-\mathcal{C}^{*} C^{\text {obs }}\right\|_{L^{2}(\Omega)}^{2}+\alpha \mathcal{R}(q)\right]
$$

in case of DAMAS. Some typical examples of penalty functionals used in acoustic source imaging are (see e.g. [YLSC08, $\mathrm{LPB}^{+} 17$ ])

- $\mathcal{R}(q)=\frac{1}{2}\|q\|_{L^{2}(\Omega)}^{2}$

- $\mathcal{R}(q)=\|q\|_{L^{1}(\Omega)}$

- $\mathcal{R}(q)= \begin{cases}0 & \text { if } q(\mathbf{y}) \in[a, b] \text { for a.e.y } \\ \infty & \text { else }\end{cases}$
( $L^{2}$ norm penalty),

( $L^{1}$ norm penalty)

(box constraint with bounds $a, b \in \mathbb{R}$ ).

To discuss the existence and uniqueness of the Tikhonov minimizers 4.15) and 4.16) we need some further assumptions on the penalty functional $\mathcal{R}$.

Assumption 4.13 (Properties of penalty functional).

Let $\mathcal{R}: L^{2}(\Omega) \rightarrow[0, \infty]$ with $\operatorname{dom}(\mathcal{R}):=\left\{f \in L^{2}(\Omega): \mathcal{R}(f)<\infty\right\} \neq \emptyset$ be weakly sequentially lower semicompact. This explicitly means: for any $\beta \geq 0$ the sublevel sets $\operatorname{lev}_{\beta}(\mathcal{R}):=\left\{f \in L^{2}(\Omega): \mathcal{R}(f) \leq \beta\right\}$ are weakly sequentially compact i.e. for any sequence $\left(f_{n}\right)_{n \in \mathbb{N}} \subset \operatorname{lev}_{\beta}(\mathcal{R})$ there exists a subsequence $\left(f_{n_{k}}\right)_{k \in \mathbb{N}}$ such that $f_{n_{k}}$ converges weakly to some $f \in \operatorname{lev}_{\beta}(\mathcal{R})$.

A sufficient condition for the existence and uniqueness of source power estimators is provided by the following theorem.

Theorem 4.14 (Existence and uniqueness of minimizers).

Assume that $\mathcal{R}$ fulfills Assumption 4.13, then there exist Tikhonov minimizers 4.15) and (4.16). If $\mathcal{R}$ is moreover strictly convex, then the minimizers are unique.

Proof. By assumption the sublevel sets of $\mathcal{R}$ are weakly sequentially compact and therefore in particular weakly sequentially closed. The latter fact is equivalent to $\mathcal{R}$ being weakly sequentially lower semicontinuous (cf. Definition B.6 and [BC17, Lemma 1.36, p.16]). If $\mathcal{R}$ is strictly convex, the entire Tikhonov functional is stricly convex since the data fidelity term $\left\|\mathcal{C}(q)-C^{\text {obs }}\right\|_{\mathrm{HS}\left(L^{2}(\mathbb{M})\right)}^{2}$ resp. $\left\|\mathcal{C}^{*} \mathcal{C}(q)-\mathcal{C}^{*} C^{\text {obs }}\right\|_{L^{2}(\Omega)}^{2}$ is convex. The statement then follows by the theory on minimization of convex Tikhonov functionals (see e.g. [MST12, Theorem 2.5]). 


\subsection{The factorization method 1}

So far we treated the problem of reconstructing the source power function $q$ from correlation data. However, this section is concerned with the localization problem only i.e. the problem of reconstructing the support of the source power function. Such shape identification problems are well-studied for inverse problems e.g. in the field of inverse scattering. Here we will only deal with the so-called factorization method that yields a necessary and sufficient condition that some point $\mathbf{z} \in \mathbb{R}^{d}$ belongs to the shape that should be reconstructed. The factorization method was introduced by Kirsch in the late 1990s with applications to shape identification in inverse obstacle scattering Kir98. and identification of the support of the perturbation of the refractive index in inverse medium scattering [Kir99]. For a deeper analysis of the factorization method along with applications in several fields in inverse problems we refer to the monograph [KG07]. If we apply the principles of the factorization method to the aeroacoustic inverse source problem, we will observe that this leads exactly to the method of Capon Cap69. Surprisingly, in the referenced article, this method was derived by a completely different approach in a discrete setting.

Within this section we restrict the analysis to a free space geometry in two or three spatial dimensions. Hence the Green's function is of the form $(2.19)$ or $(2.20)$. For a given source power function $q \in L^{\infty}(\Omega)$ we define the interior of the essential support

$$
\Omega_{q}:=\operatorname{int}(\operatorname{ess} \operatorname{supp}(q))=\operatorname{int}\left(\mathbb{R}^{d} \backslash \bigcup\left\{O \subset \mathbb{R}^{d}: O \text { open and } q=0 \text { a.e. in } O\right\}\right) .
$$

For $q$ fixed we define the restricted operator $\mathcal{G}_{\Omega_{q}}: L^{2}\left(\Omega_{q}\right) \rightarrow L^{2}(\mathbb{M})$

$$
\left(\mathcal{G}_{\Omega_{q}} v\right)(\mathbf{x}):=\int_{\Omega_{q}} g(\mathbf{x}, \mathbf{y}) v(\mathbf{y}) d \mathbf{y} \quad \text { for } \mathbf{x} \in \mathbb{M}
$$

with adjoint $\mathcal{G}_{\Omega_{q}}^{*}: L^{2}(\mathbb{M}) \rightarrow L^{2}\left(\Omega_{q}\right)$ given by

$$
\left(\mathcal{G}_{\Omega_{q}}^{*} w\right)(\mathbf{y})=\int_{\mathbb{M}} \overline{g(\mathbf{x}, \mathbf{y})} w(\mathbf{x}) d \mathbf{x} \quad \text { for } \mathbf{y} \in \Omega_{q} .
$$

The proofs in this section require some additional assumptions on the source power function $q$.

Assumption 4.15 (Source properties).

1. $\exists q_{0}>0$ such that $q(\mathbf{y}) \geq q_{0}$ for a.e. $\mathbf{y} \in \Omega_{q}$,

2. $\mathbb{R}^{d} \backslash \overline{\Omega_{q}}$ is connected.

\footnotetext{
${ }^{1}$ The content of this section was initiated at the Obwerwolfach workshop "computational inverse problems for partial differential equations" Mat20
} 
The following theorem yields a criterion to identify $\Omega_{q}$ by knowing only the covariance operator $\mathcal{C}(q)$.

Theorem 4.16 (Range criteria).

Let $D=\mathbb{R}^{d}$ i.e. the Green's function is given by 2.19$)$ or $(2.20)$. Furthermore, let $q$ satisfy Assumption 4.15 and let $\mathbf{y}_{0} \in \Omega$. Then the following statements are equivalent.

(i) $g\left(\cdot, \mathbf{y}_{0}\right) \in \operatorname{ran}\left(\mathcal{C}(q)^{1 / 2}\right)$.

(ii) $g\left(\cdot, \mathbf{y}_{0}\right) \in \operatorname{ran}\left(\mathcal{G}_{\Omega_{q}}\right)$.

(iii) $\inf \left\{\left|\langle w, \mathcal{C}(q) w\rangle_{L^{2}(\mathbb{M})}\right|: w \in L^{2}(\mathbb{M}),\left\langle w, g\left(\cdot, \mathbf{y}_{0}\right)\right\rangle_{L^{2}(\mathbb{M})}=1\right\}>0$.

(iv) $\mathbf{y}_{0} \in \Omega_{q}$.

Proof. We start with a few general observations.

- $M_{q}$ is coercive on $L^{2}\left(\Omega_{q}\right)$ i.e. $\forall v \in L^{2}\left(\Omega_{q}\right)$ we have

$$
\left\langle v, M_{q} v\right\rangle_{L^{2}\left(\Omega_{q}\right)} \geq q_{0}\|v\|_{L^{2}\left(\Omega_{q}\right)}^{2} .
$$

- We have the factorization

$$
\mathcal{C}(q)=\mathcal{G}_{\Omega_{q}} M_{q} \mathcal{G}_{\Omega_{q}}^{*}
$$

- $g\left(\cdot, \mathbf{y}_{0}\right) \in L^{2}(\mathbb{M})$ (i.e. all terms are well defined).

$"(i) \Leftrightarrow(i i) ":$

The factorization (4.18) satisfies the assumptions of [KG07, Corollary 1.22]. Thus we have $\operatorname{ran}\left(\mathcal{C}(q)^{1 / 2}\right)=\operatorname{ran}\left(\mathcal{G}_{\Omega_{q}}\right)$. For this specific problem one may easily prove this relation directly as follows: We have the decomposition $\mathcal{C}(q)^{1 / 2}=\left(B B^{*}\right)^{1 / 2}=\left|B^{*}\right|$, where $B=\mathcal{G}_{\Omega_{q}} M_{\sqrt{q}}$ and the absolute value of an operator $A$ is defined as $|A|=\left(A^{*} A\right)^{1 / 2}$. By polar decomposition (see e.g. [Sun97, Theorem 4.2.5, p. 120]) there exists a partial isometry $U$ such that

$$
B=\left|B^{*}\right| U^{*} \quad \text { and } \quad\left|B^{*}\right|=B U .
$$

Now (4.19) implies that $\operatorname{ran}\left(\left|B^{*}\right|\right)=\operatorname{ran}(B)$. Since $M_{\sqrt{q}}$ is an isomorphism on $\Omega_{q}$ we conclude that $\operatorname{ran}(B)=\operatorname{ran}\left(\mathcal{G}_{\Omega_{q}}\right)$ and hence

$$
\operatorname{ran}\left(\mathcal{C}(q)^{1 / 2}\right)=\operatorname{ran}\left(\left|B^{*}\right|\right)=\operatorname{ran}(B)=\operatorname{ran}\left(\mathcal{G}_{\Omega_{q}}\right) .
$$

$"(i i) \Leftrightarrow($ iii $) ":$

It holds more generally by [KG07, Theorem 1.16], that for any $\phi \in L^{2}(\mathbb{M}), \phi \neq 0$ that $\phi \in \operatorname{ran}\left(\mathcal{G}_{\Omega_{q}}\right)$ if and only if the inf-criterion is satisfied. 
$"(i i) \Rightarrow(i v) ":$

Proof by contradiction. Assume there exists a $v_{0} \in L^{2}\left(\Omega_{q}\right)$ such that

$$
g\left(\cdot, \mathbf{y}_{0}\right)=\mathcal{G}_{\Omega_{q}} v_{0}=: w
$$

and that $\mathbf{y}_{0} \notin \Omega_{q}$. By definition of the volume potential operator and analytic continuation, $w$ and $g\left(\cdot, \mathbf{y}_{0}\right)$ can be extended to $\mathbb{R}^{d} \backslash \overline{\Omega_{q}}$ such that

$$
g\left(\mathbf{x}, \mathbf{y}_{0}\right)=w(\mathbf{x}) \quad \text { for } \mathbf{x} \in \mathbb{R}^{d} \backslash \overline{\Omega_{q}} .
$$

Now denote by $B_{1}\left(\mathbf{y}_{0}\right)$ the ball with radius 1 and center $\mathbf{y}_{0}$ and set

$$
K\left(\mathbf{y}_{0}\right):=\left(\mathbb{R}^{d} \backslash \overline{\Omega_{q}}\right) \cap B_{1}\left(\mathbf{y}_{0}\right) .
$$

We obtain further for $\mathbf{x} \in K\left(\mathbf{y}_{0}\right)$ and any bounded set $B$ with $\left(K\left(\mathbf{y}_{0}\right) \cup \Omega_{q}\right) \subset B$

$$
|w(\mathbf{x})| \leq\left\|v_{0}\right\|_{L^{2}\left(\Omega_{q}\right)} \sqrt{\int_{B}|g(\mathbf{x}, \mathbf{y})|^{2} d \mathbf{y}} .
$$

As $|g(\mathbf{x}, \mathbf{y})|=|g(\mathbf{y}, \mathbf{x})|$, we can use the integrability properties of $g$ (cf. Proposition 2.12 and Proposition 2.15 to get an upper bound for the expression under the square root uniformly for all $\mathbf{x} \in K\left(\mathbf{y}_{0}\right)$. This proves that $w \in L^{\infty}\left(K\left(\mathbf{y}_{0}\right)\right)$. On the other hand

$$
\lim _{\mathbf{x} \rightarrow \mathbf{y}_{0}}\left|g\left(\mathbf{x}, \mathbf{y}_{0}\right)\right|=\infty .
$$

This is a contradiction and hence $\mathbf{y}_{0} \in \Omega_{q}$.

$"(i v) \Rightarrow(i i) ":$

We construct an element $v \in L^{2}\left(\Omega_{q}\right)$ such that

$$
\mathcal{G}_{\Omega_{q}} v=g\left(\cdot, \mathbf{y}_{0}\right) \quad \text { in } \mathbb{M} .
$$

Since $\mathbf{y}_{0} \in \Omega_{q}$ by assumption, there exist an $\epsilon>0$ such that the ball $B_{\epsilon}\left(\mathbf{y}_{0}\right)$ is completely contained in $\Omega_{q}$. Now take a function $\eta \in C^{\infty}(\mathbb{R})$ with $0 \leq \eta(s) \leq 1, \eta(s)=0$ for $s<\frac{\epsilon}{2}$ and $\eta(s)=1$ for $s \geq \epsilon$. Then the function

$$
\phi(\mathbf{y}):=\eta\left(\left|\mathbf{y}-\mathbf{y}_{0}\right|\right) g\left(\mathbf{y}, \mathbf{y}_{0}\right) \text { belongs to } C^{\infty}\left(\mathbb{R}^{d}\right) .
$$

We claim that $v:=L_{k}(-\phi)$ satisfies

$$
\mathcal{G}_{\Omega_{q}} v=g\left(\cdot, \mathbf{y}_{0}\right) .
$$


Note that $v$ vanishes outside of $B_{\epsilon}\left(\mathbf{y}_{0}\right)$ and that $g\left(\cdot, \mathbf{y}_{0}\right)=\phi$ outside of $B_{\epsilon}\left(\mathbf{y}_{0}\right)$. Using the integration by parts formula for $L_{k}$ (Proposition 2.3) we obtain for every $\mathbf{x} \in \mathbb{R}^{d} \backslash \overline{B_{\epsilon}\left(\mathbf{y}_{0}\right)}$

$$
\begin{aligned}
\left(\mathcal{G}_{\Omega_{q}} v\right)(\mathbf{x})= & \int_{B_{\epsilon}\left(\mathbf{y}_{0}\right)} g(\mathbf{x}, \mathbf{y}) L_{k}(-\phi)(\mathbf{y}) d \mathbf{y} \\
= & \int_{B_{\epsilon}\left(\mathbf{y}_{0}\right)}\left(L_{k}^{\top} g(\mathbf{x}, \cdot)\right)(\mathbf{y})(-\phi)(\mathbf{y}) d \mathbf{y} \\
& +\left\{\oint_{\partial B_{\epsilon}\left(\mathbf{y}_{0}\right)}\left[g(\mathbf{x}, \mathbf{y}) \nabla(-\phi)(\mathbf{y})-(-\phi(\mathbf{y})) \nabla_{\mathbf{y}} g(\mathbf{x}, \mathbf{y})\right] \cdot \mathbf{n} d s(\mathbf{y})\right. \\
& +|\mathbf{m}|^{2} \oint_{\partial B_{\epsilon}\left(\mathbf{y}_{0}\right)}(-\phi)(\mathbf{y}) \frac{\partial g(\mathbf{x}, \mathbf{y})}{\partial y_{1}} n_{1}-g(\mathbf{x}, \mathbf{y}) \frac{\partial(-\phi)(\mathbf{y})}{\partial y_{1}} n_{1} d s(\mathbf{y}) \\
& \left.+2 i k|\mathbf{m}| \oint_{\partial B_{\epsilon}\left(\mathbf{y}_{0}\right)} g(\mathbf{x}, \mathbf{y})(-\phi)(\mathbf{y}) n_{1} d s(\mathbf{y})\right\}
\end{aligned}
$$

The volume integral vanishes, since $L_{k}^{\top} g(\mathbf{x}, \cdot)=0$ on $B_{\epsilon}\left(\mathbf{y}_{0}\right)$. Since $\phi$ solves the homogeneous convected Helmholtz equation outside of $B_{\epsilon}\left(\mathbf{y}_{0}\right)$, we can apply the representation formula (Theorem 2.14) for the remaining boundary integrals and obtain

$$
\{\ldots\}=\phi(\mathbf{x})=g\left(\mathbf{x}, \mathbf{y}_{0}\right) \text {. }
$$

By the spectral theorem for compact self-adjoint operators (see e.g. Alt16, 12.12 +12.13 p.395 ff.]), there exists a decreasing sequence of eigenvalues $\lambda_{j}>0$ and an orthonormal set of eigenfunctions $\psi_{j}$ of $\mathcal{C}(q)$ such that for any $v \in L^{2}(\mathbb{M})$ we have

$$
(\mathcal{C}(q))(v)=\sum_{j=1}^{\infty} \lambda_{j}\left\langle v, \psi_{j}\right\rangle_{L^{2}(\mathbb{M})} \psi_{j} .
$$

Using the spectral representation (4.21), we can also represent $(\mathcal{C}(q))^{1 / 2}$ by

$$
(\mathcal{C}(q))^{1 / 2}(v)=\sum_{j=1}^{\infty} \sqrt{\lambda_{j}}\left\langle v, \psi_{j}\right\rangle_{L^{2}(\mathbb{M})} \psi_{j} .
$$

Note that the square root $(\mathcal{C}(q))^{1 / 2}$ is also compact. Following again the principles presented in [KG07] we then get a representation of the indicator function of $\Omega_{q}$.

Corollary 4.17 (Indicator function of $\Omega_{q}$ ).

The indicator function of $\Omega_{q}$

$$
\mathbb{1}_{\Omega_{q}}\left(\mathbf{y}_{0}\right)= \begin{cases}1 & \text { if } \mathbf{y}_{0} \in \Omega_{q} \\ 0 & \text { if } \mathbf{y}_{0} \notin \Omega_{q}\end{cases}
$$


can be represented by

$$
\mathbb{1}_{\Omega_{q}}\left(\mathbf{y}_{0}\right)=\operatorname{sgn}\left(\inf \left\{\left|\langle w, \mathcal{C}(q) w\rangle_{L^{2}(\mathbb{M})}\right|: w \in L^{2}(\mathbb{M}),\left\langle w, g\left(\cdot, \mathbf{y}_{0}\right)\right\rangle_{L^{2}(\mathbb{M})}=1\right\}\right) .
$$

Moreover, we have another representation by

$$
\mathbb{1}_{\Omega_{q}}\left(\mathbf{y}_{0}\right)=\operatorname{sgn}\left(\left[\sum_{j=1}^{\infty} \frac{1}{\lambda_{j}}\left|\left\langle g\left(\cdot, \mathbf{y}_{0}\right), \psi_{j}\right\rangle_{L^{2}(\mathbb{M})}\right|^{2}\right]^{-1}\right),
$$

with the convention $\infty^{-1}:=0$.

Proof. The first statement is a direct consequence of Theorem 4.16 as $\mathbf{y}_{0} \in \Omega_{q}$ if and only if the infimum is strictly positive.

For the second statement note first that $\operatorname{ker}(\mathcal{C}(q))=\operatorname{ker}\left(\mathcal{C}^{1 / 2}(q)^{*}\right)$ and hence $\overline{\operatorname{ran}\left(\mathcal{C}^{1 / 2}(q)\right)}=\operatorname{ker}(\mathcal{C}(q))^{\perp}$. Moreover, if $\phi \in L^{2}(\mathbb{M})$ belongs to $\operatorname{ker}(\mathcal{C}(q))$ we get

$$
0=\langle\mathcal{C}(q) \phi, \phi\rangle_{L^{2}(\mathbb{M})}=\left\langle q \mathcal{G}^{*} \phi, \mathcal{G}^{*} \phi\right\rangle_{L^{2}(\Omega)} \geq q_{0} \int_{\Omega_{q}}\left|\left(\mathcal{G}^{*} \phi\right)(\mathbf{y})\right|^{2} d \mathbf{y}
$$

And hence by analytic continuation $\left(\mathcal{G}^{*} \phi\right)(\mathbf{y})=0$ for all $\mathbf{y} \in \Omega$ i.e. $\phi \in \operatorname{ker}\left(\mathcal{G}^{*}\right)$. Taking the inner product of $\phi \in \operatorname{ker}(\mathcal{C}(q))$ and $g\left(\cdot, \mathbf{y}_{0}\right)$ we get further

$$
\left\langle\phi, g\left(\cdot, \mathbf{y}_{0}\right)\right\rangle_{L^{2}(\mathbb{M})}=\int_{\mathbb{M}} \phi(\mathbf{x}) \overline{g\left(\mathbf{x}, \mathbf{y}_{0}\right)} d \mathbf{x}=\left(\mathcal{G}^{*} \phi\right)\left(\mathbf{y}_{0}\right)=0
$$

The last equation together with the previous statements shows that $g\left(\cdot, \mathbf{y}_{0}\right) \in \overline{\operatorname{ran}\left(\mathcal{C}^{1 / 2}(q)\right)}$. By Theorem 4.16 we have that $\mathbf{y}_{0} \in \Omega_{q}$ if and only if there exists a solution $\varphi \in L^{2}(\Omega)$ to the equation

$$
(\mathcal{C}(q))^{1 / 2} \varphi=g\left(\cdot, \mathbf{y}_{0}\right) .
$$

By the Picard criterion [Kir11, Theorem A.54] Equation 4.25] is solvable if and only if $g\left(\cdot, \mathbf{y}_{0}\right) \in \overline{\operatorname{ran}\left(\mathcal{C}^{1 / 2}(q)\right)}$ and

$$
\sum_{j=1}^{\infty} \frac{1}{\lambda_{j}}\left|\left\langle g\left(\cdot, \mathbf{y}_{0}\right), \psi_{j}\right\rangle_{L^{2}(\mathbb{M})}\right|^{2}<\infty
$$

which yields the claim.

Theorem 4.16 and Corollary 4.17 motivate an imaging functional of the form 4.24 or

$$
\mathcal{I}^{\mathrm{fac}}\left(\mathbf{y}_{0}\right)=\left[\sum_{j=1}^{\infty} \frac{1}{\lambda_{j}}\left|\left\langle g\left(\cdot, \mathbf{y}_{0}\right), \psi_{j}\right\rangle_{L^{2}(\mathbb{M})}\right|^{2}\right]^{-1}
$$


Moreover, in a discrete setting, the covariance matrix $\underline{C} \in \mathbb{C}^{n_{\text {mic }} \times n_{\text {mic }}}$ is often positive definite. In that case we can consider the imaging functional

$$
{\underline{\underline{\mathcal{I}^{f a c}}}}^{\mathrm{fac}}\left(\mathbf{y}_{0}\right)=\left[\sum_{j=1}^{n_{\text {mic }}} \frac{1}{\lambda_{j}}\left|\underline{g}\left(\mathbf{y}_{0}\right)^{*} \underline{\psi}_{j}\right|^{2}\right]^{-1}
$$

where $\left\{\left(\lambda_{j}, \underline{\psi}_{j}\right)\right\}_{j=1}^{n_{\text {mic }}}$ denotes the set of eigenpairs of $\underline{C}$ with normalized eigenvectors $\underline{\psi}_{j}$ and $g\left(\mathbf{y}_{0}\right)$ the propagation vector defined in (4.5). Note further that

$$
\left[\sum_{j=1}^{n_{\text {mic }}} \frac{1}{\lambda_{j}}\left|\underline{g}\left(\mathbf{y}_{0}\right)^{*} \underline{\psi}_{j}\right|^{2}\right]^{-1}=\left\|\underline{C}^{-1 / 2} \underline{g}\left(\mathbf{y}_{0}\right)\right\|_{2}^{-2}=\left(\underline{g}\left(\mathbf{y}_{0}\right)^{*} \underline{C}^{-1} \underline{g}\left(\mathbf{y}_{0}\right)\right)^{-1} .
$$

This is actually a well-known method in the field of seismic imaging, known as Capon's method Cap69. In the context of array imaging of acoustic sources this method is often referred to as adaptive Beamforming or minimum variance method. This is somewhat astounding, since in the original article by Capon, the method is derived from a completely different perspective (increasing the wavenumber resolution of the array imaging functional).

\section{Linear beamformer with minimal output}

A class of linear imaging functionals that are often considered in practice are beamformers with unit gain (c.f. Section 5.1 and Sij04]). Transferring this idea to our infinite dimensional setup this leads to imaging functionals of the type

$$
\mathcal{I}_{w}\left(\mathbf{y}_{0}\right)=\langle w, \mathcal{C}(q) w\rangle_{L^{2}(\mathbb{M})},
$$

where the function $w \in L^{2}(\mathbb{M})$ must satisfy the normalization condition

$$
\left\langle w, g\left(\cdot, \mathbf{y}_{0}\right)\right\rangle_{L^{2}(\mathbb{M})}=1 .
$$

In the array imaging context, this condition is usually called unit gain. It ensures that if the correlation data is noiseless and generated by a single monopole, the correct source power is recovered. I.e. let $\mathcal{C}(q)=\mu \mathcal{P}_{\mathbf{y}_{0}}$ with $\mu>0$, then we obtain

$$
\mathcal{I}_{w}\left(\mathbf{y}_{0}\right)=\mu\left\langle w, \mathcal{P}_{\mathbf{y}_{0}} w\right\rangle_{L^{2}(\mathbb{M})}=\mu\left(\int_{\mathbb{M}} w\left(\mathbf{x}_{1}\right) \overline{g\left(\mathbf{x}_{1}, \mathbf{y}_{0}\right)} d \mathbf{x}_{1}\right)\left(\int_{\mathbb{M}} g\left(\mathbf{x}_{2}, \mathbf{y}_{0}\right) \overline{w\left(\mathbf{x}_{2}\right)} d \mathbf{x}_{2}\right)=\mu .
$$

Often, the method of Capon is then characterized by the method that generates the minimum output of that class. In our function space setting this translates as

$$
\left.\mathcal{I}^{\text {Cap }}\left(\mathbf{y}_{0}\right)=\inf \left\{\left|\langle w, \mathcal{C}(q) w\rangle_{L^{2}(\mathbb{M})}\right|: w \in L^{2}(\mathbb{M}), w \text { satisfies } 4.29\right\}\right\} .
$$


This is exactly the infimum from the inf-criterion (Theorem 4.16, (iii)), i.e.

$$
\operatorname{sgn}\left(\mathcal{I}^{\mathrm{Cap}}\left(\mathbf{y}_{0}\right)\right)=\mathbb{1}_{\Omega_{q}}\left(\mathbf{y}_{0}\right)
$$

in our continuous setting with noise-free data. Note that Capon's method therefore only reconstructs the support of the source power function $q$ and does not recover the exact source power values.

\subsection{Discrete correlation estimation}

We have already seen discrete formulations of reconstruction methods in the last section. In this section we will present a modeling of the discrete measurement and correlation estimation process that reflects the usual procedures in practice.

\subsubsection{Maximum likelihood estimation of the covariance matrix}

For each microphone position $\mathbf{x}_{m}$ the measured data consists of a time series of relative pressure fluctuations with a total length of $n_{T} \in \mathbb{N}$ time samples. Assuming equidistant time instances, yields time data of the form

$$
p\left(\mathbf{x}_{m}, n \Delta t\right) \quad n=0, \ldots, n_{T}-1
$$

where $\Delta t$ denotes the sampling interval. Typical values for the sampling interval are of the order $\mathcal{O}\left(10^{-5} \mathrm{~s}\right)$ and the sampling frequency is defined as $f_{s}=\frac{1}{\Delta t}$. In experimental aeroacoustics, the correlation estimation in frequency domain from the discrete time data is usually carried out by Welch's method WWel67. which is outlined in the following.

1. Generate block samples: The data is divided into $n_{\text {samp }}$ time blocks with $n_{\text {time }}$ samples each where $n_{\text {time }}$ is often chosen as a power of 2 and usually $n_{\text {time }}$ is much smaller than $n_{T}$. Furthermore the block samples may be chosen overlapping in time. A common choice for the size of the overlap is half of the block sample size i.e. $\frac{n_{\text {time }}}{2}$.

2. Window block samples: Each block sample signal $\underline{p}^{(j)}\left(\mathbf{x}_{m}\right) \in \mathbb{R}^{n_{\text {time }}}$ is modified component-wise by a window function w. Examples for common window functions are

$$
\begin{aligned}
& \mathrm{w}(\cdot)_{n}=(\cdot)_{n} \text { for: } n=1, \ldots, n_{\text {time }} \quad(\text { rectangular window }), \\
& \mathrm{w}(\cdot)_{n}=\frac{1}{2}\left[1-\cos \left(\frac{2 \pi(n-1)}{n_{\text {time }}-1}\right)\right](\cdot)_{n} \text { for: } n=1, \ldots, n_{\text {time }} \quad \text { (Hann window) } .
\end{aligned}
$$

3. Discrete Fourier transform: Apply the discrete Fourier transform (DFT) on the windowed time signals, which yields block samples in frequency domain

$$
\hat{p}^{(j)}\left(\mathbf{x}_{m}, \omega_{k}\right)=\left[\operatorname{DFT}\left(\mathrm{w}\left(\underline{p}^{(j)}\left(\mathbf{x}_{m}\right)\right)\right)\right]_{k} \quad \text { for: } k=-\frac{n_{\text {time }}}{2}, \ldots, \frac{n_{\text {time }}}{2}-1 .
$$


The discrete angular frequencies are defined by $\omega_{k}=\frac{2 \pi k}{\Delta t n_{\mathrm{time}}}$ and

$$
\left[\operatorname{DFT}\left(\mathrm{w}\left(\underline{p}^{(j)}\left(\mathbf{x}_{m}\right)\right)\right)\right]_{k}=\sum_{n=1}^{n_{\text {time }}} e^{-i n \Delta t \omega_{k}}\left[\mathrm{w}\left(\underline{p}^{(j)}\left(\mathbf{x}_{m}\right)\right)\right]_{n} .
$$

4. Sample correlation: For a fixed frequency $\omega_{k}$ define the microphone vectors $\underline{\hat{p}}^{(j)}\left(\omega_{k}\right)=\left(\hat{p}^{(j)}\left(\mathbf{x}_{1}, \omega_{k}\right), \ldots, \hat{p}^{(j)}\left(\mathbf{x}_{n_{\text {mic }}}, \omega_{k}\right)\right)^{\top}$. The empirical estimate of the correlation matrix is then given by

$$
\underline{C}^{\mathrm{obs}}=\frac{1}{n_{\mathrm{samp}}} \sum_{j=1}^{n_{\mathrm{samp}}} \underline{\hat{p}}^{(j)}\left(\omega_{k}\right) \underline{\hat{p}}^{(j) *}\left(\omega_{k}\right) .
$$

For the mathematical analysis we make the following assumptions regarding the pressure signals in frequency domain.

Assumption 4.18 (Pressure signals).

The pressure vectors $\left\{\underline{\hat{p}}^{(j)}\right\}_{j=1}^{n_{\text {samp }}}$ are drawn independently from a complex valued Gaussian random variable $\underline{\hat{p}}$ with zero mean and covariance matrix $\underline{C}$ i.e.

$$
\underline{\hat{p}} \sim \mathcal{N}_{\mathbb{C}}(0, \underline{C}) .
$$

For a concise introduction to the theory of complex random variables and the complex normal distribution we refer to AHSE95. To distinguish between real and complex Gaussian distributions, they will be denoted by $\mathcal{N}_{\mathbb{R}}$ and $\mathcal{N}_{\mathbb{C}}$ respectively.

Under the above assumptions, the likelihood function for the data $\left\{\underline{\hat{p}}^{(j)}\right\}_{j=1}^{n_{\text {samp }}}$ is given by

$$
\mathcal{L}(\underline{C})=\pi^{-n_{\text {mic }} n_{\text {samp }}} \operatorname{det}(\underline{C})^{-n_{\text {samp }}} \exp \left(-\sum_{j=1}^{n_{\text {samp }}} \underline{\hat{p}}^{(j) *} \underline{C}^{-1} \underline{\hat{p}}^{(j)}\right) .
$$

The maximum likelihood estimator of the covariance matrix $\underline{C}$ is defined as the solution to the following optimization problem

$$
\begin{aligned}
\text { maximize } & \mathcal{L}(\underline{C}) \\
\text { subject to } & \underline{C} \text { is Hermitian and positive semi-definite. }
\end{aligned}
$$

The maximizer of problem 4.31 is given by the sample covariance matrix $\underline{C}^{\text {obs }}$ (see e.g. [AHSE95, Theorem 4.1]). 


\subsubsection{Discrete noise model}

We may decompose the correlation data into the sum of the true data and a noise term

$$
\underline{C}^{\mathrm{obs}}=\underline{C}+\left(\underline{C}^{\mathrm{obs}}-\underline{C}\right)=\underline{C}+Z .
$$

where $\underline{C}$ is deterministic and the noise matrix $Z$ a matrix-valued random variable with $\mathbb{E}(Z)=0$. Since we do not want to deal with matrix-valued random variables, we consider their vectorized versions instead. Therefore, for any $A \in \mathbb{C}^{d \times d}$ the column-wise vectorization is defined as

$$
\operatorname{vec}(A)=\left(A_{11}, \ldots, A_{d 1}, A_{12}, \ldots, A_{d 2}, \ldots, A_{1 d}, \ldots, A_{d d}\right)^{\top} .
$$

Thus, vec $(Z) \in \mathbb{C}^{n_{\text {mic }}^{2}}$ is a complex vector-valued random variable with zero mean. The positive semi-definite covariance matrix of $\operatorname{vec}(Z)$ is given by means of the covariance matrix of correlations

$$
\Sigma:=\operatorname{Cov}\left(\operatorname{vec}\left(\hat{p} \hat{p}^{*}\right)\right)
$$

as

$$
\operatorname{Cov}(\operatorname{vec}(Z))=\mathbb{E}\left(\operatorname{vec}(Z) \operatorname{vec}(Z)^{*}\right)=\frac{1}{n_{\text {samp }}} \Sigma
$$

Since $\underline{C}$ is deterministic, Equation 4.32 implies

$$
\operatorname{Cov}\left(\operatorname{vec}\left(\underline{C}^{\text {obs }}\right)\right)=\operatorname{Cov}(\operatorname{vec}(Z))=\frac{1}{n_{\text {samp }}} \Sigma
$$

The entries of $\Sigma$ are of the form

$$
\begin{aligned}
& n_{\text {samp }} \operatorname{Cov}\left(\frac{1}{n_{\text {samp }}} \sum_{j=1}^{n_{\text {samp }}} \hat{p}_{m}^{(j)} \hat{p}_{l}^{(j) *}, \frac{1}{n_{\text {samp }}} \sum_{j=1}^{n_{\text {samp }}} \hat{p}_{m^{\prime}}^{(j)} \hat{p}_{l^{\prime}}^{(j) *}\right) \\
= & \operatorname{Cov}\left(\hat{p}_{m} \hat{p}_{l}^{*}, \hat{p}_{m^{\prime}} \hat{p}_{l^{\prime}}^{*}\right) \\
= & \mathbb{E}\left(\hat{p}_{m} \hat{p}_{l}^{*} \hat{p}_{m^{\prime}}^{*} \hat{p}_{l^{\prime}}\right)-\mathbb{E}\left(\hat{p}_{m} \hat{p}_{l}^{*}\right) \mathbb{E}\left(\hat{p}_{m^{\prime}} \hat{p}_{l^{\prime}}^{*}\right)^{*} \quad \text { for } m, m^{\prime}, l, l^{\prime}=1, \ldots n_{\text {mic }} .
\end{aligned}
$$

Hence, constructing the data covariance matrix $\Sigma$, requires the knowledge of fourthorder moments of the pressure vector $\hat{p}$. Under Assumption 4.18, Isserlis' theorem (for the case of fourth-order moments) [Iss16] is applicable and provides an explicit formula. For our scenario that yields

$$
\mathbb{E}\left(\hat{p}_{m} \hat{p}_{l}^{*} \hat{p}_{m^{\prime}}^{*} \hat{p}_{l^{\prime}}\right)=\mathbb{E}\left(\hat{p}_{m} \hat{p}_{l}^{*}\right) \mathbb{E}\left(\hat{p}_{m^{\prime}}^{*} \hat{p}_{l^{\prime}}\right)+\mathbb{E}\left(\hat{p}_{m} \hat{p}_{m^{\prime}}^{*}\right) \mathbb{E}\left(\hat{p}_{l}^{*} \hat{p}_{l^{\prime}}\right)+\mathbb{E}\left(\hat{p}_{m} \hat{p}_{l^{\prime}}\right) \mathbb{E}\left(\hat{p}_{l}^{*} \hat{p}_{m^{\prime}}^{*}\right)
$$

Combining the last two equations yields

$$
\operatorname{Cov}\left(\hat{p}_{m} \hat{p}_{l}^{*}, \hat{p}_{m^{\prime}} \hat{p}_{l^{\prime}}^{*}\right)=\mathbb{E}\left(\hat{p}_{m} \hat{p}_{m^{\prime}}^{*}\right) \mathbb{E}\left(\hat{p}_{l}^{*} \hat{p}_{l^{\prime}}\right)+\mathbb{E}\left(\hat{p}_{m} \hat{p}_{l^{\prime}}\right) \mathbb{E}\left(\hat{p}_{l} \hat{p}_{m^{\prime}}\right)^{*} .
$$


For a deeper discussion of Formula (4.34) and its versions for further higher-order moments we refer to GB04] and [FGHB14]. Formula (4.34) reveals that the data covariance matrix $\Sigma$ may be estimated by using only second-order moments of the data. Inserting the corresponding empirical estimates yields

$$
\operatorname{Cov}\left(\hat{p}_{m} \hat{p}_{l}^{*}, \hat{p}_{m^{\prime}} \hat{p}_{l^{\prime}}^{*}\right) \approx \underline{C}_{m m^{\prime}}^{\mathrm{obs}} \underline{C}_{l l^{\prime}}^{\mathrm{obs}}{ }^{*}+\underline{C}_{m l^{\prime}}^{\mathrm{ps}} \underline{C}_{m^{\prime} l}^{\mathrm{ps}},
$$

with the empirical pseudo correlation matrix

$$
\underline{C}^{\mathrm{ps}}=\frac{1}{n_{\mathrm{samp}}} \sum_{j=1}^{n_{\mathrm{samp}}} \underline{\hat{p}}^{(j)} \underline{\hat{p}}^{(j)^{\top}} .
$$

Another straightforward possibility to estimate $\Sigma$ is given by the data sample covariance matrix (multiplied by the number of block samples $n_{\text {samp }}$ )

$$
\Sigma \approx \sum_{j=1}^{n_{\mathrm{samp}}}\left[\operatorname{vec}\left(\underline{C}^{\mathrm{obs}(j)}\right)-\operatorname{vec}\left(\underline{C}^{\mathrm{obs}}\right)\right]\left[\operatorname{vec}\left(\underline{C}^{\mathrm{obs}(j)}\right)-\operatorname{vec}\left(\underline{C}^{\mathrm{obs}}\right)\right]^{*},
$$

which does not rely on any statistical assumptions on the pressure samples. For further statistical evaluations, additional properties of the estimated data covariance matrix $\Sigma^{\text {est }}$ are desirable.

Hermitian: Both approximation formulas 4.35 and (4.36) ensure that $\Sigma^{\text {est }}$ is Hermitian.

Positive semi-definite (psd): If $\Sigma^{\text {est }}$ is estimated by Formula (4.36), the result is always psd as it is the sum of psd matrices. Formula (4.35) does not ensure a positive semidefinite result.

Regular: Formula 4.35 does not ensure regularity. Formula 4.36 reveals that the rank of the resulting matrix is at most $n_{\text {samp }}$ (due to the rank inequality) and hence whenever $n_{\text {samp }}<n_{\text {mic }}^{2}$ the matrix cannot be regular.

For practical applications an uncertainty quantification of the correlation estimations provides valuable information on the quality of the data. One interesting measure may be the stochastic noise level of the correlation data which is defined by the root mean square deviation

$$
\delta^{\mathrm{rms}}\left(\underline{C}^{\mathrm{obs}}\right):=\sqrt{\mathbb{E}\left(\sum_{m, l=1}^{n_{\mathrm{mic}}}\left|\underline{C}_{m l}^{\mathrm{obs}}-\underline{C}_{m l}\right|^{2}\right)}
$$


Using the definitions of $\Sigma$ and the noise term $Z$ we obtain further

$$
\delta^{\mathrm{rms}}\left(\underline{C}^{\mathrm{obs}}\right)=\sqrt{\mathbb{E}\left(\sum_{m, l=1}^{n_{\mathrm{mic}}}\left|Z_{m l}\right|^{2}\right)}=\sqrt{\sum_{m, l=1}^{n_{\mathrm{mic}}} \operatorname{Var}\left(Z_{m l}\right)}=\sqrt{\frac{1}{n_{\mathrm{samp}}} \operatorname{tr}(\Sigma)} .
$$





\section{Chapter}

\section{Weighted Data Spaces}

The results that are presented in this chapter are mostly published in the article [RSHE20].

Based on the discrete formulations of the last chapter we will introduce the concept of weighted data norms as presented in [RSHE20]. This framework is applicable to all imaging methods presented so far (i.e. Beamforming, DAMAS and CMF). Several explicit weighting choices are discussed and analyzed.

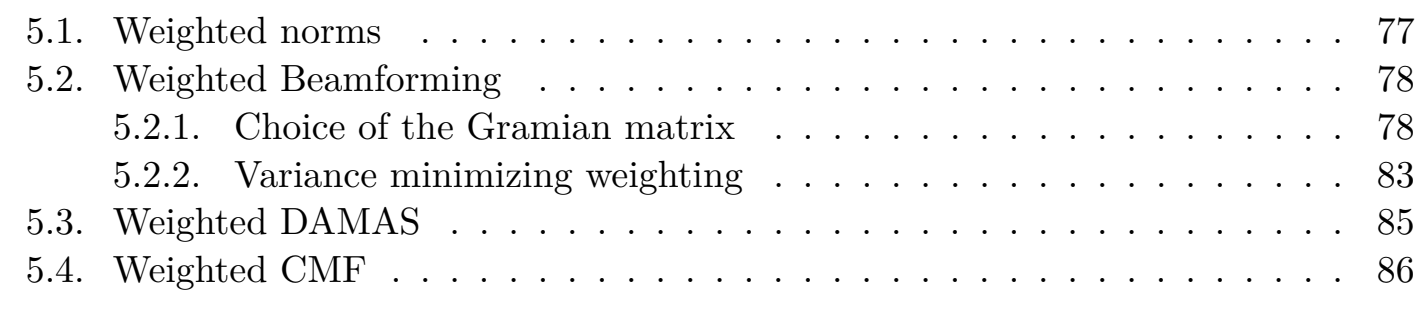

\subsection{Weighted norms}

The discrete versions of source reconstruction methods introduced in Section 4.1 (see (4.3) and (4.6) $)$ are formulated on the finite-dimensional Hilbert space $\left(\mathbb{C}^{n_{\text {mic }} \times n_{\text {mic }}},\langle\cdot, \cdot\rangle_{F}\right) \simeq$ $\left(\mathbb{C}^{n_{\text {mic }}^{2}},\langle\cdot, \cdot\rangle_{2}\right)$ with inner product

$$
\langle A, B\rangle_{F}=\sum_{m, l=1}^{n_{\text {mic }}} A_{m l}\left(B_{m l}\right)^{*}=\sum_{j=1}^{n_{\text {mic }}^{2}} \operatorname{vec}(A)_{j}\left(\operatorname{vec}(B)_{j}\right)^{*}=\langle\operatorname{vec}(A), \operatorname{vec}(B)\rangle_{2} .
$$

Any inner product on $\mathbb{C}^{n_{\text {mic }}^{2}}$ can be characterized by its Gramian matrix (see e.g. [RAH12] which is Hermitian, positive definite and will be denoted by $W$ in the follow- 
ing. For each Hermitian, positive definite matrix $W$ we define the space $\left(\mathbb{C}^{n_{\text {mic }}^{2}},\langle\cdot, \cdot\rangle_{W}\right)$ via its inner product

$$
\langle X, Y\rangle_{W}=\left\langle W^{-1 / 2} X, W^{-1 / 2} Y\right\rangle_{2}=\left\langle X, W^{-1} Y\right\rangle_{2} \quad \text { for } X, Y \in \mathbb{C}^{M^{2}}
$$

With the eigenvalue decomposition (EVD) of $W$ given by

$$
W=U \operatorname{diag}\left(\lambda_{1}, \ldots, \lambda_{M^{2}}\right) U^{*},
$$

the matrix $W^{-1 / 2}$ is defined by

$$
W^{-1 / 2}=U \operatorname{diag}\left(\lambda_{1}^{-1 / 2}, \ldots, \lambda_{n_{\text {mic }}^{2}}^{-1 / 2}\right) U^{*} .
$$

The corresponding norm is defined as

$$
\|X\|_{W}=\sqrt{\langle X, X\rangle_{W}} \text { for } X \in \mathbb{C}^{n_{\text {mic }}^{2}} .
$$

\subsection{Weighted Beamforming}

Recall that Conventional Beamforming $(\mathrm{CBF})$ is characterized by a minimization problem on $\left(\mathbb{C}^{n_{\text {mic }} \times n_{\text {mic }}},\langle\cdot, \cdot\rangle_{F}\right)$ (see 4.6$)$ ). If we consider a generic Hilbert space with Gramian matrix $W$ instead, a generalized Beamforming functional is defined by

$$
\underline{\underline{I}}_{W}(\mathbf{y})=\underset{\mu \in \mathbb{C}}{\operatorname{argmin}}\left\|\operatorname{vec}\left(\underline{C}^{\text {obs }}\right)-\mu \operatorname{vec}(\underline{\mathcal{P}})\right\|_{W}^{2} .
$$

In analogy to $\mathrm{CBF}$ the solution to $(5.2)$ is given by

$$
\underline{\mathcal{I}}_{W}(\mathbf{y})=\frac{\left\langle\operatorname{vec}\left(\underline{C}^{\text {obs }}\right), \operatorname{vec}\left(\underline{\mathcal{P}_{\mathbf{y}}}\right)\right\rangle_{W}}{\left\|\operatorname{vec}\left(\underline{\mathcal{P}_{\mathbf{y}}}\right)\right\|_{W}^{2}} .
$$

\subsubsection{Choice of the Gramian matrix}

The generic beamformer in $(5.2)$ is defined for any Hermitian, positive definite Gramian matrix $W$. In the following we will mostly use the term weighting matrix instead of Gramian matrix. We will discuss various particular choices for $W$ which incorporates several common imaging methods into the generic framework presented above. Furthermore, weighting choices that depend on the data covariances (introduced in Section 4.3.2 will be considered. 


\section{Conventional Beamforming:}

$$
W=\sigma^{2} I \text { for } \sigma^{2}>0
$$

If $W$ is a positive multiple of the identity matrix, the induced norm $\|\cdot\|_{W}$ is just the standard Euclidean norm times the factor $\sigma^{2}$. Hence, the minimization problem 5.2 is equivalent to

$$
\underline{\mathcal{I}}_{W}(\mathbf{y})=\underset{\mu \in \mathbb{C}}{\operatorname{argmin}}\left\|\operatorname{vec}\left(C^{\mathrm{obs}}\right)-\mu \operatorname{vec}\left(\underline{\mathcal{P}_{\mathbf{y}}}\right)\right\|_{2}^{2},
$$

which is exactly the CBF solution. For the remainder of this section, we will omit the dependency on the focus point $\mathbf{y}$ to increase the readability.

Diagonal inverse covariance weighting (iv-d):

$$
W_{i j}= \begin{cases}\Sigma_{i j} & \text { if } i=j \\ 0 & \text { else }\end{cases}
$$

Recall that $\Sigma$ denotes the covariance matrix of pressure correlations (see Equation (4.33)). The weighting (5.5) is based on the variances of the CSM components

$$
\sigma_{m l}^{2}=\operatorname{Var}\left(\underline{C}_{m l}^{\mathrm{obs}}\right) .
$$

As $W$ is a diagonal matrix, the induced norm may be rewritten in matrix notation

$$
\|\operatorname{vec}(A)\|_{W}^{2}=\sum_{m=1}^{n_{\text {mic }}} \sum_{l=1}^{n_{\text {mic }}} \frac{1}{\sigma_{m l}^{2}}\left|A_{m l}\right|^{2} \quad \text { for } A \in \mathbb{C}^{n_{\text {mic }} \times n_{\text {mic }}} .
$$

This is a modified Frobenius norm, where each component is weighted by its inverse variance. This may be interpreted as a reliability weighting on the empirical correlation data. Components that exhibit a high variance are considered as less reliable than components with a low variance. Thus, components with low variances have a larger impact on the imaging result than components with high variances.

Full inverse covariance weighting (iv-f):

In that case the weighting matrix $W$ is the entire data covariance matrix i.e.

$$
W=\Sigma .
$$

The distance measure

$$
\mathrm{d}(X, Y):=\|X-Y\|_{\Sigma} \quad \text { for } X, Y \in \mathbb{C}^{n_{\text {mic }}^{2}}
$$


is known as Mahalanobis distance [Mah36]. In the field of statistics, the approach of weighting a least squares problem with the data covariance matrix is known as generalized least squares [Ait36].

Remark 5.1 (Whitening).

The iv-f (5.7) weighting applies a whitening transformation on the data since we have by definition

$$
\operatorname{Cov}\left(\Sigma^{-1 / 2} \operatorname{vec}\left(\underline{C}^{\text {obs }}\right)\right)=I
$$

Moreover, the iv-d and conventional weighting perform also whitening transformations under the assumption that the vectorized noise vec $(Z)$ is uncorrelated (iv-d) respectively white (conventional).

\section{Beamforming with shading:}

From the practical point of view it is sometimes desirable to endow each microphone with an individual positive weight $\nu_{1}, \ldots, \nu_{n_{\text {mic }}}$. That may be useful if for instance different microphone types are used within the same array. Such an approach is usually referred to as shading in the literature (see e.g. [Sij10, BMP87]). A Beamforming functional with shading is defined by

$$
{\underline{\mathcal{I}^{\text {shad }}}}^{\operatorname{argmin}} \sum_{\mu \in \mathbb{C}}^{n_{\text {mic }}} \sum_{m=1}^{n_{\text {mic }}}\left|C_{m l}^{\text {obs }}-\mu \nu_{m} \nu_{l} \mathbf{g}_{m} \mathbf{g}_{l}^{*}\right|^{2} .
$$

This can be incorporated into the presented framework by the weighting matrix

$$
W=\operatorname{diag}\left(\operatorname{vec}\left(\boldsymbol{\nu} \boldsymbol{\nu}^{\top}\right)\right)^{-1},
$$

with $\boldsymbol{\nu}=\left(\nu_{1}, \ldots, \nu_{n_{\text {mic }}}\right)^{\top}$.

\section{Capon's method:}

This method was introduced by Capon Cap69 and we already discussed its relation to the factorization method in Section 4.2, It is also known as Minimum Variance Method or Adaptive Beamforming and defined by

$$
\underline{\mathcal{I}}^{\text {Cap }}=\underline{w}_{\text {Cap }}^{*} \underline{C}^{\text {obs }} \underline{w}_{\text {Cap }} .
$$

The vector $\underline{w}_{\text {Cap }}$ is implicitly defined by the solution of

$$
\min _{\underline{w} \in \mathbb{C}^{M}} \underline{w}^{*} C^{\text {obs }} \underline{w} \quad \text { subject to } \quad \underline{g}^{*} \underline{w}=1 .
$$

Note that with the random pressure vector $\underline{\hat{p}}$ we have

$$
\mathbb{E}\left[\left|\underline{w}^{*} \underline{\hat{p}}\right|^{2}\right]=\underline{w}^{*} \mathbb{E}\left[\underline{\hat{p} \hat{p}^{*}}\right] \underline{w}=\underline{w}^{*} \underline{C w} \approx \underline{w}^{*} \underline{C}^{\mathrm{obs}} \underline{w} .
$$


Since $\underline{\hat{p}}$ has zero mean by Assumption 4.18 we have

$$
\mathbb{E}\left[\left|\underline{w^{*}} \underline{\hat{p}}\right|^{2}\right]=\operatorname{Var}\left(\underline{w}^{*} \underline{\hat{p}}\right)
$$

That explains the term Minimum Variance Method (see also [JD93]). We will assume in the following that $\underline{C}^{\text {obs }}$ is regular. In that case the explicit solution of 5.9 is

$$
\underline{w}_{\mathrm{Cap}}=\frac{\left(\underline{C}^{\mathrm{obs}}\right)^{-1} \underline{g}}{\underline{g}^{*}\left(\underline{C}^{\mathrm{obs}}\right)^{-1} \underline{g}} .
$$

The fact that the vector $\underline{w}_{\text {Cap }}$ depends on the empirical correlation data explains the term Adaptive Beamforming. Inserting (5.11) into (5.8) yields (cf. Equation (4.26) and (4.27)

$$
\underline{\mathcal{I}}^{\mathrm{Cap}}=\left(\underline{g}^{*}\left(\underline{C}^{\mathrm{obs}}\right)^{-1} \underline{g}\right)^{-1}
$$

The Capon beamformer (5.12) can also be characterized by a weighting matrix. Since the derivation is not as straightforward as for the previous cases, we will formulate it as a lemma.

Lemma 5.2 (Weighting matrix for Capon's method).

If $\underline{C}^{\text {obs }}$ is regular, the Capon beamformer is characterized by

$$
W=\underline{C}^{\text {obs }}{ }^{\top} \otimes \underline{C}^{\text {obs }}
$$

where $\otimes$ denotes the Kronecker product.

Proof. For a definition of the Kronecker product see e.g. [HJ91, Def. 4.2.1 p.243]. Firstly, $W$ defined as in (5.13) is a valid choice since it is regular, Hermitian and positive definite. All those properties are inherited by $\underline{C}^{\text {obs }}$ (see [HJ91, p. $243 \mathrm{ff}$.]). By elementary properties of the Kronecker product [HJ91, Lemma 4.3 .1 p.255] we have for any $A \in$ $\mathbb{C}^{n_{\text {mic }} \times n_{\text {mic }}}$

$$
W^{-1} \operatorname{vec}(A)=\left(\underline{C}^{\mathrm{obs}}{ }^{\top} \otimes \underline{C}^{\mathrm{obs}}\right)^{-1} \operatorname{vec}(A)=\operatorname{vec}\left(\underline{C}^{\mathrm{obs}^{-1}} A \underline{C}^{\mathrm{obs}^{-1}}\right) .
$$

Using this identity for $A=\underline{C}^{\text {obs }}$ and $A=\underline{\mathcal{P}}$ yields

$$
\begin{aligned}
& \underline{\mathcal{I}}_{W}=\frac{\left\langle\operatorname{vec}\left(\underline{C}^{\mathrm{obs}}\right), \operatorname{vec}(\underline{\mathcal{P}})\right\rangle_{W}}{\|\operatorname{vec}(\underline{\mathcal{P}})\|_{W}^{2}}=\frac{\left\langle W^{-1} \operatorname{vec}\left(\underline{C}^{\mathrm{obs}}\right), \operatorname{vec}(\underline{\mathcal{P}})\right\rangle_{2}}{\left\langle\operatorname{vec}(\underline{\mathcal{P}}), W^{-1} \operatorname{vec}(\underline{\mathcal{P}})\right\rangle_{2}} \\
& =\frac{\left\langle\operatorname{vec}\left(\underline{C}^{\mathrm{obs}^{-1}}\right), \operatorname{vec}(\underline{\mathcal{P}})\right\rangle_{2}}{\left\langle\operatorname{vec}(\underline{\mathcal{P}}), \operatorname{vec}\left(\underline{C}^{\mathrm{obs}}{ }^{-1} \underline{\mathcal{P} C}{ }^{\mathrm{obs}}{ }^{-1}\right)\right\rangle_{2}}=\frac{\underline{g}^{*} \underline{C}^{\mathrm{obs}^{-1}} \underline{g}}{\underline{g}^{*} \underline{C}^{\mathrm{obs}^{-1}} \underline{g g}^{*} \underline{C}^{\mathrm{obs}^{-1}} \underline{g}}=\underline{\mathcal{I}}^{\text {Cap }} .
\end{aligned}
$$


If the pressure signals have additional statistical properties, the Capon and iv-f beamformer are equivalent in the following sense.

Lemma 5.3 (Relation of Capon and iv-f beamformer with exact data). In addition to Assumption 4.18 , let the pressure signal $\underline{\hat{p}}$ satisfy

$$
\mathbb{E}\left(\underline{\hat{p} \hat{p}^{\top}}\right)=0 .
$$

Then, for exact correlation data $\underline{C}$ holds

$$
\left(\underline{g}^{*} \underline{C}^{-1} \underline{g}\right)^{-1}=\frac{\langle\operatorname{vec}(\underline{C}), \operatorname{vec}(\underline{\mathcal{P}})\rangle_{\Sigma}}{\|\operatorname{vec}(\underline{\mathcal{P}})\|_{\Sigma}^{2}},
$$

i.e. the Capon beamformer (left) coincides with the iv-f beamformer (right).

Proof. From Property (5.14) and Equation (4.34) we obtain

$$
\operatorname{Cov}\left(\hat{p}_{m} \hat{p}_{l}^{*}, \hat{p}_{m^{\prime}} \hat{p}_{l^{\prime}}^{*}\right)=\mathbb{E}\left(\hat{p}_{m} \hat{p}_{m^{\prime}}^{*}\right) \mathbb{E}\left(\hat{p}_{l}^{*} \hat{p}_{l^{\prime}}\right)
$$

For the data covariance matrix $\Sigma$ that yields

$$
\Sigma=\operatorname{Cov}\left(\operatorname{vec}\left(\underline{\hat{p} \hat{p}^{*}}\right)\right)=\underline{C}^{\top} \otimes \underline{C} .
$$

In analogy to Lemma 5.2 we conclude that the expression on the right hand side is the weighting matrix that characterizes Capon's method with exact data, given by $\left(\underline{g}^{*} \underline{C}^{-1} \underline{g}\right)^{-1}$.

Remark 5.4 (Relation of Capon and iv-f beamformer with noisy data).

In a real experimental setup only the noisy CSM $\underline{C}^{\text {obs }}$ and an approximate data covariance matrix $\Sigma^{\mathrm{est}}$ are available. If the pressure signal satisfies Assumption 4.18 along with (5.14), the components of $\Sigma^{\mathrm{est}}$ may be estimated by

$$
\operatorname{Cov}\left(\hat{p}_{m} \hat{p}_{l}^{*}, \hat{p}_{m^{\prime}} \hat{p}_{l^{\prime}}^{*}\right) \approx \underline{C}_{m m^{\prime}}^{\mathrm{obs}} \underline{C l l}_{l l^{\prime}}^{\mathrm{obs}}
$$

In that case we obtain similar to Lemma 5.3

$$
\left(\underline{g}^{*} \underline{C}^{\mathrm{obs}^{-1}} \underline{g}\right)^{-1}=\frac{\left\langle\operatorname{vec}\left(\underline{C}^{\mathrm{obs}}\right), \operatorname{vec}(\underline{\mathcal{P}})\right\rangle_{\Sigma^{\mathrm{est}}}}{\|\operatorname{vec}(\underline{\mathcal{P}})\|_{\Sigma^{\mathrm{est}}}^{2}} .
$$

Complex Gaussian random variables z that satisfy

$$
\mathbb{E}(z)=0 \quad \text { and } \quad \mathbb{E}\left(z z^{\top}\right)=0
$$

are usually called proper [PJS14, Def. 2.1, p.35].

Robust Adaptive Beamforming (RAB):

For most real experimental datasets, $\underline{C}^{\text {obs }}$ is indeed regular but also ill-conditioned which 
is a drawback of Capon's method. Therefore Cox et al. [CZO87] suggested a regularized, robust version of the Capon beamformer. RAB depends on a control parameter $\mu>0$ that is added on the diagonal of $\underline{C}^{\text {obs }}$. The method is defined by

$$
\begin{aligned}
\underline{\mathcal{I}}^{\mathrm{RAB}} & =\frac{\underline{g}^{*}\left(\underline{C}^{\mathrm{obs}}+\mu I\right)^{-1} \underline{C}^{\mathrm{obs}}\left(C^{\mathrm{obs}}+\mu I\right)^{-1} \underline{g}}{\left(\underline{g}^{*}\left(\underline{C}^{\mathrm{obs}}+\mu I\right)^{-1} \underline{g}\right)^{2}} \\
& =\frac{\left\langle\operatorname{vec}\left(\underline{C}_{\mu}^{\mathrm{obs}}{ }^{-1} \underline{C}^{\mathrm{obs}} \underline{C}_{\mu}^{\mathrm{obs}}-1\right), \operatorname{vec}(\underline{\mathcal{P}})\right\rangle_{2}}{\left\langle\operatorname{vec}\left(\underline{C}_{\mu}^{\mathrm{obs}}{ }^{-1} \underline{\mathcal{P}}_{\mu}^{\mathrm{obs}}{ }^{-1}\right), \operatorname{vec}(\underline{\mathcal{P}})\right\rangle_{2}}
\end{aligned}
$$

with $\underline{C}_{\mu}^{\text {obs }}=\underline{C}^{\text {obs }}+\mu I$. RAB is characterized by the following weighting matrix

$$
W=\underline{C}_{\mu}^{\mathrm{obs}}{ }^{\top} \otimes \underline{C}_{\mu}^{\mathrm{obs}} .
$$

The derivation of 5.16 is analogous to the proof of Lemma 5.2 and will be omitted here.

\subsubsection{Variance minimizing weighting}

Recall the class of generic beamformers $\underline{I}_{W}$ defined in Equation 5.3

$$
\underline{\mathcal{I}}_{W}=\frac{\left\langle\operatorname{vec}\left(\underline{C}^{\mathrm{obs}}\right), \operatorname{vec}(\underline{\mathcal{P}})\right\rangle_{W}}{\|\operatorname{vec}(\underline{\mathcal{P}})\|_{W}^{2}} .
$$

By construction, those estimators are all linear with respect to the data. However, the class of all linear estimators is larger. For each source position $\mathbf{y}$, any $\ell \in \mathbb{C}^{n_{\text {mic }}^{2}}$ defines a linear source power estimator by.

$$
\ell^{*} \operatorname{vec}\left(\underline{C}^{\text {obs }}\right)
$$

If the exact data is given by a monopole with source power $\mu^{2}$ and hence

$$
\underline{C}^{\text {obs }}=\mu^{2} \underline{\mathcal{P}}+Z \quad \text { with } \mathbb{E}(Z)=0
$$

it is desirable, that the estimator is unbiased i.e. that

$$
\mathbb{E}\left(\ell^{*} \operatorname{vec}\left(\underline{C}^{\text {obs }}\right)\right)=\mu^{2} .
$$

For monopole data this is equivalent to

$$
\ell^{*} \underline{\mathcal{P}}=1 .
$$


Property (5.17) is often referred to as unit gain, thus we define the set of linear estimators with unit gain by $\mathcal{U}:=\left\{\ell \in \mathbb{C}^{n_{\text {mic }}^{2}}: \ell^{*} \operatorname{vec}(\underline{\mathcal{P}})=1\right\}$. Note that all beamformers that were presented in this section belong to this class by

$$
\ell_{W}=\frac{1}{\|\operatorname{vec}(\underline{\mathcal{P}})\|_{W}^{2}} W^{-1} \operatorname{vec}(\underline{\mathcal{P}}) .
$$

Since the CSM estimator $\underline{C}^{\text {obs }}$ is random, each estimator $\ell^{*} \operatorname{vec}\left(\underline{C}^{\text {obs }}\right)$ is random as well and its expected value is given by

$$
\mathbb{E}\left(\ell^{*} \operatorname{vec}\left(\underline{C}^{\text {obs }}\right)\right)=\ell^{*} \operatorname{vec}(\underline{C}),
$$

which is the estimator in case of exact data. A good estimator should therefore have only a small deviation from this ideal value i.e. it should hava a small variance

$$
\operatorname{Var}\left[\ell^{*} \operatorname{vec}\left(\underline{C}^{\text {obs }}\right)\right]=\mathbb{E}\left[\left|\ell^{*} \operatorname{vec}\left(\underline{C}^{\text {obs }}\right)-\mathbb{E}\left(\ell^{*} \operatorname{vec}\left(\underline{C}^{\text {obs }}\right)\right)\right|^{2}\right] .
$$

The next result is closely related to the Gauss-Markov Theorem for linear unbiased estimators (see e.g. [Kar04, Theorem 2.1]) and states that the iv-f beamformer $\left(\ell_{\Sigma}\right)$ has the lowest variance among all linear estimators with unit gain.

Theorem 5.5 (Strong variance optimality of iv-f among $\mathcal{U}$ ).

Assume that the data covariance matrix $\Sigma=\operatorname{Cov}\left(\operatorname{vec}\left(\underline{C}^{\text {obs }}\right)\right)$ is regular, then for any $\ell \in \mathcal{U}$ with $\ell \neq \ell_{\Sigma}$ we have

$$
\operatorname{Var}\left(\ell_{\Sigma}^{*} \operatorname{vec}\left(\underline{C}^{\text {obs }}\right)\right)<\operatorname{Var}\left(\ell^{*} \operatorname{vec}\left(\underline{C}^{\text {obs }}\right)\right) .
$$

Proof. For arbitrary $\ell \in \mathcal{U}$ with $\ell \neq \ell_{\Sigma}$ we obtain the following identity

$$
\begin{aligned}
\operatorname{Var}\left(\ell^{*} \operatorname{vec}\left(\underline{C}^{\mathrm{obs}}\right)\right)= & \operatorname{Var}\left(\ell_{\Sigma}^{*} \operatorname{vec}\left(\underline{C}^{\mathrm{obs}}\right)\right)+\operatorname{Var}\left(\left(\ell^{*}-\ell_{\Sigma}^{*}\right) \operatorname{vec}\left(\underline{C}^{\mathrm{obs}}\right)\right) \\
& +2 \operatorname{Re}\left(\operatorname{Cov}\left(\left(\ell^{*}-\ell_{\Sigma}^{*}\right) \operatorname{vec}\left(\underline{C}^{\mathrm{obs}}\right), \ell_{\Sigma}^{*} \operatorname{vec}\left(\underline{C}^{\mathrm{obs}}\right)\right)\right) .
\end{aligned}
$$

The last summand vanishes since

$$
\begin{aligned}
& \operatorname{Cov}\left(\left(\ell^{*}-\ell_{\Sigma}^{*}\right) \operatorname{vec}\left(\underline{C}^{\mathrm{obs}}\right), \ell_{\Sigma}^{*} \operatorname{vec}\left(\underline{C}^{\mathrm{obs}}\right)\right) \\
= & \left(\ell^{*}-\ell_{\Sigma}^{*}\right) \mathbb{E}\left(\operatorname{vec}\left(\underline{C}^{\mathrm{obs}}\right) \operatorname{vec}\left(\underline{C}^{\mathrm{obs}}\right)^{*}\right) \ell_{\Sigma}-\left(\ell^{*}-\ell_{\Sigma}^{*}\right) \mathbb{E}\left(\operatorname{vec}\left(\underline{C}^{\mathrm{obs}}\right)\right) \mathbb{E}\left(\operatorname{vec}\left(\underline{C}^{\mathrm{obs}}\right)\right)^{*} \ell_{\Sigma} \\
= & \left(\ell^{*}-\ell_{\Sigma}^{*}\right) \Sigma \ell_{\Sigma} \\
= & \frac{1}{\|\operatorname{vec}(\underline{\mathcal{P}})\|_{\Sigma}^{2}}\left(\ell^{*}-\ell_{\Sigma}^{*}\right) \operatorname{vec}(\underline{\mathcal{P}})=0,
\end{aligned}
$$


where the last equality follows by the unit gain property (5.17). Hence we have

$$
\begin{aligned}
\operatorname{Var}\left(\ell^{*} \operatorname{vec}\left(\underline{C}^{\text {obs }}\right)\right) & =\operatorname{Var}\left(\ell_{\Sigma}^{*} \operatorname{vec}\left(\underline{C}^{\text {obs }}\right)\right)+\operatorname{Var}\left(\left(\ell^{*}-\ell_{\Sigma}^{*}\right) \operatorname{vec}\left(\underline{C}^{\text {obs }}\right)\right) \\
& =\operatorname{Var}\left(\ell_{\Sigma}^{*} \operatorname{vec}\left(\underline{C}^{\text {obs }}\right)\right)+\left(\ell^{*}-\ell_{\Sigma}^{*}\right) \Sigma\left(\ell-\ell_{\Sigma}\right)
\end{aligned}
$$

The last summand is always non-negative since $\Sigma$ is positive semi-definite. As $\Sigma$ is regular by assumption, it is even positive definite and hence the last summand vanishes if and only if $\ell=\ell_{\Sigma}$. As this case is excluded by assumption we conclude

$$
\begin{aligned}
\operatorname{Var}\left(\ell^{*} \operatorname{vec}\left(\underline{C}^{\text {obs }}\right)\right) & =\operatorname{Var}\left(\ell_{\Sigma}^{*} \operatorname{vec}\left(\underline{C}^{\text {obs }}\right)\right)+\operatorname{Var}\left(\left(\ell^{*}-\ell_{\Sigma}^{*}\right) \operatorname{vec}\left(\underline{C}^{\text {obs }}\right)\right) \\
& >\operatorname{Var}\left(\ell_{\Sigma}^{*} \operatorname{vec}\left(\underline{C}^{\text {obs }}\right)\right) .
\end{aligned}
$$

As already noted, the weighted beamformers $\underline{\mathcal{I}}_{W}$ all belong to the set $\mathcal{U}$. If we restrict $\mathcal{U}$ to the class of estimators that are characterized by a minimization problem on a Hilbert space (cf. (5.2)), we get a similar optimality result for the variance

$$
V_{W}:=\operatorname{Var} \underline{\mathcal{I}}_{W}=\mathbb{E}\left[\left|\underline{\mathcal{I}}_{W}-\mathbb{E}\left(\underline{\mathcal{I}}_{W}\right)\right|^{2}\right] .
$$

Corollary 5.6 (Variance optimality of iv-f [RSHE20, Theorem 4.1]).

Assume that the data covariance matrix $\Sigma=\operatorname{Cov}\left(\operatorname{vec}\left(\underline{C}^{\text {obs }}\right)\right)$ is regular, then for any Hermitian, positive definite matrix $W \in \mathbb{C}^{M^{2}}$, the variance of the corresponding beamformer $\underline{\underline{I}}_{W}$ is bounded from below by the variance of the iv-f beamformer i.e.

$$
\operatorname{Var}\left(\underline{\mathcal{I}}_{\Sigma}\right) \leq \operatorname{Var}\left(\underline{\mathcal{I}}_{W}\right)
$$

Proof. The statement follows directly from Theorem 5.5. A direct proof can be found in RSHE20, Theorem 4.1].

\subsection{Weighted DAMAS}

The principle of weighted data norms can also be transferred to the discrete DAMAS problem. The weighted version of the point spread function is defined by

$$
\underline{\psi}_{W}: \Omega \times \Omega \rightarrow \mathbb{C}, \quad\left(\mathbf{y}, \mathbf{y}^{\prime}\right) \mapsto \underset{\mu \in \mathbb{C}}{\operatorname{argmin}}\left\|\operatorname{vec}\left(\underline{\mathcal{P}_{\mathbf{y}^{\prime}}}\right)-\mu \operatorname{vec}\left(\underline{\mathcal{P}_{\mathbf{y}}}\right)\right\|_{W}^{2}
$$

For a convex penalty functional $\underline{\mathcal{R}}: \mathbb{R}^{N} \rightarrow \mathbb{R}$ and a regularization parameter $\alpha>0$, the corresponding regularized minimization problem is given by

$$
\min _{\underline{q} \in \mathbb{R}^{N}}\left\|H_{W} \underline{q}-\underline{y}_{W}^{\mathrm{bf}}\right\|_{2}^{2}+\alpha \underline{\mathcal{R}}(\underline{q})
$$


with

$$
\left[H_{W}\right]_{n l}=\psi_{W}\left(\mathbf{y}_{n}, \mathbf{y}_{l}\right) \text { and } \quad\left[\underline{y}_{W}^{\mathrm{bf}}\right]_{n}=\underline{\mathcal{I}}_{W}\left(\mathbf{y}_{n}\right) \text { for } n=1, \ldots, N
$$

\subsection{Weighted CMF}

The discrete CMF problem can be generalized in a similar way. As the discrete CMF forward operator maps from $\mathbb{R}^{N}$ to $\mathbb{C}^{n_{\text {mic }} \times n_{\text {mic }}}$, the Frobenius norm on the image space has to be replaced by the weighted norm induced by $W$. That yields the following regularized minimization problem

$$
\min _{\underline{q} \in \mathbb{R}^{N}}\left\|\operatorname{vec}\left(\underline{\mathcal{G}} \underline{M}_{q} \underline{\mathcal{G}}^{*}\right)-\operatorname{vec}\left(\underline{C}^{\mathrm{obs}}\right)\right\|_{W}^{2}+\alpha \underline{\mathcal{R}}(\underline{q}) .
$$

We recall that the discrete source multiplication operator was defined by $\underline{M_{q}}=\operatorname{diag}\left(q_{1}, \ldots, q_{N}\right)$ with

$$
q_{n}=\int_{\Omega_{n}} q(\mathbf{y}) d \mathbf{y}
$$

and the discrete volume potential operator by

$$
\underline{\mathcal{G}}_{m n}=g\left(\mathbf{x}_{m}, \mathbf{y}_{n}\right) \text {. }
$$




\section{Chapter}

\section{Computational Aspects}

In this chapter we investigate a procedure for the numerical solution of the discrete CMF and DAMAS problem. We discuss the most important aspects that have to be considered for an implementation of imaging methods and the evaluation of experimental data.

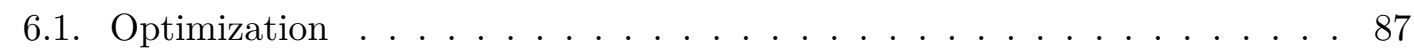

6.1.1. Fast Iterative Shrinkage-Thresholding Algorithm . . . . . . . . . . 91

6.1.2. Choice of the regularization parameters . . . . . . . . . . . . . 93

6.2. Discretization . . . . . . . . . . . . . . . . . . 94

\subsection{Optimization}

We will discuss optimization algorithms for Tikhonov functionals of the form

$$
\mathcal{J}(q)=\frac{1}{2}\left\|T q-y^{\mathrm{obs}}\right\|_{\mathbb{Y}}^{2}+\mathcal{R}(q) .
$$

Here $T: L^{2}(\Omega) \rightarrow \mathbb{Y}$ is a bounded linear operator between Hilbert spaces $L^{2}(\Omega)$ and $\mathbb{Y}$, and $\mathcal{R}: L^{2}(\Omega) \rightarrow[0, \infty]$ denotes the penalty functional. Note that both Tikhonov functionals (CMF and DAMAS) from 4.15) and 4.16) belong to this class (see Table 6.1). Since in aeroacoustic experiments the regions of large source power values are are usually relatively small compared to the entire source domain, the sparsity of the reconstructed source is often a reasonable assumption. Therefore, we will employ sparsity promoting penalty functionals. More precisely we will consider a penalty functional of the form

$$
\mathcal{R}: L^{2}(\Omega) \rightarrow[0, \infty] \quad \mathcal{R}(q)=\chi_{\mathcal{N}_{+}}(q)+\frac{\alpha_{2}}{2}\|q\|_{L^{2}(\Omega)}^{2}+\alpha_{1}\|q\|_{L^{1}(\Omega)},
$$




CMF DAMAS

\begin{tabular}{lll}
\hline Forward operator & $T=\mathcal{C}($ Proposition 4.1$)$ & $T=\mathcal{C}^{*} \mathcal{C}$ (Proposition 4.2 \\
Observed data & $y^{\text {obs }}=C^{\text {obs }}$ & $y^{\text {obs }}=\mathcal{C}^{*} C^{\text {obs }}$ \\
Data space & $\mathbb{Y}=\operatorname{HS}\left(L^{2}(\mathbb{M})\right)$ & $\mathbb{Y}=L^{2}(\Omega)$
\end{tabular}

Table 6.1.: Operators and data spaces for generalized Tikhonov regularization of CMF and $D A M A S$.

where $\mathcal{N}_{+}$is the set of a.e. nonnegative functions i.e.

$$
\mathcal{N}_{+}=\left\{f \in L^{2}(\Omega): f \geq 0 \text { a.e. }\right\}
$$

and $\chi_{\mathcal{N}_{+}}$its indicator function

$$
\chi_{\mathcal{N}_{+}}(q)=\left\{\begin{array}{ll}
0 & \text { if } q \in \mathcal{N}_{+} \\
\infty & \text { else }
\end{array} .\right.
$$

The first summand of the penalty functional 6.2 enforces a nonnegative solution. The $L^{2}$-norm penalty makes the Tikhonov functional stricly convex and therefore the minimizer is unique. The $L^{1}$-norm penalty on the other hand promotes a sparse solution which is often desirable (see i.e. [HS17, YLSC08, Suz08]).

Proposition 6.1 (Existence \& uniqueness for $L^{1}-L^{2}$ penalty).

Let $\alpha_{2}>0$, then the Tikhonov functional in (6.1) has a unique minimizer $\hat{q}_{\alpha_{1}, \alpha_{2}} \in L^{2}(\Omega)$.

Proof. We show that $\mathcal{R}$ is weakly sequentially lower semicompact and strictly convex (Assumption 4.13).

$\mathcal{R}$ is strictly convex since all summands are convex and $\alpha_{2}\|\cdot\|_{L^{2}(\Omega)}^{2}$ is strictly convex. Now let $\beta \geq 0$ and $\left(f_{n}\right)_{n \in \mathbb{N}} \subset \operatorname{lev}_{\beta}(\mathcal{R})$. This implies that $\left\|f_{n}\right\|_{L^{2}(\Omega)}$ is uniformly bounded since

$$
\left\|f_{n}\right\|_{L^{2}(\Omega)}^{2} \leq \frac{2}{\alpha_{2}} \mathcal{R}\left(f_{n}\right) \leq \frac{2}{\alpha_{2}} \beta
$$

Hence, there exists a subsequence $\left(f_{n_{k}}\right)_{k \in \mathbb{N}} \subset\left(f_{n}\right)_{n \in \mathbb{N}}$ that converges weakly to an element $f$ (see e.g. [BC17, Lemma 2.45, p.37]). Moreover, the sublevel sets $\operatorname{lev}_{\beta}(\mathcal{R})$ are convex and closed. Convexity follows by the definition of $\mathcal{R}$. To show closedness we take a sequence $\left(q_{n}\right)_{n \in \mathbb{N}} \subset \operatorname{lev}_{\beta}(\mathcal{R})$ that converges to some $q \in L^{2}(\Omega)$ and use the fact that $\Omega$ is bounded. This yields

$$
\begin{aligned}
\mathcal{R}(q) & \leq \mathcal{R}\left(q_{n}\right)+\alpha_{1}\left\|q-q_{n}\right\|_{L^{1}(\Omega)}+\frac{\alpha_{2}}{2}\left\|q-q_{n}\right\|_{L^{2}(\Omega)}^{2}+\alpha_{2}\left\|q_{n}\right\|_{L^{2}(\Omega)}\left\|q-q_{n}\right\|_{L^{2}(\Omega)} \\
& \leq \beta+\left(\alpha_{1} \sqrt{|\Omega|}+\frac{\alpha_{2}}{2}\left\|q-q_{n}\right\|_{L^{2}(\Omega)}+\alpha_{2}\left\|q_{n}\right\|_{L^{2}(\Omega)}\right)\left\|q-q_{n}\right\|_{L^{2}(\Omega)}
\end{aligned}
$$


The expression in the round brackets is uniformly bounded and the last factor converges to 0 . Thus for $n \rightarrow \infty$ we obtain $\mathcal{R}(q) \leq \beta$ i.e. $q \in \operatorname{lev}_{\beta}(\mathcal{R})$. Convex closed sets are also weakly sequentially closed (cf. [BC17, Theorem 3.34, p.60]) and therefore $\mathcal{R}$ is weakly sequentially lower semicontinuous. Hence we obtain

$$
\mathcal{R}(f) \leq \liminf \mathcal{R}\left(f_{n_{k}}\right) \leq \beta
$$

i.e. $f \in \operatorname{lev}_{\beta}(\mathcal{R})$. Thus $\mathcal{R}$ is weakly sequentially lower semicompact and the statement follows by Theorem 4.14 .

Finally we would like to prove convergence of the Tikhonov minimizers to the true solution $q \in L^{\infty}(\Omega)$ of the operator equation

$$
T q=y
$$

as the noise level tends to zero. For a generic convex penalization functional $\mathcal{R}$, standard convergence theorems in regularization theory (see e.g. $\left[\mathrm{SGG}^{+}\right.$09, $\mathbf{S K H K 1 2}$ ) yield convergence to the so-called $\mathcal{R}$-minimizing solution

$$
q^{\dagger}:=\underset{q \in L^{2}(\Omega) T q=y}{\operatorname{argmin}} \mathcal{R}(q) .
$$

Hence, in the convergence analysis we have to make sure that the $\mathcal{R}$-minimizing solution belongs to $L^{\infty}(\Omega)$. To this end, we define the following penalty functional for constants $U>0$ and $\theta \in[0,1]$

$$
\tilde{\mathcal{R}}_{\theta, U}: L^{2}(\Omega) \rightarrow[0, \infty] \quad \tilde{\mathcal{R}}_{\theta, U}=\chi_{\mathcal{N}_{+}^{U}}(q)+\frac{1-\theta}{2}\|q\|_{L^{2}(\Omega)}^{2}+\theta\|q\|_{L^{1}(\Omega)},
$$

where $\mathcal{N}_{+}^{U}$ is the set of a.e. nonnegative functions that are bounded by $U$ i.e.

$$
\mathcal{N}_{+}^{U}=\left\{f \in L^{2}(\Omega): f(\mathbf{x}) \in[0, U] \text { for a.e. } \mathbf{x} \in \Omega\right\} .
$$

For a deterministic noise model we get the following standard convergence theorem for the Tikhonov functional

$$
\tilde{\mathcal{J}}_{\alpha, y^{\mathrm{obs}}}(q)=\frac{1}{2}\left\|T q-y^{\mathrm{obs}}\right\|_{\mathbb{Y}}^{2}+\alpha \tilde{\mathcal{R}}_{\theta, U}(q) .
$$

Theorem 6.2 (Convergence of Tikhonov minimizers).

Let $T, \mathbb{Y}$ and $y^{\text {obs }}$ be defined according to Table 6.1 and consider an exact source power function $q \in L^{\infty}(\Omega)$ with exact data $y:=T q$. Moreover, let $\theta \in[0,1)$ and $U \geq\|q\|_{L^{\infty}(\Omega)}$. Assume that we have a sequence of noise levels $\left(\delta_{k}\right)_{k \in \mathbb{N}}$ converging to 0 and a sequence of data $\left(y_{k}^{\text {obs }}\right)_{k \in \mathbb{N}} \subset \mathbb{Y}$ with $\left\|y_{k}^{\text {obs }}-y\right\|_{\mathbb{Y}} \leq \delta_{k}$. Suppose that the sequence of regularization 
parameters $\left(\alpha_{k}\right)_{k \in \mathbb{N}}$ satisfies

$$
\lim _{k \rightarrow \infty} \alpha_{k}=0 \quad \text { and } \quad \lim _{k \rightarrow \infty} \frac{\delta_{k}^{2}}{\alpha_{k}}=0 .
$$

Then there exist unique Tikhonov minimizers $q_{k} \in L^{\infty}(\Omega)$ defined by

$$
q_{k}:=\underset{\tilde{q} \in L^{2}(\Omega)}{\operatorname{argmin}} \tilde{\mathcal{J}}_{\alpha_{k}, y_{k}^{\text {obs }}}(\tilde{q})
$$

and we have the following types of convergence

$$
\begin{aligned}
& q_{k} \rightarrow q \text { weakly in } L^{2}(\Omega) \\
& \tilde{\mathcal{R}}_{\theta, U}\left(q_{k}\right) \rightarrow \tilde{\mathcal{R}}_{\theta, U}(q) \\
& \lim _{k \rightarrow \infty}\left\|q_{k}-q\right\|_{L^{2}(\Omega)}=0
\end{aligned}
$$

Proof. Since $\theta \in[0,1)$ we can show existence and uniqueness of Tikhonov minimizers $q_{k}$ similar to Theorem 6.1. Moreover, the $\tilde{\mathcal{R}}_{\theta, U}$-minimizing solution

$$
q^{\dagger}=\underset{q \in L^{2}(\Omega) T q=y}{\operatorname{argmin}} \tilde{\mathcal{R}}_{\theta, U}(q)
$$

is unique and $q=q^{\dagger}$ as $T$ is injective on $L^{\infty}(\Omega)$ (see Theorem 3.11 and Corollary 4.11). Now the first two convergence properties $6.5 \mathrm{a}$ and $6.5 \mathrm{~b}$ follow by standard results in regularization theory (see e.g. $\left[\mathrm{SGG}^{+} 09\right.$, Theorem 3.26, p.66]). For the proof of strong convergence we consider the two sequences

$$
a_{k}:=\tilde{\mathcal{R}}_{\theta, U}\left(q_{k}\right)-\tilde{\mathcal{R}}_{\theta, U}(q) \quad \text { and } \quad b_{k}:=\left\langle q+\theta, q_{k}-q\right\rangle_{L^{2}(\Omega)} .
$$

By the result above, each sequence converges to 0 and hence also $\left(a_{k}-b_{k}\right) \rightarrow 0$. Computing the difference explicitly we obtain

$$
\begin{aligned}
& a_{k}-b_{k} \\
= & \frac{(1-\theta)}{2}\left\|q_{k}\right\|_{L^{2}(\Omega)}^{2}+\theta\left\|q_{k}\right\|_{L^{1}(\Omega)}-\frac{(1-\theta)}{2}\|q\|_{L^{2}(\Omega)}^{2}-\theta\|q\|_{L^{1}(\Omega)} \\
& -\left\langle q, q_{k}\right\rangle_{L^{2}(\Omega)}+\|q\|_{L^{2}(\Omega)}^{2}-\theta\left\|q_{k}\right\|_{L^{1}(\Omega)}+\theta\|q\|_{L^{1}(\Omega)} \\
= & \frac{(1-\theta)}{2}\left\|q_{k}\right\|_{L^{2}(\Omega)}^{2}+\frac{(1-\theta)}{2}\|q\|_{L^{2}(\Omega)}^{2}-(1-\theta)\left\langle q, q_{k}\right\rangle_{L^{2}(\Omega)}+\theta\|q\|_{L^{2}(\Omega)}^{2}-\theta\left\langle q, q_{k}\right\rangle_{L^{2}(\Omega)} \\
= & \frac{(1-\theta)}{2}\left\|q-q_{k}\right\|_{L^{2}(\Omega)}^{2}+\theta\|q\|_{L^{2}(\Omega)}^{2}-\theta\left\langle q, q_{k}\right\rangle_{L^{2}(\Omega)} \\
= & \frac{(1-\theta)}{2}\left\|q-q_{k}\right\|_{L^{2}(\Omega)}^{2}+\theta\left\langle q, q-q_{k}\right\rangle_{L^{2}(\Omega)} .
\end{aligned}
$$

The second summand of the last line converges to 0 due to weak convergence (6.5a). As the entire sum in the last line converges to 0 as well, we conclude that the first summand 
$\left(\frac{(1-\theta)}{2}\left\|q-q_{k}\right\|_{L^{2}(\Omega)}^{2}\right)$ must also converge to 0 . This proves strong convergence in $L^{2}(\Omega)$ $6.5 \mathrm{c}$.

Note that for practical applications we can choose the upper bound $U \in \mathbb{R}$ sufficiently large such that it can be neglected for the numerical treatment and we can work with the penalty functional defined in 6.2 .

\subsubsection{Fast Iterative Shrinkage-Thresholding Algorithm}

The fast iterative shrinkage-thresholding algorithm (FISTA) for a $\ell^{1}$-norm penalty was first proposed by Beck \& Teboulle BT09. The FISTA algorithm makes use of the proximal mapping of the penalty functional $\mathcal{R}$. In our $L^{2}$ setting and with $\tau>0$ it is defined as

$$
\operatorname{prox}_{\tau \mathcal{R}}: L^{2}(\Omega) \rightarrow L^{2}(\Omega) \quad \operatorname{prox}_{\tau \mathcal{R}}(g)=\underset{f \in L^{2}(\Omega)}{\operatorname{argmin}}\left[\mathcal{R}(f)+\frac{1}{2 \tau}\|f-g\|_{L^{2}(\Omega)}^{2}\right] .
$$

The proximal mapping of the discussed $L^{1}-L^{2}$ penalty with nonnegativity constraint 6.2 is given by

$$
\begin{aligned}
\operatorname{prox}_{\tau \mathcal{R}}(g) & =\underset{f \in L^{2}(\Omega)}{\operatorname{argmin}}\left[\mathcal{R}(f)+\frac{1}{2 \tau}\|f-g\|_{L^{2}(\Omega)}^{2}\right] \\
& =\underset{f \in \mathcal{N}_{+}}{\operatorname{argmin}}\left[\frac{\alpha_{2}}{2}\|f\|_{L^{2}(\Omega)}^{2}+\alpha_{1}\|f\|_{L^{1}(\Omega)}+\frac{1}{2 \tau}\|f-g\|_{L^{2}(\Omega)}^{2}\right] \\
& =\underset{f \in \mathcal{N}_{+}}{\operatorname{argmin}}\left[\frac{\alpha_{2} \tau+1}{2 \tau} \int_{\Omega} f^{2}-\frac{1}{\tau} \int_{\Omega} f\left(g-\tau \alpha_{1}\right)\right] \\
& =\underset{f \in \mathcal{N}_{+}}{\operatorname{argmin}}\left[\int_{\Omega} f^{2}-2 \int_{\Omega} f\left(\frac{g-\tau \alpha_{1}}{\alpha_{2} \tau+1}\right)\right] \\
& =\underset{f \in \mathcal{N}_{+}}{\operatorname{argmin}}\left\|f-\left(\frac{g-\tau \alpha_{1}}{\alpha_{2} \tau+1}\right)\right\|_{L^{2}(\Omega)}^{2} \\
& =P_{\mathcal{N}_{+}}\left(\frac{g-\tau \alpha_{1}}{\alpha_{2} \tau+1}\right) .
\end{aligned}
$$

Here $P_{\mathcal{N}_{+}}$denotes the $L^{2}$ metric projection onto $\mathcal{N}_{+}$i.e.

$$
\left(P_{\mathcal{N}_{+}}(f)\right)(x)=\max \{0, \operatorname{Re}(f(x))\} .
$$

A generic FISTA algorithm for Tikhonov functionals as in Equation 6.1) endowed with the discussed penalty functional 6.2 is presented in Algorithm 1 (cf. Bec17, p. 291] for a more general formulation). The algorithm approximates the unique Tikhonov minimizer given by

$$
q_{\left(\alpha_{1}, \alpha_{2}\right)}=\underset{q \in L^{2}(\Omega)}{\operatorname{argmin}} \frac{1}{2}\left\|T q-y^{\mathrm{obs}}\right\|_{\mathbb{Y}}^{2}+\chi_{A}(q)+\frac{\alpha_{2}}{2}\|q\|_{L^{2}(\Omega)}^{2}+\alpha_{1}\|q\|_{L^{1}(\Omega)} .
$$


The convergence of Algorithm 1 is ensured by the next proposition.

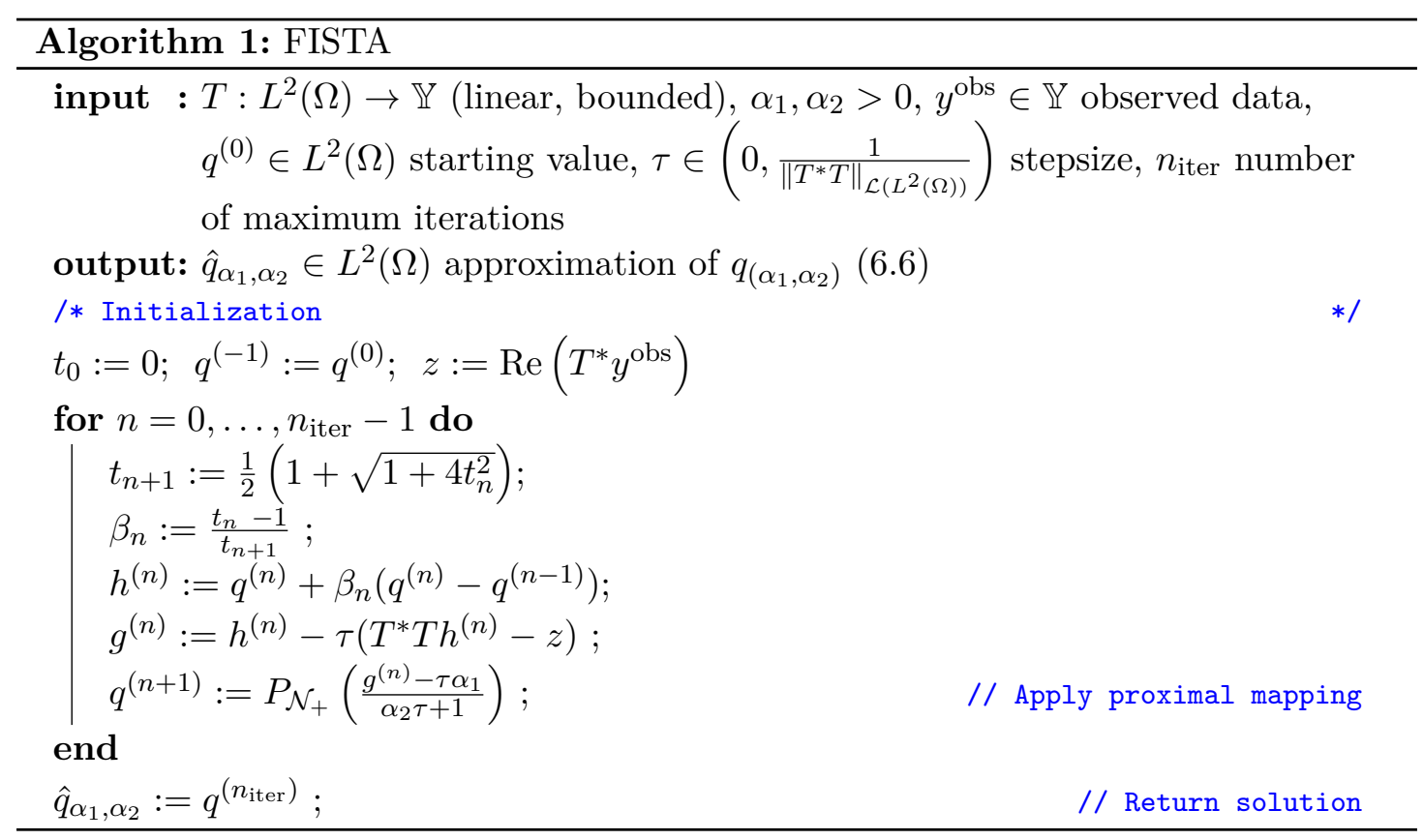


Proposition 6.3 (Convergence of FISTA).

Assume that $\operatorname{argmin} \mathcal{J}$ is non-empty, then for any minimizer $q^{\star} \in \operatorname{argmin} \mathcal{J}$ and $n \geq 1$

$$
\mathcal{J}\left(q^{(n)}\right)-\mathcal{J}\left(q^{\star}\right) \leq \frac{2}{(n+1)^{2}}\left\|T^{*} T\right\|_{\mathcal{L}\left(L^{2}(\Omega)\right)}
$$

Proof. The penalty functional $\mathcal{R}$ is proper, convex and lower semicontinuous by definition. Furthermore the gradient of $F(q)=\frac{1}{2}\left\|T q-y^{\text {obs }}\right\|_{L^{2}(\Omega)}^{2}$ is Lipschitz continuous with Lipschitz constant $\left\|T^{*} T\right\|_{\mathcal{L}\left(L^{2}(\Omega)\right)}$. Now the statement follows by the convergence properties of the generic FISTA algorithm [Bec17, Theorem 10.34, p.292].

\subsubsection{Choice of the regularization parameters}

For a practical use, the choice of regularization parameters is of great importance. Therefore we will briefly discuss some strategies.

\section{Reduction to a single parameter:}

We may set

$$
\alpha_{1}=\theta \alpha \quad \alpha_{2}=(1-\theta) \alpha
$$

for $\theta \in[0,1]$ and $\alpha>0$. This principle has been proposed e.g. in Zou \& Hastie ZH05 and is often referred to as elastic net regularization. The parameter $\theta$ models the preference towards the $L^{1}$ or $L^{2}$ penalty and the parameter $\alpha$ controls the magnitude of the entire regularization term. In practice one may choose $\theta$ a-priori and then use a common parameter choice rule for problems with a single regularization parameter.

\section{Discrepancy principle:}

One of the most common parameter choice rules is Morozov's discrepancy principle [Mor66]. It requires a measure of the noise level on the data i.e. for deterministic noise a bound $\delta$ such that

$$
\left\|y-y^{\mathrm{obs}}\right\|_{\mathbb{Y}} \leq \delta
$$

where $y$ denotes the exact data. The discrepancy principle relies on the idea that for an approximate solution $\hat{q}_{\alpha}$ one should choose $\alpha$ such that $\left\|T \hat{q}_{\alpha}-y^{\text {obs }}\right\|_{\mathbb{Y}} \approx \delta$. More explicitly one chooses $\alpha=\alpha_{\delta}$ such that

$$
\alpha_{\delta}=\sup \left\{\alpha>0:\left\|T \hat{q}_{\alpha}-y^{\text {obs }}\right\|_{\mathbb{Y}} \leq \tau \delta\right\}
$$

with some constant $\tau>1$ (cf. [EHN96, Section 4.3.]). A measure of the noise level in case of random noise may be the provided by the statistics of the correlation data (cf. Equation (4.37) and Section 4.3.2). However, for random noise the discrepancy principle (6.7) is not an optimal strategy since it tends to overestimate the regularization 
parameter for fine discretizations (i.e. large $N$ ). This phenomenon has been investigated e.g. in [Han10, Chapter 5].

\section{Lepskiĩ principle:}

This parameter choice rule goes back to the work of Lepskiu [Lep90. Let $q$ denote the exact solution of the inverse problem and $q_{\alpha}, \hat{q}_{\alpha}$ the Tikhonov solution for regularization parameter $\alpha$ for the exact resp. noisy data. The Lepskil principle is based on the error decomposition

$$
\left\|q-\hat{q}_{\alpha}\right\| \leq\left\|q-q_{\alpha}\right\|+\left\|q_{\alpha}-\hat{q}_{\alpha}\right\|
$$

The first term of the upper bound is deterministic, unknown and expected to be an increasing function of alpha. The second term depends on the data noise and is expected to be an decreasing function of alpha. Moreover in many scenarios there are good upper bounds available for the propagated data noise error $\left\|q_{\alpha}-\hat{q}_{\alpha}\right\|$. In order to implement the Lepskil principle, we need a finite set of regularization parameters $\alpha_{1}<\alpha_{2}<\cdots<$ $\alpha_{m}$ and an upper bound on the propagated data noise error, given by a decreasing function $\Psi:\{1, \ldots, m\} \rightarrow \mathbb{R}^{+}$. Within this thesis we will follow the general approach of the Lepskil principle for random noise that was presented in [MP06]. Applying these ideas to the scenario within this thesis we get the following procedure:

For discrete Tikhonov estimators $\underline{q}_{\alpha}, \underline{\hat{q}}_{\alpha}$, assume that there is a decreasing function $\Psi$ such that for all $j=1, \ldots, m$ holds

$$
\mathbb{E}\left(\left\|\underline{q}_{\alpha_{j}}-\underline{\hat{q}}_{\alpha_{j}}\right\|_{2}^{2}\right) \leq \Psi^{2}(j) .
$$

Now we choose the regularization parameter $\alpha_{j \text { Lep }}$ with index

$$
j^{\text {Lep }}:=\max \left\{j:\left\|\underline{\hat{q}}_{\alpha_{i}}-\underline{\hat{q}}_{\alpha_{j}}\right\|_{2} \leq 4 \Psi(i) \text { for all } i \leq j\right\} .
$$

For more theoretical analysis of this parameter choice rule (e.g. an abstract oracle inequality) we refer to [MP06. In our setup, the upper bound $\Psi$ cannot be computed analytically but since we have an estimate of the covariance matrix of the data noise (cf. Section 4.3), $\Psi$ can be estimated by a Monte Carlo simulation (see next chapter).

\subsection{Discretization}

Recall that $n_{\text {mic }}$ denotes the number of microphones in the array, $N$ the number of focus points and $\underline{C}^{\text {obs }} \in \mathbb{C}^{n_{\text {mic }} \times n_{\text {mic }}}$ the CSM. Similar to Chapter 5 , we will consider vectorized data.

\section{Subset of sensor pairings}

In practice it is often beneficial for the reconstruction results to consider only a subset 
$\mathcal{S}$ of all possible sensor pairings (see e.g. [Sij04])

$$
\mathcal{S} \subseteq\left\{1, \ldots, n_{\text {mic }}\right\}^{2} .
$$

From the vectorized correlation data vec $\left(\underline{C}^{\text {obs }}\right)$, all entries are removed that do not correspond to a sensor pairing from the set $\mathcal{S}$. In many experiments, the diagonal entries of the CSM is corrupted by strong noise e.g. by the turbulent boundary in closed wind tunnel test sections. In such scenarios one may set

$$
\mathcal{S}=\left\{(m, l) \in\left\{1, \ldots, n_{\text {mic }}\right\}^{2}: m \neq l\right\},
$$

which is known as diagonal removal. Since the CSM is Hermitian, the data can be further reduced by considering only the lower triangular part with or without the diagonal i.e.

$$
\mathcal{S}=\left\{(m, l) \in\left\{1, \ldots, n_{\mathrm{mic}}\right\}^{2}: m \leq l\right\} \quad \text { or } \mathcal{S}=\left\{(m, l) \in\left\{1, \ldots, n_{\mathrm{mic}}\right\}^{2}: m<l\right\}
$$

Following the setup described above, we denote by $y^{\text {cor }} \in \mathbb{C}^{n_{\text {cor }}}$ the corresponding correlation vector, where $n_{\text {cor }}:=|\mathcal{S}|$. To incorporate the framework from Chapter 5 on weighted imaging methods, we denote by $W_{\mathcal{S}} \in \mathbb{C}^{n_{\text {cor }} \times n_{\text {cor }}}$ the weighting matrix and by $\operatorname{vec}_{\mathcal{S}}\left(\mathcal{P}_{\mathbf{y}_{n}}\right) \in \mathbb{C}^{n_{\text {cor }}}$ the monopole correlations that are obtained by only considering the sensor pairings in $\mathcal{S}$. With this notation the Beamforming result at a focus point $\mathbf{y}_{n}$ is given by

$$
\underline{\mathcal{I}}_{W_{\mathcal{S}}}\left(\mathbf{y}_{n}\right)=\frac{\underline{y}^{\operatorname{cor} *} W_{\mathcal{S}}{ }^{-1} \operatorname{vec}_{\mathcal{S}}\left(\mathcal{P}_{\mathbf{y}_{n}}\right)}{\operatorname{vec}\left(\mathcal{P}_{\mathbf{y}_{n}}\right)^{*} W_{\mathcal{S}}{ }^{-1} \operatorname{vec}_{\mathcal{S}}\left(\mathcal{P}_{\mathbf{y}_{n}}\right)}
$$

The vector of Beamforming results is then defined as

$$
\underline{y}^{\mathrm{bf}} \in \mathbb{C}^{N} \quad \underline{y}_{n}^{\mathrm{bf}}=\underline{\mathcal{I}}_{W_{\mathcal{S}}}\left(\mathbf{y}_{n}\right) \text { for } n=1, \ldots, N .
$$

Finally we can define the discrete forward operator of the CMF problem

$$
\underline{T}^{\mathrm{CMF}}: \mathbb{R}^{N} \rightarrow \mathbb{C}^{n_{\mathrm{cor}}}: \quad \underline{q} \mapsto \operatorname{vec}_{\mathcal{S}}(\underline{\mathcal{C}}(\underline{q}))
$$

and the DAMAS problem

$$
\underline{T}^{\text {DAMAS }}: \mathbb{R}^{N} \rightarrow \mathbb{C}^{N}: \quad \underline{q} \mapsto\left[\frac{\left(\underline{T}^{\mathrm{CMF}} \underline{q}\right)^{*} W_{\mathcal{S}}^{-1} \operatorname{vec}_{\mathcal{S}}\left(\mathcal{P}_{\mathbf{y}_{n}}\right)}{\operatorname{vec}_{\mathcal{S}}\left(\mathcal{P}_{\mathbf{y}_{n}}\right)^{*} W_{\mathcal{S}}^{-1} \operatorname{vec}_{\mathcal{S}}\left(\mathcal{P}_{\mathbf{y}_{n}}\right)}\right]_{n=1}^{N}
$$

Note that we will use the scaled version of the normal equation for the discrete DAMAS problem as it was originally proposed by Brooks \& Humphreys [BH06]. 


\section{Discrete Tikhonov functionals}

The configurations for the discrete CMF and DAMAS problem are summarized in Table 6.2

\begin{tabular}{lll} 
& CMF & DAMAS \\
\hline Forward operator & $\underline{T}=\underline{T}^{\mathrm{CMF}} \sqrt{6.9)}$ & $\underline{T}=\underline{T}^{\mathrm{DAMAS}} \sqrt{6.10)}$ \\
Observed data & $\underline{y}^{\mathrm{obs}}=\underline{y}^{\mathrm{cor}}$ & $\underline{y}^{\mathrm{obs}}=\underline{y}^{\mathrm{bf}}$ \\
Data space & $\underline{\mathbb{Y}}=\mathbb{C}^{n_{\mathrm{cor}}}$ & $\underline{\mathbb{Y}}=\mathbb{C}^{N}$ \\
Data norm & $\|\cdot\|_{\underline{\mathbb{Y}}}=\|\cdot\|_{W_{\mathcal{S}}}$ & $\|\cdot\|_{\underline{\mathbb{Y}}}=\|\cdot\|_{2}$ \\
\hline
\end{tabular}

Table 6.2.: Configuration for the implementation of CMF and DAMAS.

That yields the following discretized version of the infinite dimensional Tikhonov functional (see 4.15 and 4.16)

$$
\underline{\mathcal{J}}(\underline{q})=\frac{1}{2}\left\|\underline{T} \underline{q}-y^{\mathrm{obs}}\right\|_{\underline{\mathbb{Y}}}^{2}+\alpha_{1}\|\underline{q}\|_{1}+\frac{\alpha_{2}}{2}\|\underline{q}\|_{2}^{2}+\underline{\mathcal{X}_{\mathcal{N}_{+}}}(\underline{q}),
$$

with

$$
\underline{\mathcal{X}_{\mathcal{N}_{+}}}(\underline{q})= \begin{cases}0 & \text { if } \forall n: q_{n} \geq 0 \\ \infty & \text { else }\end{cases}
$$




\section{Chapter}

\section{Numerical Experiments}

This chapter is intended to illustrate the concepts and numerical methods introduced in Chapter 45 and 6. In particular, we focus on aspects that are important from an experimenter's point of view. For each considered aspect a small computational example on a simple synthetic dataset is examined. By the end of the chapter, we will also show some results of source power reconstruction methods on an experimental dataset.

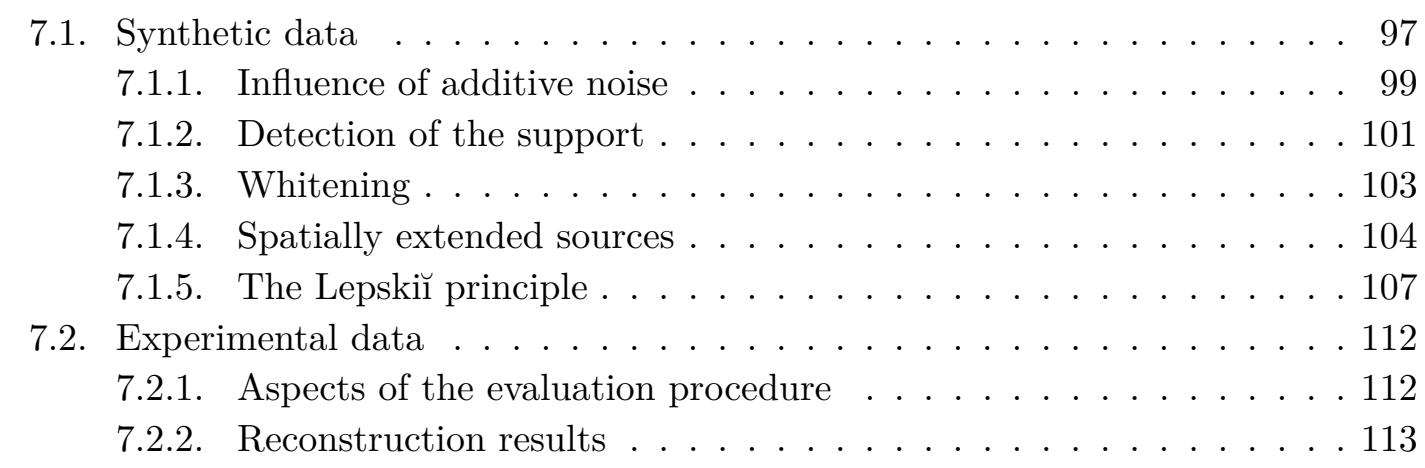

\subsection{Synthetic data}

All the numerical evaluations within this section are performed on a synthetic dataset whose framework is described in the following and illustrated in Figure 7.1.

Geometry and Propagation: Free field propagation model in $\mathbb{R}^{2}$ (i.e. Greens's function according to Equation (2.20) ) and a square source domain $\Omega=[-0.5 \mathrm{~m}, 0.5 \mathrm{~m}]^{2}$. The mach vector is set to $(0.2,0)^{\top}$ and the speed of sound to $c=343 \frac{\mathrm{m}}{\mathrm{s}}$. 
Discretization: The source domain is discretized by a equidistant grid with resolution $\Delta_{x y}$. To prevent inverse crimes, the data is generated on a finer grid with $\Delta_{x y}=0.005 \mathrm{~m}$ and the reconstruction is performed on a coarser grid with $\Delta_{x y}=0.02 \mathrm{~m}$.

Source power function: Sum of four approximate point sources, where each point source is supported on a square $R_{s}:=\left\{\mathbf{y}:\left|\mathbf{y}-\mathbf{y}_{s}\right|_{\infty} \leq 0.02\right\}$ with center $\mathbf{y}_{s}$ and side length 0.04 , for $s=1, \ldots, 4$. More precisely we set

$$
\begin{aligned}
& q(\mathbf{y})=\sum_{s=1}^{4} \mu_{s} 1_{R_{s}}(\mathbf{y}) \\
& \mathbf{y}_{1}=(-0.28,-0.08)^{\top}, \mathbf{y}_{2}=(0.02,0.34)^{\top}, \mathbf{y}_{3}=(0.12,0.24)^{\top}, \mathbf{y}_{4}=(0.32,-0.28)^{\top} \\
& \mu_{1}=10^{4}, \mu_{2}=7 \cdot 10^{3}, \mu_{3}=4 \cdot 10^{3}, \mu_{4}=7 \cdot 10^{3}
\end{aligned}
$$

Array: $n_{\text {mic }}=25$ Microphones arranged in a half circle with radius $0.75 \mathrm{~m}$ centered at the origin.

Data generation: For the finer grid for the data generation we have $N=4 \cdot 10^{4}$ grid points and the true source power vector is denoted by $\underline{q} \in \mathbb{R}^{N}$. To generate a datapoint we fix a frequency $f$ (and hence $k=\frac{2 \pi f}{c}$ ) and generate $n_{\text {samp }} \in \mathbb{N}$ pressure samples by

$$
\underline{p}^{(j)}=\underline{\mathcal{G}}\left(\eta^{(j)} \odot \sqrt{\underline{q}}\right)+\epsilon^{(j)} \quad \text { for } j=1, \ldots, n_{\text {samp }} .
$$

Here $\odot$ denotes the pointwise multiplication (Hadamard product) and $\eta^{(j)}, \epsilon^{(j)}$ are each iid samples from complex, vector-valued random variables $\eta$ resp. $\epsilon$ with

$$
\begin{aligned}
\eta & \sim\left[\mathcal{N}_{\mathbb{C}}(0,1)\right]^{N} \\
\epsilon & \sim\left[\mathcal{N}_{\mathbb{C}}\left(0, \rho_{\text {abs }}^{2}\right)\right]^{n_{\text {mic }}} \quad \rho_{\text {abs }}=\rho_{\text {rel }} \sqrt{\sum_{n=1}^{N}\left|q_{n}\right|} . \\
\eta & \perp \epsilon .
\end{aligned}
$$

The additive noise $\epsilon^{(j)}$ models additional measurement noise that may be caused for instance by the turbulent boundary layer in a windtunnel experiment. The empirical CSM estimate is then obtained by

$$
\underline{C}^{\mathrm{obs}}=\frac{1}{n_{\mathrm{samp}}} \sum_{j=1}^{n_{\mathrm{samp}}} \underline{p}^{(j)} \underline{p}^{(j)^{*}} .
$$

For the experiments we will vary the Parameters $f$ (frequency), $\rho_{\text {rel }}$ (additive noise level) and $n_{\text {samp }}$ (number of pressure samples). 


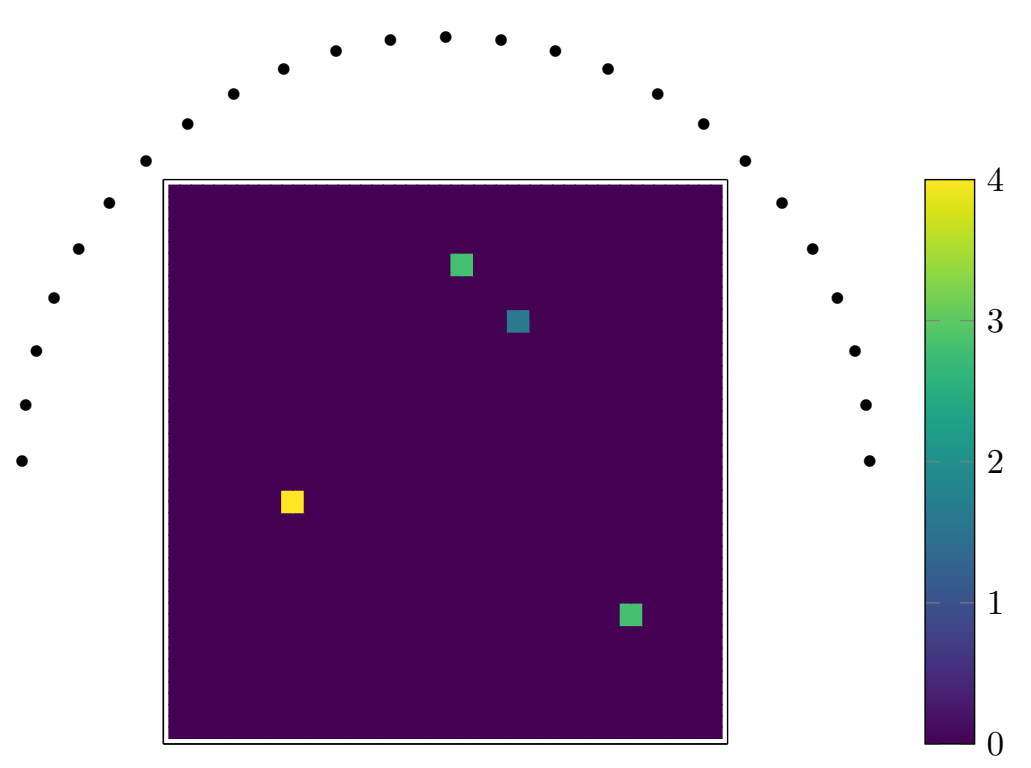

Figure 7.1.: Source and array configuration for the numerical experiment.

\subsubsection{Influence of additive noise}

Letting the number of pressure samples in 7.2 tend to infinity we observe that the empirical correlation matrix converges to

$$
\underline{C}+\rho_{\mathrm{abs}} I,
$$

where $\underline{C}=\mathbb{E}\left(\underline{\hat{p}}^{*}\right)$ denotes the exact correlation matrix of the microphone array. Hence, for large numbers of pressure samples and large additive noise levels, one expects that the diagonal entries of the empirical correlation matrix are much more corrupted by noise effects that the off-diagonal entries. This effect is well-known e.g. from measurements in closed wind tunnel test sections. In such cases one may consider only the off-diagonal entries of the empirical correlation matrix for the source power reconstruction as indicated in Section 6.2. Within this Section we will illustrate how the imaging result may be affected by strong noise on the diagonal and how diagonal removal may improve the imaging result.

Three imaging methods are evaluated: Conventional Beamforming and Tikhonov regularized CMF and DAMAS with penalty terms given in 6.11. For the latter two methods, FISTA (Algorithm 1) was employed using the following configuration.

- Fixed numer of iterations $n_{\text {iter }}=2000$.

- $\tau=0.95 \cdot \frac{1}{\lambda_{\max }}$, where $\frac{1}{\lambda_{\max }}$ denotes the largest eigenvalue of $\underline{T}^{*} \underline{T}$ (see Table 6.2 for the definition of $T$ ).

- Purely $L^{2}$ regularization i.e. $\theta=0$. 
- The reconstruction is evaluated for the regularization parameters $\alpha \in\left\{10^{-15+j}: j=0, \cdots, 15\right\}$.

Among the reconstructed source power vectors $\underline{\hat{q}}_{\alpha}$ we choose the solution with the lowest reconstruction error

$$
\mathcal{E}_{2}:=\left\|\underline{\hat{q}}_{\alpha}-\underline{q}_{2}\right\|
$$

where $q$ denotes the true source power vector on the reconstruction grid. Note that the $n$-th component of $\underline{q}$ is given by $\int_{\Omega_{n}} q(\mathbf{y}) d \mathbf{y}$.

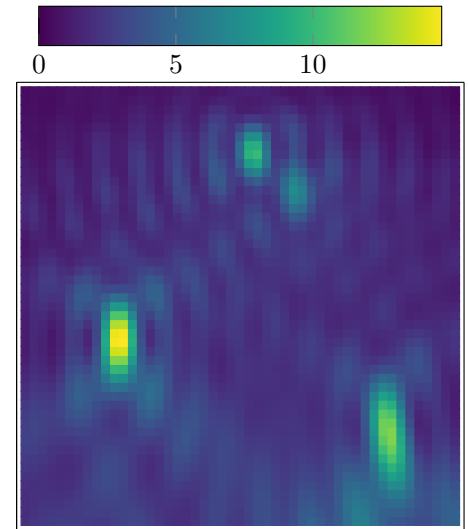

(a) $C B F$

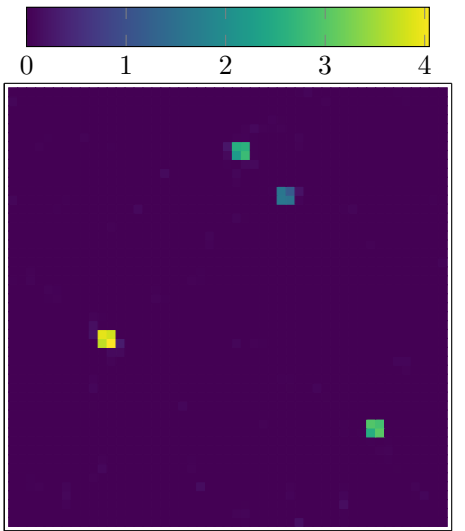

(b) DAMAS: $\mathcal{E}_{2}=1.20$

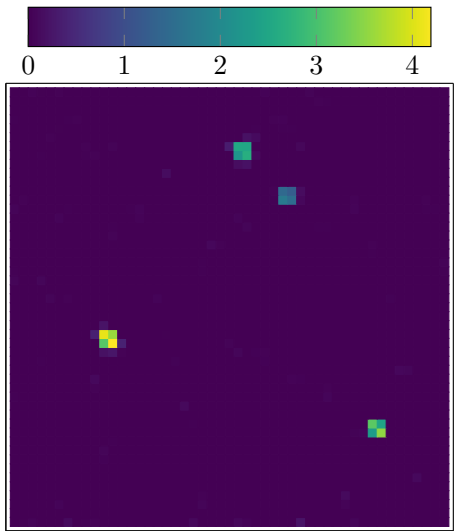

(c) $C M F: \mathcal{E}_{2}=1.59$

Figure 7.2.: Imaging results for $f=2000 \mathrm{~Hz}, n_{\mathrm{samp}}=1000, \rho_{\mathrm{rel}}=0.01$. Full CSM used.

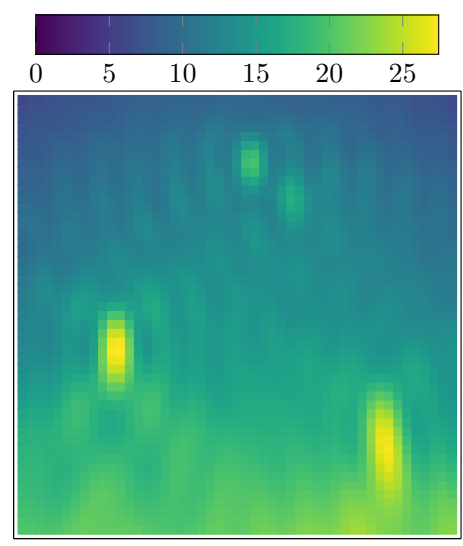

(a) $C B F$

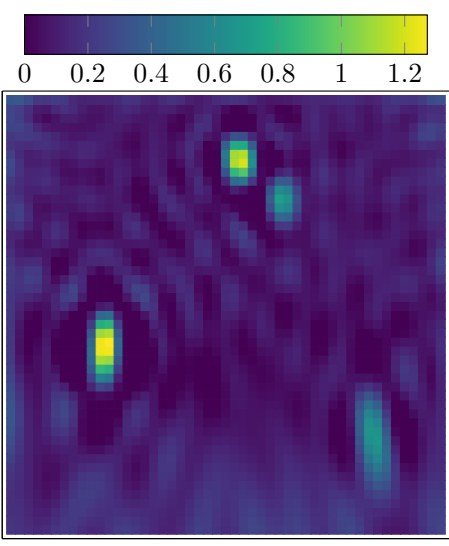

(b) DAMAS: $\mathcal{E}_{2}=11.11$

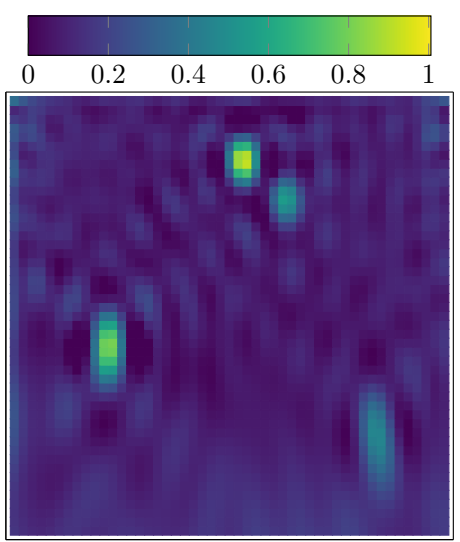

(c) $C M F: \mathcal{E}_{2}=11.36$

Figure 7.3.: Imaging results for $f=2000 \mathrm{~Hz}, n_{\text {samp }}=5000, \rho_{\text {rel }}=0.1$. Full CSM used. 


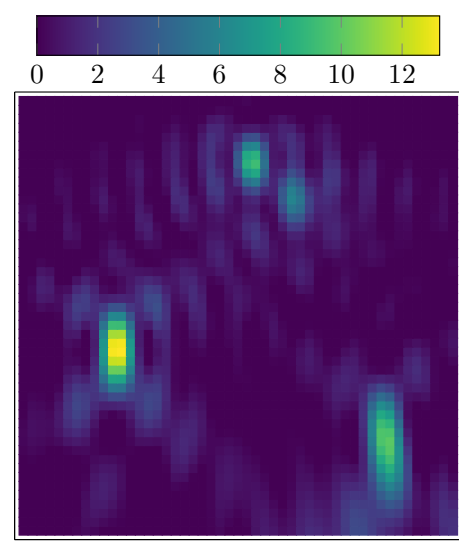

(a) $C B F$

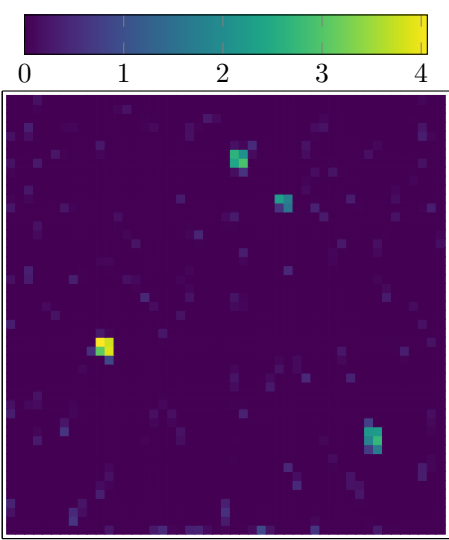

(b) DAMAS: $\mathcal{E}_{2}=4.77$

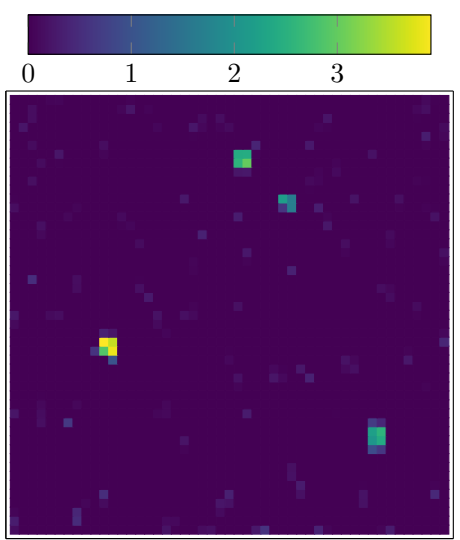

(c) $C M F: \mathcal{E}_{2}=3.70$

Figure 7.4.: Imaging results for $f=2000 \mathrm{~Hz}, n_{\mathrm{samp}}=5000, \rho_{\mathrm{rel}}=0.1$. Only off-diagonal parts of CSM used.

The evaluations show that in case of a low additive noise level (Figure 7.2), the reconstruction based on the full empirical correlation matrix works very well and yields results with low errors. However, when the number of averages and the additive noise level is increased the results suffer from noise effects and the imaging result is strongly blurred. In that case, the reconstruction based only on the off-diagonal entries of $\underline{C}^{\text {obs }}$ provides more accurate results. The $L^{2}$ error is smaller and the point sources are localized much better. Furthermore, noise effects are strongly reduced in regions where the true source is zero.

\subsubsection{Detection of the support}

Within this section we examine the influence of data noise on the factorization method from Section 4.2, which has been shown to be closely related to Capon's method. For our dataset, the observed covariance matrix $\underline{C}^{\text {obs }}$ will be always positive definite. Therefore, we can consider the discrete imaging functional given by (cf. 4.26)

$$
\underline{\mathcal{I}(\mathbf{y})}=\left(\underline{g}\left(\mathbf{y}_{0}\right)^{*} \underline{C}^{\mathrm{obs}^{-1}} \underline{g}\left(\mathbf{y}_{0}\right)\right)^{-1} .
$$

Figure 7.5 shows that for this simple source power distribution the reconstruction of the support works almost perfectly on exact data. However, for noisy data with $n_{\text {samp }}=100$ averages the accuracy drops significantly and gets much worse with increasing noise level. For relative additive noise levels of 0.05 and 0.1 , the values of the imaging functional are almost nowhere close to zero but the source locations may still be indicated by a local peak of the imaging functional. With increasing noise, the factorization method provides qualitatively similar results as Conventional Beamforming (cf. Figure 7.6). 


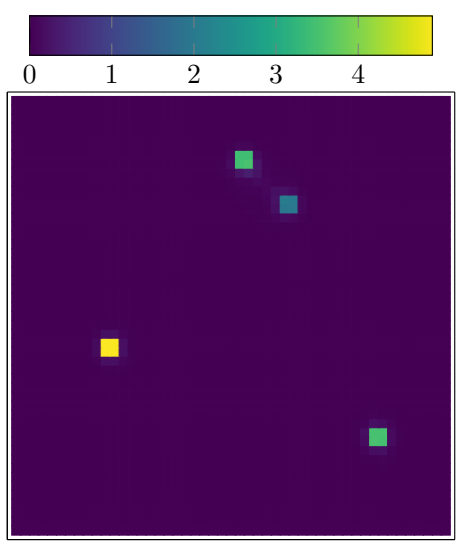

(a) Exact data.

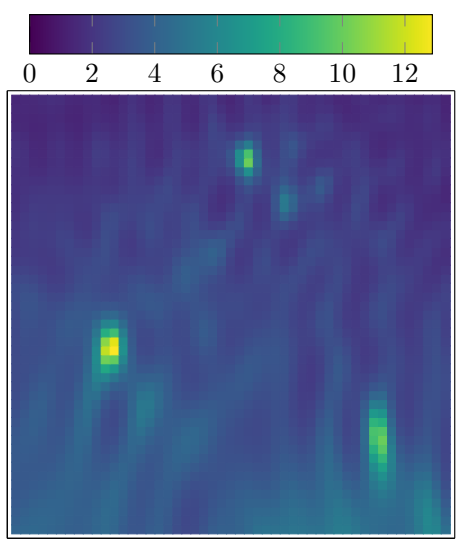

(c) $n_{\mathrm{samp}}=100, \rho_{\mathrm{rel}}=0.05$.

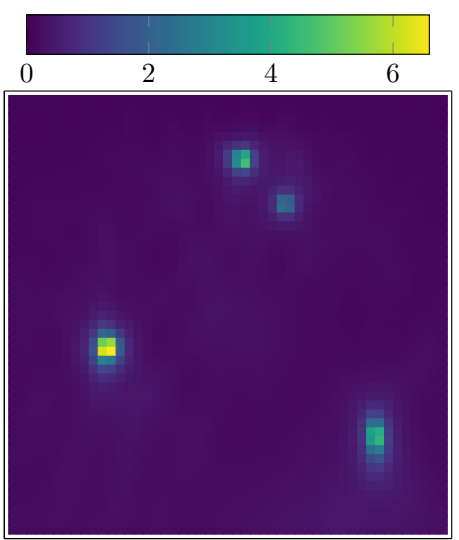

(b) $n_{\text {samp }}=100, \rho_{\text {rel }}=0.01$.

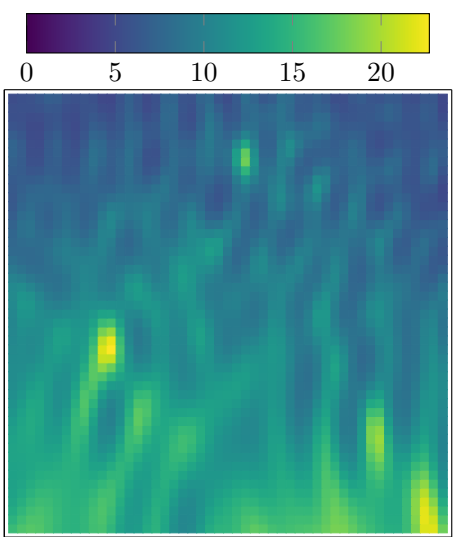

(d) $n_{\text {samp }}=100, \rho_{\text {rel }}=0.1$.

Figure 7.5.: Imaging result of the factorization method for $f=2000 \mathrm{~Hz}$.

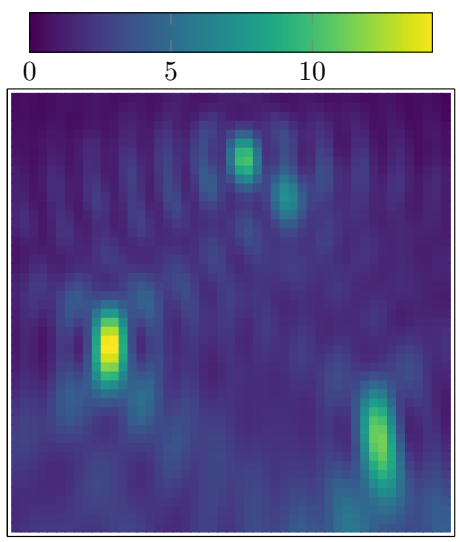

(a) Exact data.

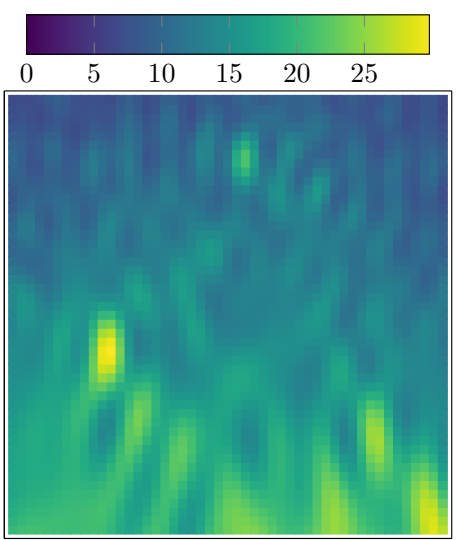

(b) $n_{\text {samp }}=100, \rho_{\text {rel }}=0.1$.

Figure 7.6.: Imaging result of Conventional Beamforming for $f=2000 \mathrm{~Hz}$. 


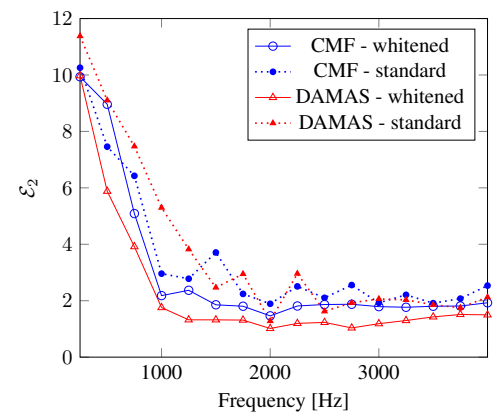

(a) $n_{\text {samp }}=1000, \rho_{\text {rel }}=0.01$.

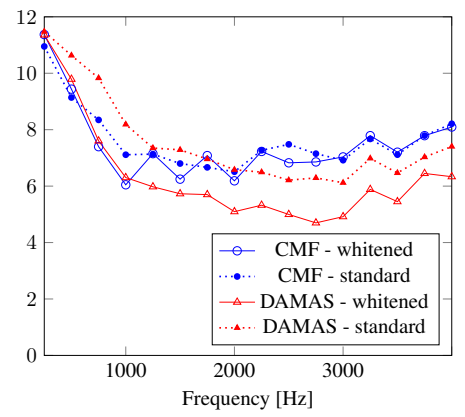

(b) $n_{\text {samp }}=1000, \rho_{\text {rel }}=0.05$.

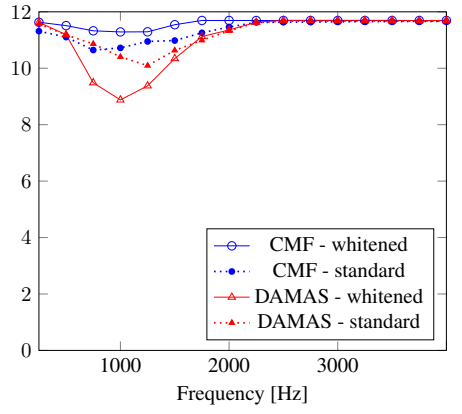

(c) $n_{\text {samp }}=1000, \rho_{\text {rel }}=0.1$.

Figure 7.7.: Error graphs for standard and whitened $C M F / D A M A S$ with $\theta=0$.

\subsubsection{Whitening}

This section examines the weighted imaging methods that were introduced in Chapter 5 . For the (weighted) CMF and DAMAS evaluations we use the same parameters as in Section 7.1.1. The computations are carried out for the frequencies $f \in\{250 \mathrm{~Hz}+j \cdot 250 \mathrm{~Hz}$ : $j=0, \ldots, 15\}$. As weighting (resp. whitening) matrix, the full covariance matrix of the correlation data $\Sigma$ is used and estimated by means of Isserlis' theorem (cf. (4.35)).

Figure 7.7 shows the $L^{2}$ error of each reconstruction method for three different levels of additive noise. We observe that especially for the case with lower additive noise, the whitened method has a considerably lower reconstruction error than its standard weighted counterpart. However, for the scenario with stronger additive noise $\left(\rho_{\text {rel }}=0.1\right)$, the results of whitened and standard methods are very similar for frequencies above $2000 \mathrm{~Hz}$. Below $2000 \mathrm{~Hz}$, the whitened CMF method even produces slightly larger errors.

Figure 7.8 illustrates the effects of whitened methods (Beamforming, CMF and DAMAS) on the imaging result. We observe that the peaks around the source locations are slightly sharper for the weighted imaging methods and the reconstruction error is also reduced. 


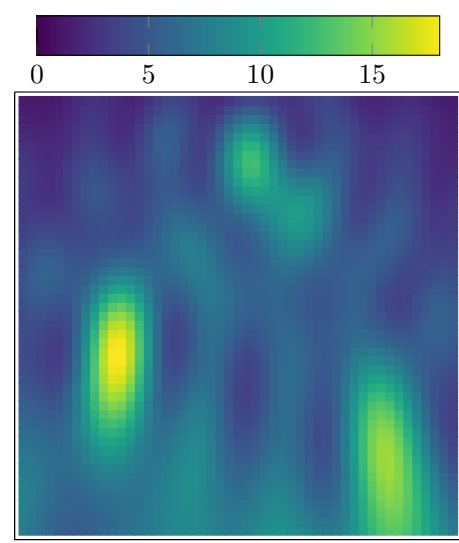

(a) Standard Beamforming
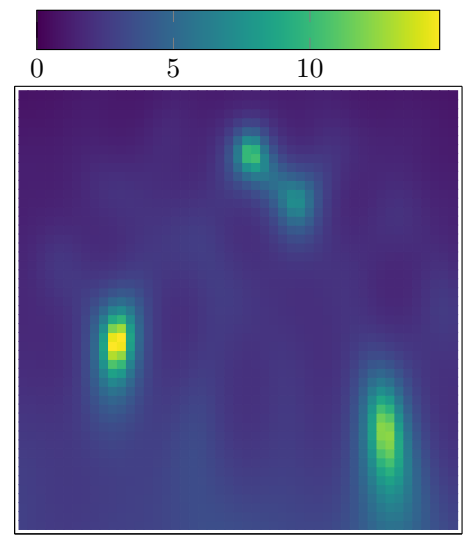

(d) Whitened Beamforming

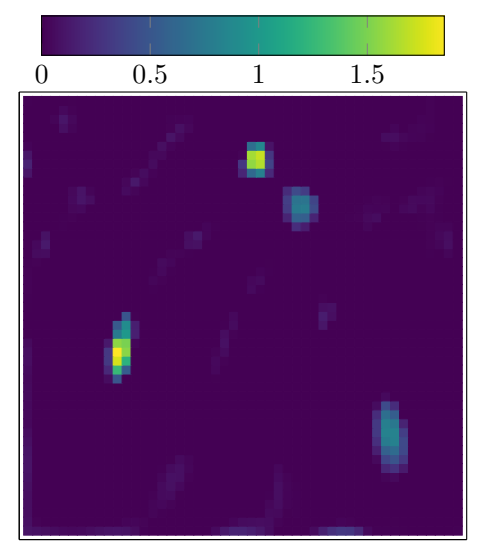

(b) Standard DAMAS, $\mathcal{E}_{2}=8.18$
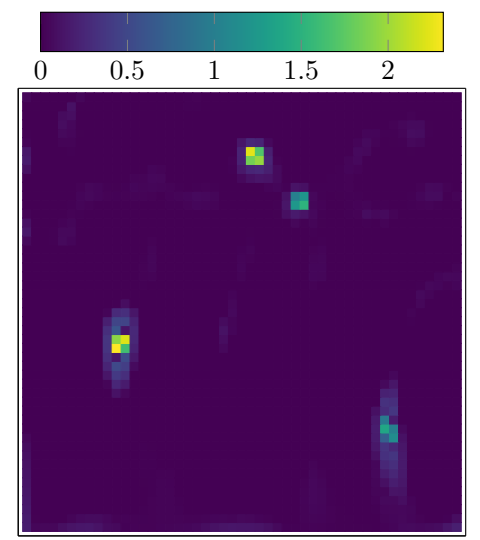

(e) Whitened DAMAS, $\mathcal{E}_{2}=6.30$

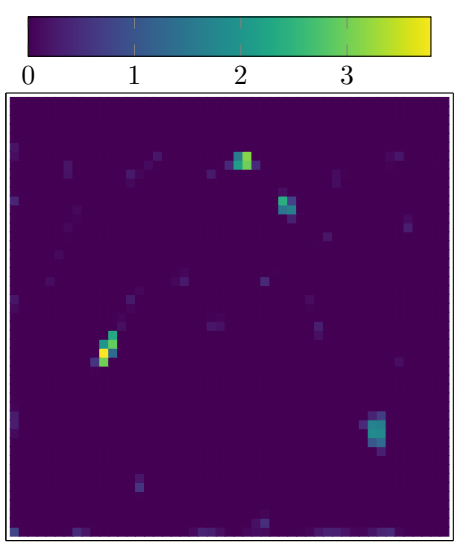

(c) Standard $C M F$, $\mathcal{E}_{2}=7.11$

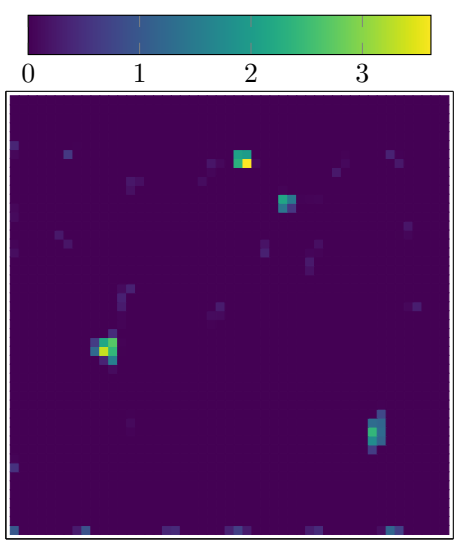

(f) Whitened $C M F$, $\mathcal{E}_{2}=6.05$

Figure 7.8.: Imaging results for $f=1000 \mathrm{~Hz}, n_{\mathrm{samp}}=1000, \rho_{\mathrm{rel}}=0.05, \theta=0$. Full CSM used.

\subsubsection{Spatially extended sources}

So far we considered only a sparse source power distribution with a few approximate point sources. For the next experiment we consider a different source power function that consists of two approximate point sources, an approximate line source and a spatially extended source (see Figure 7.9p). A more complex source configuration is better suited to compare the capability of different reconstruction methods. We consider Conventional Beamforming, the Factorization method (Capon's method) as well as regularized DAMAS and CMF with 10000 FISTA iterations. For the latter two methods we evaluate the standard weighted methods and the whitened version (cf. Section 7.1.3 with the optimal regularization parameter selected from the set

$$
\alpha \in\left\{\alpha_{\text {rel }} \cdot\left\|\underline{y}^{\text {obs }}\right\|_{\underline{Y}}^{2}: \alpha_{\text {rel }}=10^{-15+j}, j=0, \ldots, 15\right\} .
$$




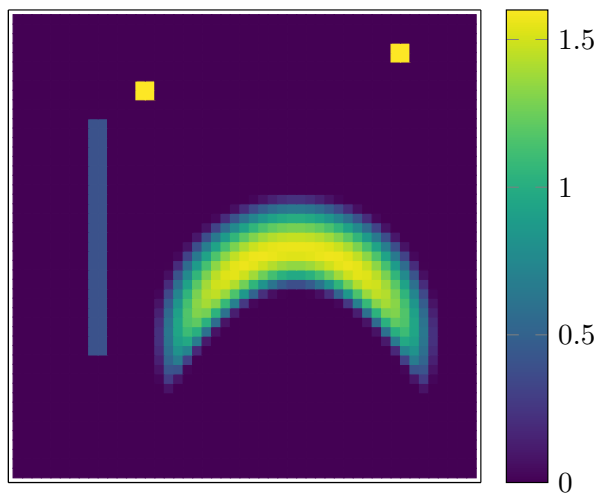

Figure 7.9.: Exact source power function on the reconstruction grid.

See Table 6.2 and Section 6.2 for the definition of the discrete data norms $\|\cdot\|_{\underline{Y}}$.

Figure 7.10 shows the reconstruction results of each method. The results clearly show that neither CBF nor the Factorization method are quantitative reconstruction methods since they miss the correct order of magnitude significantly. Additionally both methods only detect the rough shape of the spatially extended source component.

The two variational methods behave similarly here whereby CMF provides slightly better results than DAMAS. The whitened versions of regularized CMF and DAMAS show each minor improvements i.e. slightly smaller reconstruction errors. Moreover, the whitened CMF reconstruction is the only one that recovers the correct magnitudes of the point sources. We would like to note here that the discrete degree of ill-posedness (i.e. the condition number of the forward matrix) of DAMAS is much larger than that of CMF. The discrete forward operators (cf. (6.9) and (6.10) ) are related by

$$
\underline{T}^{\mathrm{DAMAS}}=D \underline{T}^{\mathrm{CMF} *} \underline{T}^{\mathrm{CMF}},
$$

with a diagonal matrix $D$. Neglecting the influence of $D$, the magnitude of the condition number for the discrete DAMAS forward operator will be squared i.e.

$$
\operatorname{cond}_{\|\cdot\|_{2}}\left(\underline{T}^{\text {DAMAS }}\right) \approx \operatorname{cond}_{\|\cdot\|_{2}}\left(\underline{T}^{\mathrm{CMF}}\right)^{2} \text {. }
$$

Here $\operatorname{cond}_{\|\cdot\|_{2}}(A)=\frac{\sigma_{\max }(A)}{\sigma_{\min }(A)}$ and $\sigma_{\max }(A), \sigma_{\max }(A)$ denote the largest resp. smallest nonzero singular values of a matrix A. However, in this particular example this does not seem to have a strong effect on the reconstruction results. 


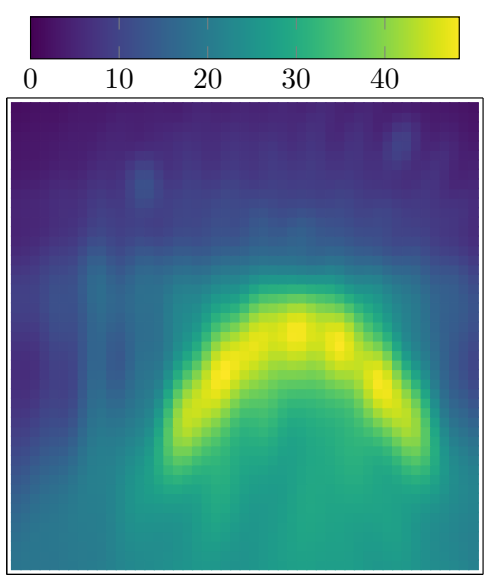

(a) Conventional Beamforming.

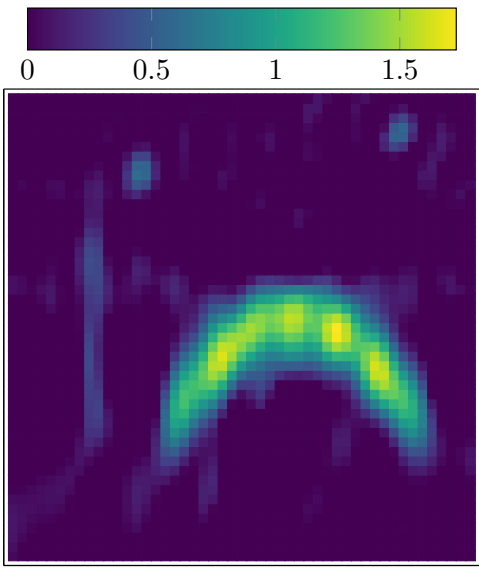

(c) Standard DAMAS. $\mathcal{E}_{2}=4.97, \alpha_{\mathrm{rel}}=10^{-5}$.
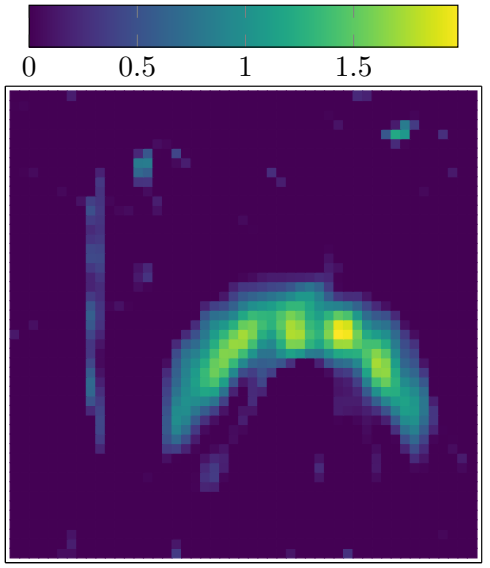

(e) Whitened DAMAS. $\mathcal{E}_{2}=4.73, \alpha_{\mathrm{rel}}=10^{-6}$

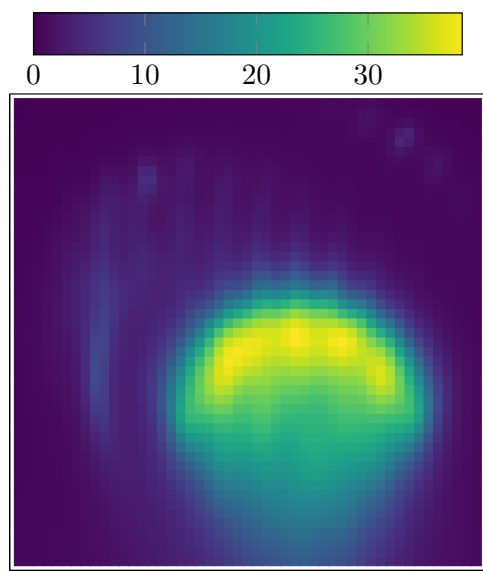

(b) Factorization method.
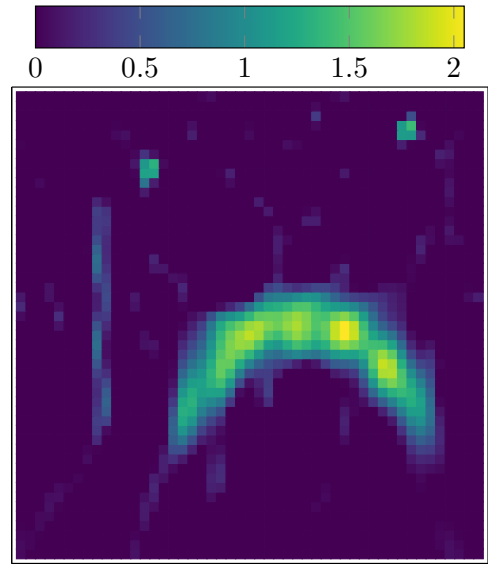

(d) Standard CMF. $\mathcal{E}_{2}=4.50, \alpha_{\mathrm{rel}}=10^{-5}$.
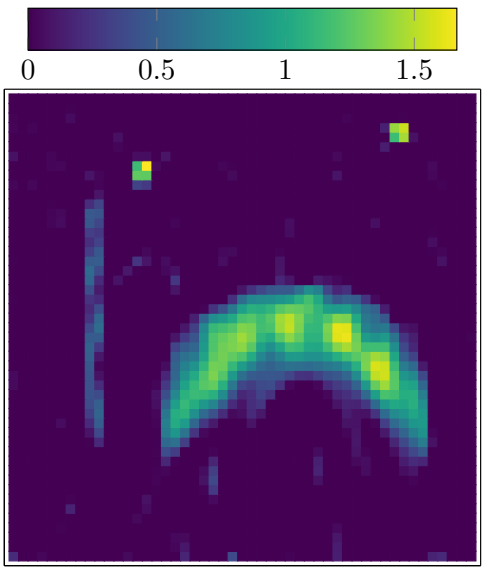

(f) Whitened $C M F$.

$\mathcal{E}_{2}=4.20, \alpha_{\mathrm{rel}}=10^{-11}$.

Figure 7.10.: Comparison of different imaging methods for a spatially extended source. Parameters are $f=2000 \mathrm{~Hz}, n_{\mathrm{samp}}=1000$ and $\rho_{\mathrm{rel}}=0.005$. 


\subsubsection{The Lepskii principle}

With this section we would like to move towards an application oriented evaluation procedure. To this end, we examine regularized CMF reconstructions for $\theta \in\{0,0.5,1\}$ i.e. purely $L^{2}$ resp. $L^{1}$ regularization and an intermediate choice. Since in experimental datasets, we have usually strong boundary layer noise on the CSM diagonal, the evaluations are performed by using only the off-diagonal part of the CSM. We consider regularization parameters

$$
\alpha \in\left\{\alpha_{\text {rel }} \cdot\left\|\underline{y}^{\operatorname{cor}}\right\|_{2}^{2} \alpha_{\text {rel }}=10^{-7+\frac{j-1}{3}}: j=1, \cdots, 25\right\},
$$

where $y^{\text {cor }}$ denotes the vector of off-diagonal correlations of the datapoint (cf. Table 6.2). Moreover, we do not only consider the parameter with the lowest reconstruction error but also employ the Lepskiı principle. As mentioned in the last chapter, this parameter choice rule requires an upper bound $\Psi^{2}$ on the mean square propagated data noise error i.e.

$$
\mathbb{E}\left(\left\|\underline{q}_{\alpha_{j}}-\underline{\hat{q}}_{\alpha_{j}}\right\|_{2}^{2}\right) \leq \Psi^{2}(j) .
$$

Given the data noise covariance matrix $\Sigma$ this upper bound can be estimated by a Monte Carlo simulation, which is explained in more detail below.

Data sampling procedure: Each exact data sample within the Monte Carlo simulation is based on a source power sampling procedure similar to the approach presented in HS17. Similar to the exact source power function examined so far, each sample is given as the sum of scaled indicator functions of squares $R_{s}:=\left\{\mathbf{y}:\left|\mathbf{y}-\mathbf{y}_{s}\right|_{\infty} \leq 0.02\right\}$.

$$
q(\mathbf{y})=\sum_{s=1}^{n_{\text {source }}} \mu_{s} \frac{1}{\left|R_{s}\right|} 1_{R_{s}}(\mathbf{y}) .
$$

The parameters $n_{\text {source }}, \mathbf{y}_{s}, \mu_{s}$ are randomly sampled as follows

- $n_{\text {source }} \sim 1+\operatorname{Pois}(3)$, where Pois(3) denotes the Poisson distribution with parameter 3.

- $\mathbf{y}_{s}$ is sampled from a discrete uniform distribution on a equidistant grid on $\Omega$ with resolution $\Delta_{x y}=0.005 \mathrm{~m}$.

- $\mu_{s}$ is sampled from a Rayleigh distribution Ray(10) with parameter 10.

Monte Carlo algorithm: Based on the described source sampling we can estimate the expected mean square error $\Psi$ by a Monte Carlo estimate $\Psi^{\mathrm{sim}}$. The procedure for the Monte Carlo simulation is summarized in Algorithm 2. 


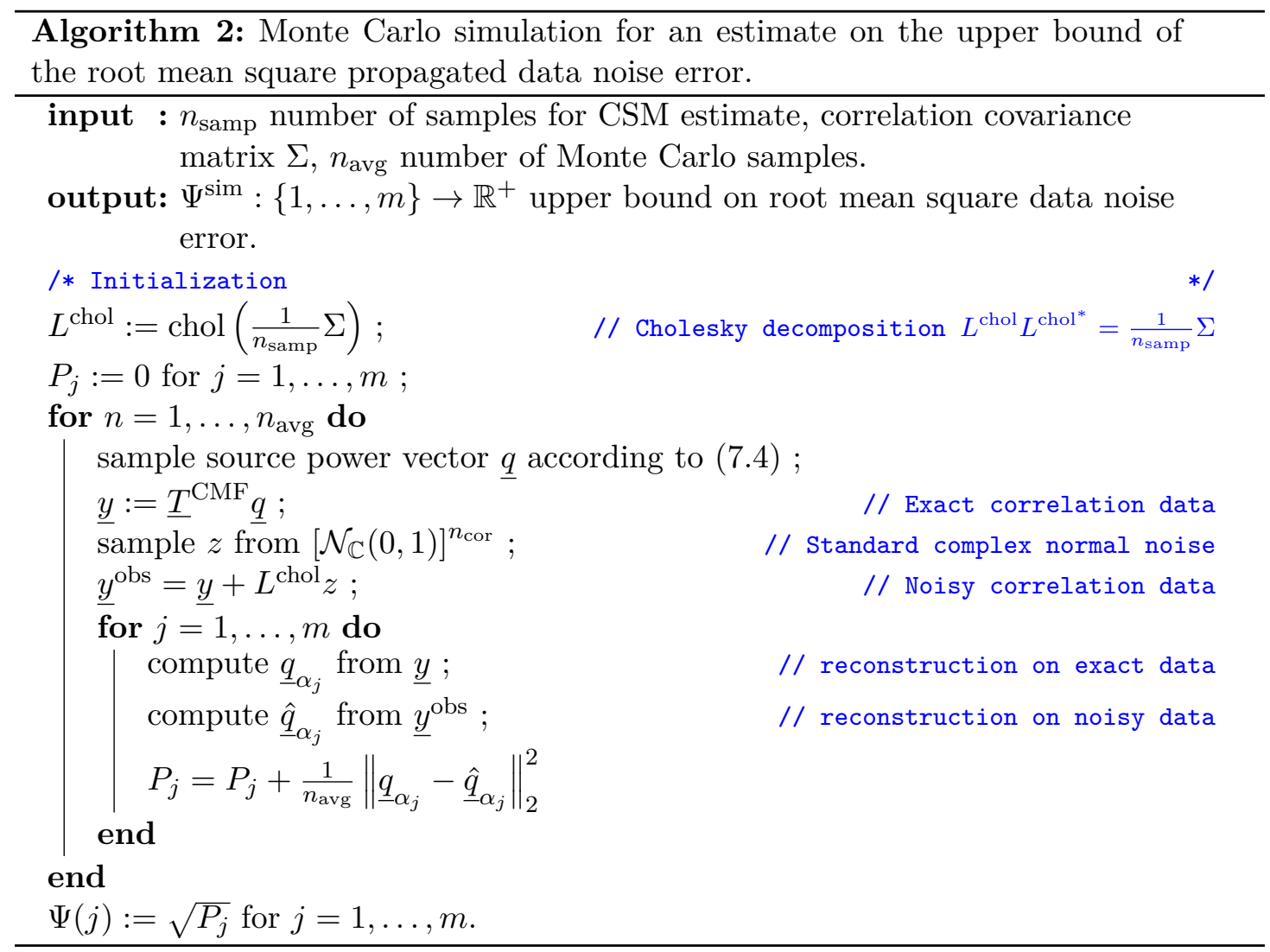

Model for the (propagated) data noise error: We briefly discuss the effects that contribute to the data noise and some alternative approaches to bound the propagated data noise error. Note that the the data noise error

$$
\left\|\underline{C}^{\mathrm{obs}}-\underline{C}\right\|_{F}^{2}
$$

is driven by two causes:

1. the additive measurement noise $\epsilon$ (7.1),

2. the finite number of signal samples $n_{\text {samp }}$.

In our synthetic model (see Section 7.1), the first one is driven by the matrix

$$
Z^{\epsilon}:=\frac{1}{n_{\mathrm{samp}}} \sum_{j=1}^{n_{\mathrm{samp}}} \epsilon^{(j)} \epsilon^{(j) *}
$$


and independent of the true source power function. The second one is driven by the matrix

$$
Z^{q}:=\left[\frac{1}{n_{\mathrm{samp}}} \sum_{j=1}^{n_{\mathrm{samp}}}\left[\underline{\mathcal{G}}\left(\eta^{(j)} \odot \sqrt{\underline{q}}\right)\right]\left[\underline{\mathcal{G}}\left(\eta^{(j)} \odot \sqrt{\underline{q}}\right)\right]^{*}\right]-\underline{C}
$$

and depends on the true source power function. For a correlation value of two distinct microphones, both quantities are expected to decay with the rate $\mathcal{O}\left(\frac{1}{n_{\text {samp }}}\right)$. The relation between the two error sources will be illustrated by a small computational example. We examine the error on the lower triangular part of the cross spectral matrix without the diagonal i.e. for 25 microphones the number of considered correlations is given by

$$
n_{\mathrm{corr}}=\frac{\left(n_{\mathrm{mic}}-1\right) n_{\mathrm{mic}}}{2}=300 .
$$

We set $n_{\text {samp }}=1000$ and consider the two error measures

$$
\begin{aligned}
\mathcal{E}_{\text {meas }} & :=\sqrt{\mathbb{E}\left(\sum_{m>l}\left|Z_{m l}^{\epsilon}\right|^{2}\right)}=\rho_{\text {abs }}^{2} \sqrt{\frac{n_{\text {corr }}}{n_{\text {samp }}}} \\
\mathcal{E}_{\text {source }} & :=\sqrt{\mathbb{E}\left(\sum_{m>l}\left|Z_{m l}^{q}\right|^{2}\right)} .
\end{aligned}
$$

If we consider normalized sources with

$$
\sqrt{\sum_{n=1}^{N}\left|q_{n}\right|}=1
$$

the first error 7.5 is given explicitly, whereas the value of 7.6$)$ depends on $q$. To get an estimate of the order of magnitude of $\mathcal{E}_{\text {source }}$, we sample $n_{\text {source }}=100$ source power functions by the procedure described above. Moreover, the sampled vectors are normalized according to (7.7) and hence $\rho_{\text {rel }}=\rho_{\text {abs. }}$. Figure 7.11 shows both error measures plotted over the additive noise magnitude $\rho_{\text {rel }}$. In practice the relation between the two errors will depend on the specific properties of the measurement setup (e.g. test section, microphone types or frequency range).

Remark 7.1 (Estimation of the propagated data noise error).

In this chapter we do not impose any assumptions on the dominance of a specific error component (i.e. $\mathcal{E}_{\text {source }}$ or $\mathcal{E}_{\text {meas }}$ ). Therefore, the approximation procedure for the mean square propagated data noise error (Algorithm 2) was chosen such that a dependency on the source power function is allowed. If the data is measured in a regime where $\mathcal{E}_{\text {meas }}$ dominates one may argue that the propagated data noise error is also controlled by an upper bound that is independent of the source power function q. In such cases we can drop the source sampling procedure in Algorithm 2. Moreover, for classical Tikhonov reg- 
ularization (i.e. only $L^{2}$ penalization), the tools of regularization theory with variational inequalities (see e.g. WH12, Theorem 3.3]) imply an error splitting of the form

$$
\left\|\underline{\hat{q}}_{\alpha}-\underline{q}\right\|_{2}^{2} \leq 2 \frac{\left\|\underline{C}^{\mathrm{obs}}-\underline{C}\right\|_{F}^{2}}{\alpha}+\Phi(\alpha),
$$

with an increasing function $\Phi$. Here the Lepskiน principle can be applied in a similar manner, where the first summand plays the role of the propagated data noise error. In that case we get explicitly

$$
\mathbb{E}\left(2 \frac{\left\|\underline{C}^{\text {obs }}-\underline{C}\right\|_{F}^{2}}{\alpha}\right)=\frac{2}{\alpha} \operatorname{tr}(\Sigma)
$$

with the trace of the correlation covariance matrix $\operatorname{tr}(\Sigma)=\sum_{n=1}^{n_{\text {mic }}^{2}} \Sigma_{n n}$.

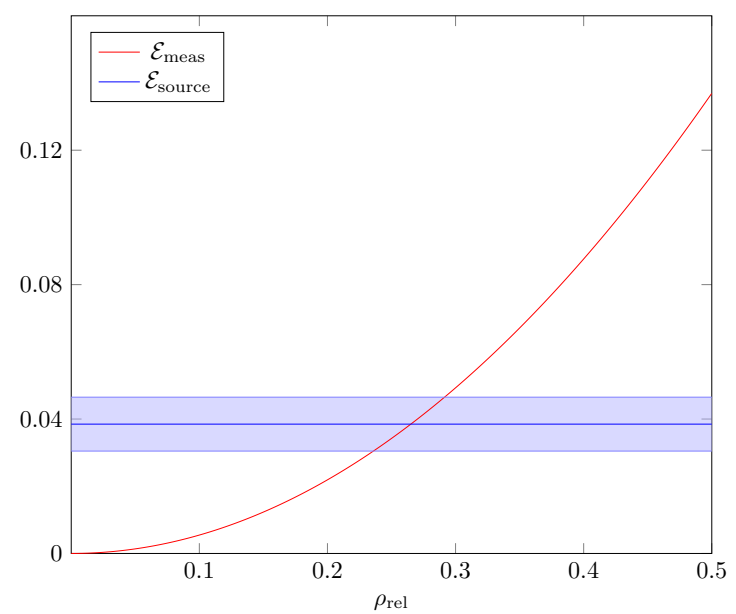

Figure 7.11.: Illustration of the data error measures (7.5) and 7.6) for the computational example described in Section 7.1.5 with $f=4000 \mathrm{~Hz}$. The blue stripe around the mean value of $\mathcal{E}_{\text {source }}$ indicates the standard deviation of the set of estimates.

Imaging results: The imaging results for all three penalty functionals are summarized in Figure 7.12. The first column shows the reconstruction result for the optimal index $j^{*}$ with minimum error. The second column shows the reconstruction result for the index $j^{\text {Lep }}$ according to the Lepskil principle. The last column shows the $L^{2}$ reconstruction error and the Monte Carlo estimate $\Psi^{\text {sim }}$. We observe, that the reconstruction errors decrease with increasing $\theta$, for both parameter choices $j^{*}$ and $j^{\text {Lep }}$ respectively. Especially for the Lepskiu parameter choice, the results for the sparsity promoting penalties $\theta \in\{0.5,1\}$ are quite similar whereas the purely $L^{2}$ regularization produces much larger 


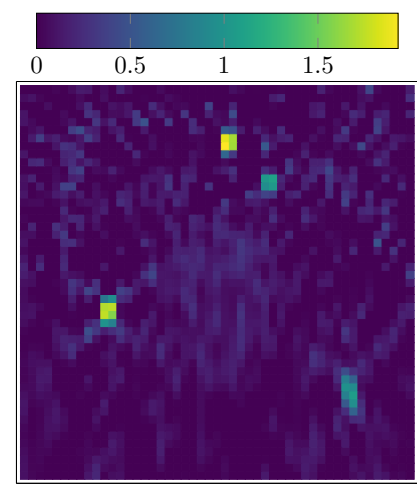

(a) $\alpha=\alpha_{j^{*}}, \theta=0$.

$$
\mathcal{E}_{2}=8.51
$$

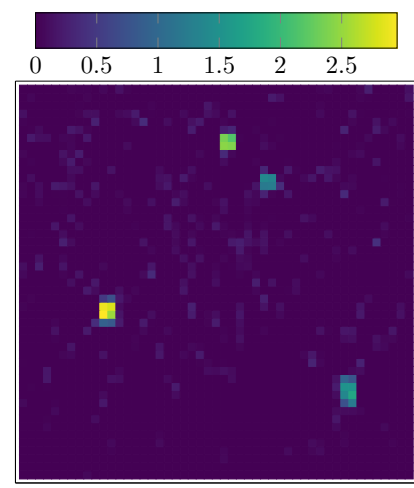

(d) $\alpha=\alpha_{j^{*}}, \theta=0.5$.

$$
\mathcal{E}_{2}=5.04
$$
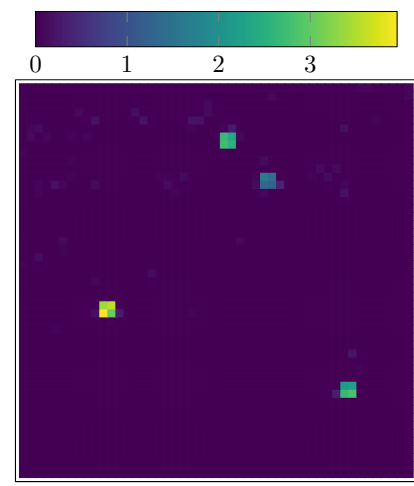

(g) $\alpha=\alpha_{j^{*}}, \theta=1$.

$$
\mathcal{E}_{2}=1.93
$$

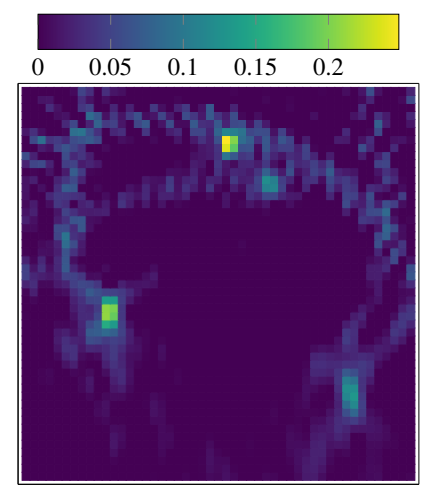

(b) $\alpha=\alpha_{j \mathrm{Lep}}, \theta=0$.

$\mathcal{E}_{2}=11.09$

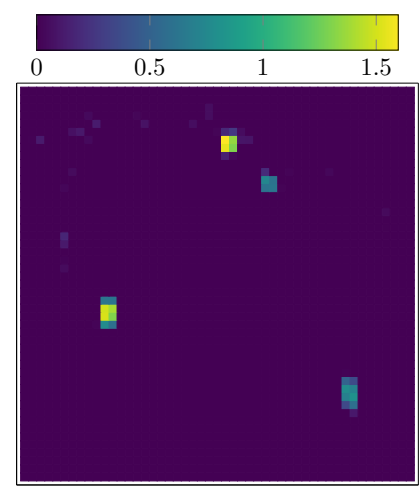

(e) $\alpha=\alpha_{j \mathrm{Lep}}, \theta=0.5$.

$\mathcal{E}_{2}=7.59$

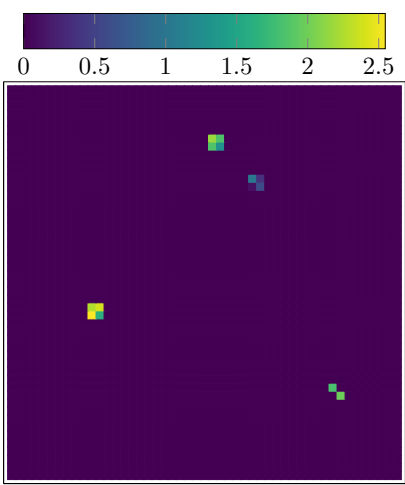

(h) $\alpha=\alpha_{j \text { Lep }}, \theta=1$. $\mathcal{E}_{2}=6.38$

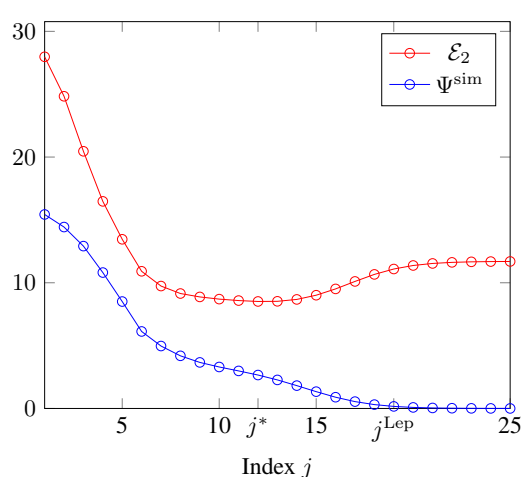

(c) Reconstruction error and simulated data noise error

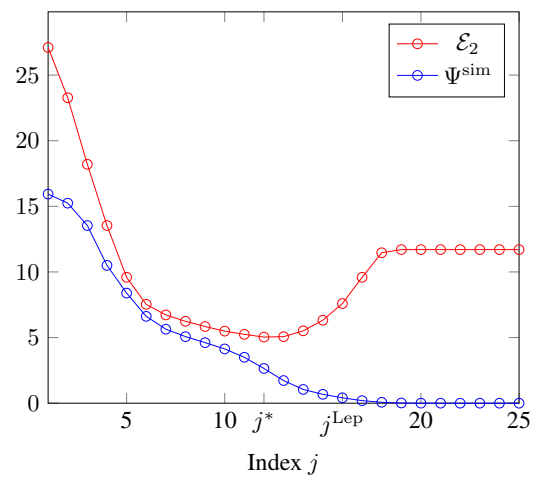

(f) Reconstruction error and simulated data noise error

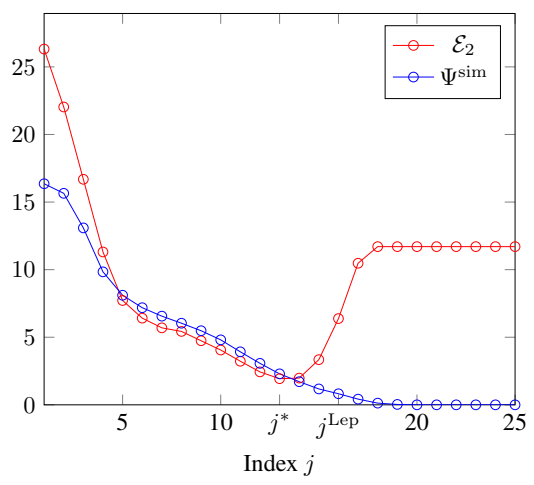

(i) Reconstruction error and simulated data noise error

Figure 7.12.: Imaging results for $f=4000 \mathrm{~Hz}, n_{\mathrm{samp}}=10000, \rho_{\mathrm{rel}}=0.075$. Reconstruction without diagonal entries of the CSM. 
reconstruction errors. This is of course an expected behavior since we considered a very sparse source power function.

\subsection{Experimental data}

To conclude this chapter, we apply the procedure from Section 7.1 .5 to an experimental dataset. The measurement considered here was conducted at the cryogenic wind tunnel in Cologne (DNW-KKK) Ahl13 and examines a scaled half-model of a Dornier 728 aircraft. The measurement parameters that are varied are: Mach number, temperature and the angle of attack i.e. the angle between the cross section plane of the wing and the $x_{1} x_{3}$-plane. This measurement data is widely used as an experimental benchmark dataset (see e.g. [ML18, $\mathrm{BL}^{20}, \mathrm{BHE}^{+} 17$ ]) and is available at the website of the University of Cottbus under the label 'DLR1' [Ben17].

\subsubsection{Aspects of the evaluation procedure}

In experimental practice, there are several principles that are standardly employed in the evaluation process. Therefore we will briefly discuss those principles and point out how this affects the numerical source reconstruction and the representation of the results.

Frequency bands: To reduce noise effects, one often averages source power estimators over a certain frequency band. I.e. for a frequency band $B$ one considers the sum

$$
\sum_{f \in B} \underline{\hat{q}}_{\alpha}(f) .
$$

A typical choice, that is also considered here is the third octave band (cf. ISO 266:1997 [nt97]) with center frequency $f_{0}$ defined by

$$
B\left(f_{0}\right)=\left[2^{-1 / 6} f_{0}, 2^{1 / 6} f_{0}\right] .
$$

Sound pressure level: The reconstructed source power estimator $\underline{\hat{q}}_{\alpha}$ has the unit (Pascal $[\mathrm{Pa}])^{2}$. Instead of the squared sound pressure, one usually considers the sound pressure level (SPL)

$$
\operatorname{SPL}(x)=10 \cdot \log _{10}\left(\frac{x}{p_{0}^{2}}\right)
$$

where $p_{0}$ denotes a reference pressure and is chosen as $p_{0}=2 \cdot 10^{-5} \mathrm{~Pa}$ here.

Parameter choice rule: As in the previous section we employ the Lepskil principle. The Monte Carlo routine for the estimation of the root mean squared data noise error is performed similarly as in the synthetic data case. The sources are sampled as point sources on a grid with resolution $\Delta_{x y}=0.001 \mathrm{~m}$ with $n_{\text {source }} \sim 1+\operatorname{Pois}(4)$ and $\mu_{s} \sim \operatorname{Ray}(0.2)$. The quantity $\Psi^{\mathrm{sim}}$ was only calculated for three frequencies $f \in$ $\{4000 \mathrm{~Hz}, 6000 \mathrm{~Hz}, 8000 \mathrm{~Hz}\}$. For other frequencies, the values of $\Psi^{\text {sim }}$ were interpolated 
by the nearest neighbor principle. The regularization parameters were scaled according to the correlation data i.e.

$$
\alpha=\alpha_{\text {rel }} \cdot\left\|\underline{y}^{\text {cor }}\right\|_{2}^{2}
$$

where $\alpha_{\text {rel }} \in\left\{10^{-7+\frac{j-1}{3}}: j=1, \cdots, 25\right\}$. The FISTA computation was stopped after $n_{\text {iter }}=1000$ iterations.

\subsubsection{Reconstruction results}

We consider regularized CMF evaluations for $\theta \in\{0,0.5,1\}$ with diagonal removal on a planar source region that is aligned to the cross section of the aircraft wing. The array consists of 134 microphones arranged in spiral arms (cf. Figure 7.13). The computations were performed on a grid with resoulution $\Delta_{x y}=0.02 \mathrm{~m}$.

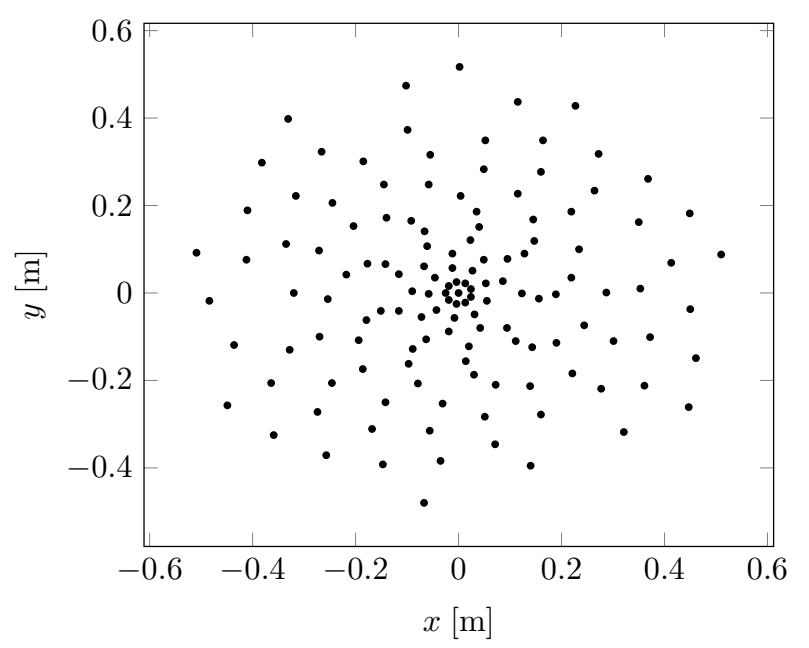

Figure 7.13.: Microphone positions of the experimental dataset.

Figure 7.14 shows third octave band SPL source maps for the center frequencies $4000 \mathrm{~Hz}, 6000 \mathrm{~Hz}$ and $8000 \mathrm{~Hz}$. We observe that the purely $L^{2}$ regularization $(\theta=0)$ shows by far the strongest noise effects. Within the dynamic range of $20 \mathrm{~dB}$, the two sparsity promoting methods reconstruct almost exclusively sources on or close to the aircraft model. However, in contrast to the purely $L^{2}$ regularization $(\theta=0)$ they also tend to suppress weaker source components. 


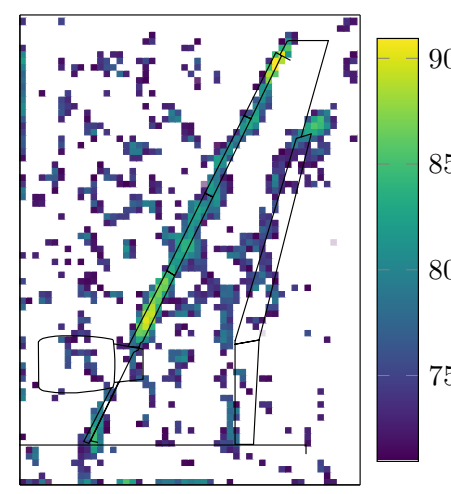

(a) $f_{1 / 3 O c t}=4000 \mathrm{~Hz}$, $\theta=0, \alpha_{\mathrm{rel}}=0.0215$

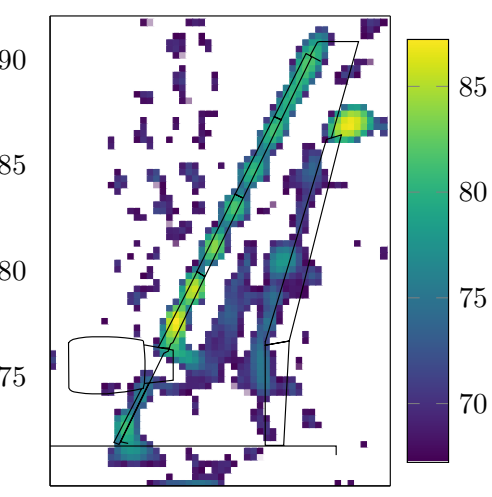

(b) $f_{1 / 3 O c t}=6000 \mathrm{~Hz}$, $\theta=0, \alpha_{\mathrm{rel}}=1$

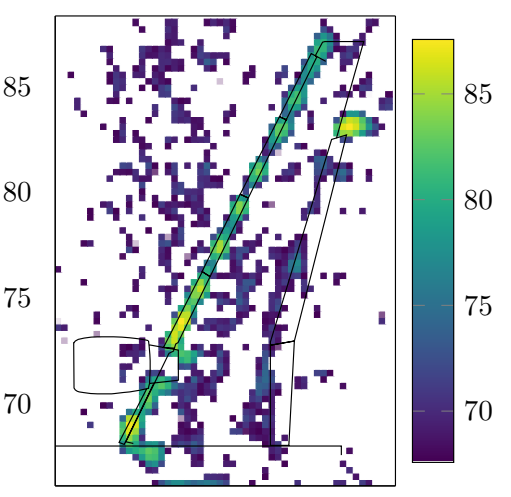

(c) $f_{1 / 30 c t}=8000 \mathrm{~Hz}$, $\theta=0, \alpha_{\text {rel }}=1$

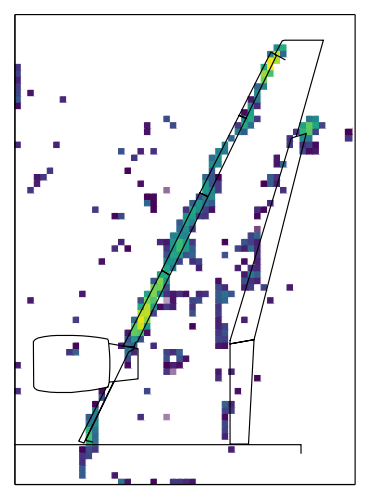

(d) $f_{1 / 3 O c t}=4000 \mathrm{~Hz}$, $\theta=0.5, \alpha_{\mathrm{rel}}=0.0464$

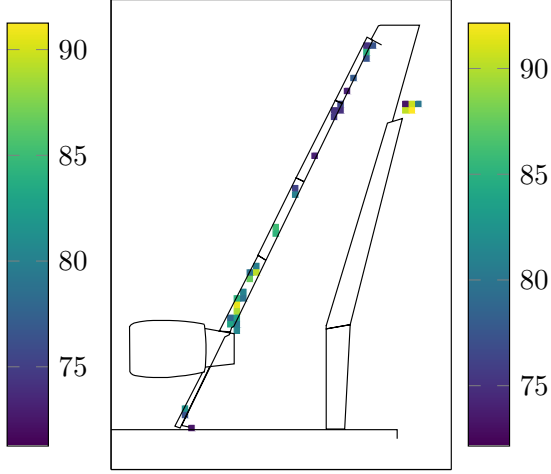

(e) $f_{1 / 3 O c t}=6000 \mathrm{~Hz}$, $\theta=0.5, \alpha_{\mathrm{rel}}=0.2154$

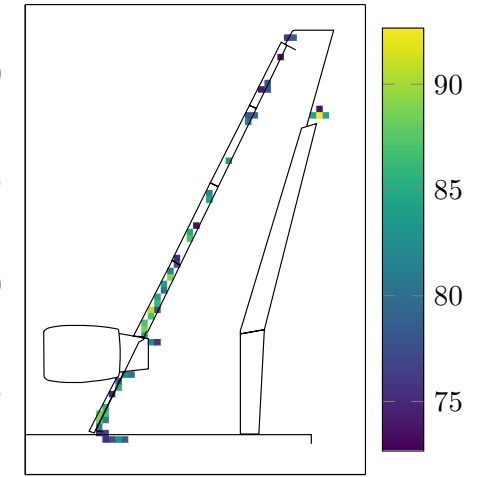

(f) $f_{1 / 3 O c t}=8000 \mathrm{~Hz}$, $\theta=0.5, \alpha_{\mathrm{rel}}=0.1$

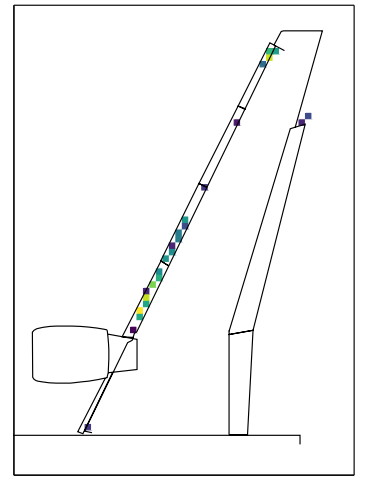

(g) $f_{1 / 3 O c t}=4000 \mathrm{~Hz}$,

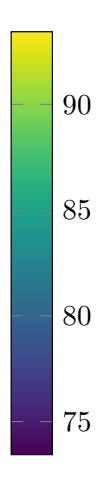

75

$$
\theta=1, \alpha_{\mathrm{rel}}=0.1
$$

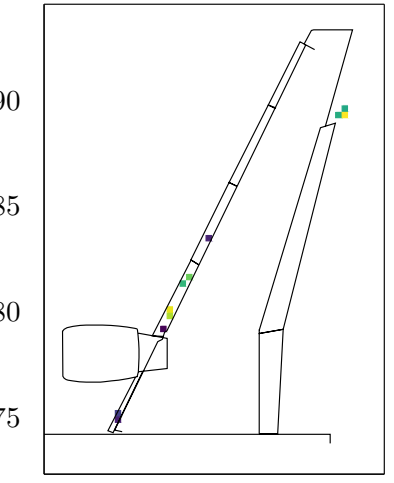

(h) $f_{1 / 3 O c t}=6000 \mathrm{~Hz}$,

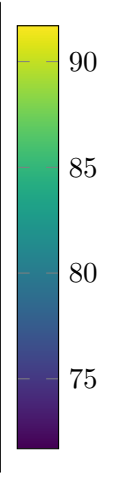

$\theta=1, \alpha_{\mathrm{rel}}=0.1$

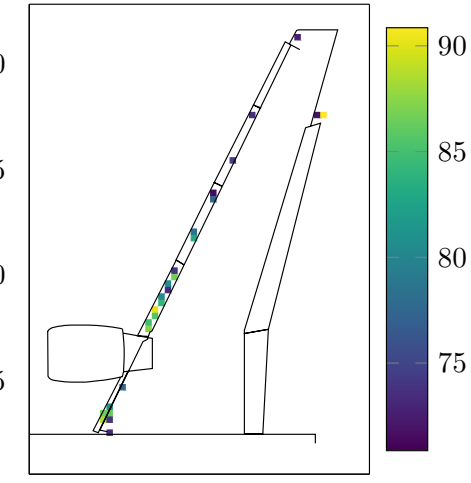

(i) $f_{1 / 3 O c t}=8000 \mathrm{~Hz}$, $\theta=1, \alpha_{\text {rel }}=0.0464$

Figure 7.14.: Imaging results for the experimental benchmark dataset using regularized CMF with $\alpha \propto \alpha_{\text {rel }}(7.8)$ chosen by the Lepskiı principle.

Reconstruction without diagonal entries of the CSM. Dynamic range $20 \mathrm{~dB}$, Mach number: 0.15, angle of attack: $3^{\circ}$. 


\section{Chapter}

\section{Conclusions and Outlook}

This work presented a mathematical formulation and analysis of the inverse source problem in aeroacoustics. We covered the fields of sound propagation modelling (Chapter 2), mathematical analysis of the infinite dimensional (Chapter 3 respectively finite dimensional problem (Chapter 4 and 5) and numerical methods (Chapter 6 and 7). In each of these fields, natural and interesting questions emerge, either aimed at an extension of the theoretical framework or an adaption to experimental conditions.

\section{Stability estimates and variational source conditions}

A natural extension of the results presented in Chapter 2 would be the proof of a conditional stability estimate. For the inverse source problem discussed in this work such an estimate would be of the form

$$
l\left(q_{1}, q_{2}\right) \leq C \varphi\left(\left\|\mathcal{C}\left(q_{1}\right)-\mathcal{C}\left(q_{2}\right)\right\|_{\mathrm{HS}\left(L^{2}(\mathbb{M})\right)}\right) \text { for } q_{1}, q_{2} \in \mathcal{B} \subset L^{2}(\Omega) .
$$

Here $l$ denotes a loss function, $\varphi:[0, \infty) \rightarrow[0, \infty)$ an index function (i.e. continuous and strictly increasing with $\varphi(0)=0)$ and $\mathcal{B}$ a subset of the source space, usually containing functions with a certain smoothness. An even stronger result would be the verification of a variational source condition (VSC) (cf. eg. HW15]). The derivation of a VSC would provide error bounds and convergence rates of the reconstruction error. As a starting point one may consider the framework presented e.g. in Wei19] and transfer the ideas to the inverse source problem studied in this thesis.

\section{Model order reduction}

Recall the factorization of the discrete forward operator

$$
\underline{\mathcal{G}} \underline{M q}_{\underline{\mathcal{G}^{*}}} \underline{ }^{*}
$$

with $\underline{\mathcal{G}} \in \mathbb{C}^{n_{\text {mic }} \times N}$ and $\underline{q} \in \mathbb{R}^{N}$. For standard implementations, one evaluation of the forward operator has the complexity $\mathcal{O}\left(n_{\text {mic }}^{2} N\right)$. Thus for large arrays and fine dis- 
cretizations, it is desirable to reduce the complexity of the discrete problem to ensure numerical feasibility. The forward operator studied within this work fits exactly into the class of problems that is examined in the recent work by Dölz, Egger \& Schlottbom [DES20. Hence, the tools presented therein may also be useful for the aeroacoustic source power reconstruction problem.

In recent years, the use of MEMS (Micro-Electro-Mechanical Systems) microphones in aeroacoustic measurement has become increasingly popular (see e.g. [EGK $\left.{ }^{+} 20\right]$ ). A huge advantage of MEMS microphones is their extremely low price compared to classic measurement microphones. Therefore, large arrays using 10 or even 100 times more microphones than standard arrays can be realized. Having in mind that the numerical complexity scales with $n_{\text {mic }}^{2}$, model order reduction methods will become more and more important for the evaluation of aeroacoustic measurements.

\section{Sound propagation with numerical methods}

The assumptions on the sound propagation model used in this thesis are strongly simplifying compared to realistic measurement conditions. Numerical schemes for the solution of the (convected) Helmholtz equation such as the Boundary Element Method (BEM) or the Finite Element Method (FEM) could be employed to get more realistic sound propagation models (i.e. volume potential operators). A straightforward generic procedure for such an approach is based on the following steps

1. Fix a set of basis functions $X_{N}:=\left\{\phi_{n}\right\}_{n=1}^{N} \subset L^{2}(\Omega)$.

2. For each $\phi_{n} \in X_{N}$ solve a numerical BEM or FEM scheme (that may incorporate complex geometries and boundary conditions) to compute the pressure field $p_{\phi_{n}}\left(\mathbf{x}_{m}\right)$ at the microphone positions $\left\{\mathbf{x}_{m}\right\}_{m=1}^{n_{\text {mic }}}$ generated by $\phi_{n}$.

3. Set up the the volume potential operator by

$$
\underline{\mathcal{G}}_{m n}=p_{\phi_{n}}\left(\mathbf{x}_{m}\right) \text {. }
$$

Moreover, one may ask if our source power reconstruction problem allows for a formulation that avoids the explicit evaluation of the forward operator (known as all-at-once formulations [Kal16, Kal18]). For the deterministic inverse source problem, such an approach has been investigated in [KKG18, GKK18, HK21]. Applying those ideas for the random inverse source problem with correlation data we obtain formally the following system of equations: find $q$ and $P$ such that

$$
\begin{array}{ll}
F(q, P)=0 & \text { (physical model) } \\
\operatorname{Cov}(P)=C & \text { (observation map). }
\end{array}
$$

Where $P$ denotes the random pressure field and the physical model $(8.1 \mathrm{a})$ is given by a stochastic partial differential equation (SPDE) rather than a partial differential equation (PDE) as in the deterministic case. Therefore, a numerical realization of this approach is not straightforward, since we need a computational scheme that efficiently implements 8.1a. 


\section{Appendix}

\section{Auxiliary Statements}

Proof of Proposition 2.3 [Integration by parts formula]

Let $U \subset \mathbb{R}^{d}(d \geq 2)$ be a bounded domain with Lipschitz boundary and $u, v \in C^{2}(\bar{U})$. Then the following integral equation is valid

$$
\begin{aligned}
\int_{U} u(\mathbf{y})\left(L_{k} v\right)(\mathbf{y}) d \mathbf{y}= & \int_{U}\left(L_{k}^{\top} u\right)(\mathbf{y}) v(\mathbf{y}) d \mathbf{y} \\
& +\oint_{\partial U}\{u(\mathbf{y}) \nabla v(\mathbf{y})-v(\mathbf{y}) \nabla u(\mathbf{y})\} \cdot \mathbf{n} d s(\mathbf{y}) \\
& +|\mathbf{m}|^{2} \oint_{\partial U}\left\{v(\mathbf{y}) \frac{\partial u}{\partial y_{1}} n_{1}-u(\mathbf{y}) \frac{\partial v}{\partial y_{1}} n_{1}\right\} d s(\mathbf{y}) \\
& +2 i k|\mathbf{m}| \oint_{\partial U} u(\mathbf{y}) v(\mathbf{y}) n_{1} d s(\mathbf{y})
\end{aligned}
$$

where $\mathbf{n}=\left(n_{1}, \ldots, n_{d}\right)^{\top} \in \mathbb{R}^{d}$ denotes the unit normal vector of $\partial U$, pointing outwards of $U$.

Proof. The proof relies on Green's second formula (cf. [CK13, p. 17])

$$
\int_{U}(\phi(\mathbf{y}) \Delta \psi(\mathbf{y})-\Delta \phi(\mathbf{y}) \psi(\mathbf{y})) d \mathbf{y}=\oint_{\partial U}(\phi(\mathbf{y}) \nabla \psi(\mathbf{y})-\psi(\mathbf{y}) \nabla \phi(\mathbf{y})) \cdot \mathbf{n} d s(\mathbf{y})
$$

and multidimensional integration by parts (cf. [Gri11, Theorem 1.5.3.1 p.52])

$$
\int_{U} \phi(\mathbf{y}) \frac{\partial}{\partial y_{i}} \psi(\mathbf{y}) d \mathbf{y}=-\int_{U} \frac{\partial}{\partial y_{i}} \phi(\mathbf{y}) \psi(\mathbf{y}) d \mathbf{y}+\oint_{\partial U} \phi(\mathbf{y}) \psi(\mathbf{y}) n_{i} d s(\mathbf{y})
$$

where $\phi, \psi \in C^{2}(\bar{U})$. We have

$$
\int_{U} u(\mathbf{y})\left(L_{k} v\right)(\mathbf{y}) d \mathbf{y}=\int_{U} u(\mathbf{y})\left\{k^{2}+2 i k|\mathbf{m}| \frac{\partial}{\partial y_{1}}-|\mathbf{m}|^{2} \frac{\partial^{2}}{\partial y_{1}^{2}}+\Delta\right\} v(\mathbf{y}) d \mathbf{y} .
$$


By the calculation rules above we obtain

$$
\begin{aligned}
& \int_{U} u(\mathbf{y}) 2 i k|\mathbf{m}| \frac{\partial}{\partial y_{1}} v(\mathbf{y}) d \mathbf{y} \stackrel{\text { A.2 }}{=}-\int_{U} 2 i k|\mathbf{m}| \frac{\partial}{\partial y_{1}} u(\mathbf{y}) v(\mathbf{y}) d \mathbf{y} \\
&+\oint_{\partial U} 2 i k|\mathbf{m}| u(\mathbf{y}) v(\mathbf{y}) n_{1} d s(\mathbf{y}) \\
&|\mathbf{m}|^{2} \int_{U} u(\mathbf{y}) \frac{\partial}{\partial y_{1}^{2}} v(\mathbf{y}) d \mathbf{y} \stackrel{\text { twice } \stackrel{\text { A. } 2)}{=}}{ } \int_{U}|\mathbf{m}|^{2} \frac{\partial^{2}}{\partial y_{1}^{2}} u(\mathbf{y}) v(\mathbf{y}) d \mathbf{y} \\
&+\oint_{\partial U}|\mathbf{m}|^{2}\left\{u(\mathbf{y}) \frac{\partial}{\partial y_{1}} v(\mathbf{y})-\frac{\partial}{\partial y_{1}} u(\mathbf{y}) v(\mathbf{y})\right\} n_{1} d s(\mathbf{y}) \\
& \int_{U} u(\mathbf{y}) \Delta v(\mathbf{y}) d \mathbf{y} \stackrel{\text { A.1 })}{=} \int_{U} \Delta u(\mathbf{y}) v(\mathbf{y}) d \mathbf{y} \\
&+\oint_{\partial U}\{u(\mathbf{y}) \nabla v(\mathbf{y})-v(\mathbf{y}) \nabla u(\mathbf{y})\} \cdot \mathbf{n} d s(\mathbf{y}) .
\end{aligned}
$$

Proof of Theorem 2.14 [Representation theorem - free field]

Let $U \subset \mathbb{R}^{d}$ be a bounded domain of class $C^{2}$ and $u \in C^{2}\left(\mathbb{R}^{d} \backslash \bar{U}\right) \cap C^{1}\left(\mathbb{R}^{d} \backslash U\right)$ such that $L_{k} u=0$ in $\mathbb{R}^{d} \backslash \bar{U}$. Furthermore, assume that $v(\mathbf{y}):=\exp \left(\frac{|\mathbf{m}| i k}{\beta} x_{1}\right) u\left(\mathbf{T}^{-1} \mathbf{x}\right)$ satisfies the Sommerfeld radiation condition (2.15). Then we have the following representation formula for any $\mathbf{x} \in \mathbb{R}^{d} \backslash \bar{U}$

$$
\begin{aligned}
u(\mathbf{x})= & \oint_{\partial U}\left\{u(\mathbf{y}) \nabla_{\mathbf{y}} g(\mathbf{x}, \mathbf{y})-g(\mathbf{x}, \mathbf{y}) \nabla_{\mathbf{y}} u(\mathbf{y})\right\} \cdot \mathbf{n} d s(\mathbf{y}) \\
& +|\mathbf{m}|^{2} \oint_{\partial U}\left\{g(\mathbf{x}, \mathbf{y}) \frac{\partial u}{\partial \mathbf{y}_{1}}(\mathbf{y})-u(\mathbf{y}) \frac{\partial g}{\partial \mathbf{y}_{1}}(\mathbf{x}, \mathbf{y})\right\} n_{1} d s(\mathbf{y}) \\
& -2|\mathbf{m}| i k \oint_{\partial U} u(\mathbf{y}) g(\mathbf{x}, \mathbf{y}) n_{1} d s(\mathbf{y})
\end{aligned}
$$

where $\mathbf{n}=\left(n_{1}, \ldots, n_{d}\right)^{\top}$ is the outer unit normal vector of $\partial U$.

Proof. By Proposition 2.2, the function

$$
v(\mathbf{x})=\exp \left(\frac{|\mathbf{m}| i k}{\beta} x_{1}\right) u\left(\mathbf{T}^{-1} \mathbf{x}\right)
$$

solves the ordinary Helmholtz equation for wavenumber $\frac{k}{\beta}$ on $\mathbb{R}^{d} \backslash \overline{\mathbf{T}(U)}$. We denote by $g_{0}$ the no-flow Green's function for wavenumber $\frac{k}{\beta}$ and employ the representation formula for the ordinary Helmholtz equation (see [CC06. Theorem 3.1 p. 52] for $d=2$ and [CK13, Theorem 2.5 p.19] for $d=3$ ). Moreover we employ the fact that for a vector field $\mathbf{f} \in\left[C^{1}(U)\right]^{d}$ the divergence theorem together with the change of variables $\hat{\mathbf{y}}=\mathbf{T y}$ 
implies

$$
\begin{aligned}
\oint_{\mathbf{T}(U)} \mathbf{f}(\hat{\mathbf{y}}) \cdot \mathbf{n}_{\partial \mathbf{T}(U)} d s(\hat{\mathbf{y}}) & =\int_{\mathbf{T}(U)}(\operatorname{div}(\mathbf{f}))(\hat{\mathbf{y}}) d \hat{\mathbf{y}}=|\operatorname{det} \mathbf{T}| \int_{U}(\operatorname{div}(\mathbf{f}))(\mathbf{T y}) d \mathbf{y} \\
& =|\operatorname{det} \mathbf{T}| \int_{U}(\operatorname{div}(\tilde{\mathbf{f}}))(\mathbf{y}) d \mathbf{y}=|\operatorname{det} \mathbf{T}| \oint_{U} \tilde{\mathbf{f}}(\mathbf{y}) \cdot \mathbf{n}_{\partial U} d s(\mathbf{y})
\end{aligned}
$$

where

$$
\tilde{\mathbf{f}}(\mathbf{y}):=\mathbf{T}^{-1} \mathbf{f}(\mathbf{T y}) \quad \text { such that } \quad(\operatorname{div}(\tilde{\mathbf{f}}))(\mathbf{y})=(\operatorname{div}(\mathbf{f}))(\mathbf{T y}) .
$$

That yields for any $\mathbf{z} \in \mathbb{R}^{d} \backslash \overline{\mathbf{T}(U)}$

$$
\begin{aligned}
v(\mathbf{z})= & \exp \left(\frac{|\mathbf{m}| i k}{\beta} z_{1}\right) u\left(\mathbf{T}^{-1} \mathbf{z}\right) \\
= & \oint_{\partial \mathbf{T}(U)}\left\{v(\mathbf{y}) \nabla_{\mathbf{y}} g_{0}(\mathbf{z}, \mathbf{y})-g_{0}(\mathbf{z}, \mathbf{y}) \nabla_{\mathbf{y}} v(\mathbf{y})\right\} \cdot \mathbf{n}_{\partial \mathbf{T}(U)} d s(\mathbf{y}) \\
= & \frac{1}{\beta} \oint_{\partial U}\left\{v(\mathbf{T y}) \mathbf{T}^{-1}\left(\nabla_{\mathbf{y}} g_{0}\right)(\mathbf{z}, \mathbf{T y})-g_{0}(\mathbf{z}, \mathbf{T y}) \mathbf{T}^{-1}\left(\nabla_{\mathbf{y}} v\right)(\mathbf{T y})\right\} \cdot \mathbf{n}_{\partial U} d s(\mathbf{y}) \\
= & \frac{1}{\beta} \oint_{\partial U}\left\{\exp \left(\frac{|\mathbf{m}| i k}{\beta^{2}} y_{1}\right) u(\mathbf{y}) \mathbf{T}^{-1}\left(\nabla_{\mathbf{y}} g_{0}\right)(\mathbf{z}, \mathbf{T y})\right\} \cdot \mathbf{n}_{\partial U} d s(\mathbf{y}) \\
& -\frac{1}{\beta} \oint_{\partial U}\left\{g_{0}(\mathbf{z}, \mathbf{T y}) \exp \left(\frac{|\mathbf{m}| i k}{\beta^{2}} y_{1}\right) \mathbf{T}^{-1}\left(\frac{|\mathbf{m}| i k}{\beta} u(\mathbf{y})\left(\begin{array}{l}
1 \\
0 \\
0
\end{array}\right)+\mathbf{T}^{-1} \nabla_{\mathbf{y}} u(\mathbf{y})\right)\right\} \cdot \mathbf{n}_{\partial U} d s(\mathbf{y}) .
\end{aligned}
$$

From now on we omit the index of the normal vector, and $\mathbf{n}$ always denotes the outer normal vector of the corresponding integration domain. Note the following identities that will be used further on

$$
\begin{aligned}
g(\mathbf{x}, \mathbf{y})= & \frac{1}{\beta} \exp \left(-\frac{|\mathbf{m}| i k}{\beta^{2}}\left(x_{1}-y_{1}\right)\right) g_{0}(\mathbf{T x}, \mathbf{T y}) \\
\mathbf{T}^{-2} \nabla_{\mathbf{y}} g(\mathbf{x}, \mathbf{y})= & \frac{1}{\beta} \exp \left(\frac{-|\mathbf{m}| i k}{\beta^{2}}\left(x_{1}-y_{1}\right)\right) \mathbf{T}^{-1}\left(\nabla_{\mathbf{y}} g_{0}\right)(\mathbf{T x}, \mathbf{T y}) \\
& +\frac{1}{\beta} \frac{|\mathbf{m}| i k}{\beta^{2}} \exp \left(\frac{-|\mathbf{m}| i k}{\beta^{2}}\left(x_{1}-y_{1}\right)\right) \cdot g_{0}(\mathbf{T x}, \mathbf{T y})\left(\begin{array}{c}
\beta^{2} \\
0 \\
0
\end{array}\right), \\
\mathbf{T}^{-2}= & \mathbf{I}-\left(\begin{array}{rrr}
|\mathbf{m}|^{2} & 0 & 0 \\
0 & 0 & 0 \\
0 & 0 & 0
\end{array}\right) .
\end{aligned}
$$


Setting $\mathbf{z}=$ Tx with $\mathbf{x} \in \mathbb{R}^{d} \backslash \bar{U}$ and multiplying by $\exp \left(\frac{-|\mathbf{m}| i k}{\beta^{2}} x_{1}\right)$ on both sides yields

$$
\begin{aligned}
& u(\mathbf{x})=\frac{1}{\beta} \oint_{\partial U}\left\{\exp \left(\frac{-|\mathbf{m}| i k}{\beta^{2}}\left(x_{1}-y_{1}\right)\right) u(\mathbf{y}) \mathbf{T}^{-1}\left(\nabla_{\mathbf{y}} g_{0}\right)(\mathbf{T x}, \mathbf{T y})\right\} \cdot \mathbf{n} d s(\mathbf{y}) \\
& -\frac{1}{\beta} \oint_{\partial U}\left\{g_{0}(\mathbf{T x}, \mathbf{T y}) \exp \left(\frac{-|\mathbf{m}| i k}{\beta^{2}}\left(x_{1}-y_{1}\right)\right)\right. \\
& \left.\cdot \mathbf{T}^{-1}\left(\frac{|\mathbf{m}| i k}{\beta} u(\mathbf{y})\left(\begin{array}{l}
1 \\
0 \\
0
\end{array}\right)+\mathbf{T}^{-1} \nabla_{\mathbf{y}} u(\mathbf{y})\right)\right\} \cdot \mathbf{n} d s(\mathbf{y})
\end{aligned}
$$

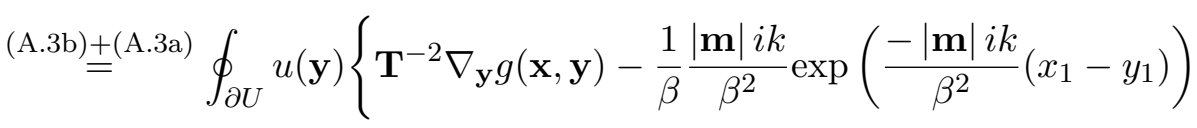

$$
\begin{aligned}
& \left.\cdot g_{0}(\mathbf{T x}, \mathbf{T y})\left(\begin{array}{c}
\beta^{2} \\
0 \\
0
\end{array}\right)\right\} \cdot \mathbf{n} d s(\mathbf{y}) \\
& -\oint_{\partial U} g(\mathbf{x}, \mathbf{y})\left\{\mathbf{T}^{-1}\left(\frac{|\mathbf{m}| i k}{\beta} u(\mathbf{y})\left(\begin{array}{l}
1 \\
0 \\
0
\end{array}\right)+\mathbf{T}^{-1} \nabla_{\mathbf{y}} u(\mathbf{y})\right)\right\} \cdot \mathbf{n} d s(\mathbf{y}) \\
& \stackrel{\text { A.3a) }}{=} \oint_{\partial U} u(\mathbf{y})\left\{\mathbf{T}^{-2} \nabla_{\mathbf{y}} g(\mathbf{x}, \mathbf{y})-\frac{|\mathbf{m}| i k}{\beta^{2}} g(\mathbf{x}, \mathbf{y})\left(\begin{array}{c}
\beta^{2} \\
0 \\
0
\end{array}\right)\right\} \cdot \mathbf{n} d s(\mathbf{y}) \\
& -\oint_{\partial U} g(\mathbf{x}, \mathbf{y})\left\{\mathbf{T}^{-1}\left(\frac{|\mathbf{m}| i k}{\beta} u(\mathbf{y})\left(\begin{array}{l}
1 \\
0 \\
0
\end{array}\right)+\mathbf{T}^{-1} \nabla_{\mathbf{y}} u(\mathbf{y})\right)\right\} \cdot \mathbf{n} d s(\mathbf{y}) \\
& \stackrel{\text { A.3c }}{=} \oint_{\partial U}\left\{u(\mathbf{y}) \nabla_{\mathbf{y}} g(\mathbf{x}, \mathbf{y})\right\} \cdot \mathbf{n} d s(\mathbf{y})-|\mathbf{m}|^{2} \oint_{\partial U} u(\mathbf{y}) \frac{\partial g}{\partial y_{1}}(\mathbf{x}, \mathbf{y}) n_{1} d s(\mathbf{y}) \\
& -|\mathbf{m}| i k \oint_{\partial U} u(\mathbf{y}) g(\mathbf{x}, \mathbf{y}) n_{1} d s(\mathbf{y})-|\mathbf{m}| i k \oint_{\partial U} u(\mathbf{y}) g(\mathbf{x}, \mathbf{y}) n_{1} d s(\mathbf{y}) \\
& -\oint_{\partial U}\left\{g(\mathbf{x}, \mathbf{y}) \nabla_{\mathbf{y}} u(\mathbf{y})\right\} \cdot \mathbf{n} d s(\mathbf{y})+|\mathbf{m}|^{2} \oint_{\partial U} g(\mathbf{x}, \mathbf{y}) \frac{\partial u}{\partial y_{1}}(\mathbf{y}) n_{1} d s(\mathbf{y}) \\
& =\oint_{\partial U}\left\{u(\mathbf{y}) \nabla_{\mathbf{y}} g(\mathbf{x}, \mathbf{y})-g(\mathbf{x}, \mathbf{y}) \nabla_{\mathbf{y}} u(\mathbf{y})\right\} \cdot \mathbf{n} d s(\mathbf{y}) \\
& +|\mathbf{m}|^{2} \oint_{\partial U}\left\{g(\mathbf{x}, \mathbf{y}) \frac{\partial u}{\partial y_{1}}(\mathbf{y})-u(\mathbf{y}) \frac{\partial g}{\partial y_{1}}(\mathbf{x}, \mathbf{y})\right\} n_{1} d s(\mathbf{y}) \\
& -2|\mathbf{m}| i k \oint_{\partial U} u(\mathbf{y}) g(\mathbf{x}, \mathbf{y}) n_{1} d s(\mathbf{y}) \text {. }
\end{aligned}
$$

Proof of Theorem 2.6 [Spectral Theorem for the Neumann-Laplace Operator] Let $\mathcal{A} \subset \mathbb{R}^{d-1}$ satisfy Assumption 2.5. Then there exists an orthonormal system of functions $\left\{\psi_{n}\right\}_{n=0}^{\infty} \subset L^{2}(\mathcal{A})$ and real numbers $\left\{\lambda_{n}\right\}_{n=1}^{\infty} \subset[0, \infty)$ such that

(i) $-\Delta \psi_{n}=\lambda_{n} \psi_{n}$,

(ii) $L^{2}(\mathcal{A})=\overline{\operatorname{span}\left\{\psi_{n}\right\}_{n=0}^{\infty}}$,

(iii) $\left\{\psi_{n}\right\}_{n=0}^{\infty} \subset C^{\infty}(\mathcal{A}) \cap W^{2,2}(\mathcal{A})$,

(iv) $\nabla \psi_{n} \cdot \mathbf{n}=0$ on $\partial \mathcal{A}$ for all $n \in \mathbb{N}_{0}$. 
Proof. Denote by $W^{1,2}(\mathcal{A})$ the Sobolev space (based on weak derivatives) of order $k=1$ with exponent $p=2$ (see e.g. Alt16]). Let $V:=\left\{v \in W^{1,2}(\mathcal{A}) \mid \int_{\mathcal{A}} v(\mathbf{x}) d \mathbf{x}=0\right\}$ denote the subspace of functions with zero mean. Note that $V$ is a closed subspace of the Hilbert space $W^{1,2}(\mathcal{A})$ and thus itself a Hilbert space. Now fix $v \in L^{2}(\mathcal{A})$ and define the bilinear form

$$
a(\cdot, \cdot): V \times V \rightarrow \mathbb{R} \quad a(u, \phi)=\int_{\mathcal{A}} \nabla u \nabla \phi d \mathbf{x}
$$

and the linear form

$$
b_{v}(\cdot): V \rightarrow \mathbb{R} \quad b_{v}(\phi)=\int_{\mathcal{A}} v \phi d \mathbf{x}
$$

By the generalized Poincaré Inequality (see [Alt16, 8.16 p.242])

$$
\|u\|_{L^{2}(\mathcal{A})} \leq C\|\nabla u\|_{L^{2}(\mathcal{A})} \quad \text { for } u \in V
$$

$a(\cdot, \cdot)$ is strictly coercive on $\left(V,\|\cdot\|_{W^{1,2}(\mathcal{A})}\right)$. Hence by the Lax-Milgram theorem for coervice bilinear forms (see e.g. [Eva10, Theorem 1, p.315]), the variational problem

$$
\text { find } u_{v} \in V \text { s.t } \quad a\left(u_{v}, \phi\right)=b_{v}(\phi) \quad \text { for all } \phi \in V,
$$

has a unique solution $u_{v} \in V$. Due to Rellich's theorem (see [Alt16, A8.4 p.261]), the embedding $W^{1,2}(\mathcal{A}) \hookrightarrow L^{2}(\mathcal{A})$ is compact. Hence the operator

$$
-\Delta^{-1}: L^{2}(\mathcal{A}) \rightarrow L^{2}(\mathcal{A}) \quad v \mapsto-\Delta^{-1} v:=u_{v}
$$

is bounded, linear and compact. It is further self-adjoint and positive definite since

$$
\begin{aligned}
\left\langle-\Delta^{-1} v, w\right\rangle_{L^{2}(\mathcal{A})} & =b_{w}\left(-\Delta^{-1} v\right)=a\left(-\Delta^{-1} w,-\Delta^{-1} v\right)=a\left(-\Delta^{-1} v,-\Delta^{-1} w\right) \\
& =b_{v}\left(-\Delta^{-1} w\right)=\left\langle v,-\Delta^{-1} w\right\rangle_{L^{2}(\mathcal{A})} \quad w, v \in L^{2}(\mathcal{A})
\end{aligned}
$$

Thus we can apply the spectral theorem for compact self-adjoint operators (see e.g. [Alt16, $12.12+12.13$ p.395 ff.]). There exist a set of mutually orthogonal eigenfunctions $\left\{\psi_{n}\right\}_{n=1}^{\infty}$ with eigenvalues $\left\{\mu_{n}\right\}_{n=1}^{\infty} \subset\left(0,\left\|-\Delta^{-1}\right\|_{\mathcal{L}\left(L^{2}(\mathcal{A})\right)}\right]$ such that

- $-\Delta^{-1} \psi_{n}=\mu_{n} \psi_{n}$ for all $n \in \mathbb{N}$,

- $\mu_{n} \rightarrow 0$ as $n \rightarrow \infty$,

- $L^{2}(\mathcal{A})=\overline{\operatorname{span}\left\{\psi_{n}\right\}_{n=1}^{\infty}} \oplus \operatorname{ker}\left(-\Delta^{-1}\right)$.

The kernel ker $\left(-\Delta^{-1}\right)$ is given by $\operatorname{span}\left\{\psi_{0}\right\}$ with $\psi_{0}: \equiv \frac{1}{\sqrt{|\mathcal{A}|}}$. Since $\operatorname{ran}\left(-\Delta^{-1}\right) \subset V$ we obtain further that

- $\int_{\mathcal{A}} \psi_{n}(\mathbf{x}) d \mathbf{x}=0$ for all $n \geq 1$. 
In addition, the eigenfunctions solve the elliptic variational problem

$$
\int_{\mathcal{A}} \nabla \psi_{n} \cdot \nabla \phi d \mathbf{x}-\lambda_{n} \int_{\mathcal{A}} \psi_{n} \phi d \mathbf{x}=0 \quad \text { for all } \phi \in W^{1,2}(\mathcal{A})
$$

with

$$
\lambda_{n}=\left\{\begin{array}{ll}
\frac{1}{\mu_{n}} & \text { for } n \geq 1 \\
0 & \text { for } n=0
\end{array} .\right.
$$

Since the right hand side and all coefficients of A.5 are smooth, elliptic interior regularity results (see [GT83, Section 8.3 p.183 ff.]) imply that

- $\left\{\psi_{n}\right\}_{n=1}^{\infty} \subset C^{\infty}(\mathcal{A})$.

The property $\psi_{n} \in W^{2,2}(\mathcal{A})$ is obtained by regularity results up to the boundary (see [Alt16, A12.3 p.415] in case of a smooth boundary and [Gri11, Proof of Theorem 3.2.1.3] in case of a convex domain). Finally we conclude from A.5 and integration by parts that for all $\phi \in C_{c}^{\infty}(\mathcal{A})$

$$
\int_{\mathcal{A}}-\Delta \psi_{n} \phi d \mathbf{x}=\lambda_{n} \int_{\mathcal{A}} \psi_{n} \phi d \mathbf{x}
$$

Thus

$$
-\Delta \psi_{n}=\lambda_{n} \psi_{n}
$$

in the classical sense by the fundamental lemma of calculus of variations [Alt16, 4.22, p.122].

Proposition A.1 (Eigenvalue Asymptotics - Weyl's law).

The eigenvalues from Theorem 2.6 have the following asymptotics

$$
\begin{array}{ll}
\lim _{n \rightarrow \infty} \frac{n}{\lambda_{n}}=\frac{|\mathcal{A}|}{4 \pi} & \text { for } m=2 \\
\lim _{n \rightarrow \infty} \frac{n^{2}}{\lambda_{n}}=\frac{|\mathcal{A}|^{2}}{\pi^{2}} & \text { for } m=1
\end{array}
$$

Proof. For A.6 see Wey12 (original paper from Weyl) or [Str07, Section 11.6 p. 322 ff.] (English version of the proof). For (A.7) the only open, bounded and connected domains are intervals. In that case the eigefunctions are given by

$$
\cos \left(\frac{n \pi}{|\mathcal{A}|} x\right) .
$$

Hence, the eigenvalues are

$$
\lambda_{n}=n^{2}\left(\frac{\pi}{|\mathcal{A}|}\right)^{2} .
$$


Proof of Lemma 2.16 [Series representation for test functions] Assume the eigenfunctions $\psi_{n}$ from Theorem 2.6 satisfy Assumption 2.8. Let further $\phi \in V^{\mathrm{Neu}}=\left\{\phi \in C_{c}^{\infty}(\bar{D}): \frac{\partial \phi}{\partial n}=0\right.$ on $\left.\partial \mathcal{A}\right\}$ and define the coefficients

$$
a_{n}\left(x_{1}\right)=\int_{\mathcal{A}} \phi\left(x_{1}, x_{\perp}\right) \psi_{n}\left(x_{\perp}\right) d x_{\perp} \quad \text { for } n \in \mathbb{N}_{0} .
$$

Then there exists a constant $C$ independent of $n$ such that the derivatives $a_{n}^{(l)}$ up to order 2 satisfy

$$
\max _{l=0,1,2} \sup _{x_{1} \in \mathbb{R}}\left|a_{n}^{(l)}\left(x_{1}\right)\right| \leq C \frac{1}{\lambda_{n}^{3 / 2}} \quad \text { for } n \geq 1
$$

Moreover, we have

(i) for every $\mathbf{x} \in D$ :

$$
\phi(\mathbf{x})=\sum_{n \in \mathbb{N}_{0}} a_{n}\left(x_{1}\right) \psi_{n}\left(x_{\perp}\right)
$$

(ii) for every $x_{1} \in \mathbb{R}$ :

$$
\left[L_{k}^{\top} \phi\right]\left(x_{1}, \cdot\right)=\sum_{n \in \mathbb{N}_{0}}\left[\left(k^{2}-\lambda_{n}\right) a_{n}\left(x_{1}\right)-2|\mathbf{m}| i k a_{n}^{\prime}\left(x_{1}\right)+\beta^{2} a_{n}^{\prime \prime}\left(x_{1}\right)\right] \psi_{n}(\cdot) \quad \text { in } L^{2}(\mathcal{A}) .
$$

Proof. We start with the bound on the coefficients $a_{n}$ and their derivatives up to order 2. Using the variational formulation from the proof of Theorem 2.6 (Equation A.5) and integration by parts we obtain for $n \geq 1$

$$
\begin{aligned}
\left|a_{n}^{(l)}\left(x_{1}\right)\right|= & \left|\int_{\mathcal{A}} \frac{\partial^{(l)}}{\partial x_{1}^{(l)}} \phi\left(x_{1}, x_{\perp}\right) \psi_{n}\left(x_{\perp}\right) d x_{\perp}\right|=\frac{1}{\lambda_{n}}\left|\int_{\mathcal{A}} \nabla_{\perp}\left(\frac{\partial^{(l)}}{\partial x_{1}^{(l)}} \phi\left(x_{1}, x_{\perp}\right)\right) \cdot \nabla_{\perp} \psi_{n}\left(x_{\perp}\right) d x_{\perp}\right| \\
= & \frac{1}{\lambda_{n}} \mid-\int_{\mathcal{A}} \Delta_{\perp} \frac{\partial^{(l)}}{\partial x_{1}^{(l)}} \phi\left(x_{1}, x_{\perp}\right) \psi_{n}\left(x_{\perp}\right) d x_{\perp} \\
& +\oint_{\partial \mathcal{A}} \nabla_{\perp}\left(\frac{\partial^{(l)}}{\partial x_{1}^{(l)}} \phi\left(x_{1}, x_{\perp}\right)\right) \cdot \mathbf{n} \psi_{n}\left(x_{\perp}\right) d s\left(x_{\perp}\right) \mid \\
= & \frac{1}{\lambda_{n}}\left|\int_{\mathcal{A}} \Delta_{\perp} \frac{\partial^{(l)}}{\partial x_{1}^{(l)}} \phi\left(x_{1}, x_{\perp}\right) \psi_{n}\left(x_{\perp}\right) d x_{\perp}\right| \\
= & \frac{1}{\lambda_{n}^{2}}\left|\int_{\mathcal{A}} \nabla_{\perp}\left(\Delta_{\perp} \frac{\partial^{(l)}}{\partial x_{1}^{(l)}} \phi\left(x_{1}, x_{\perp}\right)\right) \cdot \nabla_{\perp} \psi_{n}\left(x_{\perp}\right) d x_{\perp}\right| .
\end{aligned}
$$

Here the boundary integral vanishes since we can interchange the order of differentiation 
of $\phi$ i.e.

$$
\nabla_{\perp}\left(\frac{\partial^{(l)}}{\partial x_{1}^{(l)}} \phi\left(x_{1}, x_{\perp}\right)\right) \cdot \mathbf{n}=\frac{\partial^{(l)}}{\partial x_{1}^{(l)}}\left[\frac{\partial \phi}{\partial n}\left(x_{1}, x_{\perp}\right)\right]
$$

and the inner term vanishes for all $x_{1} \in \mathbb{R}$. Let $d \in\{2,3\}$ denote the spatial dimension, then we obtain from the calculations above

$$
\begin{aligned}
\left|a_{n}^{(l)}\left(x_{1}\right)\right| & \leq \frac{1}{\lambda_{n}^{2}}\|\phi\|_{C^{5}(\bar{D})} \int_{\mathcal{A}} \sum_{j=2}^{d}\left|\frac{\partial \psi_{n}}{\partial x_{j}}\left(x_{\perp}\right)\right| d x_{\perp} \\
& \leq \frac{\sqrt{(d-1)|\mathcal{A}|}}{\lambda_{n}^{2}}\|\phi\|_{C^{5}(\bar{D})} \sqrt{\int_{\mathcal{A}} \sum_{j=2}^{d}\left|\frac{\partial \psi_{n}}{\partial x_{j}}\left(x_{\perp}\right)\right|^{2} d x_{\perp}} \\
& =\frac{\sqrt{(d-1)|\mathcal{A}|}}{\lambda_{n}^{2}}\|\phi\|_{C^{5}(\bar{D})} \sqrt{\lambda_{n}}=\sqrt{(d-1)|\mathcal{A}|}\|\phi\|_{C^{5}(\bar{D})} \frac{1}{\lambda_{n}^{3 / 2}} .
\end{aligned}
$$

Here we have used the Cauchy-Schwarz inequality twice in the second line and the variational formulation (testing with $\psi_{n}$ in Equation (A.5) in the third line. This yields the claim since the upper bound in A.8 is independent of $x_{1}$ and $l$. Now fix $\mathbf{x}=\left(x_{1}, x_{\perp}\right)$, then by standard Fourier theory

$$
\phi\left(x_{1}, \cdot\right)=\sum_{n \in \mathbb{N}_{0}} a_{n}\left(x_{1}\right) \psi_{n}(\cdot) \quad \text { in } L^{2}(\mathcal{A}) .
$$

By the previous statement and the uniform boundedness of the eigenfunctions $\psi_{n}$ (Assumption 2.8), the right hand side converges uniformly on $\mathcal{A}$ i.e. the limit is continuous. As both terms are continuous and they coincide in the $L^{2}$ sense, they have to be identical for every $x_{\perp} \in \mathcal{A}$.

For the last statement we fix $x_{1} \in \mathbb{R}$ and compute the Fourier coefficients of $\left[L_{k}^{\top} \phi\right]\left(x_{1}, \cdot\right)$ with respect to the orthonormal basis $\psi_{n}$ on $L^{2}(\mathcal{A})$. To prove the claim wee need to show that these Fourier coefficients are given by the following identity for all $n \in \mathbb{N}_{0}$

$$
\int_{\mathcal{A}}\left[L_{k}^{\top} \phi\right]\left(x_{1}, x_{\perp}\right) \psi_{n}\left(x_{\perp}\right) d x_{\perp}=\left(k^{2}-\lambda_{n}\right) a_{n}\left(x_{1}\right)-2|\mathbf{m}| i k a_{n}^{\prime}\left(x_{1}\right)+\beta^{2} a_{n}^{\prime \prime}\left(x_{1}\right) .
$$

Firstly note that we can split the differential operator $L_{k}^{\top}$ by

$$
L_{k}^{\top}=D_{k}^{\top}+\Delta_{\perp}
$$

with

$$
D_{k}^{\top}:=k^{2}-2|\mathbf{m}| i k \frac{\partial}{\partial x_{1}}+\beta^{2} \frac{\partial^{2}}{\partial x_{1}^{2}}
$$

Using Green's second formula (cf. [CK13, p. 17]) and the fact that the normal derivative 
of $\phi\left(x_{1}, \cdot\right)$ and $\psi_{n}$ vanishes on $\partial A$ we obtain

$$
\begin{aligned}
\int_{\mathcal{A}}\left[L_{k}^{\top} \phi\right]\left(x_{1}, x_{\perp}\right) \psi_{n}\left(x_{\perp}\right) d x_{\perp} & =D_{k}^{\top} \int_{\mathcal{A}} \phi\left(x_{1}, x_{\perp}\right) \psi_{n}\left(x_{\perp}\right) d x_{\perp}+\int_{\mathcal{A}} \phi\left(x_{1}, x_{\perp}\right) \Delta_{\perp} \psi_{n}\left(x_{\perp}\right) d x_{\perp} \\
& =D_{k}^{\top} \int_{\mathcal{A}} \phi\left(x_{1}, x_{\perp}\right) \psi_{n}\left(x_{\perp}\right) d x_{\perp}-\lambda_{n} \int_{\mathcal{A}} \phi\left(x_{1}, x_{\perp}\right) \psi_{n}\left(x_{\perp}\right) d x_{\perp} \\
& =\left(k^{2}-\lambda_{n}\right) a_{n}\left(x_{1}\right)-2|\mathbf{m}| i k a_{n}^{\prime}\left(x_{1}\right)+\beta^{2} a_{n}^{\prime \prime}\left(x_{1}\right) .
\end{aligned}
$$

This proves A.9 and therefore yields the claim.

Proof of Proposition 4.4 [Convergence of discrete source model] Let $\phi_{n}=1_{\Omega_{n}}\left|\Omega_{n}\right|^{-1 / 2}$ and $q \in C^{0}(\bar{\Omega})$ s.t.

$$
\operatorname{Cov}\left(\pi_{n}, \pi_{n^{\prime}}\right)= \begin{cases}0 & \text { if } n \neq n^{\prime} \\ \frac{1}{\left|\Omega_{n}\right|} \int_{\Omega_{n}} q(\mathbf{y}) d \mathbf{y} & \text { if } n=n^{\prime}\end{cases}
$$

and

$$
\underline{Q}=\sum_{n=1}^{N} \pi_{n} \phi_{n}
$$

Then for all $\mathbf{x}_{m}, \mathbf{x}_{l} \in \mathbb{M}$ we have

$$
\operatorname{Cov}\left(\int_{\Omega} g\left(\mathbf{x}_{m}, \mathbf{y}\right) \underline{Q}(\mathbf{y}) d \mathbf{y}, \int_{\Omega} g\left(\mathbf{x}_{l}, \mathbf{y}\right) \underline{Q}(\mathbf{y}) d \mathbf{y}\right) \rightarrow c_{q}\left(\mathbf{x}_{m}, \mathbf{x}_{l}\right) \quad \text { as } N \rightarrow \infty .
$$

Proof. Evaluation of the left hand side of A.10 yields

$$
\begin{gathered}
\operatorname{Cov}\left(\int_{\Omega} g\left(\mathbf{x}_{m}, \mathbf{y}\right) \underline{Q}(\mathbf{y}) d \mathbf{y}, \int_{\Omega} g\left(\mathbf{x}_{l}, \mathbf{y}\right) \underline{Q}(\mathbf{y}) d \mathbf{y}\right) \\
=\sum_{n=1}^{N}\left\{\left|\Omega_{n}\right|^{-1}\left[\int_{\Omega_{n}} g\left(\mathbf{x}_{m}, \mathbf{y}\right) d \mathbf{y}\right]\left[\int_{\Omega_{n}} \overline{g\left(\mathbf{x}_{l}, \mathbf{y}\right)} d \mathbf{y}\right]\left|\Omega_{n}\right|^{-1} \int_{\Omega_{n}} q(\mathbf{y}) d \mathbf{y}\right\} .
\end{gathered}
$$

For the real part we obtain

$$
\begin{gathered}
\operatorname{Re}\left(\operatorname{Cov}\left(\int_{\Omega} g\left(\mathbf{x}_{m}, \mathbf{y}\right) \underline{Q}(\mathbf{y}) d \mathbf{y}, \int_{\Omega} g\left(\mathbf{x}_{l}, \mathbf{y}\right) \underline{Q}(\mathbf{y}) d \mathbf{y}\right)\right) \\
=\sum_{n=1}^{N}\left\{\left|\Omega_{n}\right|^{-1}\left[\int_{\Omega_{n}} \operatorname{Re}\left(g\left(\mathbf{x}_{m}, \mathbf{y}\right)\right) d \mathbf{y}\right]\left[\int_{\Omega_{n}} \operatorname{Re}\left(g\left(\mathbf{x}_{l}, \mathbf{y}\right)\right) d \mathbf{y}\right]\left|\Omega_{n}\right|^{-1} \int_{\Omega_{n}} q(\mathbf{y}) d \mathbf{y}\right. \\
\left.+\left|\Omega_{n}\right|^{-1}\left[\int_{\Omega_{n}} \operatorname{Im}\left(g\left(\mathbf{x}_{m}, \mathbf{y}\right)\right) d \mathbf{y}\right]\left[\int_{\Omega_{n}} \operatorname{Im}\left(g\left(\mathbf{x}_{l}, \mathbf{y}\right)\right) d \mathbf{y}\right]\left|\Omega_{n}\right|^{-1} \int_{\Omega_{n}} q(\mathbf{y}) d \mathbf{y}\right\} .
\end{gathered}
$$


Now we can apply the mean value theorem of integral calculus for each of the integrals over $\Omega_{n}$ i.e. there exist $\mathbf{y}_{n}^{(1)}, \mathbf{y}_{n}^{(2)}, \mathbf{y}_{n}^{(3)}, \mathbf{y}_{n}^{(4)}, \mathbf{y}_{n}^{(5)} \in \Omega_{n}$ such that

$$
\begin{aligned}
\sum_{n=1}^{N}\{\ldots\}= & \sum_{n=1}^{N}\left|\Omega_{n}\right| \operatorname{Re}\left(g\left(\mathbf{x}_{m}, \mathbf{y}_{n}^{(1)}\right)\right) \operatorname{Re}\left(g\left(\mathbf{x}_{l}, \mathbf{y}_{n}^{(2)}\right)\right) q\left(\mathbf{y}_{n}^{(3)}\right) \\
& +\sum_{n=1}^{N}\left|\Omega_{n}\right| \operatorname{Im}\left(g\left(\mathbf{x}_{m}, \mathbf{y}_{n}^{(4)}\right)\right) \operatorname{Im}\left(g\left(\mathbf{x}_{l}, \mathbf{y}_{n}^{(5)}\right)\right) q\left(\mathbf{y}_{n}^{(3)}\right) .
\end{aligned}
$$

Recall the definition of the correlation kernel

$$
\begin{aligned}
& \operatorname{Re}\left(c_{q}\left(\mathbf{x}_{m}, \mathbf{x}_{l}\right)\right)=\operatorname{Re}\left(\int_{\Omega} g\left(\mathbf{x}_{m}, \mathbf{y}\right) \overline{g\left(\mathbf{x}_{l}, \mathbf{y}\right)} q(\mathbf{y}) d \mathbf{y}\right) \\
& =\int_{\Omega} \operatorname{Re}\left(g\left(\mathbf{x}_{m}, \mathbf{y}\right)\right) \operatorname{Re}\left(g\left(\mathbf{x}_{l}, \mathbf{y}\right)\right) q(\mathbf{y}) d \mathbf{y}+\int_{\Omega} \operatorname{Im}\left(g\left(\mathbf{x}_{m}, \mathbf{y}\right)\right) \operatorname{Im}\left(g\left(\mathbf{x}_{l}, \mathbf{y}\right)\right) q(\mathbf{y}) d \mathbf{y} .
\end{aligned}
$$

The functions $g\left(\mathbf{x}_{m}, \cdot\right), g\left(\mathbf{x}_{l}, \cdot\right), q$ are uniformly continuous on $\Omega$ since they are continuous and $\bar{\Omega}$ is compact. Hence, for any $\epsilon>0$ there exists an $N_{\epsilon} \in \mathbb{N}$ such that for all $N \geq N_{\epsilon}$, every $n=1, \ldots, N$ and $\mathbf{y} \in \Omega$

$$
\begin{aligned}
f_{n}(\mathbf{y}):= & \mid \operatorname{Re}\left(g\left(\mathbf{x}_{m}, \mathbf{y}_{n}^{(1)}\right)\right) \operatorname{Re}\left(g\left(\mathbf{x}_{l}, \mathbf{y}_{n}^{(2)}\right)\right) q\left(\mathbf{y}_{n}^{(3)}\right) \\
& +\operatorname{Im}\left(g\left(\mathbf{x}_{m}, \mathbf{y}_{n}^{(4)}\right)\right) \operatorname{Im}\left(g\left(\mathbf{x}_{l}, \mathbf{y}_{n}^{(5)}\right)\right) q\left(\mathbf{y}_{n}^{(3)}\right) \\
& -\operatorname{Re}\left(g\left(\mathbf{x}_{m}, \mathbf{y}\right)\right) \operatorname{Re}\left(g\left(\mathbf{x}_{l}, \mathbf{y}\right)\right) q(\mathbf{y})-\operatorname{Im}\left(g\left(\mathbf{x}_{m}, \mathbf{y}\right)\right) \operatorname{Im}\left(g\left(\mathbf{x}_{l}, \mathbf{y}\right)\right) q(\mathbf{y}) \mid \leq \epsilon .
\end{aligned}
$$

Finally, by the last equation, we can bound the discretization error

$$
\begin{aligned}
& \left|\operatorname{Re}\left(\operatorname{Cov}\left(\int_{\Omega} g\left(\mathbf{x}_{m}, \mathbf{y}\right) \underline{Q}(\mathbf{y}) d \mathbf{y}, \int_{\Omega} g\left(\mathbf{x}_{l}, \mathbf{y}\right) \underline{Q}(\mathbf{y}) d \mathbf{y}\right)\right)-\operatorname{Re}\left(c_{q}\left(\mathbf{x}_{m}, \mathbf{x}_{l}\right)\right)\right| \\
\leq & \sum_{n=1}^{N} \int_{\Omega_{n}} f_{n}(\mathbf{y}) d \mathbf{y} \leq|\Omega| \epsilon .
\end{aligned}
$$

Since $\epsilon$ can be chosen arbitrarily small this yields

$$
\operatorname{Re}\left(\operatorname{Cov}\left(\int_{\Omega} g\left(\mathbf{x}_{m}, \mathbf{y}\right) \underline{Q}(\mathbf{y}) d \mathbf{y}, \int_{\Omega} g\left(\mathbf{x}_{l}, \mathbf{y}\right) \underline{Q}(\mathbf{y}) d \mathbf{y}\right)\right) \rightarrow \operatorname{Re}\left(c_{q}\left(\mathbf{x}_{m}, \mathbf{x}_{l}\right)\right) \quad \text { as } N \rightarrow \infty .
$$

The proof for the imaginary part is completely analogous and will be omitted here. 


\section{Appendix}

\section{Fundamentals from Calculus and Convex Analysis}

The theorem of Fubini-Tonelli is a version of Fubini's theorem that does not require the assumption of integrability with respect to the product measure. Since it is used several times within this thesis it is included in this appendix. For further reading on the fundamentals of measure theory, we refer to one of the numerous monographs on this subject (such as [Els11]).

Theorem B.1 (Fubini-Tonelli cf. [Els11] [2.1 c) p.176]).

Let $(X, \mu),(Y, \nu)$ be measure spaces with $\sigma$-finite measures and $f$ a function that is measurable with respect to the product measure $\mu \otimes \nu$. Assume that one of the following iterated integrals exists

$$
\begin{aligned}
& \int_{X}\left(\int_{Y}|f(x, y)| d \nu(y)\right) d \mu(x) \\
& \int_{Y}\left(\int_{X}|f(x, y)| d \mu(x)\right) d \nu(y) .
\end{aligned}
$$

Then we have

$$
\int_{X \times Y} f d \mu \otimes \nu=\int_{X}\left(\int_{Y} f(x, y) d \nu(y)\right) d \mu(x)=\int_{Y}\left(\int_{X} f(x, y) d \mu(x)\right) d \nu(y) .
$$

The class of Hilbert-Schmidt operators between two Hilbert spaces $\mathcal{H}_{1}, \mathcal{H}_{2}$ is a subset of all linear compact operators $K: \mathcal{H}_{1} \rightarrow \mathcal{H}_{2}$ and can be defined by means of the singular values of $K$. However, for this work a definition on separable Hilbert spaces is sufficient.

Definition \& Proposition B.2 (Hilbert-Schmidt operator).

Let $\mathcal{H}_{1}, \mathcal{H}_{2}$ be separable Hilbert spaces i.e. there exist complete orthonormal bases $\left\{e_{n}\right\}_{n \in \mathbb{N}} \subset$ $\mathcal{H}_{1}$ and $\left\{f_{n}\right\}_{n \in \mathbb{N}} \subset \mathcal{H}_{2}$. Let $T: \mathcal{H}_{1} \rightarrow \mathcal{H}_{2}$ be a linear bounded operator, then $T$ is called 
Hilbert-Schmidt operator if it satisfies

$$
\sum_{n \in \mathbb{N}}\left\|T e_{n}\right\|_{\mathcal{H}_{2}}^{2}<\infty
$$

In particular $T$ is compact. The Hilbert-Schmidt norm of $T$ is defined as

$$
\|T\|_{\mathrm{HS}}:=\left(\sum_{n \in \mathbb{N}}\left\|T e_{n}\right\|_{\mathcal{H}_{2}}^{2}\right)^{1 / 2},
$$

where the value of the series is independent of the choice of the orthonormal basis. The space of Hilbert-Schmidt operators $T: \mathcal{H}_{1} \rightarrow \mathcal{H}_{2}$ will be denoted by $\mathrm{HS}\left(\mathcal{H}_{1}, \mathcal{H}_{2}\right)$ (or $\mathrm{HS}\left(\mathcal{H}_{1}\right)$ in case of $\left.\mathcal{H}_{1}=\mathcal{H}_{2}\right)$. Moreover, $\mathrm{HS}\left(\mathcal{H}_{1}, \mathcal{H}_{2}\right)$ is a Hilbert space with scalar product

$$
\langle S, T\rangle_{\mathrm{HS}}:=\sum_{n \in \mathbb{N}}\left\langle S e_{n}, T e_{n}\right\rangle_{\mathcal{H}_{2}}
$$

and the definition is again independent of the choice of the orthonormal basis.

Proof. The definition and proofs of this statement can be found in various textbooks on operator theory. We refer to [Sun16], where the statements can be found in Definition 3.3.2, Lemma 3.3.1. (independence of the basis), Proposition 3.3.3. c) (compactness) and Remark 3.3.4 (Hilbert space with scalar product).

For $L^{2}$ spaces there exists a characterization of Hilbert-Schmidt operators by integral operators with square integrable kernel.

Proposition B.3 (Hilbert-Schmidt integral operator cf. [Sun16, Proposition 3.3.5]). Let $\left(X, \mathcal{B}_{X}, \mu\right)$ and $\left(Y, \mathcal{B}_{Y}, \nu\right)$ be $\sigma$-finite measure spaces and let $\mathcal{H}_{1}=L^{2}\left(X, \mathcal{B}_{X}, \mu\right)$, $\mathcal{H}_{2}=L^{2}\left(Y, \mathcal{B}_{Y}, \nu\right)$. For a bounded linear operator $T: \mathcal{H}_{1} \rightarrow \mathcal{H}_{2}$ the following statements are equivalent

(i) $T \in \operatorname{HS}\left(\mathcal{H}_{1}, \mathcal{H}_{2}\right)$.

(ii) There exists a kernel $k \in L^{2}\left(X \times Y, \mathcal{B}_{X} \otimes \mathcal{B}_{Y}, \mu \otimes \nu\right)$ such that for all $f \in \mathcal{H}_{1}$

$$
(T f)(x)=\int_{Y} k(x, y) f(y) d \nu(y) \quad \text { almost everywhere. }
$$

Moreover, if one of these statements is satisfied we have

$$
\|T\|_{\mathrm{HS}}=\|k\|_{L^{2}(\mu \times \nu)} .
$$

For the definition of real analytic functions we employ the multi-index notation. Let 
$\alpha \in \mathbb{N}_{0}^{d}$ and $\mathbf{z} \in \mathbb{R}^{d}$, then the power with respect to a multi-index is defined as

$$
\mathbf{z}^{\alpha}:=\prod_{j=1}^{d} z_{j}^{\alpha_{j}} .
$$

Definition B.4 (Real analytic function cf. [KP02, Definition 2.2.1, p.29]).

Let $U \subset \mathbb{R}^{d}$ be an open set. A function $f: U \rightarrow \mathbb{C}$ is called real analytic if for each $\mathbf{y} \in U$ there exists a neighborhood $O_{\mathbf{y}}$ of $\mathbf{y}$ such that there exists a power series expansion with coefficients $c_{\alpha} \in \mathbb{C}$ that satisfies

$$
f(\mathbf{x})=\sum_{\alpha \in \mathbb{N}_{0}^{d}} c_{\alpha}(\mathbf{x}-\mathbf{y})^{\alpha} \quad \text { for } \mathbf{x} \in O_{\mathbf{y}} .
$$

Definition B.5 (Convex function).

Let $\mathbb{X}$ be a vector space. A function $\mathcal{F}: \mathbb{X} \rightarrow[-\infty, \infty]$ is called convex if for any $\lambda \in(0,1)$ and $x_{1}, x_{2} \in \mathbb{X}$, the inequality

$$
\mathcal{F}\left(\lambda x_{1}+(1-\lambda) x_{2}\right) \leq \lambda \mathcal{F}\left(x_{1}\right)+(1-\lambda) \mathcal{F}\left(x_{2}\right)
$$

holds true. $\mathcal{F}$ is called strictly convex if the inequality is strict for $x_{1} \neq x_{2}$.

For this work it is sufficient to use the the definition of sequential lower semicontinuity. For a definition on lower semcontinuity on topological vector spaces using nets we refer to [BC17, Chapter 1].

Definition B.6 (Sequentially lower semicontinuous function).

Let $(\mathbb{X}, \tau)$ be a Hausdorff space. A function $\mathcal{F}: \mathbb{X} \rightarrow[-\infty, \infty]$ is called sequentially lower semicontinuous at $x \in \mathbb{X}$ if for every sequence $x_{n} \stackrel{\tau}{\rightarrow} x$,

$$
\mathcal{F}(x) \leq \liminf _{n \rightarrow \infty} \mathcal{F}\left(x_{n}\right) .
$$

$\mathcal{F}$ is called sequentially lower semicontinuous on $\mathbb{X}$ if $\mathcal{F}$ is sequentially lower semicontinuous for all $x \in \mathbb{X}$. 



\section{Bibliography}

[AF03] R. Adams and J. J. F. Fournier. Sobolev Spaces. Elsevier LTD, Oxford, 2003 .

[Ahl13] T. Ahlefeldt. Aeroacoustic measurements of a scaled half-model at high reynolds numbers. AIAA Journal, 51(12):2783-2791, December 2013.

[Ahl16] T. Ahlefeldt. Microphone array measurement in european transonic wind tunnel at flight reynolds numbers. AIAA Journal, 55(1):36-48, jan 2016.

[AHSE95] H. H. Andersen, M. Højbjerre, D. Sørensen, and P. S. Eriksen. Linear and Graphical Models. Springer New York, 1995.

[Ait36] A. C. Aitken. IV.- on least squares and linear combination of observations. Proceedings of the Royal Society of Edinburgh, 55:42-48, 1936.

[Alt16] H. W. Alt. Linear Functional Analysis. Springer London, 2016.

[Ami78] R. K. Amiet. Refraction of sound by a shear layer. Journal of Sound and Vibration, 58(4):467-482, June 1978.

[Ant12] J. Antoni. A Bayesian approach to sound source reconstruction: Optimal basis, regularization, and focusing. The Journal of the Acoustical Society of America, 131(4):2873-2890, April 2012.

[BC17] H. H. Bauschke and P. L. Combettes. Convex Analysis and Monotone Operator Theory in Hilbert Spaces. Springer International Publishing, 2017.

[BE04] D. Blacodon and G. Elias. Level estimation of extended acoustic sources using a parametric method. Journal of Aircraft, 41(6):1360-1369, November 2004 .

[Bec17] A. Beck. First-Order Methods in Optimization. Society for Industrial and Applied Mathematics, oct 2017.

[Ben17] Benchmarking array analysis methods. https://www.b-tu.de/ fg-akustik/lehre/aktuelles/arraybenchmark, 2017. 
[BH06] T. F. Brooks and W. M. Humphreys. A deconvolution approach for the mapping of acoustic sources (DAMAS) determined from phased microphone arrays. Journal of Sound and Vibration, 294(4-5):856-879, 2006.

$\left[\mathrm{BHE}^{+} 17\right]$ C. J. Bahr, W.M. Humphreys, D. Ernst, T. Ahlefeldt, C. Spehr, A. Pereira, Q. Leclère, C. Picard, R. Porteous, D. Moreau, J. R. Fischer, and C. J. Doolan. A comparison of microphone phased array methods applied to the study of airframe noise in wind tunnel testing. In $23^{\text {rd }}$ AIAA/CEAS Aeroacoustics Conference, Reston, VA, June 2017. American Institute of Aeronautics and Astronautics.

[BHMR07] N. Bissantz, T. Hohage, A. Munk, and F. Ruymgaart. Convergence rates of general regularization methods for statistical inverse problems and applications. SIAM Journal on Numerical Analysis, 45(6):2610-2636, jan 2007.

[BK76] J. Billingsley and R. Kinns. The acoustic telescope. Journal of Sound and Vibration, 48(4):485-510, October 1976.

[BKP87] B. Barsikow, W. F. King, and E. Pfizenmaier. Wheel/rail noise generated by a high-speed train investigated with a line array of microphones. Journal of Sound and Vibration, 118(1):99-122, oct 1987.

[BL20] B. Bai and X. Li. Acoustic sources mapping based on the non-negative 11/2 regularization. Applied Acoustics, 169:107456, dec 2020.

[BMP87] T. F. Brooks, M. A. Marcolini, and D. S. Pope. A directional array approach for the measurement of rotor noise source distributions with controlled spatial resolution. Journal of Sound and Vibration, 112(1):192197, jan 1987.

[BPT05] L. Borcea, G. Papanicolaou, and C. Tsogka. Interferometric array imaging in clutter. Inverse Problems, 21(4):1419-1460, July 2005.

[BSC01] N. Bleistein, J. W. Stockwell, and J. K. Cohen. Mathematics of Multidimensional Seismic Imaging, Migration, and Inversion. Springer New York, 2001.

[BT09] A. Beck and M. Teboulle. A fast iterative shrinkage-thresholding algorithm for linear inverse problems. SIAM Journal on Imaging Sciences, 2(1):183-202, jan 2009.

[Cap69] J. Capon. High-resolution frequency-wavenumber spectrum analysis. Proceedings of the IEEE, 57(8):1408-1418, 1969.

[CC06] F. Cakoni and D. Colton. Qualitative Methods in Inverse Scattering Theory. Springer-Verlag, 2006. 
[Cha00] C. J. Chapman. Similarity variables for sound radiation in a uniform flow. Journal of Sound and Vibration, 233(1):157-164, May 2000.

[CK13] D. Colton and R. Kress. Inverse Acoustic and Electromagnetic Scattering Theory. Springer New York, 3rd edition, 2013.

[CZO87] H. Cox, R. Zeskind, and M. Owen. Robust adaptive beamforming. IEEE Transactions on Acoustics, Speech, and Signal Processing, 35(10):13651376 , oct 1987 .

[DES20] J. Dölz, H. Egger, and M. Schlottbom. A model reduction approach for inverse problems with operator valued data. arXiv:2004.11827 [cs, math], April 2020.

$\left[\mathrm{EGK}^{+} 20\right]$ D. Ernst, R. Geisler, T. Kleindienst, T. Ahlefeldt, and C. Spehr. Portable 512 mems-microphone-array for 3d-intensity- and beamformingmeasurements using an fpga based data-acquisition-system. In 8th Berlin Beamforming Conference. GFaI, Gesellschaft zu Förderung angewandter Informatik e.V., Berlin, March 2020.

[EHN96] H. W. Engl, M. Hanke, and A. Neubauer. Regularization of Inverse Problems. Springer Netherlands, 1996.

[Els11] J. Elstrodt. Maß- und Integrationstheorie. Springer Berlin Heidelberg, 2011.

[ESB15] D. Ernst, C. Spehr, and T. Berkefeld. Decorrelation of acoustic wave propagation through the shear layer in open jet wind tunnel. In 21st AIAA/CEAS Aeroacoustics Conference. American Institute of Aeronautics and Astronautics, jun 2015.

[Eva10] L. C. Evans. Partial Differential Equations. American Mathematical Society, 2010.

[FGHB14] D. Fournier, L. Gizon, T. Hohage, and A. C. Birch. Generalization of the noise model for time-distance helioseismology. Astronomy \& Astrophysics, 567:A137, jul 2014.

[Fol95] G. B. Folland. Introduction to Partial Differential Equations. Princeton University Press, 1995.

[Fra70] J. N. Franklin. Well-posed stochastic extensions of ill-posed linear problems. Journal of Mathematical Analysis and Applications, 31(3):682-716, sep 1970.

[GB04] L. Gizon and A. C. Birch. Time-distance helioseismology: Noise estimation. The Astrophysical Journal, 614(1):472-489, oct 2004. 
[GFY $\left.{ }^{+} 18\right] \quad$ L. Gizon, D. Fournier, D. Yang, A. C. Birch, and H. Barucq. Signal and noise in helioseismic holography. Astronomy $\&$ Astrophysics, 620:A136, $\operatorname{dec} 2018$.

[GHR12] R. Griesmaier, M. Hanke, and T. Raasch. Inverse Source Problems for the Helmholtz Equation and the Windowed Fourier Transform. SIAM Journal on Scientific Computing, 34(3):A1544-A1562, jan 2012.

[GHR13] R. Griesmaier, M. Hanke, and T. Raasch. Inverse Source Problems for the Helmholtz Equation and the Windowed Fourier Transform II. SIAM Journal on Scientific Computing, 35(5):A2188-A2206, jan 2013.

[GJW75] F.-R. Grosche, J. H. Jones, and G. A. Wilhold. Measurements of the distribution of sound source intensities in turbulent jets. In Aeroacoustics: Jet and Combustion Noise; Duct Acoustics, pages 79-92. American Institute of Aeronautics and Astronautics, New York, jan 1975.

[GKK18] S. Gombots, M. Kaltenbacher, and B. Kaltenbacher. Inverse scheme for acoustic source localization in 3d. In EURONOISE2018, Crete, 2018. Michael Taroudakis.

[GP17] A. Ganguly and I. Panahi. Non-uniform microphone arrays for robust speech source localization for smartphone-assisted hearing aid devices. Journal of Signal Processing Systems, 90(10):1415-1435, nov 2017.

[Gri11] P. Grisvard. Elliptic Problems in Nonsmooth Domains. Society for Industrial and Applied Mathematics, jan 2011.

[GS16] R. Griesmaier and J. Sylvester. Far field splitting by iteratively reweighted $\ell^{1}$ minimization. SIAM Journal on Applied Mathematics, 76(2):705-730, jan 2016.

[GS17a] R. Griesmaier and J. Sylvester. Uncertainty principles for inverse source problems, far field splitting, and data completion. SIAM Journal on Applied Mathematics, 77(1):154-180, jan 2017.

[GS17b] R. Griesmaier and J. Sylvester. Uncertainty principles for threedimensional inverse source problems. SIAM Journal on Applied Mathematics, 77(6):2066-2092, jan 2017.

[GSAL15] A. L. Gregory, S. Sinayoko, A. Agarwal, and J. Lasenby. An acoustic space-time and the lorentz transformation in aeroacoustics. International Journal of Aeroacoustics, 14(7):977-1003, nov 2015.

[GT83] D. Gilbarg and N. S. Trudinger. Elliptic Partial Differential Equations of Second Order. Springer, 2nd edition, 1983.

[Han10] P. C. Hansen. Discrete Inverse Problems. Society for Industrial and Applied Mathematics, jan 2010. 
[HHL $\left.{ }^{+} 93\right] \quad$ S. Haykin, T. V. Ho, J. Litva, J. G. McWhirter, A. Nehorai, U. Nickel, B. Ottersten, T. J. Shepherd, B.D. Steinberg, P. Stoica, M. Viberg, and Z. Zhu. Radar Array Processing. Springer Berlin Heidelberg, 1993.

[HJ91] R. A. Horn and C. R. Johnson. Topics in Matrix Analysis. Cambridge University Press, apr 1991.

[HK21] K. Van Huynh and B. Kaltenbacher. Some application examples of minimization based formulations of inverse problems and their regularization. Inverse Problems \& Imaging, 15(3):415, 2021.

[Hög74] J. A. Högbom. Aperture synthesis with a non-regular distribution of interferometer baselines. Astronomy and Astrophysics Supplement Series, 15:417-426, 1974.

[HRS20] T. Hohage, H.-G. Raumer, and C. Spehr. Uniqueness of an inverse source problem in experimental aeroacoustics. Inverse Problems, mar 2020.

[HS17] G. Herold and E. Sarradj. Performance analysis of microphone array methods. Journal of Sound and Vibration, 401:152-168, aug 2017.

[HW15] T. Hohage and F. Weidling. Verification of a variational source condition for acoustic inverse medium scattering problems. Inverse Problems, 31(7):075006, jun 2015.

[Int97] International Organization for Standardization. Acoustics - preferred frequencies. https://www.iso.org/standard/1350.html, 1997.

[Iss16] L. Isserlis. On certain probable errors and correlation coefficients of multiple frequency distrubutions with skew regression. Biometrika, 11(3):185$190,1916$.

[JBJM98] W. Humphreys Jr., T. Brooks, W. Hunter Jr., and K. Meadows. Design and use of microphone directional arrays for aeroacoustic measurements. In 36th AIAA Aerospace Sciences Meeting and Exhibit. American Institute of Aeronautics and Astronautics, jan 1998.

[JD93] D. H. Johnson and D. E. Dudgeon. Array Signal Processing. P T R Prentice Hall, Englewood Cliffs, NJ, 1993.

[Joh82] F. John. Partial Differential Equations. Springer New York, 4th edition, 1982.

[Kal16] B. Kaltenbacher. Regularization based on all-at-once formulations for inverse problems. SIAM Journal on Numerical Analysis, 54(4):2594-2618, jan 2016. 
[Kal18] B. Kaltenbacher. Minimization based formulations of inverse problems and their regularization. SIAM Journal on Optimization, 28(1):620-645, jan 2018.

[Kar04] T. Kariya. Generalized least squares. John Wiley \& Sons, Chichester, West Sussex Hoboken, NJ, 2004.

[KG07] A. Kirsch and N. Grinberg. The Factorization Method for Inverse Problems. Oxford University Press, dec 2007.

[Kir98] A. Kirsch. Characterization of the shape of a scattering obstacle using the spectral data of the far field operator. Inverse Problems, 14(6):1489-1512, dec 1998.

[Kir99] A. Kirsch. Factorization of the far-field operator for the inhomogeneous medium case and an application in inverse scattering theory. Inverse Problems, 15(2):413-429, jan 1999.

[Kir11] A. Kirsch. An Introduction to the Mathematical Theory of Inverse Problems. Springer New York, 2011.

[KKG18] M. Kaltenbacher, B. Kaltenbacher, and S. Gombots. Inverse scheme for acoustic source localization using microphone measurements and finite element simulations. Acta Acustica united with Acustica, 104(4):647-656, jul 2018.

[KP02] S. G. Krantz and H. R. Parks. A Primer of Real Analytic Functions. Birkhäuser Boston, 2002.

[LB00] C. Lindsey and D. C. Braun. Basic principles of solar acoustic holography - (invited review). Solar Physics, 192(1/2):261-284, 2000.

[LCH+19] T. Long, J. Chen, G. Huang, J. Benesty, and I. Cohen. Acoustic source localization based on geometric projection in reverberant and noisy environments. IEEE Journal of Selected Topics in Signal Processing, 13(1):143155, mar 2019.

[Lep90] O. V. Lepskii. On a problem of adaptive estimation in Gaussian white noise. Theory of Probability \& Its Applications, 35(3):454-466, jan 1990.

[Lig52] M. J. Lighthill. On sound generated aerodynamically. i. general theory. Proceedings of the Royal Society A: Mathematical, Physical and Engineering Sciences, 211(1107):564-587, mar 1952.

[Lig54] M. J. Lighthill. On sound generated aerodynamically II. turbulence as a source of sound. Proceedings of the Royal Society of London. Series A. Mathematical and Physical Sciences, 222(1148):1-32, feb 1954. 
[Lin00] C. Lindsey. Seismic images of the far side of the sun. Science, 287(5459):1799-1801, mar 2000.

$\left[\mathrm{LPB}^{+} 17\right] \quad$ Q. Leclère, A. Pereira, C. Bailly, J. Antoni, and C. Picard. A unified formalism for acoustic imaging based on microphone array measurements. International Journal of Aeroacoustics, 16(4-5):431-456, jul 2017.

[LPS89] M. S. Lehtinen, L. Paivarinta, and E. Somersalo. Linear inverse problems for generalised random variables. Inverse Problems, 5(4):599-612, aug 1989.

[Mah36] P. C. Mahalanobis. On the generalised distance in statistics. In Proceedings National Institute of Science, India, volume 2, pages 49-55, 1936.

[Mat20] Mathematisches Forschungsinstitut Oberwolfach. Computational inverse problems for partial differential equations. Technical report, 2020.

[ML18] W. Ma and X. Liu. Compression computational grid based on functional beamforming for acoustic source localization. Applied Acoustics, 134:7587, may 2018.

[MMSS $\left.{ }^{+} 19\right] \quad$ R. Merino-Martínez, P. Sijtsma, M. Snellen, T. Ahlefeldt, J. Antoni, C. J. Bahr, D. Blacodon, D. Ernst, A. Finez, S. Funke, T.F. Geyer, S. Haxter, G. Herold, X. Huang, W. M. Humphreys, Q. Leclère, A. Malgoezar, U. Michel, T. Padois, A. Pereira, C. Picard, E. Sarradj, H. Siller, D. G. Simons, and C. Spehr. A review of acoustic imaging methods using phased microphone arrays. CEAS Aeronautical Journal, 10:197-230, mar 2019.

[Mor66] V. A. Morozov. On the solution of functional equations by the method of regularization. Soviet Math. Dokl., 7:414-417, 1966.

[MP06] P. Mathé and S. V. Pereverzev. Regularization of some linear ill-posed problems with discretized random noisy data. Mathematics of Computation, 75(256):1913-1929, jun 2006.

[MST12] G. L. Mazzieri, R. D. Spies, and K. G. Temperini. Existence, uniqueness and stability of minimizers of generalized Tikhonov-Phillips functionals. Journal of Mathematical Analysis and Applications, 396(1):396-411, dec 2012 .

[OSL07] S. Oerlemans, P. Sijtsma, and B. Méndez López. Location and quantification of noise sources on a wind turbine. Journal of Sound and Vibration, 299(4-5):869-883, feb 2007.

[PAL15] A. Pereira, J. Antoni, and Q. Leclère. Empirical Bayesian regularization of the inverse acoustic problem. Applied Acoustics, 97:11-29, oct 2015.

[PJS14] L. L. Scharf P. J. Schreier. Statistical Signal Processing of Complex-Valued Data. Cambridge University Press, 2014. 
[RAH12] C. R. Johnson R. A. Horn. Matrix Analysis. Cambridge University Press, 2012.

[RS75] M. Reed and B. Simon. Methods of Modern Mathematical Physics. II: Fourier Analysis, Self-Adjointness. Academic Press, 1975.

[RSHE20] H.-G. Raumer, C. Spehr, T. Hohage, and D. Ernst. Weighted data spaces for correlation-based array imaging in experimental aeroacoustics. Journal of Sound and Vibration, page 115878, nov 2020.

[Sch86] R. Schmidt. Multiple emitter location and signal parameter estimation. IEEE Transactions on Antennas and Propagation, 34(3):276-280, mar 1986.

[SGG $\left.{ }^{+} 09\right]$ O. Scherzer, M. Grasmair, H. Grossauer, Markus M. Haltmeier, and F. Lenzen. Variational Methods in Imaging. Springer New York, New York, 2009.

[Sij04] P. Sijtsma. Experimental techniques for identification and characterisation of noise sources. Technical Report NLR-TP-2004-165, National Aerospace Laboratory NLR, 2004.

[Sij07] P. Sijtsma. CLEAN based on spatial source coherence. International Journal of Aeroacoustics, 6(4):357-374, dec 2007.

[Sij10] P. Sijtsma. Phased array beamforming applied to wind tunnel and fly-over tests. Technical Report NLR-TP-2010-549, National Aerospace Laboratory NLR, 2010.

[SKHK12] T. Schuster, B. Kaltenbacher, B. Hofmann, and K. S. Kazimierski. Regularization Methods in Banach Spaces. DE GRUYTER, jul 2012.

[Som49] A. Sommerfeld. Partial differential equations in physics, volume 1. Academic press, 1949.

[SS06] E. Sarradj and C. Schulze. Practical application of orthogonal beamforming. In Euronoise, 2006.

[Str07] W. A. Strauss. Partial Differential Equations: An Introduction. Wiley, 2nd edition, 2007.

[Sun97] V. S. Sunder. Functional analysis : spectral theory. Birkhäuser Verlag, Basel Boston, 1997.

[Sun16] V. S. Sunder. Operators on Hilbert Space. Springer Singapore, 2016.

[Suz08] T. Suzuki. Generalized inverse beam-forming algorithm resolving coherent/incoherent, distributed and multipole sources. In 14th $A I A A / C E A S$ Aeroacoustics Conference (29th AIAA Aeroacoustics Conference). American Institute of Aeronautics and Astronautics, may 2008. 
[Tes12] G. Teschl. Ordinary Differential Equations and Dynamical Systems. American Mathematical Society, aug 2012.

[VB88] B. D. Van Veen and K. M. Buckley. Beamforming: a versatile approach to spatial filtering. IEEE ASSP Magazine, 5(2):4-24, apr 1988.

[Wei19] F. Weidling. Variational Source Conditions and Conditional Stability Estimates for Inverse Problems in PDEs. Göttingen University Press, 2019.

[Wel67] P. Welch. The use of fast fourier transform for the estimation of power spectra: A method based on time averaging over short, modified periodograms. IEEE Transactions on Audio and Electroacoustics, 15(2):7073, jun 1967.

[Wey12] H. Weyl. Das asymptotische Verteilungsgesetz der Eigenwerte linearer partieller Differentialgleichungen (mit einer Anwendung auf die Theorie der Hohlraumstrahlung). Mathematische Annalen, 71(4):441-479, dec 1912 .

[WH12] F. Werner and T. Hohage. Convergence rates in expectation for Tikhonovtype regularization of inverse problems with Poisson data. Inverse Problems, 28(10):104004, oct 2012.

[WH19] F. Werner and B. Hofmann. Convergence analysis of (statistical) inverse problems under conditional stability estimates. Inverse Problems, 36(1):015004, dec 2019.

[WTdHEB09] J. Wind, E. Tijs, and de H.-E. Bree. Source localization using acoustic vector sensors: A MUSIC approach. In Noise and Vibration: Emerging Methods, pages 1-10. ISVR, 2009.

[Wyl76] H. W. Wyld. Mathematical methods for physics. W.A. Benjamin, Advanced Book Program, Reading, Mass, 1976.

[YLSC08] T. Yardibi, J. Li, P. Stoica, and L. N. Cattafesta. Sparsity constrained deconvolution approaches for acoustic source mapping. The Journal of the Acoustical Society of America, 123(5):2631-2642, may 2008.

[ZH05] H. Zou and T. Hastie. Regularization and variable selection via the elastic net. Journal of the Royal Statistical Society: Series B (Statistical Methodology), 67(2):301-320, apr 2005. 